Hunt Research Graup Un Uniwersity of Missauri

\title{
PARAXIAL APPLICATION OF AUXILIARY DEVICES WITH WAVEGUIDE MEDIATED LASER IRRADIATION FOR APPLICATIONS IN MEDICAL BIOPHOTONICS
}

\author{
A Dissertation presented to \\ the Faculty of the Graduate School \\ at the University of Missouri \\ In Partial Fulfillment \\ of the Requirements for the Degree \\ Doctor of Philosophy \\ by \\ PAUL JAMES DOUGLAS WHITESIDE \\ Heather K. Hunt, Ph.D. \\ Dissertation Supervisor \\ May 2017
}




\section{Declaration of Approval}

The undersigned, appointed by the Dean of the Graduate School, have examined the dissertation entitled:

PARAXIAL APPLICATION OF AUXILIARY DEVICES WITH WAVEGUIDE MEDIATED LASER IRRADIATION FOR APPLICATIONS IN MEDICAL BIOPHOTONICS

presented by Paul J.D. Whiteside, a candidate for the degree of Doctor of Philosophy in Bioengineering, and hereby certify that, in their opinion, it is worthy of acceptance.

Heather K. Hunt, Ph.D.

Sheila A. Grant, Ph.D.

Kevin D. Gillis, Ph.D.

Nicholas J. Golda, M.D.

John A. Viator, Ph.D. 
Dedicated to my parents, Clive and Barbara Whiteside 
"I may not have gone where I intended to go,
but I think I've ended up where I needed to be"

The Long Dark Tea-Time of the Soul (1988)

- Douglas Adams

"No man is an island"

Meditation XVII (1624)

- John Donne 


\section{Acknowledgements}

This research has been funded from a variety of sources, including partial funding from an NIH grant, the A. Ward Ford translational research grant, Wallace H. Coulter Foundation translational research grant, and the MU Fast Track translational research grant.

Throughout my graduate research career, there have been many people who fundamentally contributed to my work either scientifically or morally, without whose unique and invaluable contributions, this dissertation may never have successfully come to fruition; whereas there have been others, whose proclivity for distraction and knack for providing ample sources of extracurricular activities easily lengthened this dissertation by a few years.

I would first like to thank my parents, Clive and Barbara Whiteside, for their resolute and continuous support of my madness, because no one in their right mind could ever justify intentionally choosing to get a Ph.D. Having such constant and unwavering support was invaluable in finishing my continued education.

I would also like to thank Dr. Hunt and Dr. Viator, for giving me the freedom, reassurance, and support to innovate new projects and technologies; and I'd like to really thank Dr. Golda for somehow managing to coin a brilliantly catchy phrase for yet another of my best inventions: Dr. Golda coined both "sonoillumination" and "selective release waveguides."

My projects have also benefitted from the technical and moral support of Dr. Benjamin Goldschmidt, who helped to confirm that my mad ideas weren't entirely insane and might actually work out. Our approach to scientific research is fundamentally different, and for that I could not be more grateful, as it has led to more constructive discourse than I enjoy with anyone else. Additionally, I have received help and support from the likes of Quentin Bostick, Michael Carraher, Mason Schellenberg, Jeff Chininis, Emma Bennett, Amanda Sain, Chenxi Qian, and Sharanya Kumar, in addition to the services of the MU EMC and Mizzou Meat Market.

Finally, I would like to thank Dr. Ashley Ermer, who has been a constant source of support and compassion, and who has complicated my life in all the best ways. 


\section{Contents}

Acknowledgements $\quad$ ii

List of Figures $\quad$ vii

List of Tables $\quad$ ix

Abbreviations $\quad \mathrm{x}$

Biological Terminology xii

Physical Constants xiii

Symbols $\quad$ xiv

$\begin{array}{ll}\text { Abstract } & \text { XV }\end{array}$

1 Introduction to biophotonics and waveguide-mediated irradiation 1

1.1 Applications of lasers in biophotonics . . . . . . . . . . . . . . . 1

1.1.1 Selective photothermolysis ............. . . . 3

1.1.2 Deep tissue thermal effects . . . . . . . . . . . . . . 5

1.2 Laser-tissue interactions . . . . . . . . . . . . . . . . . . 7

1.3 Modern methods in biophotonics . . . . . . . . . . . . . . . 11

1.3.1 Common practices ................. . . 11

1.3.2 Concerns regarding contemporary systems . . . . . . . . . . 13

1.3.2.1 Problems . . . . . . . . . . . . . . . 14

1.3.2.2 Limitations . . . . . . . . . . . . . . . 16

1.4 Contact-based transmission modality . . . . . . . . . . . . 18

1.4.1 Prior Investigations . . . . . . . . . . . . . . . 19

1.4.1.1 Preliminary Methods . . . . . . . . . . . . . . 21

1.4.1.2 Preliminary Results . . . . . . . . . . . . . 23

1.4.2 Branching projects encompassed by this dissertation . . . . . 25 
2 Techniques and challenges of metal thin film characterization for $\begin{array}{ll}\text { applications in photonics } & 28\end{array}$

2.1 Introduction to metal films . . . . . . . . . . . . . . 28

2.1.1 Importance of film thickness in optics . . . . . . . . . . . 30

2.1.2 Metal thin film characterization . . . . . . . . . . . . 33

2.2 Established characterization techniques . . . . . . . . . . . . 35

2.2 .1 X-Ray Reflectivity . . . . . . . . . . . . . . 37

2.2.1.1 XRR: Theory . . . . . . . . . . . . . 37

2.2.1.2 XRR: Method . . . . . . . . . . . . . . 40

2.2.1.3 XRR: Analysis . . . . . . . . . . . . . . 42

2.2 .2 Ellipsometry . . . . . . . . . . . . . . . . . . . . . 44

2.2.2.1 Ellipsometry: Theory _. . . . . . . . . . . . 44

2.2.2.2 Ellipsometry: Method . . . . . . . . . . . . 46

2.2.2.3 Ellipsometry: Analysis . . . . . . . . . . . . . . . 48

2.2 .3 Atomic Force Microscopy . . . . . . . . . . . . . . . . . 51

2.2.3.1 AFM: Theory . . . . . . . . . . . . . . 51

2.2.3.2 AFM: Method . . . . . . . . . . . . 54

2.2.3.3 AFM: Analysis . . . . . . . . . . . . . 55

2.2 .4 Electron Beam Techniques . . . . . . . . . . . . . . . 57

2.2.4.1 E-Beam Techniques: Theory . . . . . . . . . . . 58

2.2.4.2 E-Beam Techniques: Method ............ 61

2.2.4.3 E-Beam Techniques: Cross-Sectional SEM Analysis . 63

2.2.4.4 E-Beam Techniques: SEM-EDS Analysis . . . . . . . 66

2.3 Characterization limitations \& considerations . . . . . . . . . 68

2.3 .1 Radiative absorption . . . . . . . . . . . . . . . . . 68

2.3 .2 Sample conductivity . . . . . . . . . . . . . . . . . . 69

2.3.3 Derived vs. measured thickness . . . . . . . . . . . . . 71

2.4 Conclusions and developing technologies . . . . . . . . . . 72

3 Selective release waveguides and contact-based laser irradiation of tissue $\quad 76$

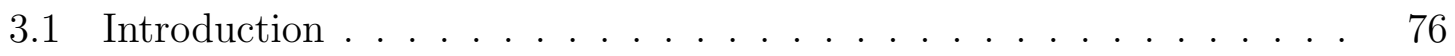

3.2 Technical discussion . . . . . . . . . . . . . . . . . . . . . 77

3.2.1 Operating conditions of planar optical waveguides . . . . 78

3.2.1.1 Maxwell's and Fresnel's Equations . . . . . . . . . 78

3.2 .1 .2 Snell's Law . . . . . . . . . . . . . . . . 86

3.2.1.3 Waveguide operation . . . . . . . . . . . . . 89

3.2.2 Evanescent fields and optical tunneling . . . . . . . . . . . 93

3.2.2.1 Evanescent fields . . . . . . . . . . . . . . . 94

3.2.2.2 Frustrated total internal reflection . . . . . . . . 99

3.2 .3 Contact-based transmission _. . . . . . . . . . . . . . 104

3.2 .4 Objectives . . . . . . . . . . . . . . 106 
3.3 Materials and methods . . . . . . . . . . . . . . . . . . 108

3.3.1 Porcine tissue samples . . . . . . . . . . . . . . . . . 108

3.3.2 Waveguide fabrication . . . . . . . . . . . . . . . . . 109

3.3.3 Experimental apparatus . . . . . . . . . . . . . . . . . 111

3.3.4 Experimental methodology . . . . . . . . . . . . . 113

3.4 Results . . . . . . . . . . . . . . . . . . . 113

3.5 Discussion . . . . . . . . . . . . . . . . . . 117

4 Simultaneous paraxial ultrasonic pulsation to improve transdermal laser fluence $\quad 119$

4.1 Introduction . . . . . . . . . . . . . . . . . . . . 119

4.1.1 Objectives . . . . . . . . . . . . . . . . . 124

4.2 Phenomenological model . . . . . . . . . . . . . . . . 126

4.2.1 Established acousto-optic theory . . . . . . . . . . . . 126

4.2.2 Established UOT theory . . . . . . . . . . . . . . . . . . . 130

4.2.3 Mechanisms of sonoillumination . . . . . . . . . . . 131

4.2.3.1 Refractive index modulation . . . . . . . . . . . . . 132

4.2.3.2 Alteration of optical collision events . . . . . . . . . 134

4.3 Materials and methods . . . . . . . . . . . . . . . . . 139

4.3.1 Porcine tissue samples . . . . . . . . . . . . . . . . . 139

4.3.2 Waveguide fabrication . . . . . . . . . . . . . . . . . . 140

4.3.3 Experimental apparatus . . . . . . . . . . . . . . . 142

4.3.4 Ultrasonic Pulsation . . . . . . . . . . . . . . . . . . . 145

4.3.5 Experimental procedures . . . . . . . . . . . . . . . 147

4.4 Results . . . . . . . . . . . . . . . . . . . . . . . . 148

4.4.1 Electrical Impedance Measurements . . . . . . . . . . . . . . . 148

4.4.2 Relationship between photocurrent and laser energy . . . . . . 153

4.4.3 Optical transmission enhancement . . . . . . . . . . . . . . . . 155

4.5 Discussion . . . . . . . . . . . . . . . . . . . . . . . 158

4.6 Conclusions and continued research . . . . . . . . . . . . . . 164

5 Backward-mode waveguide-mediated photoacoustic tomography 166

5.1 Introduction . . . . . . . . . . . . . . . . . 166

5.2 Technical discussion . . . . . . . . . . . . . . . . . . . . . . . . . . 169

5.2 .1 Photoacoustics . . . . . . . . . . . . . . . . . . 170

5.2.2 Backward-mode transmission modality . . . . . . . . . . 171

5.2 .3 Technical objectives . . . . . . . . . . . . . . . . . . . . 174

5.3 Materials and methods . . . . . . . . . . . . . . . . . 175

5.3 .1 Porcine tissue preparation . . . . . . . . . . . 175

5.3.2 Turbid tissue phantom design . . . . . . . . . . . . 176

5.3 .3 Waveguide fabrication . . . . . . . . . . . . . 177

5.3.4 Experimental apparatus . . . . . . . . . . . . . . . . . 179 
5.3.4.1 Optical apparatus .............. . . 179

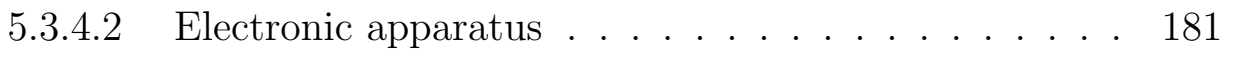

5.3.4.3 Multiplexer design . . . . . . . . . . . . . . . . . 182

5.4 Results. . . . . . . . . . . . . . . . . . . . 188

5.4.1 Preliminary results . . . . . . . . . . . . . . . . . . 189

5.4 2D Depth Profiling . . . . . . . . . . . . . . 190

5.5 Discussion . . . . . . . . . . . . . . . . . . . . . 191

6 Conclusions \& continued investigations 195

6.1 Conclusions . . . . . . . . . . . . . . . . 195

6.2 Continued investigations . . . . . . . . . . . . . . . . . . 197

6.2.1 Project: Selective-release waveguides . . . . . . . . . . . 197

6.2.2 Project: Sonoillumination . . . . . . . . . . . . . . . 199

6.2.3 Project: Waveguide-mediated PAT . . . . . . . . . . . . 201

A Derivation of Theoretical Equations 203

A.1 Maxwell's Equations . . . . . . . . . . . . . . . . . 203

A.1.1 Wave Equation in terms of $\boldsymbol{E}$. . . . . . . . . . . . . . . 203

A.1.2 Wave Equation in terms of $\boldsymbol{H}$. . . . . . . . . . . . . 207

A.1.3 Characteristic Optical Admittance, $\mathcal{Y}$. . . . . . . . . . . . . . 208

A.1.4 The Poynting Vector, Irradiance, and the Optical Absorption Coefficient . . . . . . . . . . . . . . . 211

A.1.5 Snell's Law . . . . . . . . . . . . . . . . . . . 213

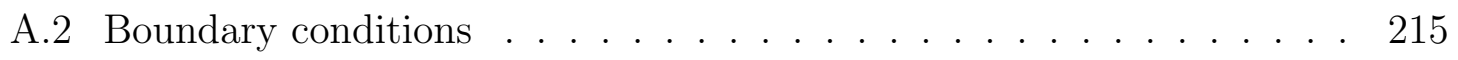

A.2.1 Normal incidence in non-absorbing media . . . . . . . . . . . 215

A.2.2 Oblique incidence in non-absorbing media . . . . . . . . . . 218

A.2.2.1 p-polarized light (TM polarized) . . . . . . . . . . 218

A.2.2.2 s-polarized light (TE polarized) . . . . . . . . . 223

A.2.2.3 Optical admittance for oblique incidence . . . . . . . 227

A.2.2.4 The Brewster Angle . . . . . . . . . . . . . . 229

B MATLAB Simulations $\quad 231$

C Error Analysis of Labview-acquired data 235

C.1 Identifying sources of error . . . . . . . . . . . . . . . 236

C.2 Error compensation . . . . . . . . . . . . . . . 240

$\begin{array}{lr}\text { Bibliography } & 249\end{array}$

$\begin{array}{ll}\text { Vita } & 281\end{array}$ 


\section{List of Figures}

1.1 Graph of Laser-Tissue Interactions . . . . . . . . . . . . . . 3

1.2 Laser tattoo removal example: before and after . . . . . . . . 4

1.3 Diagram of fractional photothermolysis . . . . . . . . . . 6

1.4 Photothermal effects within biological tissue . . . . . . . . . 8

1.5 Cynosure MedLite $C^{6}$ hand-piece . . . . . . . . . . . . . . . 12

1.6 Original theoretical operation of contact-based transmission waveguides 20

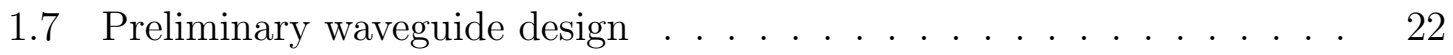

1.8 Preliminary apparatus utilized in M.S. Thesis work . . . . . . . 23

1.9 Preliminary angular spectra of waveguides used in M.S. Thesis . . . . 24

2.1 Metal films utilized on planar waveguides . . . . . . . . . . . 31

2.2 Importance of thickness for evanescent effects . . . . . . . . . . . 33

2.3 XRR theoretical diagram . . . . . . . . . . . . . . . . . . . . 39

2.4 XRR equipment diagram . . . . . . . . . . . . . . . . . . 41

2.5 XRR sample data analysis . . . . . . . . . . . . . . . . . . . 42

2.6 Spectroscopic Ellipsometry equipment diagram . . . . . . . . . . 47

2.7 Spectroscopic Ellipsometry sample data analysis . . . . . . . . . . . . 49

2.8 AFM theoretical diagram . . . . . . . . . . . . . . . . . . 53

2.9 AFM equipment diagram . . . . . . . . . . . . . . . . . . . 54

2.10 AFM sample data analysis . . . . . . . . . . . . 56

2.11 E-beam interaction volume . . . . . . . . . . . . . . . . 59

2.12 SEM equipment diagram . . . . . . . . . . . . . . . 62

2.13 SEM sample data analysis . . . . . . . . . . . . . . 64

2.14 EDS sample data analysis $\ldots \ldots \ldots \ldots \ldots \ldots$

3.1 Oblique incidence sign conventions for TM and TE polarizations . . . 81

3.2 Fresnel Conditions . . . . . . . . . . . . . . . . . . . . 87

3.3 Reflectivity vs. Internal Reflection Angle: Glass-Air . . . . . . . . . . 91

3.4 Diagram of light propagation within a waveguide . . . . . . . . 92

3.5 Reflectivity vs. Internal Reflection Angle: Glass-Silver . . . . . . . . 93

3.6 Evanescent field penetration depth: Glass-Air . . . . . . . . . . 97

3.7 Evanescent field penetration depth $\ldots \ldots \ldots \ldots$ 
3.8 Diagram of Evanescent Penetration . . . . . . . . . . . . . . 100

3.9 Diagram of Optical Tunneling . . . . . . . . . . . . . . . . . . . . . 101

3.10 Boundary conditions for FTIR . . . . . . . . . . . . . . . . . . . . . 102

3.11 Application of SRW in laser dermatology . . . . . . . . . . . 106

3.12 SRW physical design . . . . . . . . . . . . . . . . . . . . . . 109

3.13 Optical thin film regulation experimental apparatus . . . . . . . . . 112

3.14 SEM-EDS analysis spectrum for Ag on Si substrate . . . . . . . . . . 114

3.15 Results of preliminary waveguide transmission experiments . . . . . 116

3.16 Optical transmission through tissue vs. film thickness . . . . . . . . 117

4.1 Graph of chromophore spectral absorption . . . . . . . . . . . . . . 121

4.2 Sonoillumination modulating tissue . . . . . . . . . . . . . 125

4.3 Sonoillumination theoretical diagram . . . . . . . . . . . . . . . 131

4.4 Porcine tissue sample cartridge . . . . . . . . . . . . . . . 140

4.5 Waveguide design for Sonoillumination applications . . . . . . . . 141

4.6 Experimental apparatus for Sonoillumination . . . . . . . . . . . 142

4.7 3D printed optomechanical mount for Sonoillumination . . . . . . . . 144

4.8 Equivalent L-match circuit design . . . . . . . . . . . . . . 146

4.9 Resistance and reactance of the $500 \mathrm{kHz}$ transducer . . . . . . . . . 152

4.10 Graph of photocurrent vs. laser energy . . . . . . . . . . . . . 154

4.11 Graph of transmitted optical power by driving voltage . . . . . . 156

4.12 Sonoillumination improvement factor vs. driving voltage . . . . . 157

4.13 Resistance and reactance of an impedance matched transducer . . . . 159

4.14 Comparison of impedance magnitudes between transducer and matched circuit . . . . . . . . . . . . . . . . . . . . . 161

5.1 Waveguide-mediated photoacoustics . . . . . . . . . . . . . 172

5.2 Preliminary PAT waveguide design . . . . . . . . . . . . . . . 177

5.3 Optical apparatus of photoacoustic system . . . . . . . . . . 179

5.4 3D printed photoacoustic tomography cartridge . . . . . . . . 180

5.5 Imasonic array transducer design $\ldots \ldots \ldots \ldots . \ldots \ldots$

5.6 Multiplexer PCB design . . . . . . . . . . . . . . . . . . . . 185

5.7 Photograph of the Multiplexer PCB . . . . . . . . . . . . 186

5.8 Photograph of the completed multiplexer . . . . . . . . . . . . . 188

5.9 Photoacoustic traces . . . . . . . . . . . . . . . . . . . . 190

6.1 Waveguides with multi-layer thin film claddings . . . . . . . . . 198

6.2 Acoustic wedge for pulsing into tissue at an angle . . . . . . . . 200

C.1 Error from pulse to pulse energy variation . . . . . . . . . . 239 


\section{List of Tables}

2.1 Examples of metal films in optics and biophotonics . . . . . . 30

2.2 List of metal film characterization techniques . . . . . . . . . 36

3.1 Tabulated atomic ratios and thicknesses for Ag and Ti films . . . . 115

4.1 Theorized threshold frequencies for sonoillumination efficacy . . . . 139

4.2 Tabulated transducer complex electrical impedance values . . . . . . 152

4.3 Photocurrent regression statistics . . . . . . . . . . . 155

4.4 Tabulated amplification statistics by driving amplitude . . . . . . 163

5.1 Tabulated electronic components used for MUX boards . . . . . . 187

5.2 Calculated speed of sound in tissue phantoms . . . . . . . . . 192 


\title{
Abbreviations
}

\author{
AFM Atomic Force Microscopy \\ ATR Attenuated Total Reflection \\ CW Continuous Wave \\ DAQ Data Acquisition \\ EMC Electron Microscopy Core \\ Er:YAG Erbrium doped Yttrium Aluminum Garnet \\ FS $\quad$ Fused Silica \\ FTIR Frustrated Total Internal Reflection \\ GRIF Gradient Refractive Index Field \\ GRIN Graded Index \\ LASER Light Amplification by Stimulated Emission of Radiation \\ LASIK Laser-Assisted In Situ Keratomileusis \\ MRI Magnetic Resonance Imaging \\ MTZ MicroThermal Zone \\ N-BK7 Borosilicate Crown Glass \\ N-IR Near-Infrared \\ Nd:YAG Neodymium-doped Yttrium Aluminum Garnet \\ NOA 74 Norland Optical Adhesive \# 74 \\ OCA Optical Clearing Agent \\ OCT Optical Coherence Tomography \\ PAQT Photonic Ablation via Quantum Tunneling \\ PAS PhotoAcoustic Spectroscopy
}


PAT PhotoAcoustic Tomography

PC PolyCarbonate

PEG Polyethylene Glycol

PMMA PolyMethylMethAcrylate (i.e. Acrylic)

PXI PCI extensions for Instrumentation

SEM Scanning Electron Microscopy

SRW Selective Release Waveguide

TE Transverse Electronic

TIR Total Internal Reflection

TIRPAS Total Internal Reflection PhotoAcoustic Spectroscopy

TIZ Targeted Irradiation Zone

TM Transverse Magnetic

TOC Tissue Optical Clearing

TOCD Tissue Optical Clearing Device

UOT Ultrasound-modulated Optical Tomography

UV Ultraviolet 


\title{
Biological Terminology
}

\author{
Collagen \\ Structural protein giving skin its elasticity \\ Dermis \\ Epidermis \\ Lower layer of skin containing blood vessels and hair follicles \\ Fibroblasts \\ Outer layer of skin containing melanin \\ Fibroplasia \\ Collagen producing cells \\ Hyperpigmentation \\ Formation of fibrous tissue to create the extracellular matrix \\ Hyperthermia \\ Dramatic relative increase in local melanin content \\ Hypopigmentation \\ Elevated body temperature due to failed thermoregulation \\ Hypertrophic scar \\ Dramatic relative decrease in local melanin content \\ Inflammation \\ Raised scars that protrude and distort tissue topography \\ Lymphatic system \\ The body's response to harmful stimuli and damage \\ Melanin \\ Vessels that transport constituents of the inflammatory response \\ Melasma \\ Broadly optically absorbing pigment in skin and hair \\ Necrosis \\ Brown or grey-brown patches of discoloration in skin \\ Oedema \\ Cell death that releases cell contents into extracellular matrix \\ Phagocytosis \\ Area of fluid retention causing tissue swelling \\ Port wine stain \\ Process by which a cell engulfs a solid particle \\ Purpura \\ Reddish area of capillary malformation in the skin \\ Thermal coagulation \\ Vascular system \\ Red or purple discolorations in the skin \\ Vascularization \\ Denaturation of blood cells through intense heat \\ White blood cell \\ System of vessels and capillaries through which blood flows \\ Formation of blood vessels and capillaries in living tissue \\ Cells responsible for engulfing and removing foreign elements
}




\section{Physical Constants}

Speed of Light in a vacuum

Speed of Sound in water

Speed of Sound in tissue

Speed of sound in acrylic

Speed of sound in fused silica

Acoustic impedance of water

Acoustic impedance of tissue

Acoustic impedance of acrylic

Acoustic impedance of fused silica

Refractive index of silver $(532 \mathrm{~nm})$

Refractive index of titanium $(532 \mathrm{~nm})$

Refractive index of acrylic (532 nm)

Refractive index of fused silica $(532 \mathrm{~nm})$

Extinction coefficient of silver $(532 \mathrm{~nm})$

Extinction coefficient of titanium $(532 \mathrm{~nm})$

Permittivity of free space

Permeability of free space

$$
\begin{aligned}
c & =2.9979 \times 10^{8} \mathrm{~m} \mathrm{~s}^{-1} \\
v_{\text {water }} & =1.497 \times 10^{3} \mathrm{~m} \mathrm{~s}^{-1} \\
v_{\text {tissue }} & =1.470 \times 10^{3} \mathrm{~m} \mathrm{~s}^{-1} \\
v_{\text {acrylic }} & =2.750 \times 10^{3} \mathrm{~m} \mathrm{~s}^{-1} \\
v_{F S} & =5.960 \times 10^{3} \mathrm{~m} \mathrm{~s}^{-1} \\
Z_{\text {water }} & =1.483 \times 10^{5} \mathrm{~g} / \mathrm{cm}^{2} \mathrm{~s}^{-1} \\
Z_{\text {tissue }} & =1.580 \times 10^{5} \mathrm{~g} / \mathrm{cm}^{2} \mathrm{~s}^{-1} \\
Z_{\text {acrylic }} & =3.260 \times 10^{5} \mathrm{~g} / \mathrm{cm}^{2} \mathrm{~s}^{-1} \\
Z_{f s} & =13.1 \times 10^{5} \mathrm{~g} / \mathrm{cm}^{2} \mathrm{~s}^{-1} \\
n_{A g} & =0.1429 \\
n_{\text {Ti }} & =2.479 \\
n_{\text {acrylic }} & =1.4953 \\
n_{F S} & =1.4607 \\
k_{A g} & =3.0518 \\
k_{T i} & =3.3511 \\
\epsilon_{0} & =8.8541 \times 10^{-12} \mathrm{~m}^{-3} \mathrm{~kg}^{-1} \mathrm{~s}^{4} \mathrm{~A}^{2} \\
\mu_{0} & =1.2566 \times 10^{-6} \mathrm{~m} \mathrm{~kg} \mathrm{~s}^{-2} \mathrm{~A}^{-2}
\end{aligned}
$$




\section{Symbols}

\begin{tabular}{|c|c|c|}
\hline$N$ & complex refractive index & (unitless) \\
\hline$n$ & real component of $N$ & (unitless) \\
\hline$k$ & complex component of $N$ & (unitless) \\
\hline$\lambda$ & wavelength & $\mathrm{nm}$ \\
\hline$P$ & power & $\mathrm{mW}\left(\mathrm{mJ} \mathrm{s}^{-1}\right)$ \\
\hline$I$ & intensity & $\mathrm{mJ}$ \\
\hline $\mathbf{H}$ & Magnetic field strength & $\mathrm{A} \mathrm{m}^{-1}$ \\
\hline$B$ & Magnetic flux density & $\mathrm{T}$ \\
\hline $\mathrm{E}$ & Electric field strength & $\mathrm{V} \mathrm{m} \mathrm{m}^{-1}$ \\
\hline$D$ & Electric displacement & $\mathrm{V} \mathrm{m}^{-2}$ \\
\hline$j$ & Electric current density & $\mathrm{A} \mathrm{m} \mathrm{m}^{-2}$ \\
\hline$\rho$ & Electric charge density & $\mathrm{C} \mathrm{m} \mathrm{m}^{-3}$ \\
\hline$\sigma$ & Electric conductivity & $\mathrm{S} \mathrm{m}^{-1}$ \\
\hline$\mu$ & permeability & $\mathrm{m} \mathrm{kg} \mathrm{s}^{-2} \mathrm{~A}^{-2}$ \\
\hline$\epsilon$ & permittivity & $\mathrm{m}^{-3} \mathrm{~kg}^{-1} \mathrm{~s}^{4} \mathrm{~A}^{2}$ \\
\hline$\mu_{r}$ & relative permeability & $\mathrm{m} \mathrm{kg} \mathrm{s}^{-2} \mathrm{~A}^{-2}$ \\
\hline$\epsilon_{r}$ & relative permeability & $\mathrm{m}^{-3} \mathrm{~kg}^{-1} \mathrm{~s}^{4} \mathrm{~A}^{2}$ \\
\hline$T$ & Relative transmission coefficient & (unitless) \\
\hline$R$ & Relative reflection coefficient & (unitless) \\
\hline$\Gamma_{g}$ & Gruneissen coefficient & (unitless) \\
\hline$H_{B}$ & Radiant beam exposure & $\mathrm{J} \mathrm{cm}^{-2}$ \\
\hline$Z$ & Electrical Impedance & $\Omega$ \\
\hline
\end{tabular}


UNIVERSITY OF MISSOURI

\title{
Paraxial application of auxiliary devices with waveguide mediated laser irradiation for applications in medical biophotonics
}

\author{
Paul James Douglas Whiteside
}

Heather K. Hunt, Dissertation Supervisor

\section{Abstract}

Laser-based medical applications offer minimally-invasive alternatives to traditional procedures; however, the simplistic method of open-air laser transmission poses ocular hazards and negative side effects, along with a fundamentally limited efficacy for patients of darker complexion due to strong optical absorption in the epidermis. Additionally, the traditional irradiation method also inhibits the incorporation of additional technologies that might otherwise enhance therapeutic effects or provide diagnostic benefits, as doing so would otherwise occlude the laser beam path. The research presented herein addresses each of these considerations individually, first by transmitting laser light into tissue through direct contact with a selective-release waveguide, and thereafter incorporating auxiliary equipment on its rear face. Metal clad planar optical waveguides are demonstrated for the transmission of laser light into samples of porcine skin through direct transmission, governed by scaling evanescent leaking through a designated active area by controlling thin film thickness. In one manifestation, an ultrasonic pulser was incorporated to modulate tissue optical properties and thereby improve transmission of light through epidermal and dermal tissues by increasing forward anisotropy; whereas in another, a high frequency ultrasonic transducer was incorporated to detect photoacoustically generated pressure waves to determine depth profiles of chromophores in skin as a foundation for clinical backward-mode photoacoustic tomography. 


\section{Chapter 1}

\section{Introduction to biophotonics and waveguide-mediated irradiation}

Portions of this chapter have been reproduced with permission from the Master's

Thesis work entitled Photonic ablation via quantum tunneling, authored by Paul J.D. Whiteside, Bioengineering Department, University of Missouri, May 2015 [1].

\subsection{Applications of lasers in biophotonics}

Although initially not intended for such applications, lasers have been incorporated into a wide variety of medical procedures, demonstrating strong appeal as a highly controllable, non-invasive instrument. In particular, dermatology has been among the most predominant fields with applications of lasers in medicine, including tattoo removal, port wine stain treatment, skin resurfacing, treatment of acne vulgaris, and ablation of vascular lesions and cancerous tumors [2-4]. However, while many of the procedures are similar in nature, each of these applications requires the use of a different variety of laser based primarily on the intended biological or chemical target to be illuminated [5].

That being said, laser procedures in general can be broken down into five distinct 
classifications, depending on the characteristics of the laser being utilized and the duration of the laser pulse: photodisruption, plasma-induced ablation, photoablation, thermal interaction, and photochemical interaction $[6,7]$. The first three methods utilize pulse durations in the range of femto- to nano-seconds, as shown in Figure 1.1, whereas the latter two employ durations in the millisecond to continuous wave $(\mathrm{CW})$ range. It is worth noting that although the shorter pulse durations do not individually exhibit thermal effects identical to those produced using longer pulses, similar results have been observed when the repetition rate of the laser and number of pulses have been sufficiently high [8]. Consequently, in certain cases, short-pulse duration approaches may be used for long-pulse duration applications; e.g. utilizing short-pulse fractionated $\mathrm{CO}_{2}$ laser ablation for skin resurfacing.

However, while there are a plethora of applications for lasers in clinical dermatology, the method of choice for any given procedure generally reduces to one of two principally desired effects - either the localized ablation of a target chromophore, or the incitement of large area photothermal effects within the tissue. Principal examples of the former effect include the treatment of port wine stains, solar lentigines (lesions), and pigmented tumors, along with the removal of percutaneous tattoos and other foreign pigments [9-11]. By contrast, the latter effect typically encompasses broad deep-tissue applications, such as acne scar treatment, skin resurfacing, and hair removal [12-15]. 


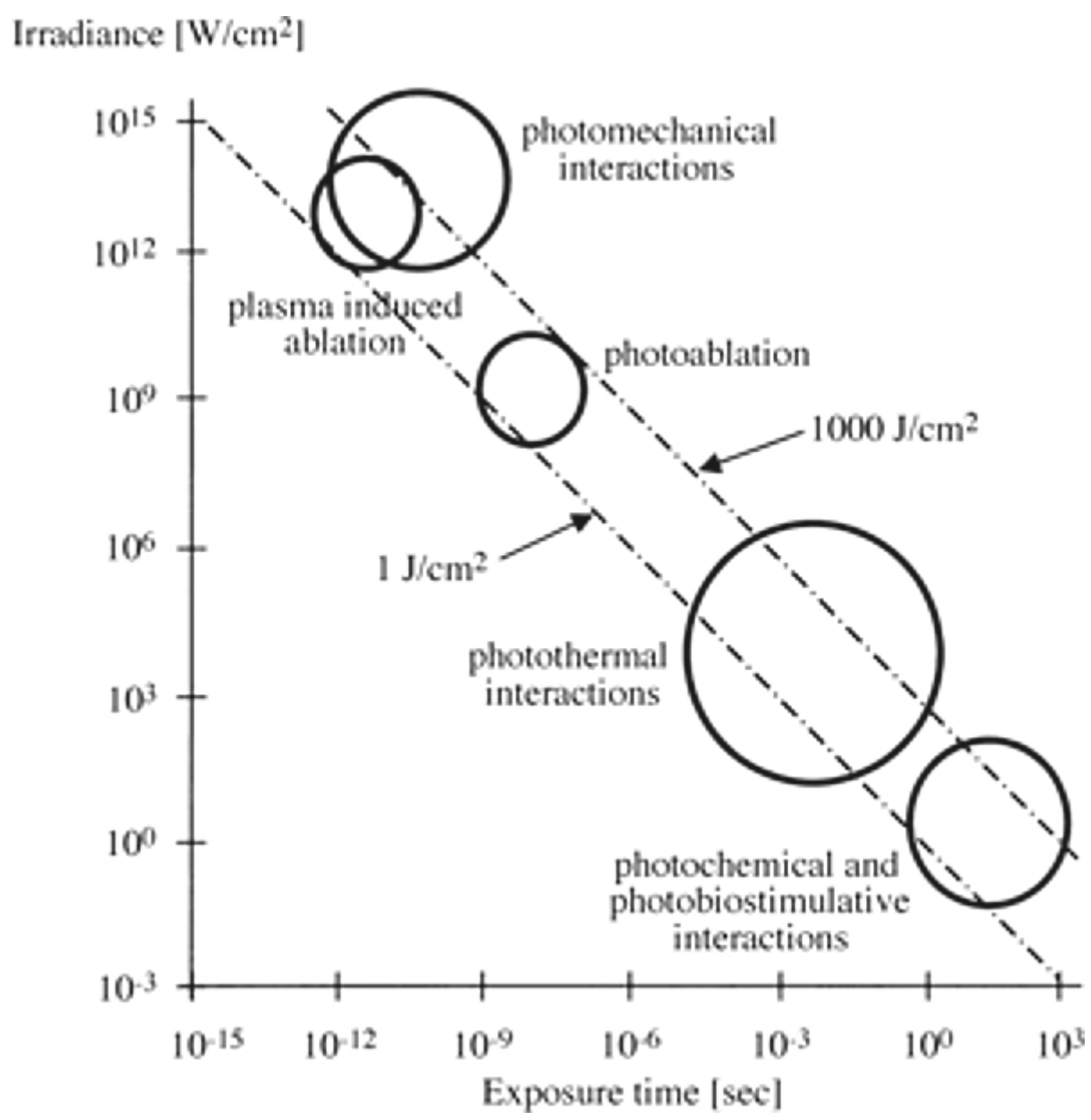

FiguRE 1.1 A depiction of Laser-Tissue interactions as a function of pulse duration and laser power, with the two lines indicating the 1 and $1000 \mathrm{~J} / \mathrm{cm}^{2}$ irradiance limits [6].

\subsubsection{Selective photothermolysis}

A primary example of the first effect, and one of the more predominant applications of lasers in dermatology, is laser tattoo removal. The technique operates on the principle of "selective photothermolysis," in which specific wavelengths of light are chosen to photoablate molecules of tattoo ink, depending upon their relative optical absorption [11]. For example, red and yellow inks tend to clear best when ablated by laser light with a wavelength of $532 \mathrm{~nm}$; whereas green, blue, and black inks tend to 


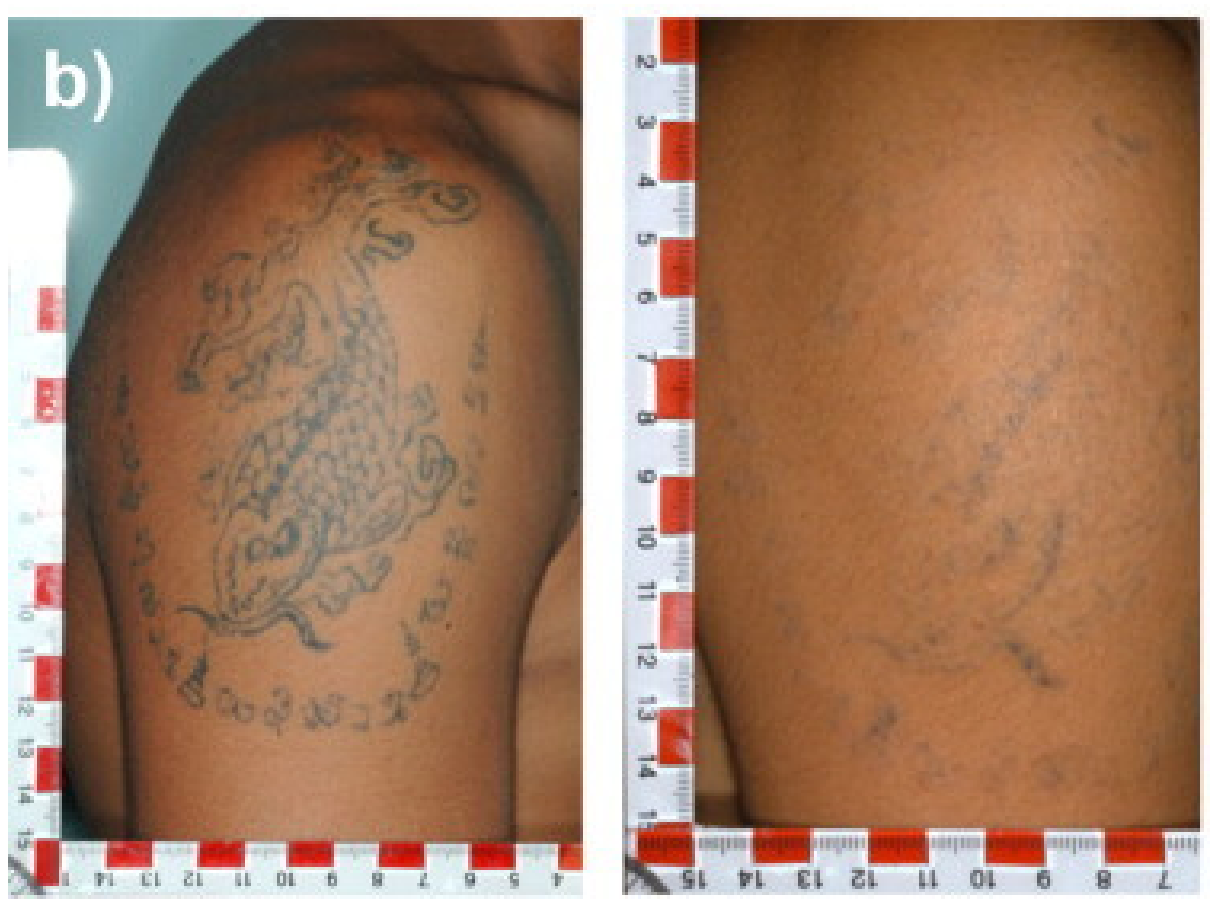

FIGURE 1.2 [left] Monochromatic tattoo prior to laser treatment. [right] The same tattoo after four treatments of $1064 \mathrm{~nm}$ laser light from a Q-Switched Nd:YAG laser with 6 ns pulse duration [18].

clear best using $755 \mathrm{~nm}$ or $1064 \mathrm{~nm}[8,16]$.

Unfortunately, there are currently no restrictions as to the chemical composition of any tattoo inks, their pharmacological consistency, or their purity, the result of which is that each pigment may necessitate a different wavelength based on the optical absorption spectrum of the target ink, which may vary from patient to patient [17]. Additionally, the photoablation may also have adverse medical effects post-operation due to the release of potentially harmful chemicals that previously comprised the ink. For example, certain red inks tend to contain mercury or cadmium that may be released into the body when the ink is ablated, which can result in a substantial immunological response at the site of the tattoo or throughout the body [8].

Figure 1.2 demonstrates a comparison of the results of a series of four laser tat- 
too removal treatments using a Q-switched Nd:YAG operating at $1064 \mathrm{~nm}$. Although individual treatments tend not to completely clear the tattoos, they are often preferable to more invasive surgical procedures, which necessitate complex wound closing procedures, potentially resulting in wound healing complications, hypertrophic scarring, keloid formation, or anatomic distortion; consequently, laser tattoo removal procedures generally consist of a lengthy series of treatments (between 4 - 20 treatments depending on the complexity and size of the tattoo) [19-21]. Nevertheless, laser tattoo removal has shown wider appeal and greater success than other treatment procedures that tend to scar or injure the surrounding tissue, with methods being refined and improved through medical laser research every year [22].

\subsubsection{Deep tissue thermal effects}

Another major application of lasers in dermatology is in the field of skin resurfacing. Whereas tattoo removal procedures operate through photothermolysis, skin resurfacing techniques capitalize on broader, deep-tissue photothermal effects in order to achieve results. Laser skin resurfacing has fairly broad applications from acne scar removal to skin tightening and wrinkle removal, but the general effect is the same regardless of the structure to be resurfaced [23].

By utilizing relatively long pulse-widths (millisecond regime), the technique focuses on the thermal interactions that occur in the area surrounding the illuminated portion [15]. The high-energy laser light carbonizes and photocoagulates surface tissue, causing necrosis in the top-most layer of the epidermis and an inflammatory response in the deeper dermal tissue. The consequence of this photothermal damage 

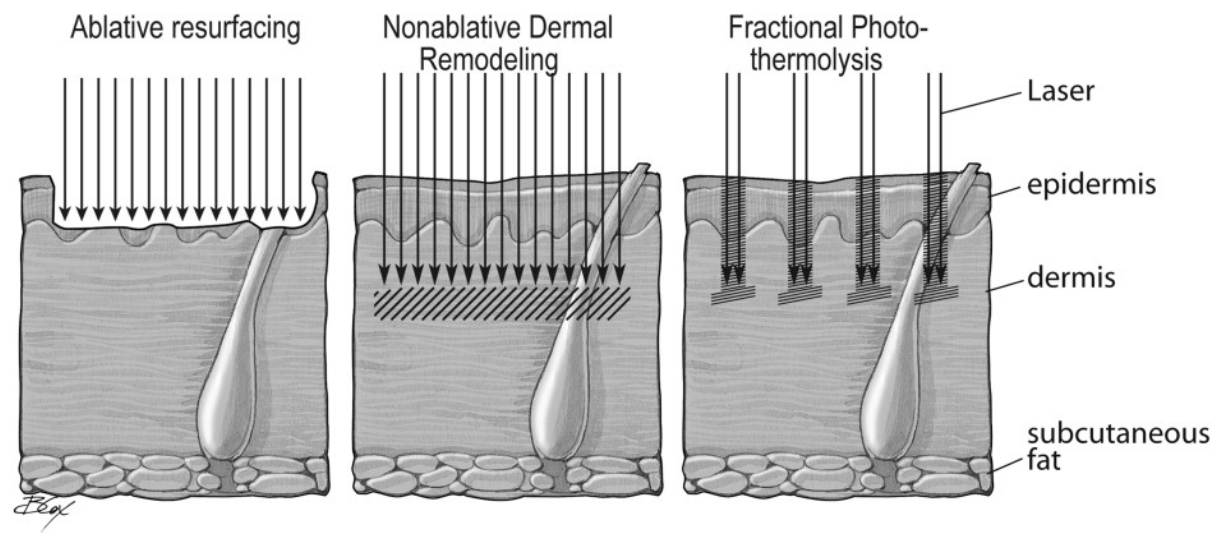

FigURE 1.3 Fractional photothermolysis ablates columns of tissue to encourage collagen production in the dermis, without causing excess damage to the epidermis as in ablative resurfacing techniques [27].

is that the scarred surface tissue is destroyed and subsequently removed, whereas the deeper dermal tissue experiences an increase in collagen production to promote the generation of well-structured, healthy tissue [24].

Unlike techniques that target specific pigmented chromophores, skin resurfacing techniques target dissolved water molecules within the tissue; consequently, the lasers used tend to operate in the IR or near-IR wavelength spectra, since water is strongly optically absorbing in that range. The lasers typically used in skin resurfacing tend to be $\mathrm{CO}_{2}$, Er:YAG, or Nd:YAG lasers with pulse durations from microseconds to continuous wave $[13,25,26]$.

Additionally, the techniques for laser skin resurfacing can be divided into three categories - ablative resurfacing, nonablative dermal remodeling, and fractional photothermolysis - with each method focusing on a different aspect of the photothermal response [27, 28]. Ablative resurfacing is primarily intended to evenly destroy surface tissue, as shown in Figure 1.3 [left], and is useful for treating hypertrophic scarring $[24,29]$. Nonablative dermal remodeling, by contrast, focuses on homogeneously en- 
couraging the increased production of collagen without causing excess surface tissue damage, as shown in Figure 1.3 [center], and is useful for wrinkle removal procedures [30].

Fractional photothermolysis, however, relies on the heterogeneous ablation of tissue, leaving portions of healthy tissue interspersed between treated regions, as shown in Figure 1.3 [right], and is used in the treatment of pigmented lesions, melasma, and acne scarring $[27,31]$. The intention of this approach is to cause partial photothermolysis in the treated regions, referred to as "microthermal zones" (MTZs), so as to achieve similar, albeit lessened, resurfacing effects as in ablative resurfacing, while leaving the surrounding tissue to experience similar inflammatory wound healing responses to those observed in nonablative dermal remodeling $[24,32]$. Research has shown that Fractionated methods may result in faster recovery times by reserving healthy tissue to more readily repair the laser-damaged tissue due to keratinocyte diffusion from the untreated into the treated zones [4]. Additionally, the non-ablative thermal damage to the surrounding tissue tightens and organizes collagen fibers while simultaneously promoting neocollagen secretion by fibroblasts $[33,34]$. However, non-Fractionated methods are still widely used in treatments based on manufacturers' preferences and the relative youth of the Fractionated methods in clinical applications.

\subsection{Laser-tissue interactions}

When examining the effects of laser light on biological tissue, there are a myriad of considerations that may dramatically influence the final approach from an engineer- 


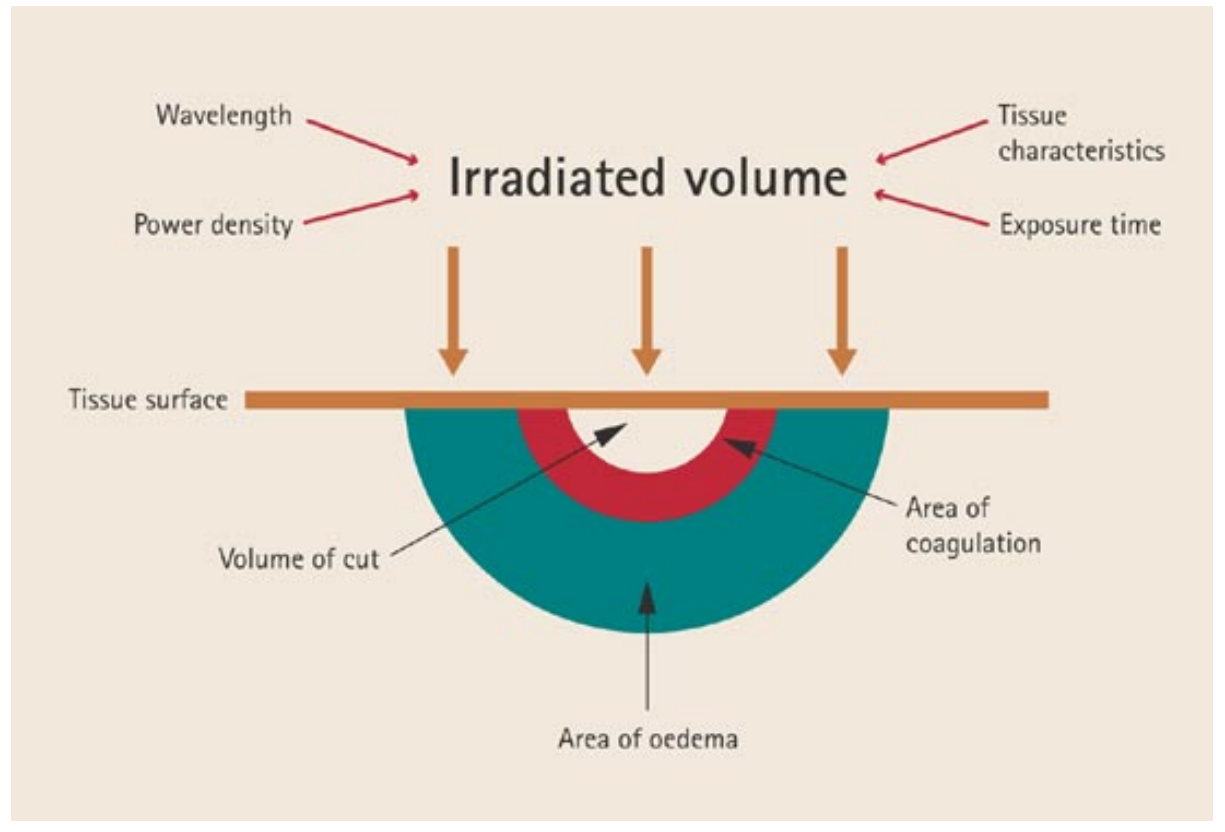

FIGURE 1.4 The severity of the thermal effects observed following illumination of biological soft tissue with incident high-energy laser light decreases with penetration depth into the tissue [35].

ing perspective. Specific examples of such factors include inhomogenous scattering and absorption within the tissue, the tissue's density and water content, and the degree of reflection at the surface of the tissue [35]. However, for dermatological applications it is convenient to consider only the case of soft epithelial tissues like skin. Whereas the specific effects will vary from person to person based on aspects like melanin, fat, and water content, the resultant thermal effects within the tissue remain largely consistent from person to person.

Regardless of the application, illuminating soft tissues with a high-powered laser will result in a layering of distinct photothermal effects, resultant from the tissue's poor heat conductivity into the atmosphere coupled with its inherent thermal relaxation time $[35,36]$. These effects differ between applications based on the laser's pulsewidth, energy density, and wavelength, along with the tissue's own optical prop- 
erties; however, as shown in Figure 1.4, the order of their occurrence by depth remains largely consistent: vaporization, carbonization, coagulation, oedema, and hyperthermia.

The topmost phenomenon involves the vaporzation of intracellular water within the skin. At temperatures in excess of $100^{\circ} \mathrm{C}$, water molecules within the surface tissue vaporize, which induces ruptures and thermal decomposition resulting in localized tissue cavitation [6]. This cavitation is then reinforced by the subsequent layer of carbonization, which is a consequence of insufficient thermal conduction of excess heat away from the tissue surface. When utilizing the laser as an optical scalpel, the carbonization is useful in sustaining the cut by preventing the tissue from reforming; however, for most dermatology applications it is considered an undesirable consequence of laser illumination.

The remaining layers of thermal coagulation, oedema, and hyperthermia are considered to be more reversible than previous two phenomena, since the body has mechanisms for their repair. These layers elicit both immunological and would-healing responses within the tissue, which have been shown to result in increased collagen production and reduced vascularization, the principal goals of skin resurfacing and port-wine stain treatments respectively $[7,37]$. However, when considering the photoablation of individual pigmented chromophores - like tattoo inks, contrast agents, or pigmented lesions - the aforementioned photothermal effects are typically considered to be concomitant localized tissue reactions, rather than intentional biological responses. In such cases, the primary goal of the procedure is the photo-induced destruction of the target chromophore through a combination of either photothermolysis 
or photoplasmolysis along with inelastic photoacoustic expansion (photomechanical action), based on the pulse width and energy density of the laser [6].

Photothermolysis, as discussed in Section 1.1.1, is intended to break apart the target chromophore into much smaller molecules by thermally compromising their structural integrity. The effect is achieved by using ultra-short pulse durations in the nanosecond regime to induce an acoustic shockwave, whose intensity exceeds the fracture thresholds of the molecules [38]. The acoustic pressure wave is produced through the rapid thermal expansion of the chromophore immediately following optical absorption, in a process referred to as "photoacoustics." Due to the extreme energies of the incident beam, the photoacoustic expansion is inelastic, fragmenting the target into smaller particles, which are more easily phagocytosed - engulfed by white blood cells - for removal by the vascular or lymphatic systems [39]. Additionally, the fragmentation of the chromophore may generate particles whose dimensions are smaller than the wavelength of visible light, and would therefore be effectively "cleared" regardless of their presence or absence in the tissue.

Photoplasmolysis acts in a similar fashion and produces a photoacoustic pressure wave through the same inelastic expansion; however, the principal difference between photoplasmolysis and photothermolysis is that the former effect results in the optical breakdown and subsequent plasma formation by the target chromophore formed by the ionizing effects of the incident beam [40-42]. Consequently, the ensuing explosive inelastic expansion is much more extreme and results in a more thorough destruction of the pigment [35]. The two effects are distinguished by the pulse width of the incident beam, with photoplasmolysis exclusively occurring with pulse widths shorter 
than 1000 ps and photothermolysis occupying the longer pulse width regime up to a few hundred nanoseconds.

\subsection{Modern methods in biophotonics}

Regardless of the desired clinical effect, however, the means of tissue irradiation in clinical practice is largely consistent across devices, with only slight differences based on the intended application or the manufacturer's proprietary designs. Although the method is simple and straight-forward, it exposes the patient and practitioner to significant hazards, while also being fundamentally limited in that it necessitates a clear line of sight to the target, which precludes the incorporation of auxiliary technologies.

\subsubsection{Common practices}

This method of laser transmission, which has been systematically accepted throughout the clinical community, operates through the free-space open-air transmission of light aimed in the direction of the target tissue [43]. The techniques typically involve routing the light into a tethered hand-piece by means of either a fiber-optic bundle or an articulated mirror system, depending on the energy requirements of the application. An adjustable lens within the hand-piece focuses the light onto the tissue surface while the operating distance is set by an adjustable or interchangeable stand-off or plastic guard, such as the thin metal stand-off attached to the end of the hand-piece shown in Figure 1.5.

In addition to directing the laser light toward the tissue, medical laser man- 


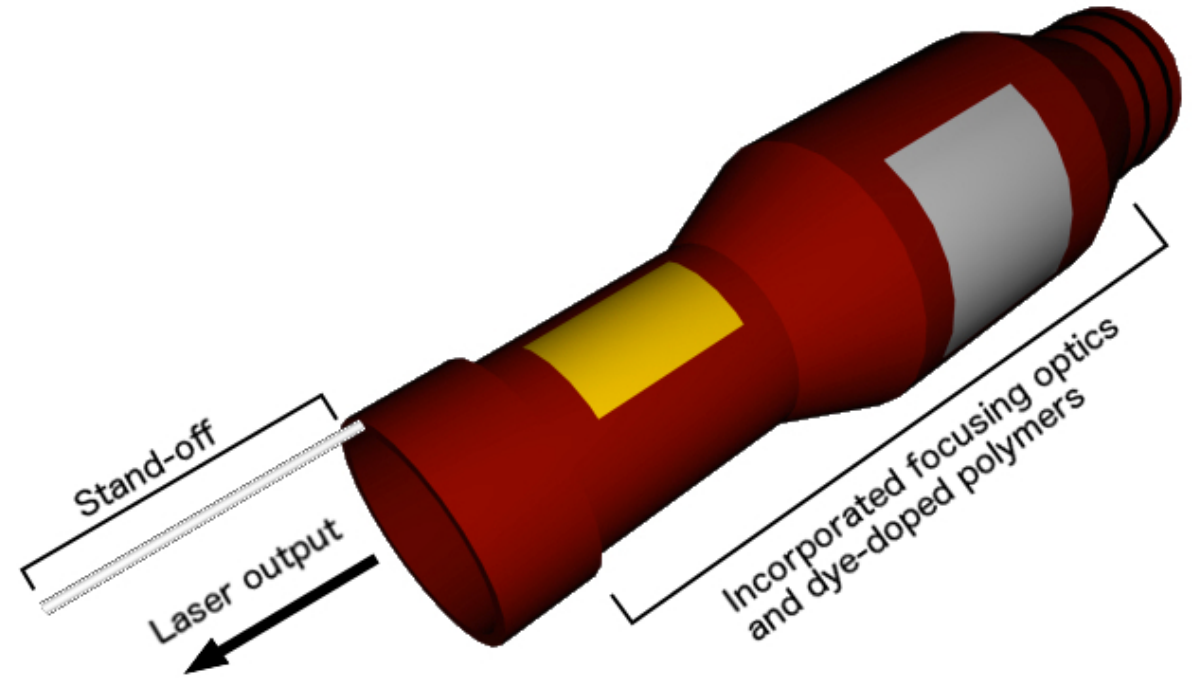

Figure 1.5 The Cynosure MedLite $C^{6}$ laser system utilizes interchangeable hand-pieces containing dye-doped modified polymers that increase the frequency of the Nd:YAG output from $532 \mathrm{~nm}$ to either 585 or $650 \mathrm{~nm}$ for applications in selective photothermolysis [44, 45].

ufacturers may incorporate interchangeable hand-pieces to enhance the capabilities of their systems. For example, a company called Cynosure currently produces the MedLite $C^{6}$ laser tattoo removal system, which is comprised of a powerful Q-Switched Nd:YAG laser that is directed toward a hand-piece via an articulated mirror system [45]. The Nd:YAG's principal harmonic output is a beam of $1064 \mathrm{~nm}$ IR light; the beam can also be frequency doubled to output the secondary harmonic beam at $532 \mathrm{~nm}$ in the visible spectrum. However, the MedLite $C^{6}$ system, also incorporates interchangeable hand-pieces, as shown in Figure 1.5, which contain dye-doped modified polymers. These polymers are pumped by the frequency doubled Nd:YAG in order to output lower frequency beams. For example, a polymer within the hand-piece doped with Pyrromethene-650 (PM-650) dye will output a beam at $650 \mathrm{~nm}$, whereas Pyrromethene-597 (PM-597) will output a beam at $585 \mathrm{~nm}$ [44]. Consequently, such devices are primarily utilized in the selective photothermolysis of multi-colored tat- 
toos for applications wherein the Nd:YAG's harmonic outputs are insufficient (i.e. for the ablation of chromophores that are not strongly absorbing at either 532 or $1064 \mathrm{~nm})$.

Other companies produce similar units, each of which illuminates the tissue through free-space delivery of light out of a hand-piece. The principal difference between any two systems typically simplifies down to the lasing material (Nd:YAG, Er:YAG, $\mathrm{CO}_{2}$, etc.), the pulse width, and the manufacturer's proprietary technologies, like the aforementioned dye-doped polymers. However, certain manufacturers also incorporate a means of fractionating the beam out of the hand-piece for fractional photothermolysis applications, as discussed in Section 1.1.2. For example, the Fraxel Laser System, produced by Solta Medical, employs a $\mathrm{CO}_{2}$ laser that is directed into a hand-piece wherein the beam is split up into an array of smaller beams to create thousands of microthermal zones (MTZs) [46].

\subsubsection{Concerns regarding contemporary systems}

Although laser procedures have progressed substantially since the technology was first put into practice by Leon Goldman in 1963, modern techniques still exhibit many of the same detrimental characteristics as their forebears $[9,47,48]$. These characteristics manifest as both distinctly detrimental operational hazards and fundamental limiting factors that compromise the efficacy of light-based medical procedures. With regard to operational hazards, two of the more predominant problems involve the risk of ocular injury to the practitioner and the potential for negative tissue effects within the targeted tissue [49-52]. These concerns are directly resultant 
from the operational design of the light delivery systems used in dermatology settings. Moreover, all such designs regardless of application are subject to the same limitation, in that they necessitate a clear line-of-sight to the optical target. This requirement inhibits the incorporation of auxiliary devices, barring significant alteration to the secondary technology, as the vast majority would otherwise occlude the path of the light.

\subsubsection{Problems}

With regard to contemporary problems, the open-air, free-space delivery method involves a distinct and consistent possibility for the un-confined light to reflect off the patient's skin. Considering that the vast majority of techniques utilize laser energies between 1 and $1000 \mathrm{~J} / \mathrm{cm}^{2}$, as per Figure 1.1, a diffuse reflection of even just 1\% of the incident energy may be enough to cause permanent eye damage to anyone present [49]. This is particularly the case for ultra-short pulsed laser systems in the picosecond to microsecond regimes, as the pulse can result in microcavitation or photoacoustic destruction within retinal melanosomes, in addition to intra-retinal damage produced through the ionizing effects of picosecond pulse lasers [53]. Moreover, it has been reported that the vast majority of laser related injuries involve serious damage to the retina, which consideration is only made worse by the extremely limited medical or surgical treatments available to treat ocular laser injuries [49].

Consequently, in all dermatological laser applications, the practitioner and the patient are required to wear protective eyewear with a high optical density for the wavelength being used [54]. The high optical density reduces the degree to which 
those wavelengths transmit into the retina; however, most eyewear occludes a broad spectrum of wavelengths, rather than a single specific wavelength [55]. While these glasses serve to protect the wearer from light-based injury, the high optical density also dramatically hinders the vision of the laser operator across a broad spectrum of wavelengths. Clinical practitioners therefore develop procedural habits wherein they intermittently remove the eyewear between laser pulses so that they can visually identify the target more clearly. This practice is currently a procedural necessity, due to their limited vision; however, it entirely undermines the purpose of the protective glasses in the event of a mistake.

Another consequence of the free-space propagation design is that the light is transmitted into the skin at a tissue-air interface. As discussed in Section 1.2, the poor heat conductivity at this interface prevents adequate heat dissipation, resulting in carbonization, vaporization, and a subsequent inflammatory response. Whereas most of these effects fade within a few days of treatment, more permanent hyperpigmentation, hypopigmentation, purpura, and scarring are possible side effects of excessive damage or uneven wound healing [56]. Moreover, the potential for damage increases noticeably for patients of darker skin phototypes (i.e. higher melanin content or density), so much greater care must be taken for such patients $[57,58]$.

There are also other factors related to the delivery design that further exacerbate these problems. For example, there is a substantial degree of operator error introduced because the hand-pieces are aimed at transdermal targets held suspended above the tissue surface, which can result in harmful overexposure of the tissue, causing permanent scarring and increased recovery times. Moreover, due to the in- 
consistencies of the laser delivery methods and the rapid rate at which the oedema forms within the tissue, most procedures necessitate a lengthy series of treatments, rather than a single, more effective treatment, which only serves to compound the aforementioned concerns with every subsequent visit, particularly in pigment clearing procedures like tattoo removal [19, 21, 25].

\subsubsection{Limitations}

With regard to the incorporation of auxiliary devices, in order to utilize laser transmission alongside any secondary technology, modern transmission modalities demand that modifications be made to the secondary technique, which can often compromise the effectiveness or otherwise require more complex alternatives to achieve the same effect. One such example that has seen limited implementation in clinical laser dermatology is the contact cooling of tissue during laser ablation procedures. In the course of laser treatment, a significant amount of heat is generated through the undesired optical absorption of the incident beam by biological chromophores within the tissue (e.g. melanin, hemoglobin, water) [36]. This heating can permanently damage tissue through carbonization and coagulation and is the primary source of pain during procedures due to the thermal damage caused to local sensory nerves [6, 35]. Contact cooling techniques have been suggested as a possible means of either mitigating this thermal damage or otherwise cooling the tissue prior to the procedure to numb nerve tissue and reduce patient pain [19]. However, contemporary methods of transmitting laser light necessitate that cooling devices must not occlude the path of the beam. This requirement has thus far effectively limited the viable options for cooling the 
tissue during procedures to the use of cold air or cryogenic sprays, as cooling pads would block the incident laser light [2, 28, 30, 59].

Another example of an application that attempts to incorporate laser irradiation alongside additional equipment is in the field of photoacoustic tomography, which involves the generation of ultrasonic pressure waves within tissue following the absorption of a sufficiently short pulse of light [60-62]. Due to factors such as acoustic attenuation and impedance mismatches between soft and hard tissues, applications of photoacoustics in a clinical setting necessitate that the acoustic detectors record signals from the same side of the tissue from which they were generated, a condition referred to as "backward mode." This requirement has proven challenging to perform outside of a laboratory setting, since traditional ultrasonic transducers in this orientation either occlude the path of the laser beam or otherwise must be positioned off-axis. Nevertheless, as with the tissue cooling technologies, alternative approaches have been developed that modify the secondary detection equipment in order to allow for a freely propagating laser beam. One such solution, presented by Zhang et al. resolved the occlusion issue by utilizing a dual-wavelength optical method of ultrasonic measurement [63]. The system utilizes two lasers of different wavelengths superimposed on each other incident upon a Fabry-Pérot (F-P) etalon manufactured with dichroic films surrounding a compressible transparent polymer layer [64]. This arrangement allows one of the beams to excite the photoacoustic response within the tissue, while the other is used to record the interference response of the F-P cavity, which varies as the thickness of the polymer layer is changed when the ultrasonic pressure wave hits its surface. Similar F-P cavities have also been minimized to the 
tip of a $125 \mu \mathrm{m}$ optical fiber acting as an optical hydrophone, which can be positioned in an array around a central illuminating optical fiber for photoacoustic generation [65].

\subsection{Contact-based transmission modality}

Based on these examples, the standard approach to resolving the occlusion problem observed in dual-technology systems typically involves modifying the secondary technology in order to accommodate a freely propagating laser beam, which may place additional design constraints or limitations on their use. Meanwhile, the standard approach to resolving the ocular hazards presented by reflections involves the use of occluding eyewear, which presents issues in and of itself. These concerns gave rise to the contact-based transmission modality around which this dissertation is focused. The method, originally referred to as photonic ablation via quantum tunneling (PAQT), departs from traditional free-space propagation in favor of a thin planar optical waveguide that delivers the light via direct physical contact with the tissue. This method encloses the optical system, which circumvents hazards associated with backscattered light and prevents occlusion of the light while allowing for the paraxial operation of a second technology on the rear face of the waveguide [66]. For example, in the case of photoacoustic tomography, a traditional piezoelectric ultrasonic transducer or array may be positioned on the rear face of the waveguide to detect photoacoustic waves propagating out from the tissue, through the waveguide substrate, toward the detector surface [67]. The modality was originally investigated in my Master's Thesis and forms the foundation for the various applications inves- 
tigated in this Dissertation. The following sections provide a brief summary of the prior research, which established the core principles of the technology.

\subsubsection{Prior Investigations}

The following sections cover the preliminary findings in the foundation of the contact-based method as presented in the manuscript entitled Regulation of evanescent leaking for contact-based light transmission in biophotonics applications, authored by Paul J.D. Whiteside, Nicholas J. Golda, Randy D. Curry, and John A. Viator, which has been submitted for publication in the journal Applied Optics [68].

For the purposes of this project, the PAQT waveguides consisted of glass slabs clad in layers of titanium and silver. The waveguides also incorporated rectangular "active areas" consisting of thin films of those same metals, which allow the light to optically tunnel into tissue phantoms by means of evanescent leaking. The degree of optical tunneling is strongly related to the incident angle of the light and the thickness of the film in the active area, so waveguides were scanned throughout a range of internal reflection angles using cylindrical coupling lenses, as shown in Figure 1.6, and transmitted through films of four different thicknesses. The specific objectives of this study were to:

- establish waveguide fabrication protocols;

- record a wide range of internal reflection geometries using hemi-cylindrical coupling lenses;

- demonstrate laser beam transmission into agar gel tissue phantoms; 


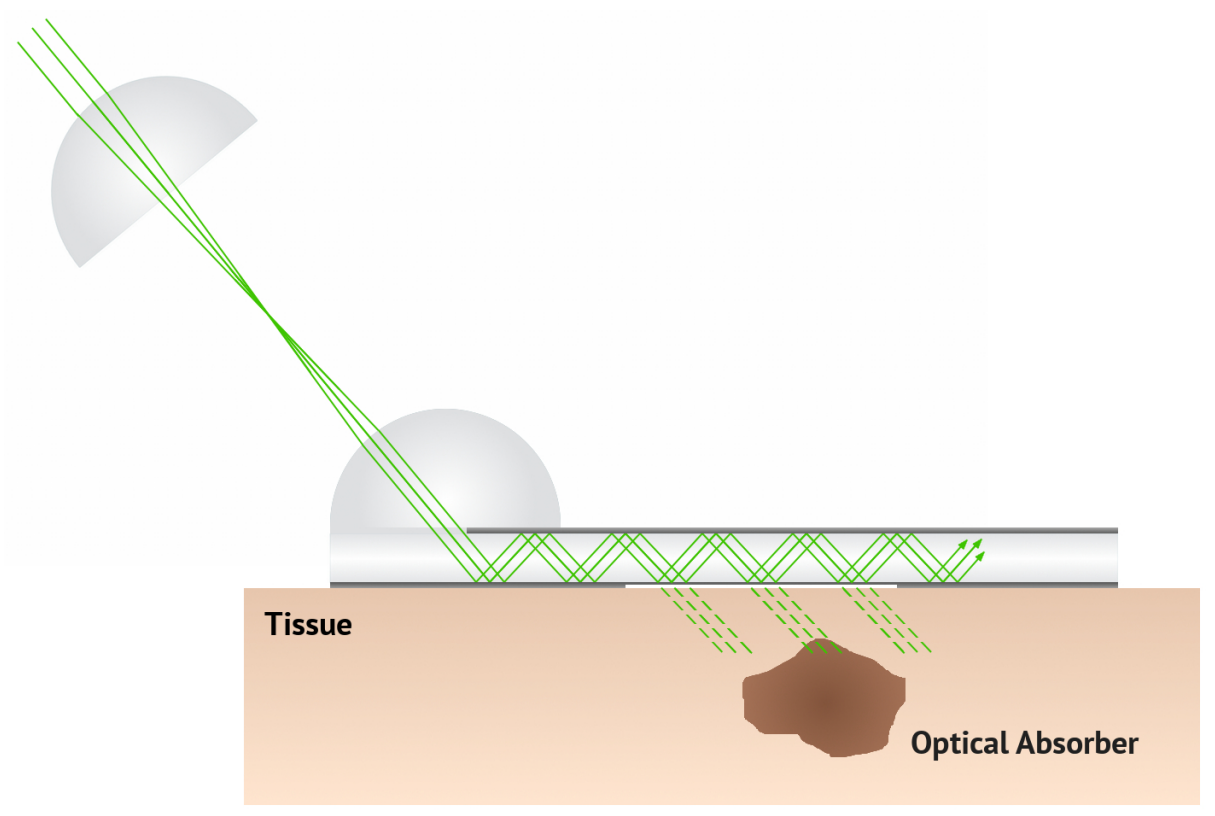

FiguRE 1.6 Coupling light directly into tissue by physical contact circumvents many of the issues presented by free-space propagation techniques, and the use of cylindrical coupling lenses permits tuning of the internal reflection angles.

- regulate transmission efficiency by varying active area film thickness.

The modality presented herein is heavily dependent upon both macroscale optical waveguide transmission along with the sub-wavelength evanescent effects demonstrated by photons undergoing total internal reflection, which concepts will be discussed more thoroughly in Chapter 3. Optical waveguides are a well established technology and have been used extensively in a variety of applications, including optoelectronic integrated circuitry, attenuated total reflection spectroscopy (ATR), and laser beam generation within planar waveguides [69-71]. Light is transmitted along the length of the substrate by internally reflecting between their bounding surfaces, as a result of refractive index differences between substrate and cladding media. Although most waveguides utilize polymer claddings $\left(n_{2} \geq 1.32\right)$ to ensure total inter- 
nal reflection (TIR) conditions and permit waveguide operation, the objectives of this study necessitated an uncommonly low critical angle, which could not be achieved with any commercially available polymer. Consequently, the waveguides fabricated for this project consisted of BK7 glass substrates $\left(n_{1}=1.519\right.$ at $\left.532 \mathrm{~nm}\right)$ coated in a layer of silver $\left(n_{2}=0.1429\right)$ applied using a cold sputter coater, resulting in an extremely low critical angle, $\theta_{c}=5.36^{\circ}$, permitting TIR within the waveguide for all incident angles $6^{\circ}<\theta_{1}<90^{\circ}[72,73]$.

In these experiments, laser light was coupled into a planar optical waveguide, wherein it reflected between opposing faces until reaching a thin film active area. When placed in contact with skin or a tissue phantom, the light at each internal reflection point occurring within the active area either refracted or optically tunneled into the external medium, depending on the active area film thickness.

\subsubsection{Preliminary Methods}

The waveguides developed for this study were fabricated in house, utilizing rectangular BK7 glass slabs $1 \mathrm{~mm}$ thick, clad in thick films of silver and titanium, as shown in Figure 1.7. The former was chosen as a cladding due to its exceptionally low refractive index, $n=0.1429$, at the lasing wavelength of $532 \mathrm{~nm}$ that was produced from the second harmonic generator of a Q-switched Nd:YAG laser; whereas the latter was deposited in an ultra-thin film to serve as an adhesion layer for the silver, since silver

does not strongly adhere to glass [73-75]. The entrance aperture geometry measured $6 \mathrm{~mm}$ from the end of the substrate, which permitted the incorporation of a hemicylindrical lens (Thorlabs, LJ1878L2-A) adhered atop the entrance aperture using 


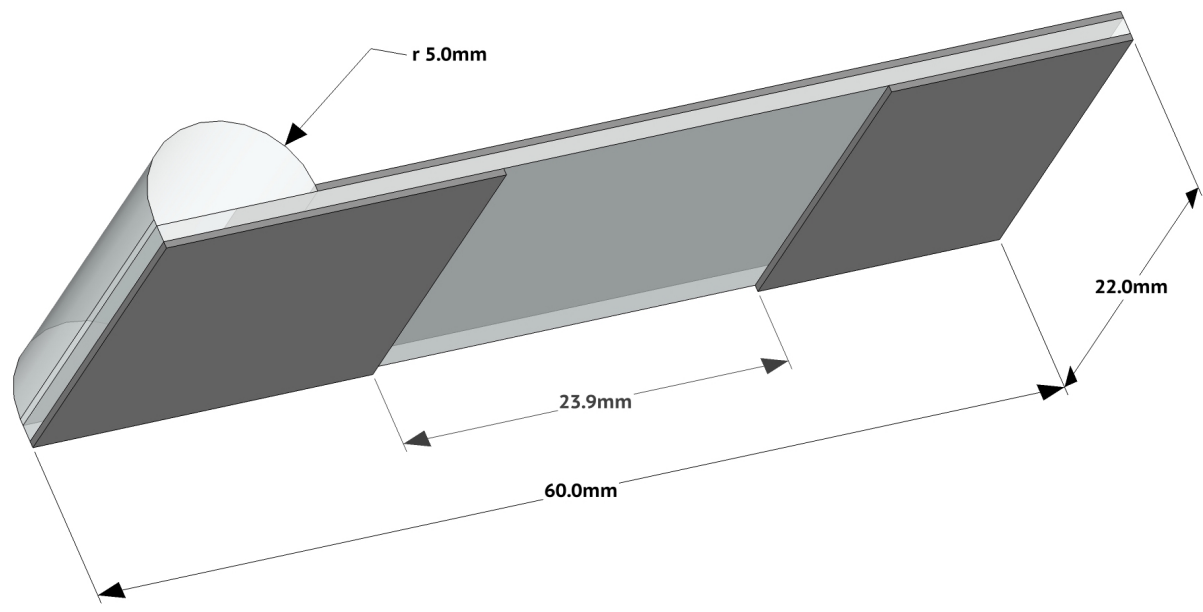

FiguRE 1.7 BK7 glass substrates were clad in thin films of $\mathrm{Ti}$ and $\mathrm{Ag}$ (shown exaggerated above), with a separately deposited active area on the underside of the substrate.

NOA 74 (Norland Optical Adhesive \# 74). A section of the substrate was masked off on the opposite face to serve as the active area, which was designed to begin at approximately $21.10 \mathrm{~mm}$ from the aperture end of the slab and end at approximately $45.0 \mathrm{~mm}$.

Synthetic multi-layered tissue phantoms composed of agar gel and optically absorbing rubber were utilized in these experiments to simulate aspects of human skin. A clear layer of agar separated the waveguide from the rubber absorber, which was intended to represent a target chromophore, such as a blood vessel or cancerous lesion, approximately $3 \mathrm{~mm}$ to $4 \mathrm{~mm}$ below the surface of the tissue. Rubber was chosen as opposed to a dye or other such pigment to prevent the chromophore from diffusing throughout the agar phantom.

A Q-switched Nd:YAG laser (Quantel, Brilliant B), which transmitted a vertically polarized beam at a wavelength of $532 \mathrm{~nm}$ in a circular gaussian profile with a $5 \mathrm{~ns}$ pulse width, was used to generate photoacoustic signals within the tissue phantom by 


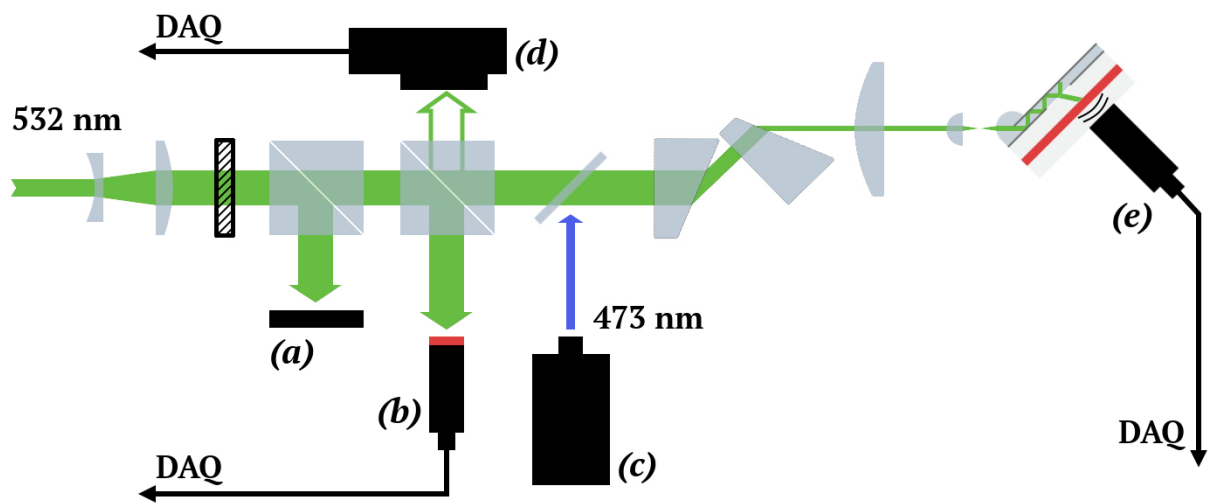

FIGURE 1.8 P-polarized light was directed into a beam dump (a), while s-polarized light transmitted through a 50:50 beamsplitter. Half of the energy directed toward a rubber chromophore adhered to an ultrasonic transducer (b) to measure incident beam energy, while a reflection was used to trigger a photodiode (d). Remaining light was transmitted into the tissue phantom via an optical waveguide to be detected by an ultrasonic transducer (e) for photoacoustic signal analysis.

means of the waveguides developed for this study. Figure 1.8 depicts the optical train used to couple light into the waveguides and demonstrates that the waveguides could be rotated with respect to the incident beam to vary the internal reflection angle. Photoacoustic signals generated within the tissue phantom were recorded throughout the angular spectrum at $0.2^{\circ}$ increments from perpendicular incidence to $75^{\circ}$. Tests were repeated 15 times to improve statistical reliability.

\subsubsection{Preliminary Results}

Figure 1.9 depicts the integrated energy transmission curve throughout the angular spectrum for each of the four active area geometries under consideration. Each of the curves represents the respective mean corrected intensity spectrum calculated from 15 successive angular spectra recorded under identical situations without making any alteration to the apparatus between tests. The angular spectra shown in Figure 1.9 


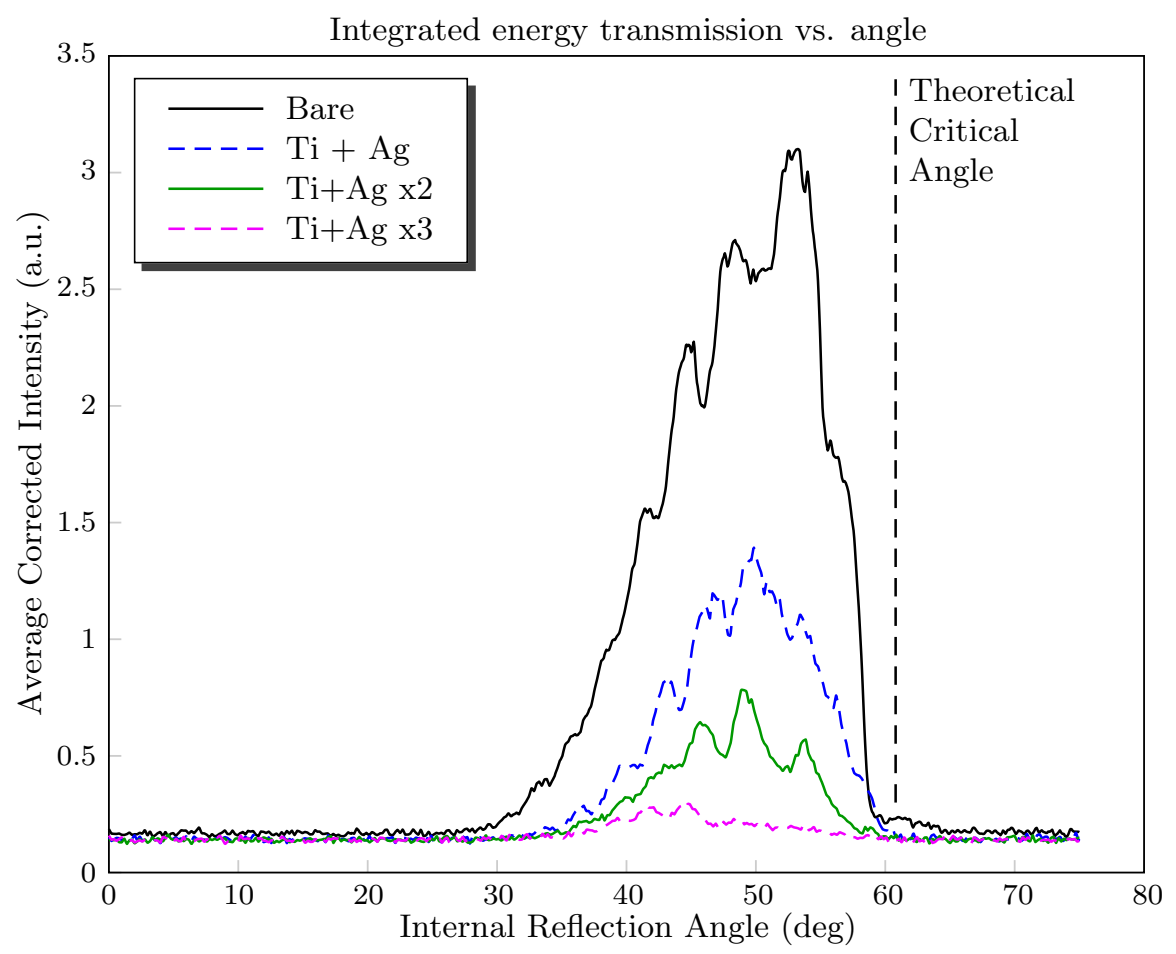

FiguRE 1.9 The decrease in integrated intensity corresponds with the decrease in evanescent penetration into tissue phantoms through silver films with increasing thickness.

demonstrate that the intensity of light transmitted into the phantom reached its maximum near $50^{\circ}$ and decreased with increasing film thickness. The diminishing transmission was expected seeing as the bare active areas transmitted light through the exposed glass substrate by means of simple refraction; whereas the silvered active area geometries acted as partial mirrors, with a portion of the beam transmitting into the phantom by means of optical tunneling. With each subsequent film deposited on top of the previous layers in $30 \mathrm{~nm}$ increments, a smaller portion of the evanescent peak penetrated through the intermediary film layer, which decreased the degree of FTIR that occurred. Accordingly, by specifically controlling the thickness of the thin film in the active area, the angular transmission spectra confirm that the transmitted 
intensity can be intentionally scaled without varying the incident laser intensity, which can be used to design waveguides that transmit different amounts of light at each internal reflection point, depending upon the thickness of the active area film at that location.

These initial experiments demonstrated the potential for transmitting laser light into tissue through direct physical contact. The generation of photoacoustic signals within the tissue demonstrated that the light remained collimated when it reached the chromophore, which indicated that these waveguides were not merely illuminating the tissue with light, but coupling laser beams into the tissue medium through direct physical contact. Furthermore, the results of the silver film active area transmission studies demonstrated that it was also possible to regulate energy transmission at an individual internal reflection location through the control of active area film thickness and composition.

\subsubsection{Branching projects encompassed by this dissertation}

With the proof-of-concept work having successfully established the groundwork for contact-based light transmission, this dissertation represented a collective effort to investigate three potential applications of the waveguide technology, each utilizing aspects of the core technique to achieve unique effects. To more consistently describe the waveguides in such a manner that there would be no confusion between applications, the technology will henceforth be referred to as selective release waveguides (SRWs), owing to the tunability of the active area parameters.

First and foremost, an aspect of this dissertation was dedicated to the logical 
continuation of the original work to more thoroughly characterize the effects observed when using SRWs to transmit light into tissue. This project involved the characterization of sub-micron metal film deposition rates followed by optical transmission testing using ex vivo samples of porcine skin. A variety of metal thin film characterization methods were considered, which led to the compilation of a comprehensive review of the primary techniques used to determine metal film thickness, which has been included as Chapter 2. The principal study, however, investigated ten fused silica waveguides with active area thicknesses ranging from 0 to $60 \mathrm{~nm}$ to establish a greater understanding of the relationship between silver film thickness and light transmission, and has been included as Chapter 3.

The second sub-project built on the back of the SRW technology by incorporating an ultrasonic transducer onto the rear face of an acrylic waveguide. Doing so permitted the ultrasonic modulation of tissue optical properties during laser irradiation of samples of ex vivo porcine skin. The technique combined concepts utilized in acousto-optic modulators with the newly established contact-based laser dermatology method to improve light transmission through epidermal, dermal, and adipose tissue. Results yielded a maximum 1.742 times improvement in optical transmission over the baseline, representing the significant potential for this dual-technology modality, hereafter referred to as sonoillumination, to greatly improve the efficacy of light-based procedures. Results of this study have been incorporated as Chapter 4 of this dissertation.

Finally, Chapter 5 represents the third sub-project, which utilized a 32 element ultrasonic transducer array on the rear face of an acrylic SRW to establish a new 
method of performing 2D backward-mode photoacoustic tomography (PAT). This project involved the design of a 32 channel multiplexer, which was fabricated almost entirely in-house. Whereas most paraxial backward-mode PAT devices modify the acoustic sensing equipment, irradiating the tissue using a planar acrylic SRW permitted the use of a standard transducer array. This project focuses on the potential imaging of severe burn wounds, but is intended to be expanded to investigate pressure ulcers and blood oxygenation to track wound healing and the adhesion of skin grafts. 


\section{Chapter 2}

\section{Techniques and challenges of metal thin film characterization for applications in photonics}

This chapter is reproduced with permission from the manuscript entitled Techniques and Challenges for Characterizing Metal Thin Films with Applications in Photonics, authored by Paul J.D. Whiteside, Jeffrey A. Chininis, and Heather K. Hunt, published as an open-access article in the MDPI journal Coatings [76].

\subsection{Introduction to metal films}

Well before the age of dedicated scientific inquiry and technological innovation, metal-smiths of the ancient world made use of metal thin films to gild less-precious materials in thin layers of gold and silver [77]. Although these craftsmen were likely ignorant of the physiochemical processes involved in their plating procedures, their contributions to metal film deposition techniques have inspired centuries of thin film innovation for applications that extend far beyond their mere artistic origins. Building on that foundation, modern metal thin films are utilized in a variety of scientific and industrial applications, but few more so than in the field of optics. Such films offer 
an uncommon combination of transparency and conductivity for certain compositions and thicknesses, while also boasting broadband reflectivity for relatively thicker films $[78,79]$.

Depending on the desired film characteristics, a variety of different metals have been utilized in thin film optics with applications ranging from optoelectronics to surface plasmon generation, examples of which are provided in Table 2.1 with references for the interested reader. Amongst the more common of these applications, metal films are often deposited in nanoscale thicknesses on ceramic substrates in order to create high-quality broadband mirrored surfaces. Such mirrors have been utilized in reflecting telescopes as alternatives to lens arrays in order to reduce image aberrations, in microscopy for dark-field illumination, or in adaptive optics to create deformable mirrors for optical wavefront control [80-82]. Such high quality mirrors are also widely used in laser applications to exert control over laser beam directionality and propagation. For example, in the field of biophotonics, of particular interest to this work, articulated mirror arms are utilized in clinical laser dermatology systems to redirect high-powered, short-pulsed laser light toward the clinical target; meanwhile, metal film-clad optical waveguides are also being developed for direct-contact coupling of laser light into tissue via optical tunneling, as depicted in Fig. 2.1 [40, 66, 67, 83].

Many contemporary laser systems also make use of metal thin films throughout their optical train, whether involved in the generation of the beam within the lasing medium or the redirection of the beam toward the desired target. For example, in the case of solid lasing media (e.g. Nd:YAG crystals), it is sometimes sufficient to simply polish the opposing ends of the material to an optical grade finish in order 


\begin{tabular}{clll}
\hline \hline Element & Application in optics & Important parameters & Ref. \\
\hline $\mathrm{Al}$ & Microelectronics and optoelectronics & Thickness, conductivity & {$[84]$} \\
$\mathrm{Ti}$ & Adhesion layer for Au \& Ag films & Thickness, adhesion & {$[75]$} \\
$\mathrm{V}$ & Thin film optical switches & Thickness, conductivity & {$[85]$} \\
$\mathrm{Cr}$ & Adhesion layer for Au \& Ag films & Thickness, adhesion & {$[75]$} \\
$\mathrm{Ni}$ & Solar cells and other photovoltaics & Transmittivity, resistivity & {$[86]$} \\
$\mathrm{Cu}$ & Transparent conducting films & Thickness, structure & {$[78]$} \\
$\mathrm{Ag}$ & Thin film coated optical waveguides & Reflectivity, thickness & {$[66]$} \\
$\mathrm{In} / \mathrm{Sn}$ & Solar cells and thin film OLEDs & Transmittivity, resistivity & {$[87]$} \\
$\mathrm{W}$ & Photocatalysis for graphene nanosheets & Optical absorption & {$[88]$} \\
$\mathrm{Au}$ & Surface plasmon generation & Thickness, composition & {$[74]$} \\
\hline \hline
\end{tabular}

TABLE 2.1 Examples of photonics applications employing metal thin films, the majority of whose most important characteristics are either directly dependent upon or can be indirectly related to the film's thickness.

to achieve the lasing action; however, for higher power applications like medical laser ablation, the ends of the medium are often also coated with a highly reflective metal film in order to generate a beam of consistent intensity [89]. In such cases, the facets of the material will be polished to an extreme degree of flatness and parallelism prior to being coated with a thin layer of metal - typically aluminum or silver. In order to allow the beam to escape confinement within the lasing medium, however, one of the metal film mirrors must be partially transmissive.

\subsubsection{Importance of film thickness in optics}

The performance of these mirrors in their respective applications is largely dependent upon three principal factors: the wavelength of light being used, the optical properties of the metal film, and the film's thickness. Of these three, however, the wavelength of light is typically both fixed and known, particularly for laser-related applications. Additionally, the optical properties of standard metals - including absorption, reflectivity, and refractive index - have been extensively researched and are 


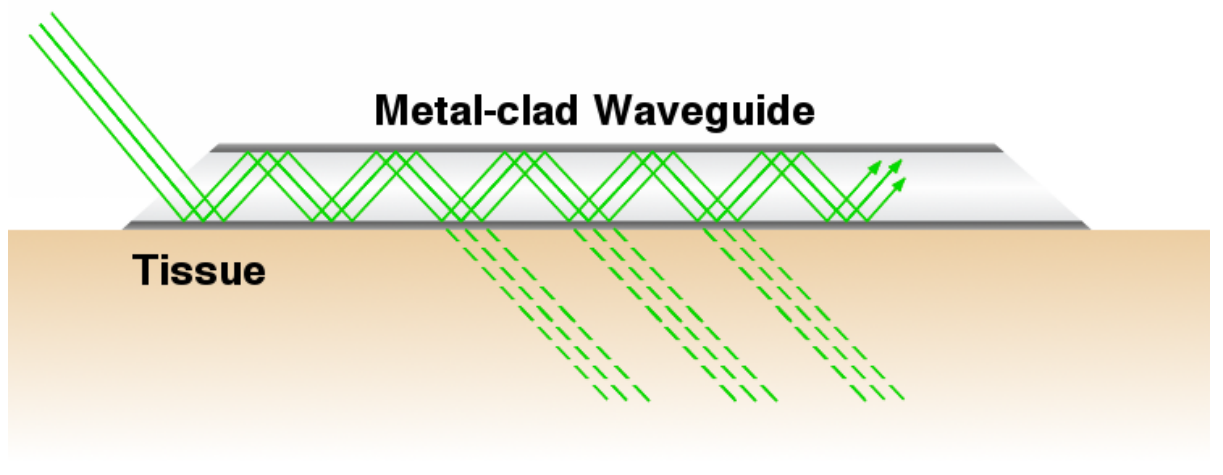

FIgURE 2.1 Regarding the example of a metal coated optical waveguide, the metal thin film serves as a cladding layer to restrict the laser light within the bounds of the substrate; however, upon contact with the tissue, depending on the thickness of the metal film, the light may transmit out of the waveguide [83].

available in established databases [72]. Consequently, in any given application, with wavelength and metal composition appropriately chosen in beforehand, the film's thickness will have the greatest effect on its performance characteristics. Therefore, by varying the film thickness, often only by tens of nanometers, the degree to which each of the bulk physical and optical properties is expressed may be tailored in order to achieve the wide variety of effects listed in Table 2.1 .

For the purposes of this review, the aforementioned application involving metal film-clad optical waveguides, shown in Fig. 2.1, serves as a specific example to demonstrate the significance of accurately controlling and characterizing film thickness in order to tailor the performance characteristics of the waveguide. The waveguides are designed to transmit light directly into the tissue by means of an evanescentleaking effect referred to as optical tunneling. This application sees the use of glass slab substrates as optical light-guides that are designed to restrict the propagation of the visible laser light within their bounding surfaces, specifically $532 \mathrm{~nm}$ from a Q-Switched Nd:YAG laser. The substrates are clad in thin films of titanium and 
silver with a total thickness on the order of $200 \mathrm{~nm}$. Using thin films of metal as a cladding layer guarantees that the waveguides can operate throughout a range of internal reflection angles from $6^{\circ}<\theta_{i}<90^{\circ}$, which can be directly calculated using Snell's Law [66, 90-92].

The waveguides are designed such that the light will reflect within the parallel silver films until reaching an "active area," wherein the silver film's thickness is much thinner, between $0-30 \mathrm{~nm}$, and the light is capable of escaping confinement through optical tunneling, which is comparable to the quantum tunneling experienced by electrons under similar situations. As the laser interacts with the glass-metal interface, a portion of the incident photons within the glass will penetrate into the external medium in the form of an exponentially decaying electromagnetic field, referred to as the evanescent field $[93,94]$. Figure 2.2 demonstrates that in situations wherein the film thickness is shorter than the penetration depth of this field, the peak of the field may protrude through the film and interact with the third external medium. Under such circumstances, the protruding peak may revert back into a propagating field and refract into the tertiary medium, having "optically tunneled" through the metal film [95]. This optical tunneling is utilized in the waveguide application to transmit the laser light into tissue that is in direct contact with the waveguide.

The importance of the silver film thickness in this case relates to the degree to which optical tunneling occurs, as the effect is proportional to the extent to which the evanescent peak protrudes into the external medium, which is correlated to the refractive index and thickness of the cladding film. It has been demonstrated that regulating the thickness of the metal film along the waveguide and within the active 


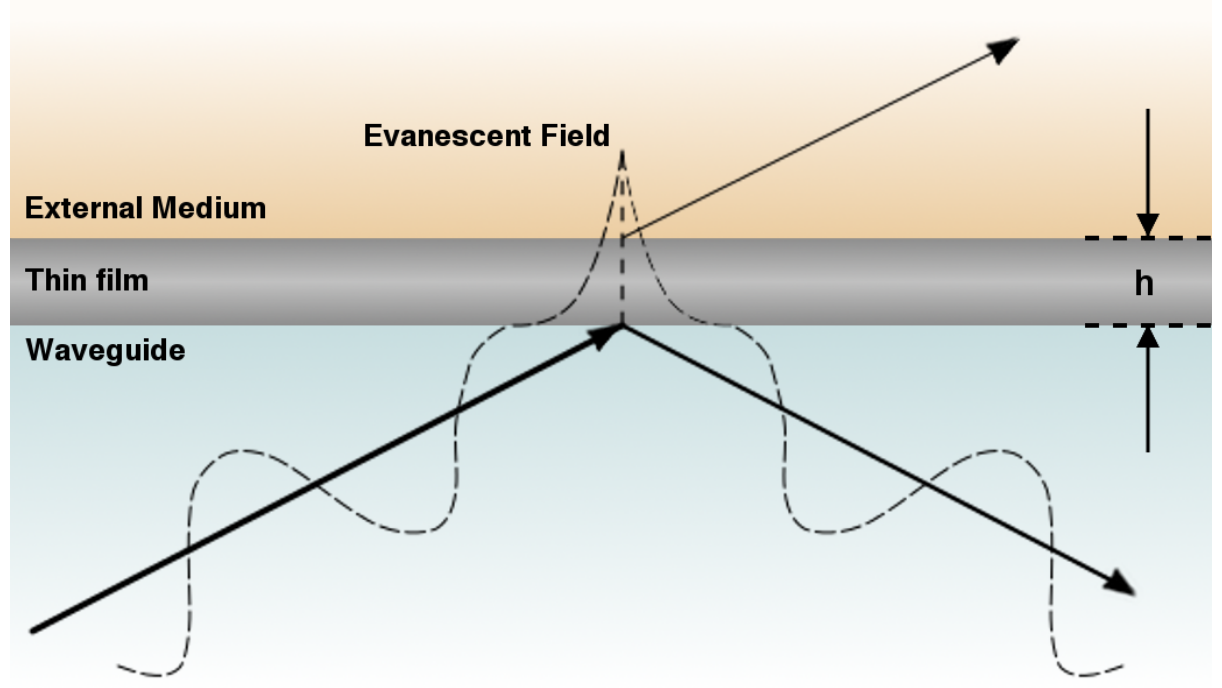

FIGURE 2.2 When a beam of light undergoes total internal reflection, as occurs within optical waveguides, the incident electromagnetic energy slightly protrudes out into the external media at each reflection point; if the cladding layer is sufficiently thin, the light may optically tunnel through the film, resulting in a beam of light within the external medium whose intensity is proportional to the film thickness [66].

area affords control over the location and intensity of energy transmission into the tissue [83]. Consequently, for this example, the accurate characterization of the metal film's thickness is of paramount importance in appropriately exerting control over the beam propagation, both for maintaining confinement within the waveguide and for transmitting the light into the external medium.

\subsubsection{Metal thin film characterization}

Such concerns reverberate throughout the field of metal thin film optics, as the thickness clearly plays such an important role in determining the efficacy of each technology. For the metal clad waveguides, the film thickness determines the degree of transmission into the external medium; whereas for applications in other fields, as in optoelectronics, the film thickness has significant ramifications on conductivity, 
resistivity, and bulk optical absorption as well. However, the majority of metal thin films are deposited in thicknesses much shorter than the wavelength of visible light, and as such are well beyond the diffraction limit of traditional microscopy.

In certain applications, obtaining an estimate of the film thickness within \pm $50 \mathrm{~nm}$ may be sufficient, particularly if the intention is akin to simple mirror fabrication, wherein the principal property of concern is bulk reflectivity. In such cases, older, less complex techniques may be utilized to obtain a functional approximation of the film thickness. Amongst the oldest and most established characterization techniques, UV-Vis spectrophotometry provides a relatively quick and simple analysis of material optical properties, from which bulk film properties can be discerned. Although this technique does not provide the nanoscale resolution offered by other more advanced methods, it is one of the simplest and cheapest characterization methods and can often provide a general measurement of film transmittivity and reflectivity, from which the film thickness may be derived [96]. However, the technique is largely subject to measurement errors and assumptions made in fitting the theoretical equations to the measured interference spectra, resulting in film thickness measurements that may be as much as $200 \mathrm{~nm}$ disparate from measurements produced by more reliable techniques, such as ellipsometry [96, 97]

Consequently, a variety of alternative methods have been established for determining film thickness to a much greater degree of accuracy. It is therefore the purpose of this review to present some of the most predominant characterization methods with respect to their functional theory, methods, and data analysis procedures. This article is intended to serve as a brief introduction to each of the most prominent techniques, 
while highlighting their individual limitations and considerations with regard specifically to the characterizing of metal thin films. In the sections that follow, we provide a cursory introduction to each X-Ray Reflectivity (XRR), Ellipsometry, Atomic Force Microscopy (AFM), cross-sectional Scanning Electron Microscopy (SEM), and SEM coupled with Energy-dispersive X-Ray Spectroscopy (SEM / EDS).

\subsection{Established characterization techniques}

Thin film characterization has long been plagued by the limitations of optical microscopy and contemporary electronics. Although the films themselves have been in consistent use for centuries, their chemical structure and physical geometry had been largely a mystery until the relatively recent development of electronic imaging technologies, albeit many of the original limitations still apply. Nevertheless, the ability to measure and image beyond the diffraction limit of visible light has dramatically improved our understanding of thin film geometry and our capability to closely control thin film fabrication. The sections that follow introduce each of the four aforementioned thin film characterization techniques, covering their respective theories, methods, and analytical methods individually before discussing their principal concerns and restrictions collectively. Table 2.2 lists additional techniques not discussed herein, their most relevant considerations and limitations, vertical resolutions, and accompanying references for the interested reader. 


\begin{tabular}{|c|c|c|c|}
\hline Technique name (abbreviation) & Limitations & $\begin{array}{l}\text { Vertical } \\
\text { Resolution }\end{array}$ & Ref. \\
\hline Atomic Force Microscopy (AFM) & 5 & $0.1 \AA$ & $\begin{array}{l}{[98]} \\
{[99]}\end{array}$ \\
\hline Auger Electron Spectroscopy (AES) & $2,3,7,9$ & $0.5-2.5 \mathrm{~nm}$ & [100] \\
\hline Confocal Microscopy & \multicolumn{2}{|c|}{$1,3,5,6,7,810 \mathrm{~nm}$} & [101] \\
\hline $\begin{array}{l}\text { Cross-Sectional Scanning Electron Microscopy } \\
\text { (SEM) }\end{array}$ & \multicolumn{2}{|c|}{$2,4,6,7,8,9<1 \mathrm{~nm}$} & $\begin{array}{l}{[102]} \\
{[103]}\end{array}$ \\
\hline $\begin{array}{l}\text { Energy-Dispersive X-ray Spectroscopy (EDS) } \\
\text { (i.e. Electron Probe Microanalysis (EPMA)) }\end{array}$ & \multicolumn{2}{|c|}{$1,2,3,4,7,91.5 \mathrm{~nm}$} & $\begin{array}{l}{[104]} \\
{[105]}\end{array}$ \\
\hline Evanescent Field-based Photoacoustics (EFPA) & $1,4,8$ & $38 \mathrm{~nm}$ & [106] \\
\hline $\begin{array}{l}\text { Glow-Discharge Optical Emission Spectroscopy } \\
\text { (GDOES) }\end{array}$ & $1,2,4,7,9$ & $2 \mathrm{~nm}$ & {$[107]$} \\
\hline High Resolution RBS (HR-RBS) & $3,4,9$ & $<1 \mathrm{~nm}$ & {$[110]$} \\
\hline Interferometric Optical Profilometry (OP) & $1,3,4,7,8$ & $0.1 \mathrm{~nm}$ & [101] \\
\hline Medium Energy Ion Scattering (MEIS) & $2,3,4,9$ & $1.5 \AA$ & [111] \\
\hline Raman Spectroscopy (RS) & $1,3,4,7,8$ & $1000 \mathrm{~nm}$ & [112] \\
\hline Rutherford Backscattering Spectrometry (RBS) & $2,3,4,9$ & $5 \mathrm{~nm}$ & [100] \\
\hline Scanning Near-field Optical Microscopy (SNOM) & $1,3,7,8$ & $2-5 \mathrm{~nm}$ & [113] \\
\hline Scanning Tunneling Microscopy (STM) & 2,5 & $0.2 \AA$ & [114] \\
\hline Secondary Ion Mass Spectrometry (SIMS) & $2,3,4,9$ & $0.5-1.5 \mathrm{~nm}$ & [107] \\
\hline Spectroscopic Ellipsometry (SE) & $1,3,4,7,8$ & $0.1 \mathrm{~nm}$ & [115] \\
\hline UV-Vis Spectrophotometry (UV-Vis) & $1,3,4,7,8$ & $1 \mathrm{~nm}$ & [116] \\
\hline X-ray Fluorescence (XRF) & $1,3,5,7$ & $25 \mathrm{~nm}$ & [102] \\
\hline X-ray Photoelectron Spectroscopy (XPS) & $1,3,4,7,9$ & $0.5-2.5 \mathrm{~nm}$ & [100] \\
\hline X-ray Reflectivity (XRR) & $1,3,4,7$ & $1 \mathrm{~nm}$ & [116] \\
\hline
\end{tabular}

TABLE 2.2 A list of techniques that may be used to characterize the thickness of thin films. Measurement limitations are enumerated as follows: (1) Radiative absorption, (2) Sample conductivity, (3) Penetration depth, (4) Computationally derived characteristics, (5) Measurement artifacts, (6) Contrast requirement, (7) Diffraction, (8) Sample characteristics (e.g. refractive index), (9) Requires high vacuum conditions. 


\subsubsection{X-Ray Reflectivity}

The first of the primary technologies used in thin film material characterization, X-ray reflectivity (XRR) operates in a similar manner to UV-Vis reflectance spectrophotometry, with the principal difference that the photons emitted and measured are of substantially shorter wavelength and higher frequency than those in the UV and visible spectra. The use of X-ray photons instead of visible spectrum radiation allows measurement to circumvent inaccuracies caused by optical absorption of visible light while also allowing decreasing the effective diffraction limit. Additionally, whereas traditional spectrophotometry suffers inaccuracies when the observed spectrum deviates from the theoretically expected curve, X-ray reflectivity relies entirely upon deviation from theory in order to derive material properties. The subsections that follow introduce the operational theory and general apparatus design of XRR before discussing the procedure for data analysis and thickness derivation.

\subsubsection{XRR: Theory}

With regard to operational theory, XRR builds on some of the same fundamental optical theories that gave rise to UV-Vis spectrophotometry. In traditional reflectance spectrophotometry, incident light reflects off the interface between the thin film and the air, and again off the interface between the film and the substrate (or the next intermediary layer), forming interference patterns by scanning the sample throughout a range of incident wavelengths. By contrast, X-ray reflectivity functions by scanning the sample surface with a monochromatic beam of X-ray photons through- 
out a range of incident angles $\theta_{i}$. Although the wavelength of the incident photons does not change, the changing incident angle results in variations in the amplitude of the reflected beam, which generates interference patterns throughout the angular spectrum.

Research has demonstrated that typically, the most relevant and useful data will be recorded within a few degrees of the critical angle between the sample and the surrounding atmosphere. Seeing as the same optical principles may be applied to X-ray photons as to visible radiation, the critical angle can be calculated from the simplified form of Snell's law:

$$
\cos \theta_{c}=\frac{n_{2}}{n_{1}}
$$

given that the sample medium is less optically dense than the surrounding medium at the X-ray wavelength (i.e. $n_{2}<n_{1}$ ) [117]. As with visible optics, angles of incidence less than $\theta_{c}$ will result in a portion of the incident beam refracting into the medium, while the remaining portion of the beam reflects away; whereas angles greater than $\theta_{c}$ will result in the incident beam to totally reflecting away from the interface. Consequently, scanning through the range of angles surrounding the critical angle yields multiple beam scenarios such as are depicted in Fig. 2.3.

However, the true strength of the technique is derived from the extremely short wavelengths of X-ray photons, which permits them to interact with materials on an atomic level. As a result, the incident beam is strongly susceptible to the changes in electron density that occur at the interfaces between media of differing refractive indices, due to the strong dependence of refractive index on electron density. As the 


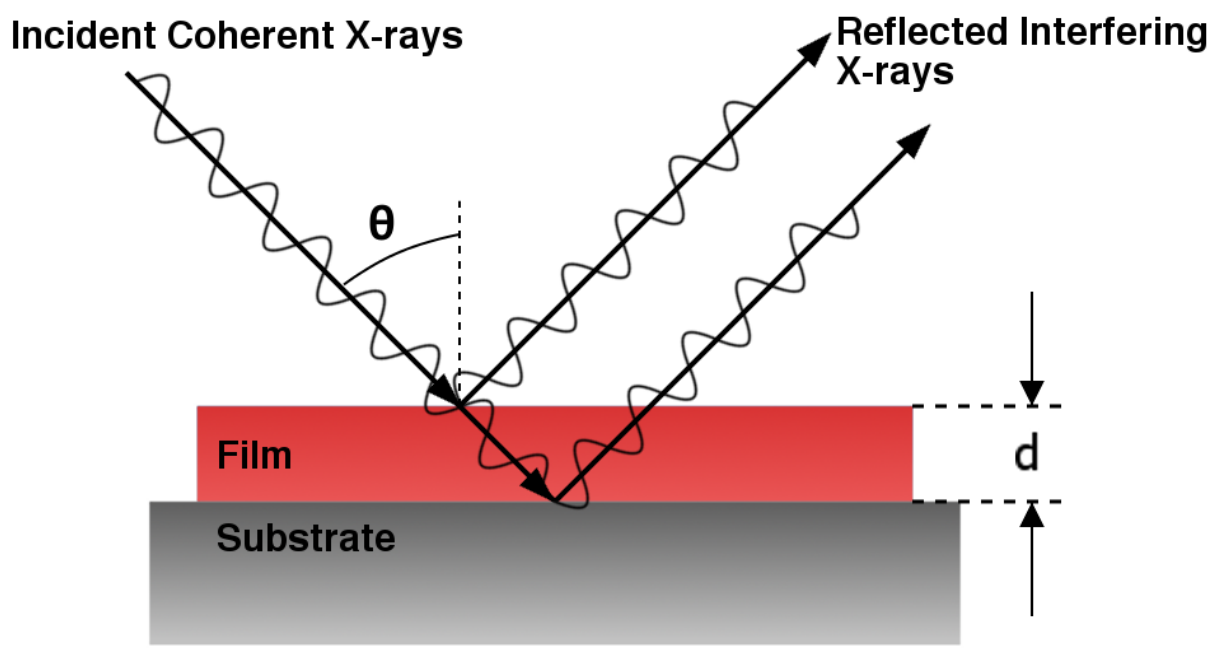

FIgURE 2.3 An incident beam of X-ray radiation may reflect or transmit through a material in much the same way as a coherent laser beam. By measuring the coherence of the reflected beams and the degree of reflectivity throughout a range of incident angles $\theta$, it is possible to computationally derive material properties.

incident angle changes, the X-rays reflecting off the surface will interfere with the $\mathrm{X}$-rays that penetrate the sample and reflect off the sample-substrate interface. This interference results in constructive and destructive peaks and valleys in the measured spectra. Therefore, by recording the amplitude of the specularly reflected X-rays and their coherence, a parameterized model can be fit to the data to incorporate electron density $\rho_{e}$, thickness $d$, and surface roughness $r$. Although the parameterized model can reveal more specific information about the sample, a quick analysis reveals that the periodicity of the peaks is proportional to $2 \pi / d$, whereas the amplitude is proportional to the density difference [117]. Moreover, more complex models can be implemented for cases involving multilayered structures, such as the fused silica titanium - silver multilayered waveguides discussed in Section 2.1.1.

$$
\frac{R(Q)}{R_{\text {Fresnel }}(Q)}=\left[\frac{1}{\rho_{\infty}} \int_{-\infty}^{+\infty} e^{\mathrm{i} Q z}\left(\frac{d \rho_{e}}{d z}\right) d z\right]^{2}
$$


Equation 2.2 represents the ratio of the measured reflectivity, $R(Q)$, over the ideal Fresnel reflectivity, $R_{\text {Fresnel }}(Q)$, which is equal to the average electron density profile as a function of depth, $z$ [118]. In this case $\rho_{e}$ is the electron density and $Q$ is the X-ray wave vector given by:

$$
Q=\frac{4 \pi \sin \theta_{i}}{\lambda}
$$

wherein $\theta_{i}$ is the angle of incidence and $\lambda$ is the wavelength of the X-ray photon. In order to derive the film thickness, the parameters of the electron density model are computationally optimized to better fit the reflectance ratio spectrum. Such an analysis yields a parameterized model that varies with increasing depth, and therefore an electron density profile that yields the positions of each interface, from which the film thickness may be derived. An additional benefit may come from scanning the beam of x-rays across the surface of the film, which can reveal information regarding surface film homogeneity [119].

\subsubsection{XRR: Method}

With regard to operational methods, a goniometer is used to scan the x-ray emitter and detector throughout a range of incident angles, both of which may be moved independently with respect to the stationary sample stage, as shown in Fig. 2.4. As the incident beam transmits through the thin film, a portion of the beam will reflect at each subsequent interface: air-film, film-film, and film-substrate. As mentioned above, these reflections are due to variations in local electron density, dependent upon 


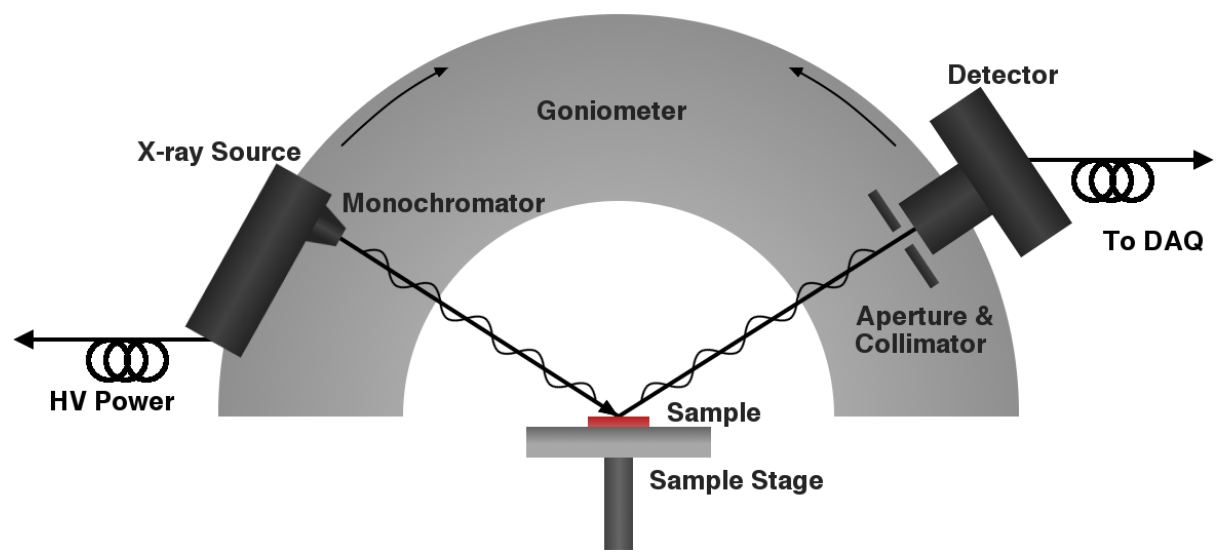

FiguRE 2.4 The X-ray source and detector are attached to a goniometer that allows for consistent, relative rotation with respect to the sample surface so that the detector may always be positioned such that only the specularly reflected beam is considered. The XRR often incorporates a monochromator, entrance aperture, and collimator to improve the reliability of the system.

local material composition, which aids in distinguishing between individual layers.

The equipment for generating X-rays is identical to a standard X-ray diffraction device (XRD), although the X-ray source may need to be replaced to produce slightly longer wavelengths. As such, a parallel beam of monochromatic, coherent X-rays is shone upon the sample surface, however the detector is oriented such that it detects only those X-rays that are specularly reflecting off the surface; i.e. the detector geometry is designed such that diffusely reflected X-rays are not detected or are computationally ignored. A goniometer is also utilized to move the X-ray source and detector in an arc centered around the sample, such that the sample may be interrogated through an angular spectrum while guaranteeing only the specularly reflected photons are detected. Often, the detector will be designed to incorporate a specific entrance aperture or slits to prevent measurements generated by diffusely reflected X-rays from affecting the measured interference patterns. 


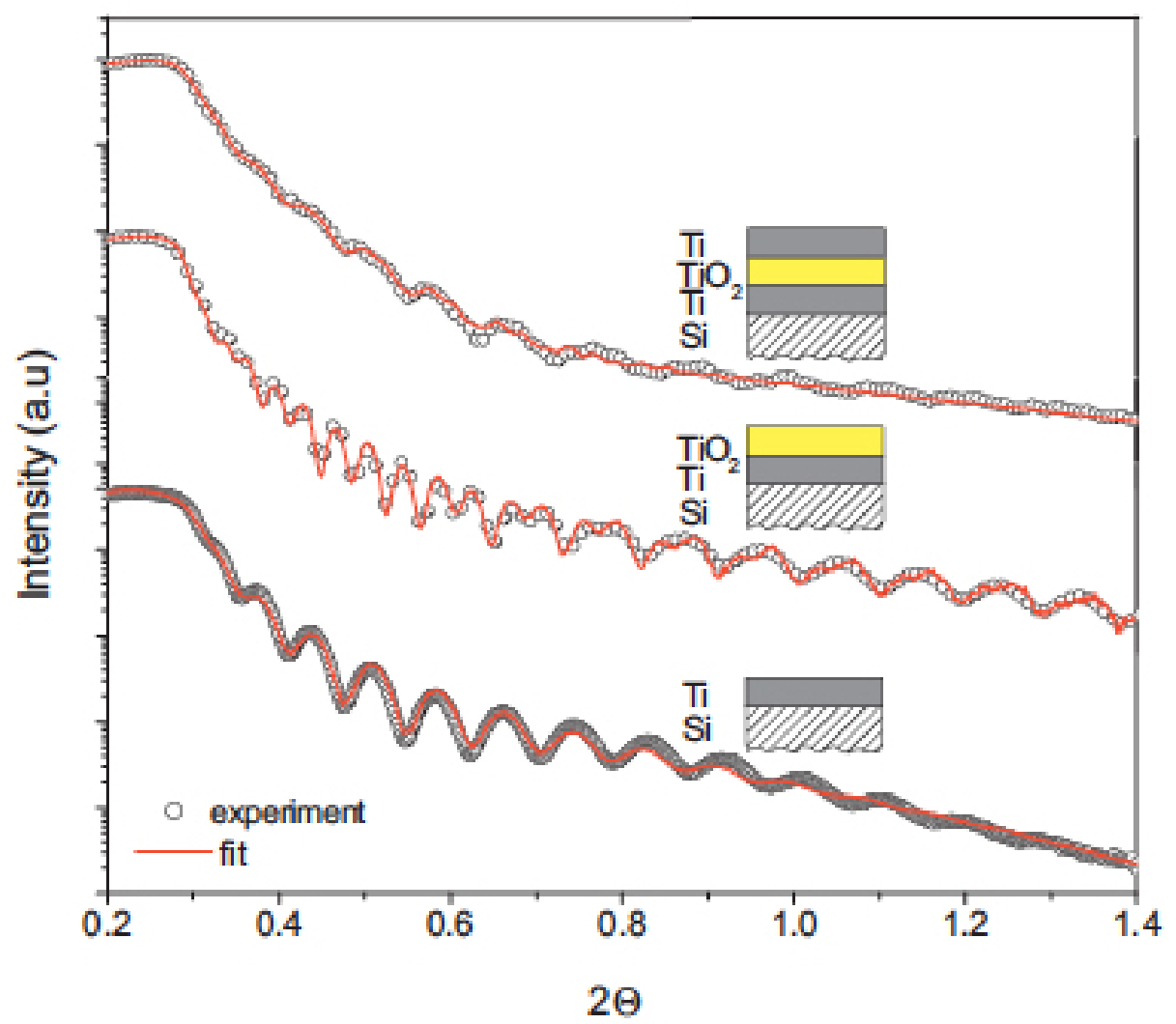

FIGURE 2.5 By fitting parameterized equations to each of the three x-ray reflectivity data traces above, it can be determined that the base Ti layer is $47 \mathrm{~nm}$ thick on the lowest trace, both the $\mathrm{Ti}$ and $\mathrm{TiO} 2$ layers are $46 \mathrm{~nm}$ thick in the center trace, and the three layers on the top trace are $40 \mathrm{~nm}, 51 \mathrm{~nm}$, and $49 \mathrm{~nm}$ respectively on the top trace, demonstrating a distinct decrease in accuracy with increasing film thickness. [Reprinted from Materials Research Bulletin, 47, 2, K. Drogowska, et al. , "RBS, XRR and optical reflectivity measurements of $\mathrm{Ti}-\mathrm{TiO}_{2}$ thin films deposited by magnetron sputtering", Page 299, Copyright (2012), with permission from Elsevier]

\subsubsection{XRR: Analysis}

In another similarity with spectrophotometry, the film thickness and other physical properties are not directly measured, but are instead computationally derived by comparing a parameterized model of the electron density profile to the ratio of measured reflectivity over the Fresnel reflectivity (Eq. 2.2). To demonstrate the computational analysis process, a sample set of interference spectra is included in Fig. 2.5, which 
is a combined graph of three independent sample traces for titanium and titanium dioxide films deposited on silicon substrates in approximately $50 \mathrm{~nm}$ layers [116]. The data is fit in each case with a parameterized equation that yielded the thickness, roughness, and density for each film involved. However, it should be noted that as the number of layers increased, the number of necessary parameters also increased, and the resultant fit became less accurate. Additionally, the similarities in electron densities between layers of $\mathrm{Ti}$ and $\mathrm{TiO}_{2}$ yield less distinct interference peaks than are generated by the differing densities at the $\mathrm{Si}-\mathrm{Ti}$ layer on the bottom of the intensity plot.

Nevertheless, with regard to the thickness, the measurements based on the single film layer were most accurate, with a margin of error of only $\pm 1 \mathrm{~nm}$; whereas, for the triple film layer sample, the margin of error increased to $\pm 2 \mathrm{~nm}$ for each film thickness. The plot also demonstrates that as the overall film thickness increases, the interference intensity curve undulates to a lesser extent, which lessens the accuracy of the parameterized fitting functions. Consequently, the increase in error observed in the multi-layer films is directly resultant from their collective thickness.

Additionally, although the equipment measured the same film with subsequent depositions, the measurements for each of the films changed as additional films were deposited on top. This is to be expected, as the mean free path of the photon is insufficient to accurately measure films beyond approximately $100 \mathrm{~nm}$. XRR relies on the penetration and subsequent reflection of X-ray photons at the interfaces between material boundaries; however, with increasing sample thickness, the incident photons stand a greater chance of absorption, scattering, or diffuse reflection, all of which 
diminish the specular reflectance measurements and thereby decreases the accuracy of the parameterized fit [117]. Nevertheless, the accuracy of the fit for ultra-thin films on the order of $25 \mathrm{~nm}$ is extremely good, with measurement error falling to around only $\pm 0.3 \mathrm{~nm}[120]$. As such, in the course of metal film characterization, if the film thickness is known to be less than $100 \mathrm{~nm}$, XRR is likely to produce reliable measurements of film thickness; although, it will not yield information about properties of the film in the visible spectrum.

\subsubsection{Ellipsometry}

Another optically-based characterization technique, ellipsometry was initially established 100 years ago by Paul Drude in Germany; however, it was only with the advent of modern computing and phase modulation that the true strength of the technique as a characterization tool was demonstrated [121]. As with spectrophotometry, ellipsometry uses visible light to derive thin film properties; however, the analysis is much more accurate as it incorporates changes in both reflected amplitude and phase. As above, the subsections that follow introduce the operational theory behind ellipsometry, the methods for its use, and the procedure for obtaining film thickness measurements from the resultant data.

\subsubsection{Ellipsometry: Theory}

Ellipsometry builds on many of the same principles that were established by spectrophotometry, but more acutely measures the changes that result following reflection of the incident beam of photons off each interface. The technique utilizes linearly po- 
larized visible light, which may also be scanned through a spectrum of wavelengths, to illuminate a sample surface at a designated angle of incidence, $\theta$. Under such situations, when the polarized light intersects the interface, as with the X-rays discussed earlier, a portion of the beam will reflect away from the interface, whereas the remaining portion will transmit down to the next interface. The strength of ellipsometry, however, is derived from the amplitude of the reflected beams along with the phase shift that occurs as the beam refracts and reflects within each layer.

Building on that foundation, the linearly polarized light can be considered to be a product of separate transverse magnetic (TM or "p-polarized") and transverse electric (TE or "s-polarized") components. The Fresnel equations, shown in Equations 2.4 and 2.5, express the reflection coefficient for each of these conditions at the interface of two media:

$$
\begin{aligned}
& \rho_{\pi}=\left(\frac{\tilde{n}_{2} \cos \theta_{1}-\tilde{n}_{1} \cos \theta_{2}}{\tilde{n}_{2} \cos \theta_{1}+\tilde{n}_{1} \cos \theta_{2}}\right) \\
& \rho_{\sigma}=\left(\frac{\tilde{n}_{1} \cos \theta_{1}-\tilde{n}_{2} \cos \theta_{2}}{\tilde{n}_{1} \cos \theta_{1}+\tilde{n}_{2} \cos \theta_{2}}\right)
\end{aligned}
$$

wherein $\rho_{\pi}$ and $\rho_{\sigma}$ are the coefficients for each the p- and s-polarizations respectively, $\tilde{n}_{1}$ and $\tilde{n}_{2}$ are the complex refractive indices for the incident and secondary media, and $\theta_{1}$ and $\theta_{2}$ are the angles of incidence and refraction.

Given that the thin films in question are deposited on a substrate layer, or may incorporate multiple consecutive layers, the reflection coefficients for each subsequent layer must all be incorporated into the total reflection coefficient. The expressions for a simple three-layer system (e.g. air-film-substrate) are provided in Equations 
2.6 and 2.7 below:

$$
\begin{aligned}
& P_{\pi}=\left(\frac{\rho_{12, \pi}+\rho_{23, \pi} e^{-\mathrm{i} 2 \beta}}{1+\rho_{12, \pi} \rho_{23, \pi} e^{-\mathrm{i} 2 \beta}}\right) \\
& P_{\sigma}=\left(\frac{\rho_{12, \sigma}+\rho_{23, \sigma} e^{-\mathrm{i} 2 \beta}}{1+\rho_{12, \sigma} \rho_{23, \sigma} e^{-\mathrm{i} 2 \beta}}\right)
\end{aligned}
$$

For these equations, the reflection coefficients at each boundary are referred to as $\rho_{n m}$, where $n$ is the incident medium and $m$ is the refracting medium. Additionally, $\beta$ is incorporated as an expression of the phase change that occurs from the top to the bottom of each layer:

$$
\beta=2 \pi \frac{d}{\lambda} \tilde{n}_{2} \cos \theta_{2}
$$

which is dependent upon the wavelength $\lambda$, refractive index of the refracting medium $\tilde{n}_{2}$, angle of refraction through the medium $\theta_{2}$, and the film thickness $d$ [122]. Con-

sequently, by measuring the phase shift and reduction in amplitude of the reflected beams relative to the known and controlled light source, computational analysis may be performed to determine a value for $\beta$ and thereby derive the complex refractive index and film thickness of each layer.

\subsubsection{Ellipsometry: Method}

Modern ellipsometers typically contain a specific series of specialized optical components in order to accurately track the effects on a beam of light resultant from reflection off a multilayered surface. Figure 2.6 depicts the components necessary for properly performing ellipsometric analysis on a thin film sample, which may be 


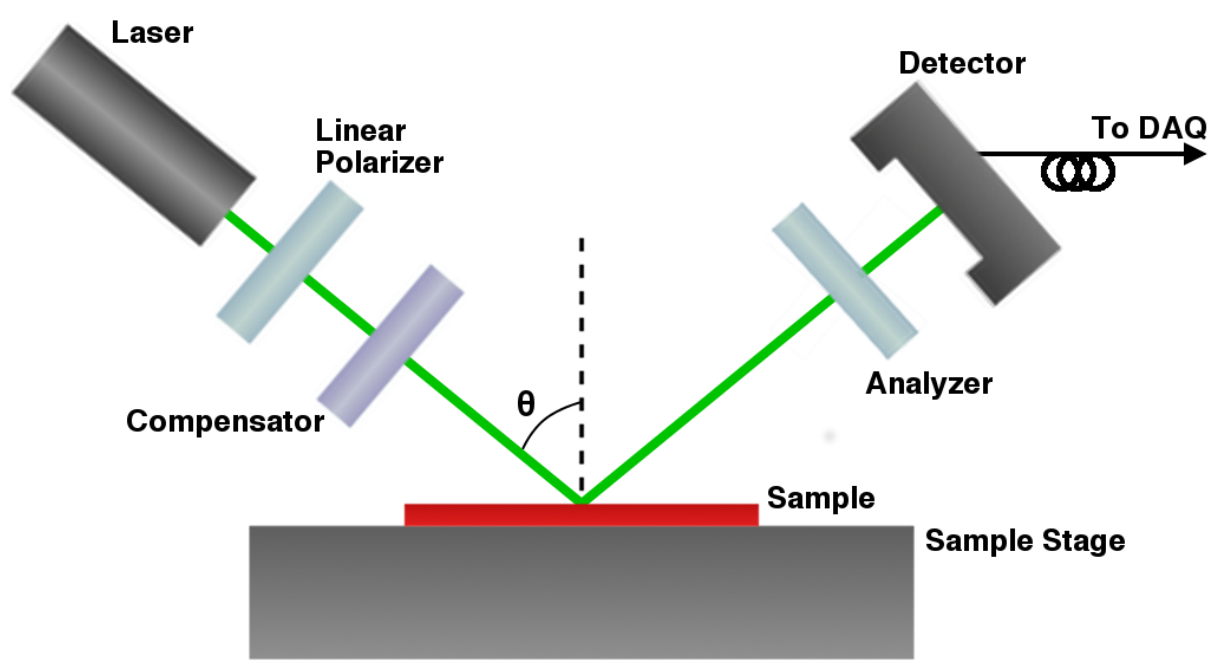

FIGURE 2.6 A collimated light source outputs a monochromatic beam through a linear polarizer and compensator (optional) toward the surface of a sample. The polarization of the reflected beam is rotated upon interaction with the sample, which can be measured by an analyzer and detector pair, and is indicative of material properties such as film thickness and refractive index.

modified to include additional components as needed. However, regardless of the application, the system will typically proceed along the same progression of optical components. First, a collimated light source generates a beam of light, which is transmitted through a linear polarizer and subsequently passed through a compensator, which is typically either an electronic phase modulator or quarter-wave plate. The purpose of these components is to afford control over the incident polarization state of the light, so as to accurately measure the phase change upon reflection.

The final components incorporate an analyzer and a detector pair, which allow for independent measurement of the resultant wavelength-dependent phase shift and reduction in amplitude resultant from reflection. Additionally, the components are typically also mounted on a goniometer, which allows for the incident reflection angle to be modified between spectra. Based purely on the optical theories presented in 
the previous section, it is possible to derive the film thickness using either a single wavelength throughout a spectrum of angles, or alternatively a spectrum of wavelengths operating at a single reflection angle; however, due to the strong dependence of refractive index and optical absorption on wavelength, in addition to the effects of chromatic dispersion, it is often advisable to perform multiple wavelength spectra at various angles of incidence to reduce measurement errors.

Most modern ellipsometers utilize software that is designed to fit a parameterized model to a spectrum of wavelengths as opposed to a spectrum of angles; although, it may also be beneficial to record measurements at a variety of incident angles and average the derived values for each fit. The spectra typically range from the upper end of the UV to the lower end of the near-Infrared regions. For applications involving metal films, it should be noted that UV light tends to be largely absorbed by most metals, and as such shorter wavelengths are not ideal for producing reliable data. Additionally, infrared wavelengths tend to be absorbed by adsorbed water molecules or other organic molecules in the local atmosphere, which may add measurement error to the resultant film thickness calculations; although, applications for infrared wavelengths in characterizing certain metamaterials and organic films have been demonstrated [123].

\subsubsection{Ellipsometry: Analysis}

After recording the reflected intensity and the phase change for both s- and ppolarizations for each wavelength in the spectrum, the ellipsometry software will create a complex reflectance ratio of the reflectivity for p-polarized light, $P_{\pi}$, divided 

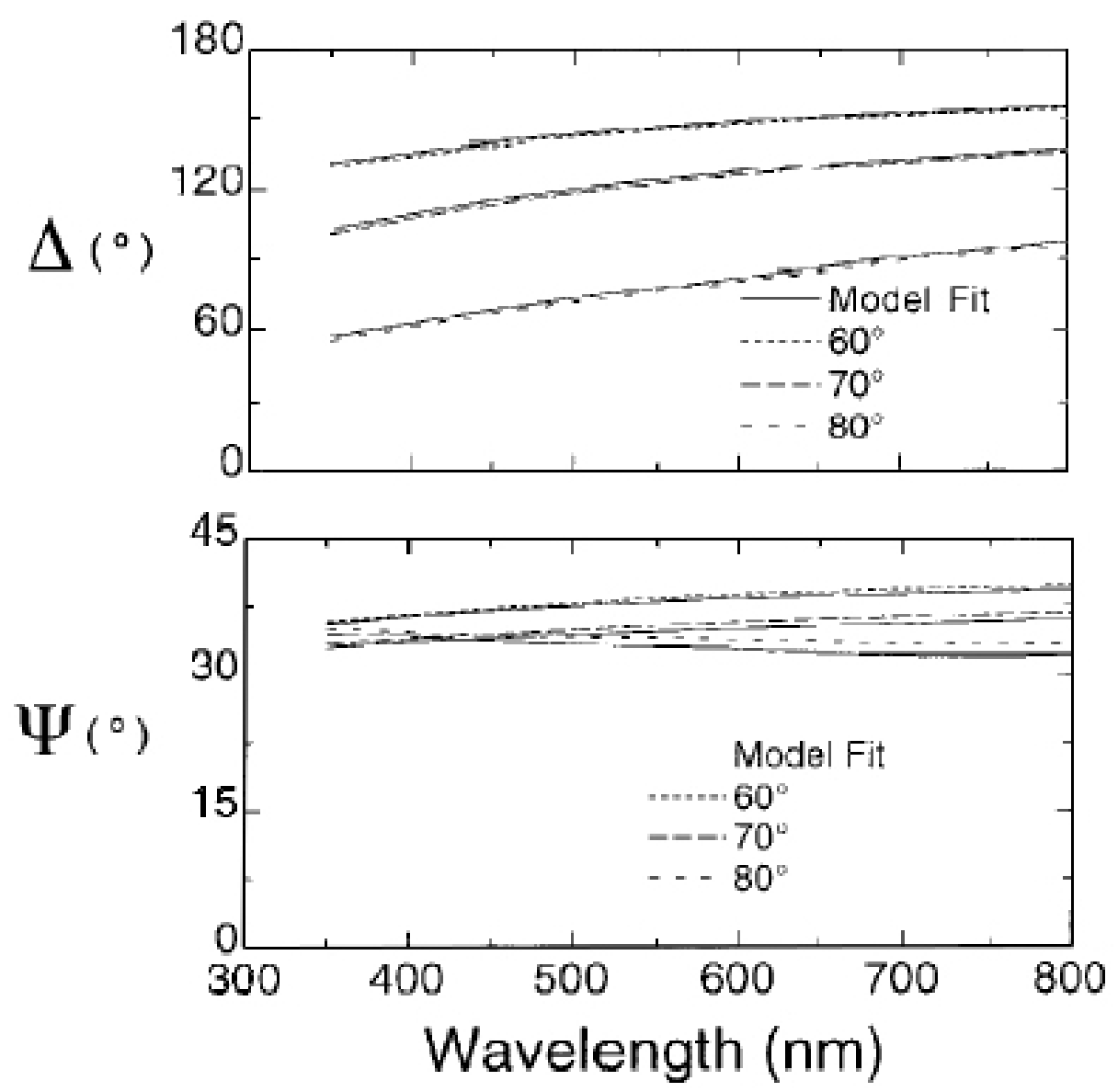

FIGURE 2.7 Full wavelength spectra were recorded for three incident angles of light upon platinum films deposited on silicon wafers. Applying the model fits to each spectrum determined that the film thickness was derived to be $65.3 \mathrm{~nm}$. [Reprinted from Surface and Interface Analysis, 29, H.G. Tompkins, et al. , "Spectroscopic ellipsometry measurements of thin metal films", Page 185, Copyright (2000), with permission from John Wiley and Sons, Ltd.]

by that of the s-polarized light, $P_{\sigma}$, given by Equations 2.6 and 2.7 respectively. This ratio can also be expressed as:

$$
P=\frac{P_{\pi}}{P_{\sigma}}=\tan (\Psi) e^{\mathrm{i} \Delta}
$$

where $\tan (\Psi)$ represents the amplitude reflectance ratio of the two polarizations and $\Delta$ represents the phase change [124]. As was the case with spectrophotometry, since 
the optical properties are wavelength dependent and are derived alongside the film thickness, ellipsometry uses a spectrum of incident wavelengths in order to determine these values. The principal difference between the two is that by observing the change in phase in addition to the change in amplitude, the parameterized model takes a two-tiered approach dependent upon two independent variables and consequently produces more accurate results.

However, in order to retrieve the thickness and refractive index values from this relationship, the ratio spectrum must be fit to an appropriate model, as shown in Fig. 2.7. Often, the model selection comes down to the preference of the user, although it is typically advisable to take the "simpler, better" approach, unless there is reason to involve additional variables [125]. It is important to note that this model will not be an exact fit to the data, particularly for multilayered, thicker, or more optically absorptive metal thin films.

For example, since optical absorption is wavelength dependent, the deviation from theoretically predicted values will also change based on the incident wavelength. Although the theoretical equations do incorporate the complex component of the refractive index into the calculations, it is challenging to separate amplitude loss due to absorption from that due to transmission or scattering. Additionally, the optical penetration depth of a photon into a material is a function of wavelength, which would suggest that longer wavelengths will penetrate more deeply into the material, which may alter the accuracy with which a film's thickness is measured. Consequently, there will likely be a significant degree of error in the thickness and refractive index values output by the software for metal and other optically absorptive thin films. 
Additionally, this error compounds with increasing film thickness, so much so that many ellipsometer manufacturers advise against utilizing ellipsometry for films thicker than $50 \mathrm{~nm}$, as the values may no longer be considered reliable.

\subsubsection{Atomic Force Microscopy}

The field of profilometry encompasses a wide variety of techniques utilized for surface topographical analysis with sub-nanometer resolution. Although Scanning

Probe Microscopy (SPM) and Scanning Tunneling Microscopy (STM) may be used in certain cases for the analysis of thin film thickness, they necessitate that the sample be electrically conductive; and, whereas the metal films in question are indeed conductive, they it is common for them to be deposited on insulating glass or ceramic substrates [126]. As such, Atomic Force Microscopy (AFM) is typically utilized instead, since it measures mechanical tip deflection rather than electrical current and consequently is not subject to such electrical limitations [127].

\subsubsection{AFM: Theory}

Unlike other surface analysis methods, AFM measures the surface profile and film thickness through direct correlation with a mechanical action, as opposed to calculating derived values by data-fitting a theoretical model. Whereas the previously discussed techniques have each been based on various forms of radiative reflection, this technique functions by directly measuring the deflection and corresponding oscillation of a flexible, microscopic cantilever tip caused by attractive and repulsive atomic forces experienced as the tip is moved vertically with respect to the film's sur- 
face. At relatively long distances, the attractive van der Waals forces will dominate the action of the tip, resulting in a characteristic deflection toward the surface relative to the strength of the force, as can be seen in Fig. 2.8 when the approaching tip makes contact. Following contact, however, the repulsive Coulombic forces will begin to dominate, causing an opposing deflection, which is measured in the increasing $\mathrm{Z}$ voltage. As the tip is retracted away from the surface, the attractive forces begin to dominate again and hold on to the tip, resulting in an inversely concave deflection until reaching a threshold at which the forces are no longer sufficient to hold the tip and the voltage returns to baseline. Moreover, by moving the tip within the transition region between attractive and repulsive forces, the these forces can generate a springlike oscillation in the cantilever, whose frequency is characteristic of the surface being analyzed.

In order to achieve high resolution, the cantilever tip must be manufactured to be only a few nanometers thick at its tip. However, because the oscillations are resultant from atomic forces, AFM does not necessitate that the sample be either conductive, as is the case with Scanning Probe Microscopy and Scanning Tunneling Microscopy, or optically reflective or transparent, as is the case with light-based methods. Additionally, since the tip effectively measures the strength of atomic forces, AFM is capable of characterizing surface properties in addition to surface geometry. Moreover, AFM may be utilized to generate a three-dimensional surface profile by raster scanning the surface with the cantilever and recording the deflection with respect to the horizontal grid system. In this manner, a 3D reconstruction of surface geometries may be computationally reconstructed; however, in order to specifically measure the 


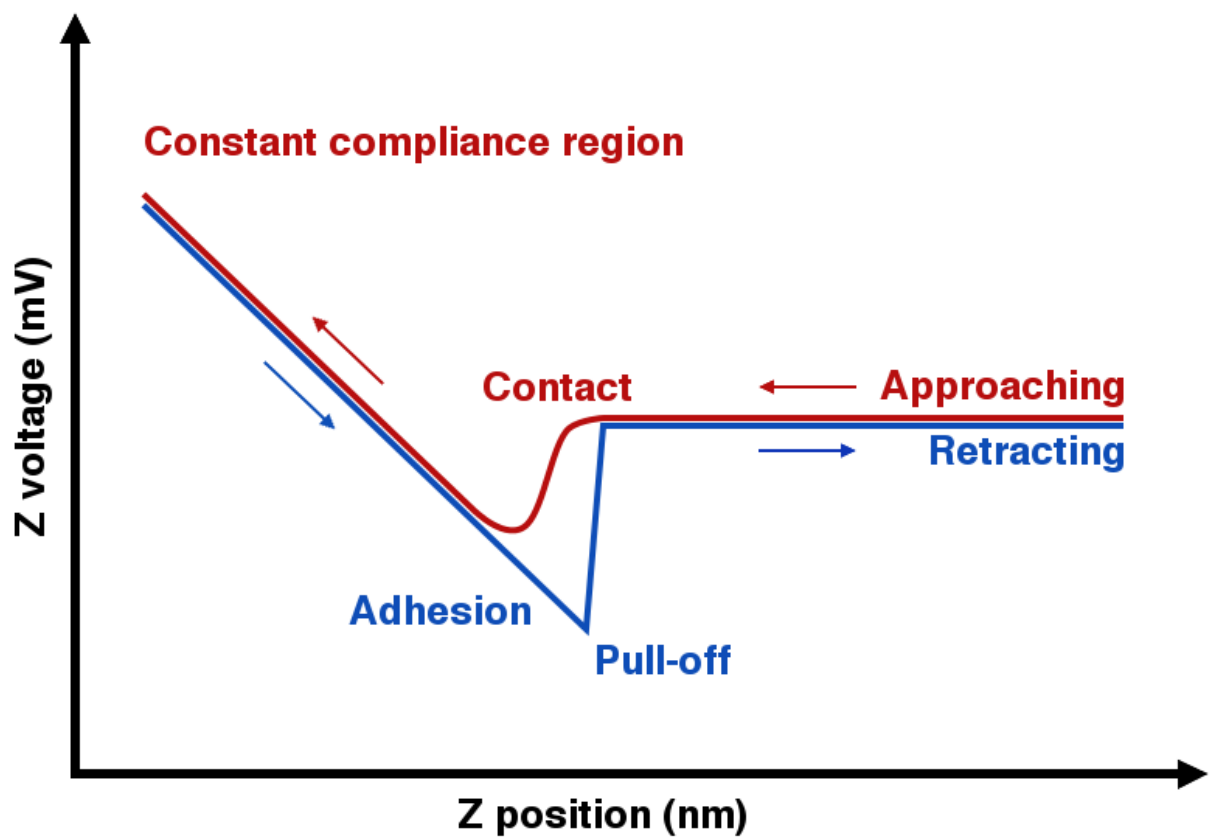

FigURE 2.8 The measured voltage, which corresponds to cantilever deflection, decreases briefly at the moment of contact, before increasing with tip deflection while in constant contact with the sample. When retracting, the attractive intermolecular forces cause the tip to deflect toward the sample until reaching an adhesion threshold and releasing the cantilever back to baseline.

thickness of a thin film, it is necessary to raster the tip across a transition region, wherein the discrepancy in the vertical position of the film's surface and the substrate layer may be measured, such as a border or furrow. The waveguides discussed in Section 2.1.1 are fabricated with masked-off active areas, which when removed expose the underlying substrate material and provide a significant transitionary region between the substrate and metal-film cladded sections.

However, in cases wherein the film is homogeneously deposited across the substrate surface, another method for measuring the thickness with AFM is to scratch the film and measure the depth of the scratch [128]. For sufficiently thin films, the scratch will penetrate down to the substrate and result in an accurate thickness measurement; however, care should be taken with this approach as an insufficiently deep 


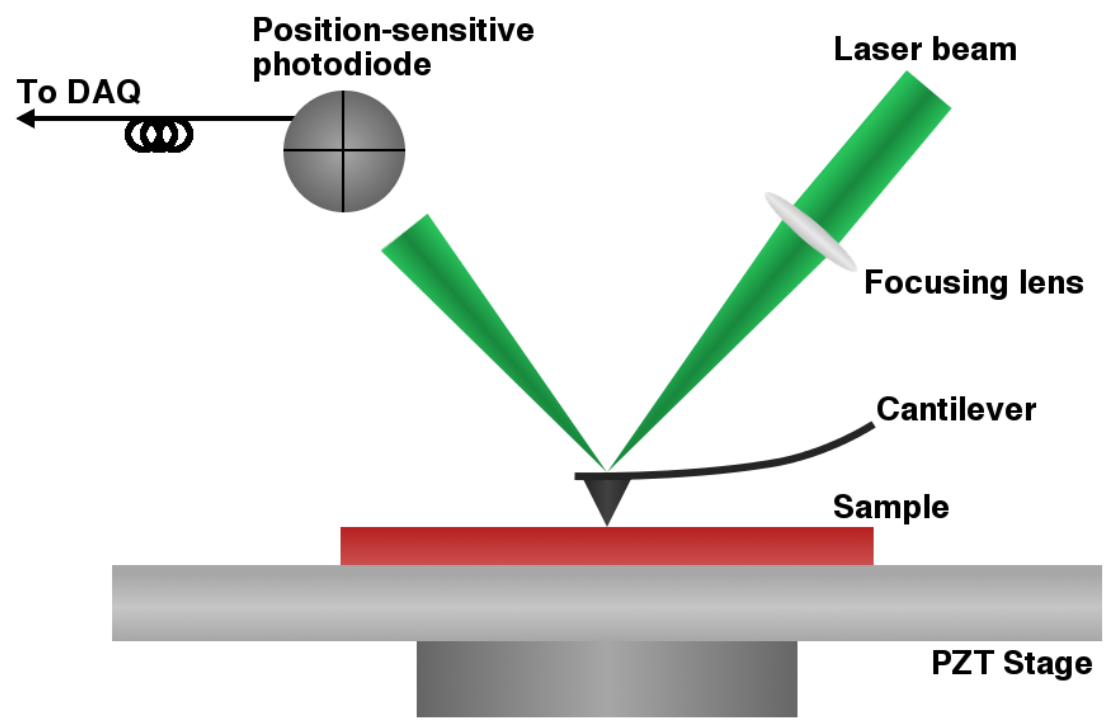

FIGURE 2.9 AFM equipment utilizes a laser beam deflected off the rear face of a microscopic cantilever to measure the tip deflections resultant from intermolecular forces acting on the nano-scale tip using a position-sensitive photodiode and data acquisition unit (DAQ).

scratch will not yield a measurement of film thickness, but instead simply a measure of the depth of the scratch in the film. Moreover, if no transition occurs by design, the scratch method necessitates damaging the surface of the sample and is no longer a completely non-destructive technique. Additionally, if the cantilever tip is used to generate the furrow in the film, it must also be resilient enough to subsequently scan the film; AFM tips sputter coated with titanium nitride have been demonstrated to be effective at such a task [129].

\subsubsection{AFM: Method}

The method of operation for an AFM follows directly from the theory, requiring few components beyond the cantilever, tip, and scanner. In addition, typical AFM systems incorporate a laser source, which focuses a beam of light onto the rear face of the cantilever tip, and a photodetector, which analyzes the intensity of the beam 
reflected off the tip, as shown in Fig. 2.9. The apparatus may also include a voltmeter or an arbitrary waveform generator in order to either measure the oscillation of the cantilever or drive the oscillation with an applied waveform.

The AFM system may be operated in contact mode, wherein the tip remains in contact with the sample surface throughout testing, or tapping mode, wherein the spring-like undulation of the cantilever tip is used to measure surface properties based on attractive and repulsive forces. Furthermore, the apparatus may be designed such that the piezoelectric translational stage (PZT) moves the sample stage with respect to a fixed cantilever setup, or such that the cantilever and laser setup is moved with respect to a fixed sample stage; however, both orientations may produce comparable results. It should be noted that hysteresis in the piezoelectrics may lead to measurement artifacts and non-linear, inconsistent lateral resolution. However, for the purposes of film thickness characterization, as long as there is sufficient resolution at the transition between film and substrate, the thickness should be accurately measured. Additionally, if the cantilever tip is in motion across the surface, it is recommended to operate in contact mode to avoid the addition of any vibrations from the scanning action [114].

\subsubsection{AFM: Analysis}

AFM analysis is much more straightforward than other techniques mentioned herein, since the reflected laser intensity directly corresponds to the position of the tip relative to the surface, assuming the system has been properly calibrated. Although only a single pass over the transition between film and substrate is necessary to measure 


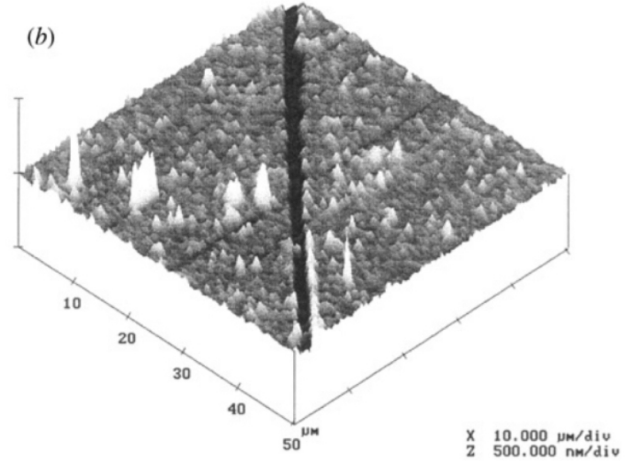

(a) 3D Surface scan

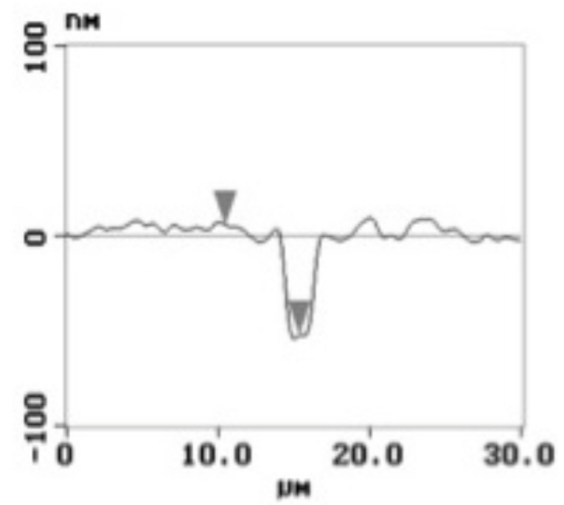

(b) Cross-sectional analysis

FIGURE 2.10 An AFM cantilever tip was used to scratch a furrow in a 14-bilayer polymerpolyelectrolyte film on a silicon substrate. Cross-sectional analysis revealed the depth of the furrow, and therefore the thickness of the film, to be approximately $54 \mathrm{~nm}$. [Reprinted from Nanotechnology, 14, R. Lobo, et al. , "In situ thickness measurements of ultra-thin multilayer polymer films by atomic force microscopy", Page 392, Copyright (1999), with permission from IOP Publishing]

depth, typically a full two-dimensional scan will be performed in order to generate a three-dimensional surface profile, such as that shown in Fig. 2.10. Scanning over a large area also ensures that variations, artifacts, and debris on the film surface do not distort thickness measurements.

Film analysis in such a manner will not only reveal the thickness of the film, but also aspects of the transition area like steepness and film homogeneity. For example, the study that recorded the AFM image shown in Fig. 2.10 also noted that in the course of scratching the furrow in the polymer film, the cantilever tip created ridges along one edge where the polymer had been pushed to one side. In order to compensate and accurately determine the thickness of the film, they scanned over a relatively large area $\left(2500 \mathrm{\mu m}^{2}\right)$, so that the baseline used as a top surface reference would not be distorted by the ridge height.

By scanning the cantilever tip across the furrow, the researchers were able to 
generate the cross-sectional profile shown in Fig. 2.10, which determined the thickness of the 14-bilayer film to be approximately $54 \mathrm{~nm}$. Although the specific value is not quoted in the text, a graph of the number of bilayers plotted against the film thickness recorded by AFM was presented in the text, which demonstrated a linear relationship between the two. That said, typical AFM systems have a sub-nanometer vertical resolution, so as long as the transition between the substrate and the film surface is being observed, the thickness should be readily determined with a relatively low degree of error. It should also be noted that such measurements using a furrow to measure thickness will be fundamentally limited by the sharpness of the cantilever tip. Moreover, although AFM can indirectly measure the thickness of a thin film on a substrate without the need for complex curve-fitting, the technique provides little other information beyond thickness and homogeneity. Consequently, in relating

optical transmission to metal film thickness, AFM is only capable of completing half of the analysis and so must be used in conjunction with an optically based technique in order to complete the analysis.

\subsubsection{Electron Beam Techniques}

Electron beam based technologies represent a categorically different alternative to traditional techniques that rely on electromagnetic radiation to interrogate sample surfaces. Although there are a number of different techniques that have spawned from e-beam approaches, including Auger Electron Spectroscopy (AES) and Transmission Electron Microscopy (TEM), two technologies have demonstrated particular application in determining thin film thickness: cross-sectional Scanning Electron Microscopy 
(SEM) and Energy Dispersive X-ray Spectroscopy (EDS or EDX). The former technique uses secondary generated electrons to computationally produce a two dimensional image of a film cross-section, which may provide a clear depiction of the film sitting atop the underlying substrate layer. By contrast, the latter technique uses X-rays generated by the incident e-beam to characterize the elemental composition of the film, which may be compared to the elemental composition of the substrate to computationally determine the film thickness.

\subsubsection{E-Beam Techniques: Theory}

Both of the techniques mentioned may be performed using an SEM system, although the SEM-EDS necessitates a suitable X-ray detector in addition to the standard secondary electron detector used in SEM image generation. In both cross-sectional SEM and SEM/EDS analysis, a beam of electrons is directed in a column incident upon the sample surface, generating a variety of effects that are strongly correlated with the energy of the e-beam, each of which provide an array of information about the sample and can be utilized to determine a variety of its characteristics. For example, backscattered electrons may be used to determine sample crystallography; whereas Auger electrons may be detected to provide information about the surface composition. Figure 2.11 depicts the characteristically bulbous electron beam interaction volume and demonstrates the various depths at which each effect is generated.

However, cross-sectional SEM analysis is principally concerned with the topography of the film cross-section, and so utilizes the detection of secondary electrons generated within the sample through inelastic scattering. Although these electrons 


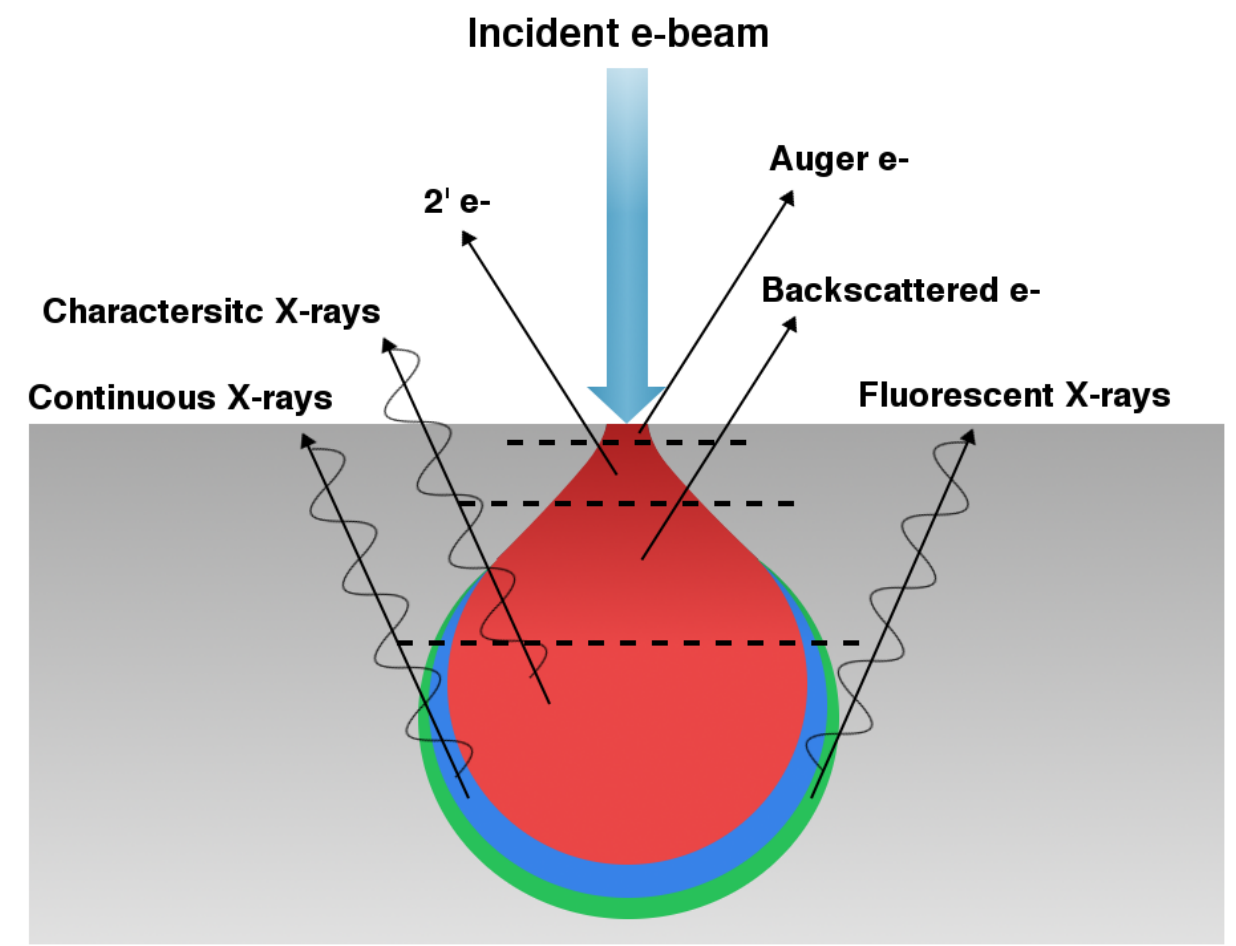

FIGURE 2.11 The interaction volume of an incident beam of electrons produces a variety of effects at different depths. SEM utilizes the secondary $2^{\prime}$ electrons for surface imaging whereas EDS utilizes characteristic x-rays for elemental analysis

bear no significant dependence upon the composition of the region of their origin, they are highly sensitive to topographical features [130]. Consequently, these electrons are used to generate the traditional tomographical SEM images, which have become so prevalent in scientific literature, and are widely used in observing and characterizing thin film surface homogeneity [131]. The lateral resolution of such images is largely dependent upon the incident beam diameter, which may be as small as $10 \AA$, yielding surface tomographical images with resolutions well beyond the optical diffraction limit. The typical resolution of standard SEM is included in Table 2.2, along with the resolutions, limitations, and sample requirements of other standard materials characterization methods. 
Additionally, although the properties of the electrons themselves are not related to sample composition, the likelihood of their generation is dependent upon the composition of the sample from which they originate, which produces a degree of contrast between surfaces of differing composition, such as a metal film and its glass substrate. Moreover, by increasing the tilt angle of the sample, it is possible to increase secondary electron generation and thereby increase the contrast. Images produced that demonstrate a discernible difference between the two surfaces are then analyzed to produce an estimation of the film thickness.

SEM-EDS, on the other hand, relies on X-rays produced through the interaction of the incident electron beam upon the sample surface [132]. The high energy electrons bombard the sample and eject secondary electrons from the atomic orbitals. The ejection of said electrons leaves vacancies in the electron shell, which are filled by electrons in higher shells. When an electron drops down to fill the vacancy, an X-ray is produced, whose energy and wavelength are characteristic of the orbital from which it was generated, and therefore are characteristic of the atom as well. However, not all X-rays produced through this method are characteristic of the elements they are generated by. The incident electrons can interact with any electrons in the atomic orbitals, which can generate a spectrum of other X-rays of differing energies [105]. Nevertheless, the characteristic X-rays may be used to perform chemical analysis of the sample surface, which may be compared to the chemical composition of the substrate to determine the relative amount of each element in the film. Although not an absolute measure of elemental concentration, ratios of the relative amounts of the elements of interest may be generated to infer film characteristics or to compare the 
sample to other similar samples for differentiation purposes [133, 134].

\subsubsection{E-Beam Techniques: Method}

The electron beam may be generated through one of two different means: field emission or thermionic emission. In the former method, electrons are generated by placing a pointed cold cathode filament in a high potential electric field, which rips electrons away from the filament and directs them toward the sample. In the latter method, a strong current is passed through a conducting filament, which generates a beam of electrons that are directed toward the sample surface. However, the method of generation is a design parameter of the equipment, rather than an analytical variable. That being said, regardless of the method of generation, the electron beam must be created under a high vacuum in order to collimate the beam and prevent premature electron scattering.

The beam is directed toward the sample through a column of electromagnetic "lenses", which collimate, condense, and focus the beam upon the sample surface, as shown in Fig. 2.12. In addition to these lenses, a pair of scanning coils deflects the beam so that it may be raster scanned across the surface, so as to generate a twodimensional image or analyze a specific section of the sample. The SEM equipment may also incorporate detectors for each of the aforementioned phenomena; however, cross-sectional SEM generally utilizes only the secondary electron detector, whereas SEM-EDS uses both that and the X-ray detector for elemental analysis. In order to avoid artifacts caused by sample charging, operating the SEM at lower beam energies around $1.5 \mathrm{kV}$ or less can yield operating resolutions around $1 \mathrm{~nm}$, although 


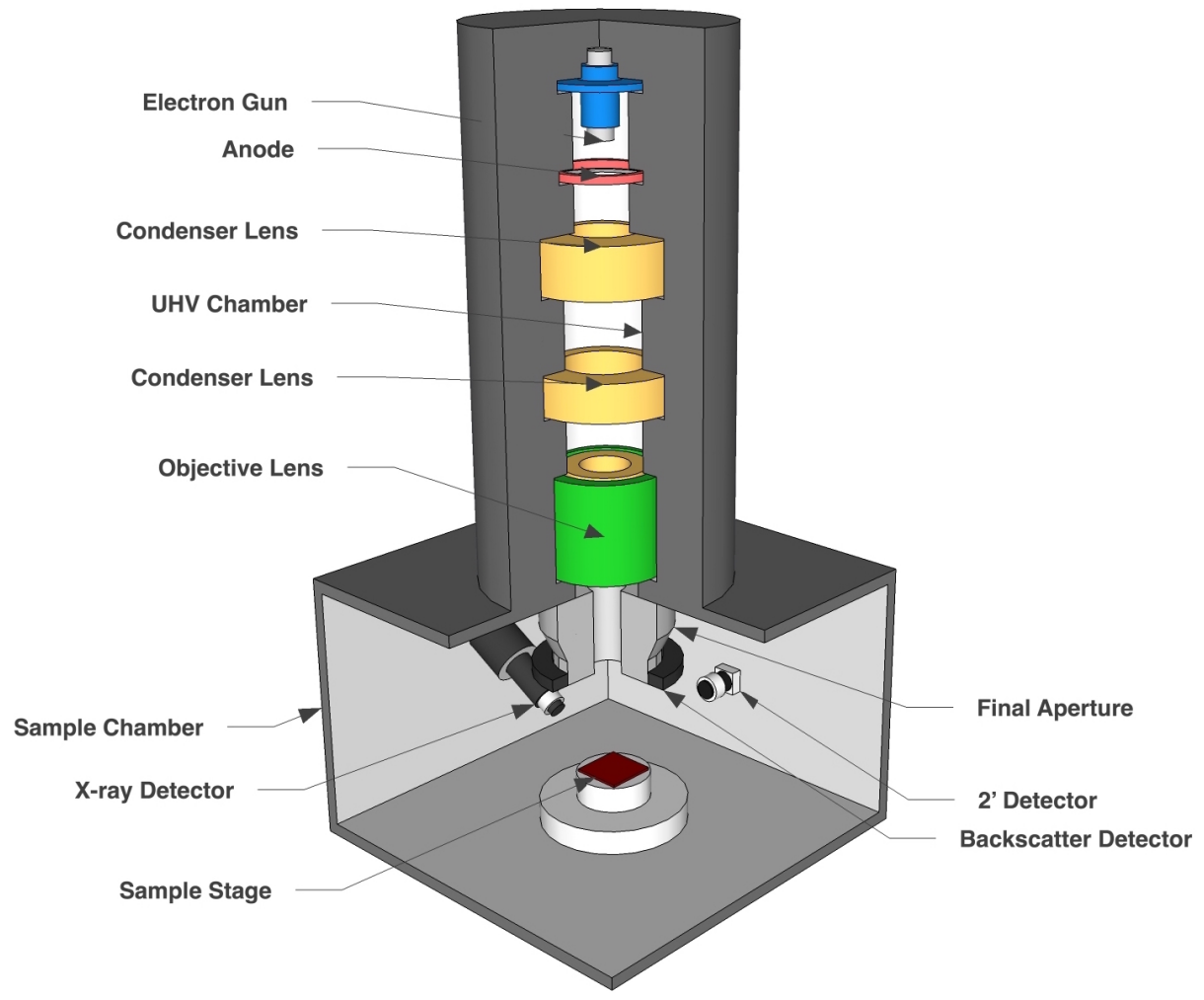

FIGURE 2.12 SEM systems are operated under ultra-high vacuum to minimize electron diffraction and allow the condenser "lenses" to collimate the electron beam. The sample chamber is equipped with as many different detectors as is necessary, but SEM imaging is performed using the secondary $2^{\prime}$ electron detector.

insulating samples like the glass or ceramic substrates used in optics should still be properly grounded [135].

Cross-sectional SEM analysis is functionally identical to traditional SEM in that a beam of electrons is directed toward the surface of a sample thin film-coated material. The principal difference is that the cross-sectional analysis necessitates that the region of interest be either the boarder of a thin film where the full thickness of the film atop the substrate can be observed, or else is a surface created by cutting the sample to produce such a cross-section. In the event that an observable border is present by design, the procedure is non-destructive in nature; however, should there 
be no such facet, this technique may necessitate damaging the sample.

By contrast, SEM-EDS focuses the incident e-beam onto a sample spot that is analyzed for a set amount of time, typically around $100 \mathrm{~s}$, so that the electrons can interact with each element of the sample and under interrogation. Although EDS can be achieved using any e-beam system, the combination of EDS with SEM allows for the secondary electron imaging to be utilized as a targeting system. The SEM generates a two dimensional image of an area of the sample, from which a point or series of points are selected for interrogation. Analysis of more points allows for statistical verification of film thickness, given that inhomogeneities may result in slight variations in thickness calculations.

\subsubsection{E-Beam Techniques: Cross-Sectional SEM Analysis}

With regard to thin film thickness measurement, cross-sectional SEM analysis is performed using image processing software, provided that an appropriate cross-section of the film and substrate layers has been recorded such that each layer is distinguishable from the others. Certain manufacturers include image processing as a tool incorporated into the equipment's operational software; however, there are also a number of third party applications that perform the same tasks, including ImageJ, Gimp, and Adobe Photoshop, assuming that a relevant scale has been included in the image. By comparing the height of the thin film in pixels over the length of the image to the scale provided by the SEM software, it is possible to calculate the thickness of the film in nanometers. The software may also need to take into account the magnification and the tilt angle of the mount in order to obtain an accurate reading, which may 


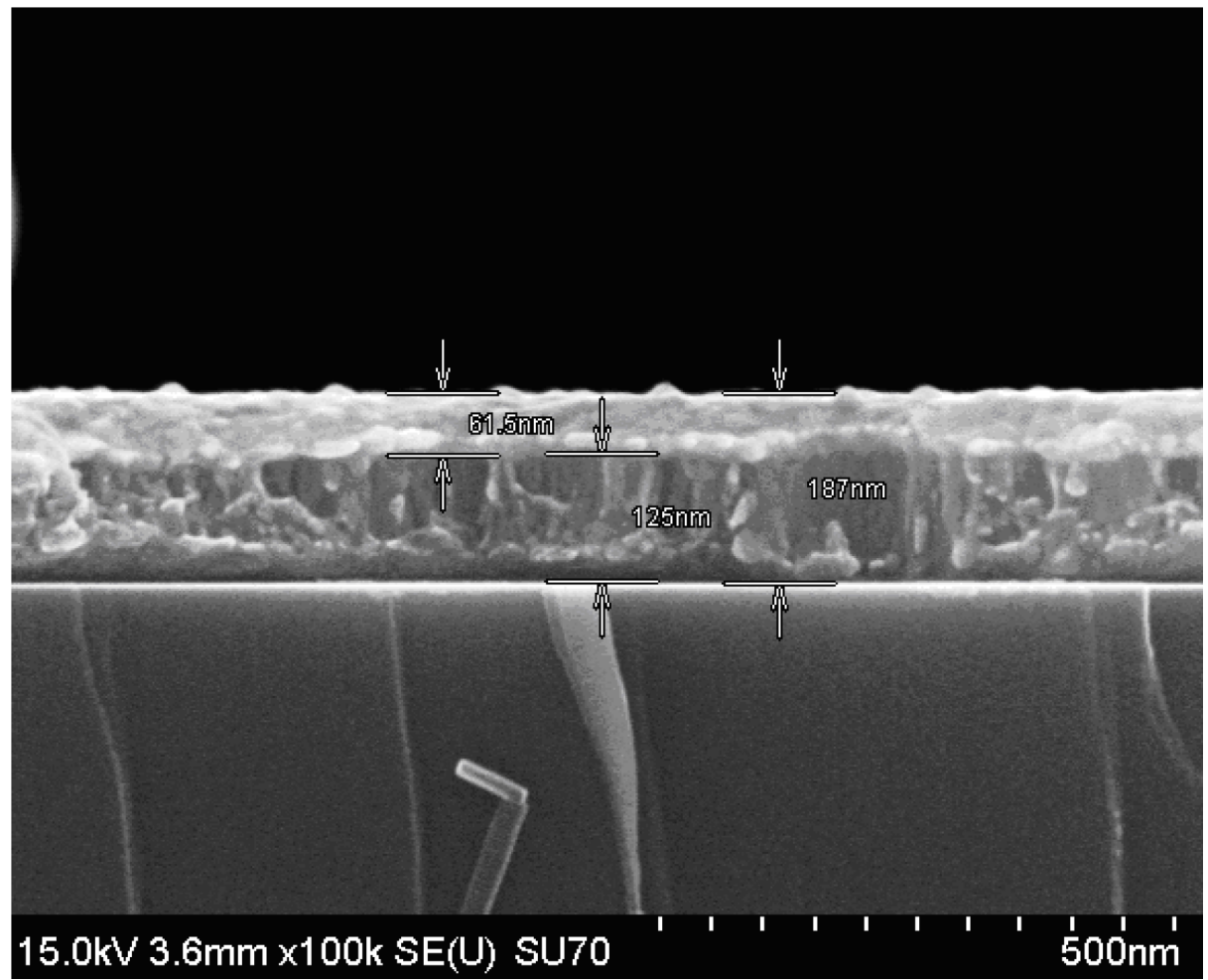

FIGURE 2.13 Software analysis of the SEM cross-section image reported in Sharp et al. reveals that the thickness of the $\mathrm{Au}$ film is $60.1 \mathrm{~nm}$ with a $123.5 \mathrm{~nm}$ thick Ni film underneath on a Si substrate, both with a claimed resolution less than $1 \mathrm{~nm}$; although that resolution may vary depending upon the degree of contrast between subsequent layers. [Reprinted from Microscopy and Microanalysis, 14, S. Sharp, et al. , "Thin film properties measurement using SEM-based energy dispersive spectroscopy for nanoscience studies", Page 337, Copyright (2008), with permission from Cambridge University Press]

compound error and result in detrimental effects on the resolution of the technique depending on the accuracy of the mounting angle measurement.

The SEM image shown in Fig. 2.13 demonstrates an example of a two-film system deposited on a silicon substrate [102]. The system under interrogation consists of a thin gold film deposited over a nickel film adhered to the surface of the silicon substrate, each of which is clearly distinguishable due to the distinct difference in contrast presented by each material. The image was analyzed using the LayerProbe 
software and yielded thicknesses of $60.1 \mathrm{~nm}$ for the gold film and $123.5 \mathrm{~nm}$ for the nickel film. The paper also reported that measurements had been recorded for the same system using X-Ray Fluorescence (XRF), yielding thickness values of $61 \mathrm{~nm}$ and $141 \mathrm{~nm}$ respectively. Moreover, the software claims that the resolution for each measurement is $0.7 \mathrm{~nm}$ for this analysis, although in other cases error was as low as $0.1 \mathrm{~nm}$; whereas the resolution of XRF measurements was only $25 \mathrm{~nm}$ and $24 \mathrm{~nm}$ respectively [102].

These values suggest that SEM image analysis can provide an extremely accurate measurement of film thickness; however, the situation presented is an ideal example wherein the films are easily distinguished and there does not appear to be any blatant image aberration. Under less-than-ideal situations, the measurement resolution is quickly compromised due to confounding factors such as image aberrations, distortion, lack of contrast, and charge build-up. Moreover, the facet chosen for analysis may not be representative of the entire surface, so obtaining an accurate thickness measurement may necessitate the destruction of the sample such that additional cross-sections may be imaged. Whereas other methods of analysis may nondestructively analyze different portions of the film, whether through raster-scanning the sample surface or independently changing the investigated area, cross-sectional SEM reveals only information about the observable face. In cases where the sample is inhomogeneously deposited, cross-sectional SEM may yield in inaccurate or misleading measurement. Consequently, SEM analysis is often used to visually present the film geometry and thereby gain an estimation of its thickness and surface roughness, but is typically used in conjunction with another analytical method for a more 


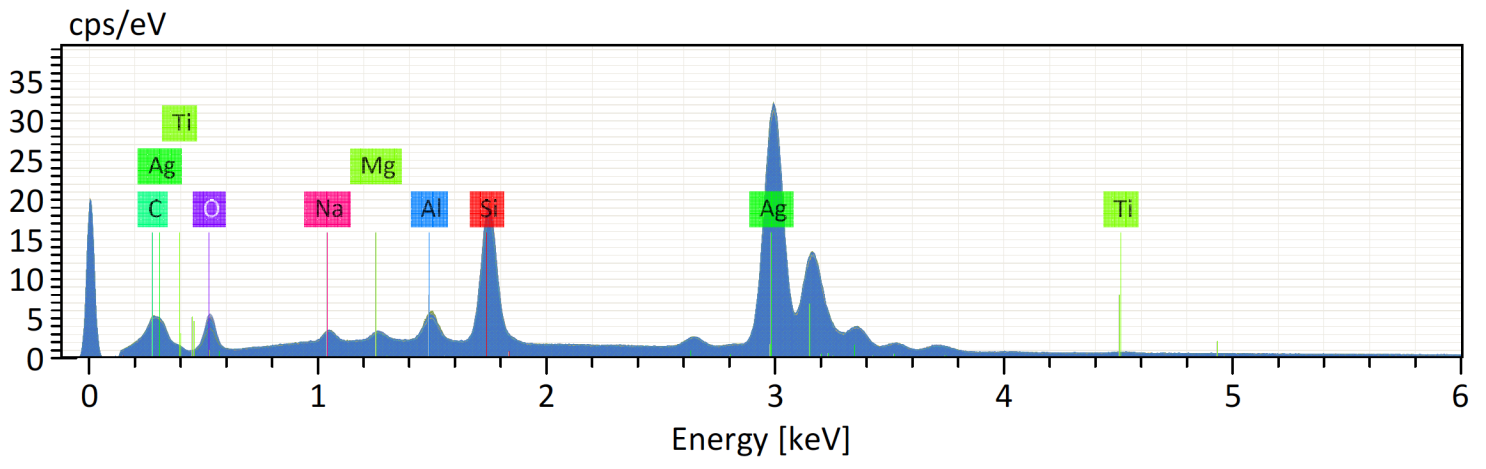

FIGURE 2.14 EDS analysis yields an X-ray spectrum, with characteristic peaks for each element present. Comparing peak ratios of reliable elements, like $\mathrm{Si}$, to the elements of the thin film, $\mathrm{Ag}$ and $\mathrm{Ti}$, it is possible to calculate film thickness using spectrum analyzer software developed at the National Institute of Standards and Technology (NIST).

accurate empirical measure.

\subsubsection{E-Beam Techniques: SEM-EDS Analysis}

In performing SEM-EDS analysis of a sample thin film deposited on a given substrate, the SEM imaging system is utilized as a targeting system so that the regions of interest can be identified by the user and interrogated by the e-beam. Each element yields characteristic X-rays resultant from electron interactions with electrons in the core atomic orbitals, allowing for the recording of an X-ray energy spectrum, which depicts the relative presence of each element based on the amplitude of recorded X-rays by their characteristic energy. In order to accurately record ultra-thin films of elements with high characteristic X-ray energies, a high speed silicon drift detector is necessary, due to the need to process counts at a sufficient rate to record the shorter wavelength X-rays.

Chininis et al. utilized an SEM equipped with a Bruker Quantax 200 Silicon Drift Detector with Xflash6 to interrogate samples of titanium and silver deposited 
on silicon glass substrates in order to determine the respective deposition rates of the two metals in a cold sputter vacuum deposition system [83]. The X-ray energy spectrum recorded for each sample was utilized to compare the peak amplitude of the silicon peak to that of the element being analyzed, as shown in Fig. 2.14. Silicon was used due to its stability within the glass chemistry, allowing for the recording of reliable Counts Per Second by electron-volt (cps/eV) for each of the relevant elements in the sample.

Following that, elemental ratios of silicon to the element of interest (either silver or titanium) were generated and the NIST DTSA-II (National Institute of Standards and Technology Desktop Spectrum Analyzer-II) software was used to produce a simulation of experimental conditions, while iteratively varying film thickness on a homogenous substrate in order to compare the recorded elemental ratios to those predicted though simulations. The ratios of silicon/titanium and silicon/silver were calculated for each simulation, varying film thickness until the ratios matched the recorded data, allowing for the determination of film thickness by comparing recorded measurements to the theoretically predicted models. The authors were able to calculate thickness measurements in such a manner and determine the titanium deposition rate to be $0.10 \mathrm{~nm} / \mathrm{s}$ and the silver deposition rate to be $1.19 \mathrm{~nm} / \mathrm{s}$. Consequently, provided the SEM is equipped with an appropriate X-ray detector, it may be possible to perform calculations to determine the film thickness by comparing measured values to DSTA software simulations based on NIST data. 


\subsection{Characterization limitations \& considerations}

The techniques discussed herein are some of the more useful methods of characterizing metal thin films. However, there are myriad other modalities for characterizing thin films, many of which are listed in Table 2.2. Each of these technologies has its benefits, but many are poorly suited to characterizing metal thin films in particular. Some of the more common limitations are included in the sections to follow, along with some useful considerations for the interested reader.

\subsubsection{Radiative absorption}

Many of the techniques mentioned herein and included in Table 2.2 are based on some form of photon propagation, whether within the X-ray, UV, Visible, or Infrared spectra. Consequently, these methods are subject to the influence of the optical properties of the samples in question, such as their absorption and scattering coefficients. This work focuses on metal films, which samples are largely reflective, particularly at greater thicknesses; however, they also tend to have a non-zero absorption coefficient, which is incorporated as part of the complex refractive index $\tilde{n}=n+i \kappa$, where $n$ is the real portion of the refractive index, which governs the relative speed of light in the medium, and $\kappa$ is the imaginary component, which governs optical absorption.

The complex refractive index, and therefore the absorption of photons, may have a profoundly negative effect on the efficacy of any radiative technique, particularly techniques like XRR and ellipsometry, which rely heavily upon specific changes resultant from the incident beam partially reflecting off the front and rear faces of 
the sample at each film interface. The greater the absorption within the film, the less accurate the measured change will be. This is particularly true for thicker films, wherein significant absorption reduces the effective mean free path of the photons passing through the film, which consequently decreases the number of photons that are able to escape the medium and therefore the intensity of the detected beam. This effect may alter the amplitude of the reflected intensity profile and skew the data away from the true values of film thickness, which can have a compounding effect for techniques that rely on computational curve fitting for thin film analysis. It is for this reason that many thin film characterization companies state that their software is only reliable for metal films between $0.5-50 \mathrm{~nm}$ thick, beyond which regimes the software can no longer accurately match the model to the data in a reliable fashion; although custom-built laboratory equipment may be able to out-perform commercially available equipment. Figure 2.5 is a principal example of such limitations, as the data for the thicker films is less defined and resulted in a poorer parameterized fit. The margin of error in the fit resulted in a slightly worsened resolution as well. It is advisable, therefore, that the radiative absorption spectrum of the elements that comprise the sample be considered when selecting a radiative technique, given that metals may be highly absorbing in the one spectral regime, but largely transmissive in another.

\subsubsection{Sample conductivity}

Another significant category of techniques utilized in thin film characterization involves the use of electron beams, as are used in SEM and Auger Electron 
Spectroscopy (AES), beams of charged ions, as used in Medium Energy Ion Scattering (MEIS), or electrical current, as employed in Scanning Tunneling Microscopy (STM). As a result, all such techniques are subject to the likelihood for charge buildup on the sample surface if not appropriately grounded, which can have substantial consequences for both the resolution of the technique and the serviceability of the equipment.

This work focuses on metal films in optical applications, which frequently involve film deposition onto optically transparent, but electrically insulating substrates. That being the case, electrical charge build-up is a strong possibility. Techniques like SEM, which rely on the detection of electrons ejected from the sample utilize incredibly sensitive equipment designed to detect small amounts of electrons. Consequently, an charge build-up may permanently damage the detector/analyzer, but will almost certainly "white-wash" the SEM image, thereby disrupting the necessary contrast for discerning film layers, deteriorating the spatial resolution of the image, and rendering any desired tomographical profile unreliable. Operating at a lower vacuum and reintroducing gases into the SEM may improve contrast and allow for charge to be conducted away from the sample surface, but also introduces a greater likelihood for e-beam interaction with the gas in the form of diffraction or scattering, which can have a negative influence on spatial resolution.

Moreover, techniques that employ X-ray radiation may also suffer from chargerelated problems as X-rays of such a wavelength as to be capable of interacting with the valence electrons in metal films and thereby elevating them to a higher energy state or otherwise ejecting them away from the film surface entirely. In the case of 
XRR, the charge build-up may damage the equipment as it discharges to other components, but may also affect the intensity of the specularly reflected beam. The use of conductive adhesive, such as copper tape, to form an electrical connection between the film layer and the sample stage may mitigate charge build-up concerns, but may have undesirable consequences on the adhesion regions of the sample, undermining otherwise non-destructive techniques.

\subsubsection{Derived vs. measured thickness}

Although Table 2.2 lists a number of other limitations and considerations, perhaps the most important consideration to bear in mind is the difference between computationally derived film thickness values, such as are provided by techniques like UV-Vis Spectrophotometry, XRR, and Ellipsometry, and thickness values directly correlated to an empirical measurement, as are provided by techniques like STM and AFM. The latter variety are typically contact-based technologies that often offer subnanometer depth resolutions and extremely reliable measurements owing to the use of vibration-damping systems to mitigate noise and error; however, they also necessitate transition regions on the samples to provide vertical contrast between substrate and film layers, and as such are limited either to only those samples that incorporate such regions, or those samples that allow for destructive testing conditions.

When such conditions are not met, the former variety of techniques are preferable, typically guaranteeing non-destructive testing and nano-scale resolutions. However, such methods rely on computational modeling or curve fitting to derive film characteristics, and as such are only as accurate as the margin of error for the mod- 
els. Consequently, the parameters of the model, such as refractive index and film thickness, may place limitations on the reliability of the measurement, as the error residuals increase. Figure 2.5 again provides an example wherein the analysis of thicker films exhibited greater error in the model fit and a correspondingly worsened depth resolution. As a result, derived thickness values are subject to computational error, inaccuracies of fit, and potentially substantial deviations away from the theoretically expected model. Moreover, when making use of any such computational techniques, it is essential that the operator also be aware of the theoretical assumptions upon which the software is operating. Moreover, said assumptions will likely change based on the various types of models and statistical analyses available in the software.

\subsection{Conclusions and developing technologies}

Due to their reflectivity and optical absorption, metal thin films often provide a challenge for many of the most predominant characterization techniques available. Of the methods covered in this review, AFM provides the most accurate and reliable measure of film thickness, with a potentially sub-Angstrom resolution, although it provides little other information and places certain requirements on the samples. Following that, SEM/EDS offers both an image of the film surface, which may grant information about the surface homogeneity, along with a computationally derived film thickness and elemental composition spectrum. The resolution is not quite on par with AFM, but for the purposes of film analysis in optics and biophotonics, it is sufficient, given that the technique can analyze films regardless of their optical reflectivity or 
absorption while still offering an optically relevant thickness measurement.

On the other hand, the other techniques, both based on radiative measurements, demonstrate varying efficacy and are highly dependent upon the film thickness, absorption, and refractive index. Ellipsometry is reportedly limited to metal films less than $50 \mathrm{~nm}$ thick, whereas XRR can reliably double that limit, although the degree of error in thickness value grows as thickness increases. In contrast to the other techniques, cross-sectional SEM does not actually provide a thickness value. However, image analysis can afford the observer with an idea of the overall thickness and the homogeneity of the film over a broad surface area. That said, it only provides local thickness characteristics at the cross section, and therefore would necessitate that the sample be fractured into multiple pieces to generate more cross-sections to analyze for statistical reliability.

Consequently, it is the opinion of the authors that if the refractive index of the metal film is of little to no consequence, contact based profilometry techniques like AFM offer the most reliable measurement of thickness alone. In the event that a transition region is not incorporated nor destructive testing methods permissible, SEM/EDS is a suitable alternative technique, if available. Nevertheless, each of the other techniques, particularly XRR, can provide reasonably accurate estimations through their derivations; however, investigators should be aware of the operational limitations of the techniques. It is recommended that should AFM or SEM/EDS not be a viable option, a reliable measure of thickness may be obtained through the combination of a radiative technique with cross-sectional SEM. Moreover, should the optical properties of the film be unknown and desired, employing additional thickness 
characterization techniques as confirmation can add reliability to radiative methods whose derivations may depend on geometric and optical parameters.

Having introduced some of the more predominant characterization methods and included a table of potentially viable alternatives that have seen some use in literature, it is worth noting that there are also new techniques in development for the characterization of thin films. One such technique, Total Internal Reflection Photoacoustic Spectroscopy (TIRPAS), falls into the category of Evanescent Field-based Photoacoustics (EFPA), and has been demonstrated in the characterization of the thickness and refractive index of optically transparent thin films [136]. Variations of the same technology have been suggested for use with metal and other optically absorptive thin films, in the form of Surface Plasmon Resonance Photoacoustic Spectroscopy (SPRPAS) and Optical Tunneling Photoacoustic Spectroscopy (OTPAS), in addition to applications involving the detection of absorbing particles [106, 137].

EFPA techniques utilize the same evanescent field depicted in Fig. 2.2, but with the intention of generating acoustic pulses following the absorption of the field's optical energy. In such applications, a sufficiently short laser pulse (less than $1 \mu \mathrm{s}$ ) totally internally reflects within a prism or waveguide, thereby generating an evanescent field within the external medium, which is typically the sample thin film. The portion of the field that penetrates through the film may be absorbed by chromophores in the medium beyond the film, which in turn incites an ultrasonic pressure wave through the optical absorption and resultant rapid thermoelastic expansion of the absorber. This acoustic energy may then be detected by a suitable ultrasonic transducer, which yields information about the film's optical and geometric properties, including re- 
fractive index and thickness [138]. The peak-to-peak magnitude of the photoacoustic pressure wave is directly related to the film thickness, its refractive index, and the incident angle of the laser beam. Consequently, by scanning through a range of incident angles, it is possible to determine the critical angle of the film-prism interface, calculate the refractive index using Snell's Law based on the known refractive index of the prism, and thereafter derive the film's thickness. Although this method has similar limitations to the other aforementioned optical approaches, the range of detectable film thicknesses is theoretically directly related to the wavelength of the incident light. Consequently, for thicker films, a longer wavelength should ideally provide accurate results, whereas for thinner films, a shorter wavelength may prove more effective.

Nevertheless, considering the recent breakthroughs in thin film chemistry, film deposition techniques, and meta-material fabrication, along with the continuous stream of new optical thin film applications being envisioned each year, the accurate depth profiling of thin films on insulating substrates represents a critical milestone in the advancement optical technologies. This is particularly the case for technologies akin to the waveguides discussed in Section 2.1.1, which rely on sub-wavelength electromagnetic effects, such as evanescent field interactions and surface plasmon resonance, to achieve what macroscale techniques could not. As such, whether it is a new conceptual modality still being substantiated or an established technique being iteratively improved, the further advancement of both noted and novel thin film characterization technologies is anticipated to play a significant role in the future of optics and biophotonics. 


\section{Chapter 3}

\section{Selective release waveguides and contact-based laser irradiation of tissue}

Portions of this chapter have been reproduced with permission from the Master's

Thesis entitled Photonic Ablation via Quantum Tunneling, authored by Paul J.D. Whiteside, Bioengineering Department, University of Missouri, May (2015) [1].

\subsection{Introduction}

Whereas the previous chapters first presented a background in medical biophotonics and the applications of light-based procedures therein followed by a comprehensive overview of metal thin film applications and the most appropriate and effective characterization techniques, the following sections discuss the concept of the planar "selective-release waveguide" (SRW), the principal innovation that forms the foundation of this dissertation and permits the incorporation of secondary technologies without necessitating their alteration or compromising their efficacy. As a brief overview, the SRW technique involves the fabrication of a planar optical waveguide through which laser light may be transmitted. Such waveguides are designed to transmit light through designated "active areas" on one of their largest facets. In 
biophotonics applications, upon direct contact of the waveguide's active area with a tissue or tissue-like medium, the light within the SRW substrate either refracts or optically tunnels out of the waveguide into the external medium. Furthermore, by tuning the composition and thickness of the active area materials, it is possible to regulate the portion of light that is transmitted out of the waveguide at each internal reflection point. The following sections discuss the technical aspects of this transmission approach in greater detail, along with a subsequent description of the experimental methods employed to establish the technique and quantify the transmission of pulsed light into ex vivo porcine tissue samples using planar, metal-clad SRW's of differing active area film thicknesses.

\subsection{Technical discussion}

The cardinal strength of the selective release waveguide technology lies in its foundation upon well-established optical principles, the most fundamental of which is the concept of the optical waveguide (a.k.a. "lightguide"). Such materials are utilized to transmit or redirect electromagnetic waves in the Ultraviolet (UV), Visible, and Near-Infrared (N-IR) spectra. Waveguides composed of optically transparent materials function by reflecting light within their bounding surfaces, forcing the light to propagate along their length $[92,139]$. This section provides a mathematical description for waveguide operation starting with optical first principles established by the likes of Isaac Newton, James Clerk Maxwell, and Augustin-Jean Fresnel. 


\subsubsection{Operating conditions of planar optical waveguides}

Optical waveguides have been used extensively in a variety of applications, including optoelectronic integrated circuitry, attenuated total reflection spectroscopy (ATR), and laser beam generation within planar waveguides; however, our application is intended for use in the controlled delivery of light into tissue and for that endeavor we must consider the types of interactions light can undergo along the length of the waveguides in question [69-71].

\subsubsection{Maxwell's and Fresnel's Equations}

With regard to the operation of optical waveguides, when considering the propagation of light within the waveguide substrate, the optical properties of both the substrate and cladding materials collectively determine the behavior of any beam of photons incident upon their interface. Such a beam may either refract into the external medium, travel along the interface, or totally internally reflect back into the substrate, dependent upon the relative refractive indices of the two media and the angle of the incident beam with respect to the interface. Waveguide operation demands that the light within the substrate repeatedly totally internally reflect at each interface, until reaching a designated exit aperture. The mathematical equations that govern each of these conditions are collectively known as the Fresnel Equations, and can be directly 
derived from Maxwell's Equations in isotropic media, shown below [140].

$$
\begin{array}{r}
\nabla \times \boldsymbol{H}=j+\frac{\partial D}{\partial t} \\
\nabla \times \boldsymbol{E}=-\frac{\partial B}{\partial t} \\
\nabla \cdot D=\rho \\
\nabla \cdot B=0 \\
j=\sigma \boldsymbol{E} \\
D=\varepsilon \boldsymbol{E} \\
B=\mu \boldsymbol{H}
\end{array}
$$

Expressing the Wave Equation in terms of only the Electric Field, $\boldsymbol{E}$, necessitates that the magnetic components of Maxwell's Equations $(\boldsymbol{H}$ and $B)$ be replaced with their electric equivalents. Combining and reducing Maxwell's Equations to only their electric components yields the Laplacian of $\boldsymbol{E}$, shown below in Eq. (3.8).

$$
\nabla^{2} \boldsymbol{E}=\mu \sigma \frac{\partial \boldsymbol{E}}{\partial t}+\mu \sigma \frac{\partial^{2} \boldsymbol{E}}{\partial t^{2}}
$$

One solution of this equation is in the complex form of a plane-polarized harmonic plane wave:

$$
\begin{aligned}
\boldsymbol{E} & =\mathcal{E} \exp \left[\mathrm{i} \omega\left(t-\frac{x}{v}\right)\right] \\
\text { with the condition that: } \frac{\omega^{2}}{v^{2}} & =\omega^{2} \varepsilon \mu-\mathrm{i} \omega \mu \sigma
\end{aligned}
$$


In this representation, $\mathcal{E}$ represents the vector amplitude for the Electric wave. The complex representation of the refractive index $(\widetilde{n}=n-\mathrm{i} \kappa)$ can then be readily derived from Eq. (3.10) by defining $\widetilde{n}^{2}=\frac{c^{2}}{v^{2}}$. This representation utilizes $n$ and $\kappa$ to represent the real and complex components of the refractive index respectively. By accounting for the speed of light $(c)$, relative permeability $\left(\mu_{r}\right)$, relative permittivity $\left(\varepsilon_{r}\right)$, and the complex refractive index, Equation (3.9) can be rewritten to incorporate some of the more useful optical properties. The equation can then be generalized for any plane-polarized wave propagating in the direction given by the unit vector $\hat{s}=$ $\langle\alpha i+\beta j+\gamma k\rangle$, to yield the Wave Equation in terms of $\boldsymbol{E}$ in Eq. (3.11). Additionally, a more rigorous derivation is included in Appendix A.

$$
\boldsymbol{E}=\mathcal{E} \exp \left[\mathrm{i}\left(\omega t-\left(\frac{2 \pi \widetilde{n}}{\lambda}\right)(\alpha x+\beta j+\gamma z)\right)\right]
$$

By a similar process, it is also possible to derive the Wave Equation in terms of only its magnetic components, yielding Eq. (3.12) below.

$$
\boldsymbol{H}=\mathcal{H} \exp \left[\mathrm{i}\left(\omega t-\left(\frac{2 \pi \widetilde{n}}{\lambda}\right)(\alpha x+\beta j+\gamma z)\right)\right]
$$

Whereas polarization does not have any implications for a beam that is normal to the interface, since the magnetic and electric components would both be parallel to the interface, in cases of oblique incidence in non-absorbing media, as is observed in optical waveguides, the polarization state of the beam has distinct implications with regard to the relative reflection and transmission coefficients. For the purposes 
(a)

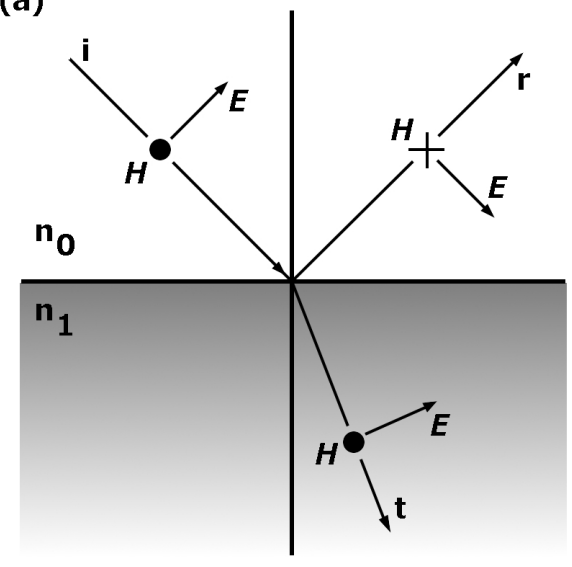

(b)

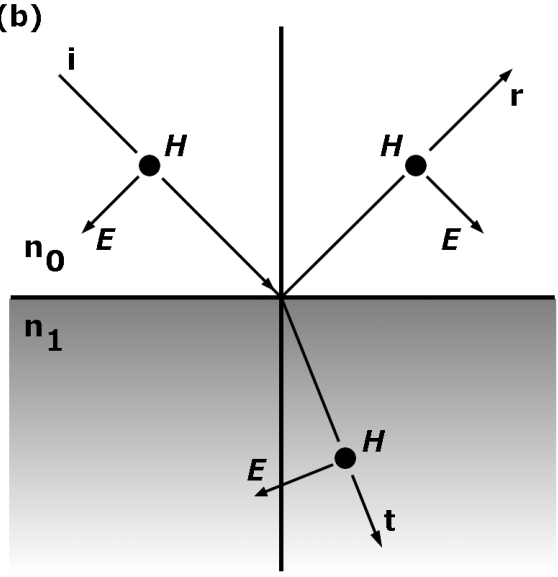

FIgURE 3.1 The sign conventions defining the positive directions for the Electric and Magnetic components of an obliquely incident beam upon a secondary medium for [left] Transverse Magnetic (TM, p-) polarized light and [right] Transverse Electric (TE, s-) polarized light.

of this study, we will consider the case of a beam that is linearly polarized with a transversely electric component. This polarization state is referred to as TE or spolarized, wherein "s" stands for "senkrecht" (German for "perpendicular"). The alternative polarization state is that of the transverse magnetic beam, referred to as TM or p-polarized, wherein "p" stands for "parallel" (the derivation for the transverse magnetic component is included in Appendix A).

The positive sign conventions for both polarizations of an obliquely incident beam are shown in Figure 3.1, with the conventions for TM polarized light on the [left] whereas those for TE polarized light are on the [right]. Based on these conventions, the boundary conditions for an obliquely incident s-polarized beam are mathematically represented below: 


$$
\begin{gathered}
\mathcal{E}_{i}+\mathcal{E}_{r}=\mathcal{E}_{t} \\
\mathcal{H}_{i} \cos \vartheta_{0}-\mathcal{H}_{r} \cos \vartheta_{0}=\mathcal{H}_{t} \cos \vartheta_{1}
\end{gathered}
$$

Again, we will derive these conditions such that they deal with only the electric field. It is important to note that the $\cos \vartheta$ terms arise from translating $\hat{s}$ over to perpendicular incidence, so that $\boldsymbol{E}$ and $\boldsymbol{H}$ are tangential (parallel) to the surface. Furthermore, it is convenient to define new variables to represent components of the translated boundary conditions, as shown below:

$$
\begin{array}{ll}
\mathrm{E}_{i}=\mathcal{E}_{i} & \mathrm{H}_{i}=\mathcal{H}_{i} \cos \vartheta_{0}=\mathcal{y}_{0} \cos \vartheta_{0} \mathrm{E}_{i} \\
\mathrm{E}_{r}=\mathcal{E}_{r} & \mathrm{H}_{r}=\mathcal{y}_{0} \cos \vartheta_{0} \mathrm{E}_{r} \\
\mathrm{E}_{t}=\mathcal{E}_{t} & \mathrm{H}_{t}=\mathcal{y}_{1} \cos \vartheta_{1} \mathrm{E}_{t}
\end{array}
$$

Rewriting the boundary conditions in the terms shown above yields Eq. (3.15) and (3.16), from which we can obtain expressions for the amplitude reflection and transmission coefficients for p-polarized light, $\rho_{s}$ and $\tau_{s}$ in Equations (3.17) and (3.18) respectively, by defining $\rho_{s}=\frac{E_{r}}{E_{i}}$ and $\tau_{s}=\frac{E_{t}}{E_{i}}$. In this form, $\mathcal{y}$ represents the optical admittance in non-absorbing media equivalent to $\mathcal{Y}=n \mathcal{Y}=n \sqrt{\frac{\varepsilon_{0}}{\mu_{0}}}$, where $\mathcal{Y}$ is the characteristic optical admittance of free space. 


$$
\begin{aligned}
& \mathrm{H}_{i}-\mathrm{H}_{r}=\mathrm{H}_{t} \\
& \mathcal{Y}_{0} \cos \vartheta_{0} \mathrm{E}_{i}-\mathcal{y}_{0} \cos \vartheta_{0} \mathrm{E}_{r}=\mathcal{y}_{1} \cos \vartheta_{1} \mathrm{E}_{t} \\
& =\mathcal{y}_{1} \cos \vartheta_{1}\left(\mathrm{E}_{i}+\mathrm{E}_{r}\right) \\
& \mathrm{E}_{i}\left(\mathcal{y}_{0} \cos \vartheta_{0}-\mathcal{y}_{1} \cos \vartheta_{1}\right)=\mathrm{E}_{r}\left(\mathcal{y}_{0} \cos \vartheta_{0}+\mathcal{y}_{1} \cos \vartheta_{1}\right) \quad \text { (Distribute and factor) } \\
& \frac{\mathrm{E}_{r}}{\mathrm{E}_{i}}=\frac{\mathcal{y}_{0} \cos \vartheta_{0}-\mathcal{y}_{1} \cos \vartheta_{1}}{\mathcal{y} \cos \vartheta_{0}+\mathcal{y} \cos \vartheta_{1}} \quad \text { (Rearrange fractions) } \\
& \rho_{s}=\frac{\mathrm{E}_{r}}{\mathrm{E}_{i}} \\
& \therefore \rho_{s}=\frac{\mathcal{y}_{0} \cos \vartheta_{0}-\mathcal{y}_{1} \cos \vartheta_{1}}{\mathcal{y} \cos \vartheta_{0}+\mathcal{y} \cos \vartheta_{1}}
\end{aligned}
$$

$$
\mathcal{y}_{0} \cos \vartheta_{0} \mathrm{E}_{i}-\mathcal{y}_{0} \cos \vartheta_{0} \mathrm{E}_{r}=\mathcal{y}_{1} \cos \vartheta_{1} \mathrm{E}_{t}
$$

(Continued from above)

$$
\begin{aligned}
\mathcal{y}_{0} \cos \vartheta_{0} \mathrm{E}_{i}-\mathcal{y}_{0} \cos \vartheta_{0}\left(\mathrm{E}_{t}-\mathrm{E}_{i}\right) & =\mathcal{y}_{1} \cos \vartheta_{1} \mathrm{E}_{t} \\
\mathrm{E}_{i}\left(2 \mathcal{Y}_{0} \cos \vartheta_{0}\right) & =\mathrm{E}_{t}\left(\mathcal{y}_{0} \cos \vartheta_{0}+\mathcal{y}_{1} \cos \vartheta_{1}\right) \\
\frac{\mathrm{E}_{t}}{\mathrm{E}_{i}} & =\frac{2 \mathcal{Y}_{0} \cos \vartheta_{0}}{\mathcal{Y}_{0} \cos \vartheta_{0}+\mathcal{Y}_{1} \cos \vartheta_{1}} \\
\tau_{s} & =\frac{\mathrm{E}_{t}}{\mathrm{E}_{i}} \\
\therefore \tau_{s} & =\frac{2 \mathcal{Y}_{0} \cos \vartheta_{0}}{\mathcal{Y}_{0} \cos \vartheta_{0}+\mathcal{y}_{1} \cos \vartheta_{1}}
\end{aligned}
$$

Next we define the expressions for the Irradiance of each of the three beams of interest, wherein $I_{i}$ represents the Irradiance of the incident beam derived from the general expression for Irradiance, as shown below. 


$$
\begin{aligned}
\boldsymbol{I} & =\frac{1}{2} \mathbb{R}\left\{\boldsymbol{E} \times \boldsymbol{H}^{*}\right\} \\
I & =\mathbb{R}\left\{\frac{1}{2} \mathrm{E}(y \cos \vartheta \mathrm{E})^{*}\right\} \\
\therefore I_{i} & =\frac{1}{2} \mathcal{Y}_{0} \cos \vartheta_{0} \mathrm{E}_{i} \mathrm{E}_{i}^{*}
\end{aligned}
$$

Following along the same approach, we will now define $I_{r}$ and $I_{t}$ in terms of $I_{i}$ in order to derive the relative reflection and transmission coefficients for s-polarized light, $R_{s}$ and $T_{s}$ respectively.

$$
\begin{array}{rlr}
I_{r} & =\mathbb{R}\left\{\frac{1}{2} \mathrm{E}_{r}\left(\mathcal{y}_{0} \cos \vartheta_{0} \mathrm{E}_{r}\right)^{*}\right\} & \text { (Scalar Reflected Irrdiance) } \\
& =\frac{1}{2} \rho_{s} \mathrm{E}_{i}\left(\mathcal{y}_{0} \cos \vartheta_{0} \rho_{s} \mathrm{E}_{i}\right)^{*} & \left(\mathrm{E}_{r}=\rho_{s} \mathrm{E}_{i}\right) \\
& =\frac{1}{2} \rho_{s}^{2} \mathcal{y}_{0} \cos \vartheta_{0} \mathrm{E}_{i} \mathrm{E}_{i}^{*} & \text { (Reflect the format of } \left.I_{i}\right) \\
\therefore I_{r} & =\rho_{s}^{2} I_{i} & (3.21) \\
I_{t} & =\mathbb{R}\left\{\frac{1}{2} \mathrm{E}_{t}\left(\mathcal{y}_{1} \cos \vartheta_{1} \mathrm{E}_{t}\right)^{*}\right\} & \text { (Scalar Transmitted Irradiance) } \\
& =\frac{1}{2} \tau_{s} \mathrm{E}_{i}\left(\mathcal{y}_{1} \cos \vartheta_{1} \tau_{s} \mathrm{E}_{i}\right)^{*} \\
& =\frac{1}{2} \tau_{s}^{2} \frac{\mathcal{y}_{1} \cos \vartheta_{1}}{\mathcal{y}_{0} \cos \vartheta_{0}} \mathcal{Y}_{0} \cos \vartheta_{0} \mathrm{E}_{i} \mathrm{E}_{i}^{*} \\
\therefore I_{t} & =\frac{\mathcal{y}_{1} \cos \vartheta_{1}}{\mathcal{y}_{0} \cos \vartheta_{0}} \tau_{s}^{2} I_{i} & \text { (Reflect the format of } \left.I_{i}\right) \\
\left.\mathrm{E}_{i}\right)
\end{array}
$$

From here, considering the simple energy balance equation below allows for the direct derivation of $R_{s}$ and $T_{s}$. It is also worth noting that by translating $\hat{s}$ to perpendicular incidence and incorporating the $\cos \vartheta \operatorname{components,~the~traditional~}$ 
condition that $1-R=T$ still holds true.

$$
\begin{aligned}
& \boldsymbol{I}_{0}=\boldsymbol{I}_{1} \quad \text { (Conservation of energy) } \\
& \mathbb{R}\left\{\frac{1}{2} \boldsymbol{E}_{0} \times \boldsymbol{H}_{0}^{*}\right\}=\mathbb{R}\left\{\frac{1}{2} \boldsymbol{E}_{1} \times \boldsymbol{H}_{1}^{*}\right\} \quad \text { (Sub in. Eq. 3.19) } \\
& \mathbb{R}\left\{\frac{1}{2}\left(\mathrm{E}_{i}+\mathrm{E}_{r}\right)\left(\mathrm{H}_{i}-\mathrm{H}_{r}\right)^{*}\right\}=\mathbb{R}\left\{\frac{1}{2} \mathrm{E}_{t} \mathrm{H}_{t}^{*}\right\} \quad \text { (Scalar components) } \\
& \frac{1}{2}\left(\mathrm{E}_{i}+\mathrm{E}_{r}\right)\left(\mathcal{y}_{0} \cos \vartheta_{0} \mathrm{E}_{i}-\mathcal{y}_{0} \cos \vartheta_{0} \mathrm{E}_{r}\right)^{*}=\frac{1}{2} \mathrm{E}_{t} \mathcal{y}_{1} \cos \vartheta_{1} \mathrm{E}_{t}^{*} \quad(\mathrm{H}=\mathcal{y} \cos \vartheta \mathrm{E}) \\
& \frac{1}{2} \mathcal{Y}_{0} \cos \vartheta_{0}\left(\mathrm{E}_{i}+\rho_{s} \mathrm{E}_{i}\right)\left(\mathrm{E}_{i}-\rho_{s} \mathrm{E}_{i}\right)^{*}=\frac{1}{2} \mathcal{Y}_{1} \cos \vartheta_{1} \tau_{s}^{2} \mathrm{E}_{i} \mathrm{E}_{i}^{*} \quad\left(\mathrm{E}_{r}=\rho_{s} \mathrm{E}_{i}\right) \\
& \frac{1}{2} \mathcal{Y}_{0} \cos \vartheta_{0} \mathrm{E}_{i} \mathrm{E}_{i}^{*}\left(1-\rho_{s}^{2}\right)=\frac{1}{2} \mathcal{Y}_{1} \cos \vartheta_{1} \tau_{s}^{2} \mathrm{E}_{i} \mathrm{E}_{i}^{*} \quad \text { (Factor) } \\
& =\frac{1}{2} \frac{\mathcal{y}_{1} \cos \vartheta_{1}}{\mathcal{y}_{0} \cos \vartheta_{0}} \mathcal{Y}_{0} \cos \vartheta_{0} \tau_{s}^{2} \mathrm{E}_{i} \mathrm{E}_{i}^{*} \\
& I_{i}\left(1-\rho_{s}^{2}\right)=\frac{1}{2} \frac{\mathcal{y}_{1} \cos \vartheta_{1}}{\mathcal{y}_{0} \cos \vartheta_{0}} I_{i} \quad \text { (Sub. in Eq. 3.20) } \\
& I_{i}-\rho_{s}^{2} I_{i}=\frac{1}{2} \frac{\mathcal{y}_{1} \cos \vartheta_{1}}{\mathcal{Y}_{0} \cos \vartheta_{0}} I_{i} \quad \text { (Distribute) } \\
& I_{i}-I_{r}=I_{t} \quad \text { (Sub in Eq. 3.21, 3.22) } \\
& \left.1-\frac{I_{r}}{I_{i}}=\frac{I_{t}}{I_{i}} \quad \text { (Divide by } I_{i}\right) \\
& \therefore 1-R=T
\end{aligned}
$$

Following from this, we find the expressions for $R_{s}$ and $T_{s}$ shown below in Equations (3.24) and (3.25), respectively.

$$
\begin{array}{r}
R_{s}=\rho_{s}^{2}=\left(\frac{\mathcal{y}_{0} \cos \vartheta_{0}-\mathcal{y}_{1} \cos \vartheta_{1}}{\mathcal{y}_{0} \cos \vartheta_{0}+\mathcal{y}_{1} \cos \vartheta_{1}}\right)^{2} \\
T_{s}=\tau_{s}^{2}\left(\frac{\mathcal{y}_{1} \cos \vartheta_{1}}{\mathcal{y}_{0} \cos \vartheta_{0}}\right)=\frac{4 \mathcal{y}_{0} \mathcal{y}_{1} \cos \vartheta_{0} \cos \vartheta_{1}}{\left(\mathcal{y}_{0} \cos \vartheta_{0}+\mathcal{y}_{1} \cos \vartheta_{1}\right)^{2}}
\end{array}
$$

By substituting $\mathcal{Y}=n \mathcal{Y}$, the traditional form of the Fresnel equations for spolarized light are produced. 


$$
\begin{aligned}
R_{s} & =\left(\frac{n_{0} \cos \vartheta_{0}-n_{1} \cos \vartheta_{1}}{n_{0} \cos \vartheta_{0}+n_{1} \cos \vartheta_{1}}\right)^{2} \\
T_{s} & =\frac{4 n_{0} n_{1} \cos \vartheta_{0} \cos \vartheta_{1}}{\left(n_{0} \cos \vartheta_{0}+n_{1} \cos \vartheta_{1}\right)^{2}}
\end{aligned}
$$

These equations describe the degree of transmission and reflection observed at the intersections of two materials of differing optical properties, namely the waveguide and any surrounding material. When a beam of light is incident upon a planar interface, such as those shown in Figure 3.1, a portion of the light may reflect whereas the remainder may refract into the secondary medium. For each of the preceding equations, $T_{s}$ represents the percent of light incident upon the intersection that is transmitted into the second medium, whereas $R_{s}$ represents the percent of light reflected back within the original material. A similar derivation yields the percent reflection $\left(R_{p}\right)$ and transmission $\left(T_{p}\right)$ for p-polarized light, shown below in Equations (3.28) and (3.29) respectively.

$$
\begin{aligned}
& R_{p}=\left[\frac{\frac{n_{0}}{\cos \vartheta_{0}}-\frac{n_{1}}{\cos \vartheta_{1}}}{\frac{n_{0}}{\cos \vartheta_{0}}+\frac{n_{1}}{\cos \vartheta_{1}}}\right]^{2} \\
& T_{p}=\frac{\frac{4 n_{0} n_{1}}{\cos \vartheta_{0} \cos \vartheta_{1}}}{\left(\frac{n_{0}}{\cos \vartheta_{0}}+\frac{n_{1}}{\cos \vartheta_{1}}\right)^{2}}
\end{aligned}
$$

\subsubsection{Snell's Law}

In cases of refraction, the Fresnel Equations govern the degree of reflection and refraction at the interface, leaving a portion of the incident beam to propagate within 

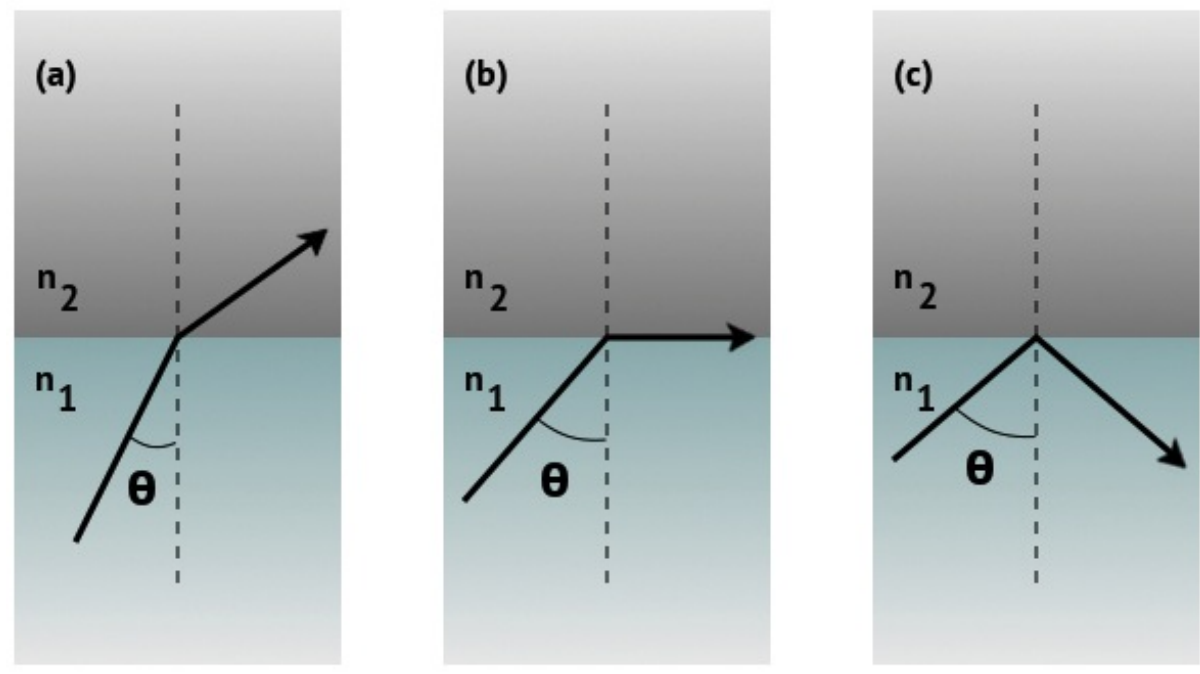

FiguRE 3.2 The conditions for the three possible outcomes of the Fresnel equations and Snell's Law are: (a) when $n_{2}>n_{1}$ light is refracted into the second material, (b) when $n_{2}<n_{1}$ and $\theta=\theta_{c}$ the light travels along the intersection, or (c) when $n_{2}<n_{1}$ and $\theta>\theta_{c}$ the light undergoes TIR.

each material. However, there are two additional possible outcomes, wherein there is no observable refraction and $T=0$, as shown in Figure $3.2(\mathbf{b})$ and (c). In order to determine the conditions at which these outcomes occur, we must first derive another equation to directly relate the refractive indices and propagation angles of each the incident, transmitted, and reflected beams.

We start by considering the Irradiance of each of the three beams individually. The general expression for Irradiance can be concisely represented in the following manner:

$$
I=\frac{1}{2} n \mathcal{Y}|\mathcal{E}|^{2} \exp \left[\frac{-4 \pi k}{\lambda}(\alpha x+\beta y+\gamma z)\right]
$$

Following that, we apply a few simple boundary conditions: 
- the tangential components of $\boldsymbol{E}$ and $\boldsymbol{H}$ are continuous across the boundary

- $\hat{s}_{\text {reflected }}=\left\langle\alpha_{r}, \beta_{r}, \gamma_{r}\right\rangle$

- $\hat{s}_{\text {transmitted }}=\left\langle\alpha_{t}, \beta_{t}, \gamma_{t}\right\rangle$

From these conditions, we can represent each of the three wavefronts of interest incident, reflected, and transmitted - consistently for both Electric and Magnetic components in the following forms:

$$
\begin{array}{r}
\text { Incident }: \exp \left[\mathrm{i}\left(\omega_{i} t-\left(\frac{2 \pi n_{1}}{\lambda_{i}}\right)\left(x \sin \vartheta_{1}+z \cos \vartheta_{1}\right)\right)\right] \\
\text { Reflected }: \exp \left[\mathrm{i}\left(\omega_{r} t-\left(\frac{2 \pi n_{1}}{\lambda_{r}}\right)\left(\alpha_{r} x+\beta_{r} y+\gamma_{r} z\right)\right)\right] \\
\text { Transmitted }: \exp \left[\mathrm{i}\left(\omega_{t} t-\left(\frac{2 \pi n_{2}}{\lambda_{t}}\right)\left(\alpha_{t} x+\beta_{t} y+\gamma_{t} z\right)\right)\right]
\end{array}
$$

Then, in order to satisfy continuity for all $x, y$, and $t$ at $z=0$, it can be determined that $\omega_{i} \equiv \omega_{r} \equiv \omega_{t}$. In other words, there is no change in frequency for reflected or transmitted waves as compared to the incident wave. That being the case, we can extend the identical equality to the wavelength, such that $\lambda_{i} \equiv \lambda_{r} \equiv \lambda_{t}$. Additionally, since the representation of the incident beam is oriented such that it is independent of $y$, the conclusion can be drawn that the directions of the reflected and transmitted waves are confined to the plane of incidence. The mathematical implications of these observations are as follows: 


$$
\begin{aligned}
0 & \equiv n_{1} \beta_{r} \equiv n_{2} \beta_{t} \\
\left(\frac{2 \pi n_{1}}{\lambda}\right)\left(x \sin \vartheta_{i}+z \cos \vartheta_{i}\right) & \equiv\left(\frac{2 \pi n_{1}}{\lambda}\right)\left(\alpha_{r} x+\gamma_{r} z\right) \\
& \equiv\left(\frac{2 \pi n_{2}}{\lambda}\right)\left(\alpha_{t} x+\gamma_{t} z\right) \\
\therefore \sin \vartheta & =\alpha \\
\therefore \cos \vartheta & =\gamma \\
n_{1} \sin \vartheta_{i} & \equiv n_{1} \alpha_{r} \equiv n_{2} \alpha_{t}
\end{aligned}
$$

Following from Eq. 3.31 and defining the angles of reflection and transmission to be $\vartheta_{r}$ and $\vartheta_{t}$ respectively, we find the following relation to be true.

$$
\begin{aligned}
n_{1} \sin \vartheta_{i} & \equiv n_{2} \alpha_{t} \\
& =n_{2} \sin \vartheta_{t}
\end{aligned}
$$

Redefining $\vartheta_{i}$ and $\vartheta_{t}$ to be $\vartheta_{1}$ and $\vartheta_{2}$ respectively, results in the traditional expression for Snell's Law shown below:

$$
n_{1} \sin \vartheta_{1}=n_{2} \sin \vartheta_{2}
$$

\subsubsection{Waveguide operation}

In order for a material to operate as a waveguide, the light must be totally internally reflected within the bounds of the material, such that all of the light incident upon the intersection of the substrate and external medium reflects back within the original 
medium, as shown in Figure 3.2 (c). Optical waveguides operate exclusively under this third condition, such that the refractive index of the external medium $\left(n_{2}\right)$ is less than that of the waveguide $\left(n_{1}\right)$, forcing the light to internally reflect between its surfaces as it propagates along the length of the waveguide. However, in order to achieve total internal reflection (TIR), the incident angle of the light upon the boundary must also be greater than the critical angle, $\theta_{c}$, which can be mathematically determined from Snell's Law (eq. 3.33) [93].

$$
\begin{aligned}
& n_{1} \sin \theta_{1}=n_{2} \sin \theta_{2} \\
& \theta_{c}=\arcsin \left(\frac{n_{2}}{n_{1}}\right)
\end{aligned}
$$

Assuming that the external material is of lower refractive index than the waveguide, it can be determined that $\sin \theta_{2} \rightarrow 1$, which reduces Snell's Law to equation (3.35), allowing for direct calculation of the critical angle when $n_{1}$ and $n_{2}$ are known. Using this value in conjunction with the Fresnel equations for reflectivity for TE and TM polarized light (Eq. (3.26) and 3.28 respectively) allows for the generation of a plot depicting polarization-dependent reflectivity versus internal reflection angle. For example, the reflectivity for a waveguide composed of BK7 glass $\left(n_{1}=1.519\right)$ considering the external material to be air $\left(n_{2}=1.0\right)$ is shown in Figure 3.3 [72]. The graph demonstrates that the reflectivity for TE polarized light is consistently greater than for TM polarized light, until the critical angle at $\theta_{c}=41.17^{\circ}$, after which the light undergoes total internal reflection. Additionally, the TM polarization reflectivity drops to 0 at the Brewster Angle, which is given by Equation (3.36), and is derived 


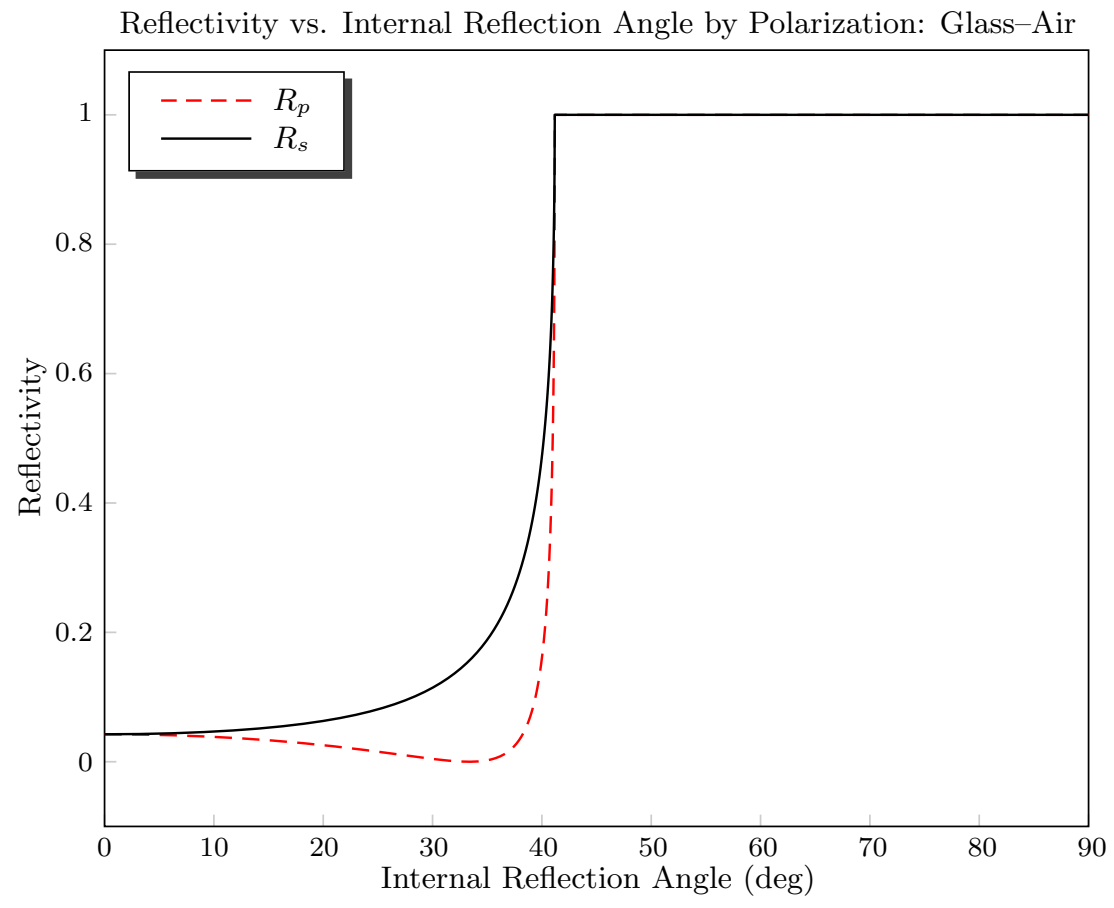

FIGURE 3.3 The reflectivity for p-polarized light is consistently lower than for s-polarized light, until the critical angle at $\theta_{c}=41.17^{\circ}$, after which the light undergoes TIR.

in Appendix (A.2.2.4).

$$
\theta_{B}=\tan ^{-1}\left(\frac{n_{2}}{n_{1}}\right)
$$

However, in most cases it can not be assumed that only air will come in contact with the waveguide. Consequently, for most waveguides TIR is ensured by design through the incorporation of a cladding layer of known refractive index to encapsulate the waveguide, as shown in Figure 3.4 [90, 141]. Typically, optical waveguide substrates are composed of transparent polymers, like Poly-methyl methacrylate (PMMA, $n=1.49)$, ceramics, or glasses, like Sapphire $(n=1.77)$ or Borosilicate BK7 glass $(n=1.519)[72,142]$. By selecting one such substrate with known refractive index, $\theta_{c}$ can subsequently be scaled by the appropriate selection of the cladding ma- 


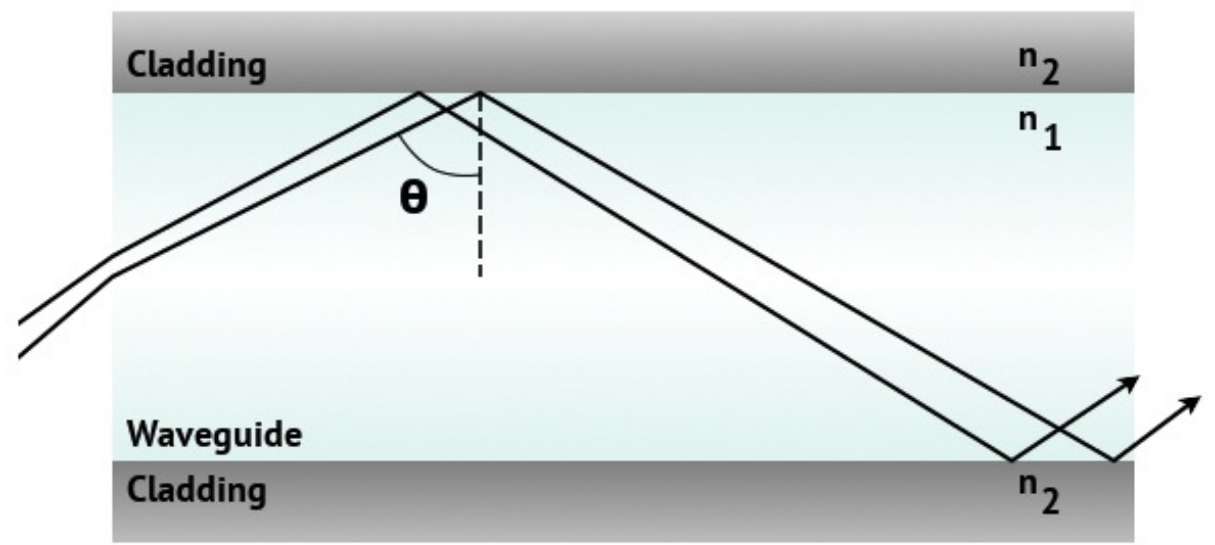

FIgURE 3.4 Light in a waveguide undergoes TIR when $n_{2}<n_{1}$ and $\theta$ exceeds the critical angle. This can be ensured by cladding the waveguide in a polymer or reflective metal layer of lower refractive index than the substrate.

terial. For example, using polymer claddings with refractive indices around $n=1.32$ on a BK7 glass substrate typically results in a relatively large $\theta_{c}=60.34^{\circ}$; however, choosing such a material limits the angles that support TIR to only those between $60.34^{\circ}$ and $90^{\circ}$.

On the other hand, certain metal claddings allow for a much broader range of total internal reflection angles, since they may have substantially lower refractive indices. For example, the polarization-dependent reflectivity plot for a BK7 glass waveguide clad in Silver $(n=0.142)$ is shown in Figure 3.5. The figure shows that the metal ensures a broad range of angles that support TIR after the critical angle of $5.36^{\circ}$, although the p-polarized light experiences a substantial decrease in reflectivity until the Brewster Angle at 5.34 . Such metal claddings demonstrate high reflectivity in the visible spectrum and support TIR throughout a much broader spectrum of angles; however, they also tend to introduce a degree of optical absorption, which may result in energy loss over long distances [73]. 


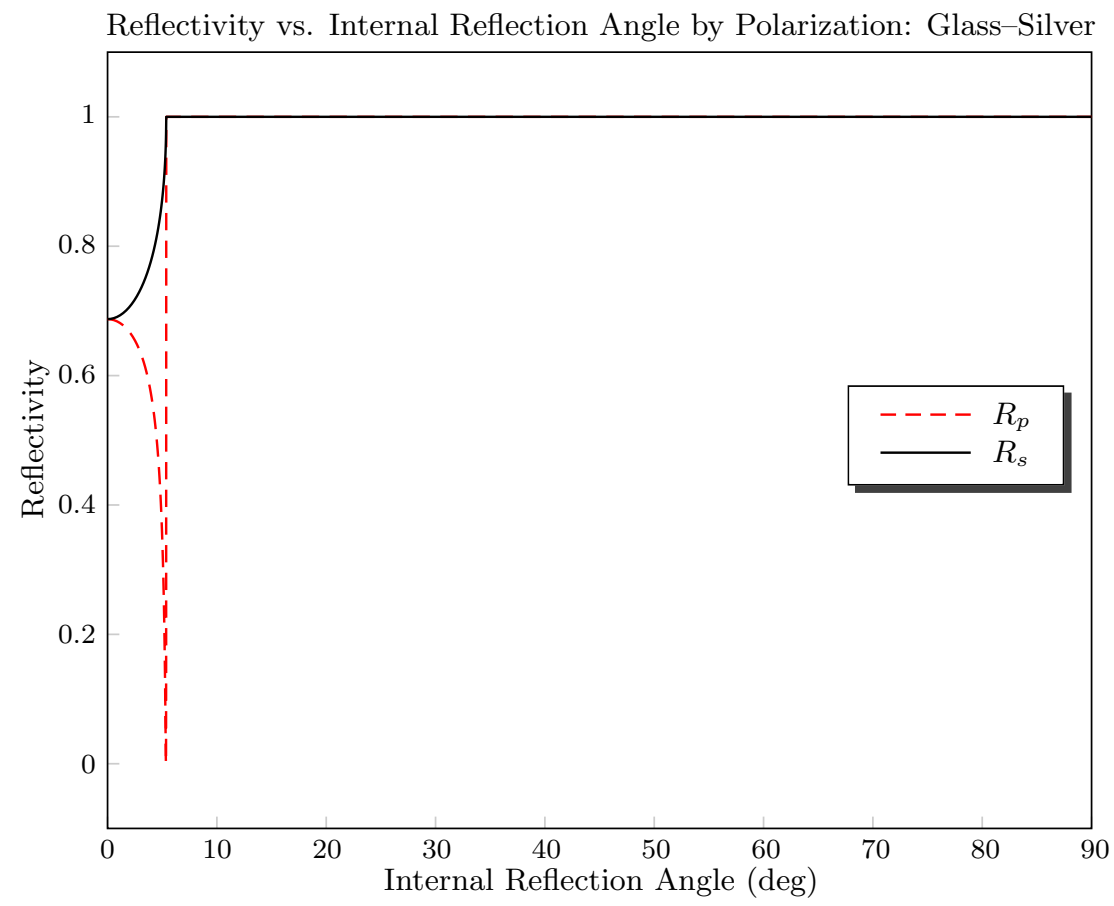

FigURE 3.5 Using Silver as a cladding allows for a wide range of acceptable angles that support TIR, from around $5.4<\theta_{1}<90^{\circ}$. However, the reflectivity of TM polarized light is severely limited at angles less than $\theta_{c}$.

\subsubsection{Evanescent fields and optical tunneling}

The following section provides a discussion of the nano-scale effects observed at the bounding interfaces of an optical waveguide. Whereas the previous explanation of TIR and waveguide operation provides a conceptual overview of the phenomena involved in light transmission, the wave nature of light causes a variety of problems when describing TIR, due to common assumptions resultant from classical mechanics.

In classical mechanics, particularly on the macroscale, light is traditionally treated as a particle propagating in distinct energy packets referred to as photons. As such, diagrams like that shown in Figure 3.4 depict the paths of these photons bouncing off the surfaces of the waveguide like particles off a barrier. However, research has 
demonstrated, even as far back as Isaac Newton, that the energy of the photons does not merely reflect off the surface, but rather exhibits a degree of penetration into the surrounding material [143].

\subsubsection{Evanescent fields}

By contrast, treating the light as an electromagnetic wave instead of a discrete particle reveals that upon interacting with a secondary material of lower refractive index, the energy of the incident wave slightly crosses the interface of the two materials, penetrating the external medium in the form of an exponentially decaying field of energy, referred to as the evanescent field. In mathematically deriving the existence of the evanescent field, it is necessary to account for the non-propagating nature of the field first by defining the "propagation" vector $\hat{s}_{2}=\left\langle\sin \theta_{2} i+0 j+\cos \theta_{2} k\right\rangle$, and second by recognizing that $\theta_{2}$ technically does not exist, since there is no refracting portion of the beam. Consequently, in the course of the derivation, all terms containing $\theta_{2}$ will need to be substituted out for terms that still apply to the situation of total internal reflection. We will also define $\boldsymbol{E}_{0,2}$ to be the electric field in the second medium and $\mathcal{E}_{0,2}$ to be the amplitude of the field of interest. It is also convenient to temporarily

define the wave vector $k_{2}=\frac{2 \pi \widetilde{n}}{\lambda_{0}}$ for simpler expressions during the derivation. 


$$
\begin{array}{rlr}
\boldsymbol{E}_{0,2} & =\mathcal{E}_{0,2} \exp \left[\mathrm{i}\left(\omega t-\left(\frac{2 \pi \tilde{n}}{\lambda_{0}}\right) \hat{s}_{2} \cdot \vec{r}\right)\right] & \text { (From Eq. A.15) } \\
& =\mathcal{E}_{0,2} \exp \left[\mathrm{i}\left(\omega t-k_{2} \hat{s}_{2} \cdot \vec{r}\right)\right] & \text { (Sub. in } \left.k_{2}\right) \\
& =\mathcal{E}_{0,2} \exp \left[\mathrm{i}\left(\omega t-k_{2} x \sin \theta_{2}-k_{2} z \cos \theta_{2}\right)\right] & \text { (Dot product) } \\
& =\mathcal{E}_{0,2} \exp \left[\mathrm{i}\left(\omega t-k_{2} x \sin \theta_{2} \pm k_{2} z\left(1-\sin ^{2} \theta_{2}\right)^{\frac{1}{2}}\right)\right] & \text { (Trig. substitution) } \\
& =\mathcal{E}_{0,2} \exp \left[\mathrm{i}\left(\omega t-k_{2} x \frac{n_{1}}{n_{2}} \sin \theta_{1} \pm k_{2} z\left(1-\frac{n_{1}^{2}}{n_{2}^{2}} \sin ^{2} \theta_{1}\right)^{\frac{1}{2}}\right)\right] & \quad \text { (Snell's law) } \\
& =\mathcal{E}_{0,2} \exp \left[\mathrm{i}\left(\omega t-k_{2} x \frac{n_{1}}{n_{2}} \sin \theta_{1} \pm \frac{k_{2}}{n_{2}} z\left(n_{2}^{2}-n_{1}^{2} \sin ^{2} \theta_{1}\right)^{\frac{1}{2}}\right)\right] \\
& \left.=\mathcal{E}_{0,2} e^{\mathrm{i}\left(\omega t-k_{2} x \frac{n_{1}}{n_{2}} \sin \theta_{1}\right)} e^{-\frac{k_{2}}{n_{2}} z\left(n_{1}^{2} \sin ^{2} \theta_{1}-n_{2}^{2}\right)^{\frac{1}{2}}} \quad \text { (Factor } n_{2}\right) \\
& =\mathcal{E}_{0,2} e^{\mathrm{i}\left(\omega t-\frac{2 \pi \tilde{n}_{2} n_{1}}{\lambda_{0} n_{2}} x \sin \theta_{1}\right)} e^{-\frac{2 \pi \tilde{n}_{2}}{\lambda_{0} n_{2}} z\left(n_{1}^{2} \sin ^{2} \theta_{1}-n_{2}^{2}\right)^{\frac{1}{2}}} \quad \text { (Factor i) } \\
& \left.=\mathcal{E}_{0,2} e^{\mathrm{i}\left(\omega t-\frac{2 \pi n_{1}}{\lambda_{0}} x \sin \theta_{1}\right)} e^{-\frac{2 \pi}{\lambda_{0}} z\left(n_{1}^{2} \sin ^{2} \theta_{1}-n_{2}^{2}\right)^{\frac{1}{2}}} \quad \text { (Sub. out } k_{2}\right) \\
\end{array}
$$

By defining $\delta$ to be the penetration depth of the evanescent field into the secondary (rarer) medium, and representing it as shown in Equation (3.37), the final expression of the Electric field in the secondary medium can be represented as shown in Equation (3.38). Additionally, $\delta$ is the depth at which the amplitude of the field reduces to $1 / e$ of its maximum value

$$
\begin{gathered}
\delta=\frac{\lambda_{0}}{2 \pi\left(n_{1}^{2} \sin ^{2} \theta_{1}-n_{2}^{2}\right)^{\frac{1}{2}}} \\
\therefore \boldsymbol{E}_{0,2}=\mathcal{E}_{0,2} e^{\mathrm{i}\left(\omega t-\frac{2 \pi n_{1}}{\lambda_{0}} \sin \theta_{1} x\right)} e^{-\frac{z}{\delta}}
\end{gathered}
$$

Many sources find it convenient to represent the Evanescent field in terms of irradiance, rather than Electric field intensity [91, 144]. The relationship between 
Irradiance and the Electric field is derived from the Poynting vector, as shown below.

$$
\begin{array}{rlr}
I & =\frac{1}{2} n \mathcal{Y} \boldsymbol{E} \boldsymbol{E}^{*} & \text { (From Eq. A.31) } \\
& =\frac{1}{2} n \sqrt{\frac{\varepsilon_{0}}{\mu_{0}}}|\boldsymbol{E}|^{2} & (\text { Sub. for } \mathcal{Y}) \\
& =\frac{1}{2} n \sqrt{\varepsilon_{0}^{2} c^{2}}|\boldsymbol{E}|^{2} & \left(\mu_{0}=\frac{1}{\varepsilon_{0} c^{2}}\right) \\
\therefore I & =\frac{1}{2} n \varepsilon_{0} c|\boldsymbol{E}|^{2}
\end{array}
$$

From here, it is possible to substitute in $\boldsymbol{E}_{0,2}$ and simplify the expression by defining $I_{0}$ to be the incident irradiance, represented as shown in Equation (3.41). Doing so yields Equation (3.40), which is in the same form as a simplified version of Beer's law.

$$
\begin{aligned}
I & =\frac{1}{2} n \varepsilon_{0} c\left[\boldsymbol{E}_{0,2}\right]^{2} \\
& =\frac{1}{2} n \varepsilon_{0} c\left[\mathcal{E}_{0,2}^{2} e^{2 \mathrm{i}\left(\omega t-\frac{2 \pi n_{1}}{\lambda_{0}} \sin \theta_{1} x\right)} e^{-\frac{2 z}{\delta}}\right] \\
\therefore I & =I_{0} e^{-\frac{2 z}{\delta}}
\end{aligned}
$$

$$
\text { where: } I_{0}=\frac{1}{2} n \varepsilon_{0} c\left[\mathcal{E}_{0,2}^{2} e^{2 \mathrm{i}\left(\omega t-\frac{2 \pi n_{1}}{\lambda_{0}} \sin \theta_{1} x\right)}\right]
$$

It is important to note that both $\delta$ and $I$ are strongly dependent upon the incident angle. Figure 3.6 demonstrates the penetration depth for a beam of $532 \mathrm{~nm}$ light incident upon a BK7 glass $(n=1.519)$ and air $(n=1.0)$ interface. The penetration depth decays quickly from a mathematically infinite value at the critical angle $\theta_{c}=41.17^{\circ}$. Near the critical angle, the penetration depth is on the order of the wavelength of the incident beam; however, at greater incident angles, the pen- 


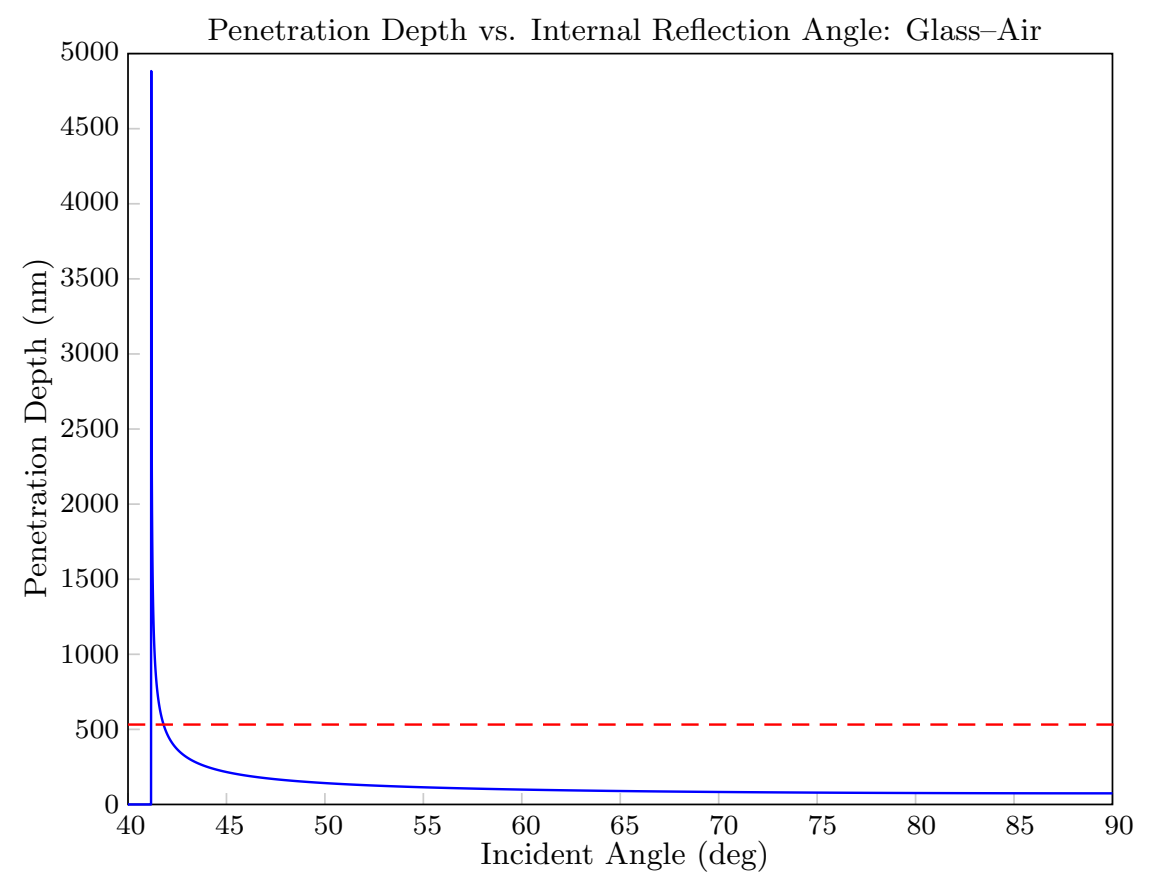

FIGURE 3.6 The penetration depth of the evanescent field created by totally internally reflecting $532 \mathrm{~nm}$ light is on the order of the wavelength of the beam near the critical angle, but decays quickly to an asymptote around $74.05 \mathrm{~nm}$.

etration depth reaches a horizontal asymptote around $74.05 \mathrm{~nm}$. Figure 3.7 depicts the relative penetration depths for each of three interfaces: glass-air, glass-polymer $(n=1.32)$, and glass-silver. Although TIR occurs throughout a larger portion of the angular spectrum for the glass-silver interface than for the other two interfaces, the evanescent field penetration depth decays to a shorter horizontal asymptote. Consequently, a relatively thin layer of silver around $60 \mathrm{~nm}$ might make for a sufficiently thick cladding layer as the $1 / e$ depth would be shallower than the thickness for most of the angular spectrum.

$$
\boldsymbol{E}_{0,2}=\mathcal{E}_{0,2} e^{\mathrm{i}\left(\omega t-\frac{2 \pi n_{1}}{\lambda_{0}} x \sin \theta_{1}\right)} e^{-\frac{2 \pi}{\lambda_{0}} z\left(n_{1}^{2} \sin ^{2} \theta_{1}-n_{2}^{2}+\kappa_{2}^{2}-2 \mathrm{in} n_{2} \kappa_{2}\right)^{\frac{1}{2}}}
$$




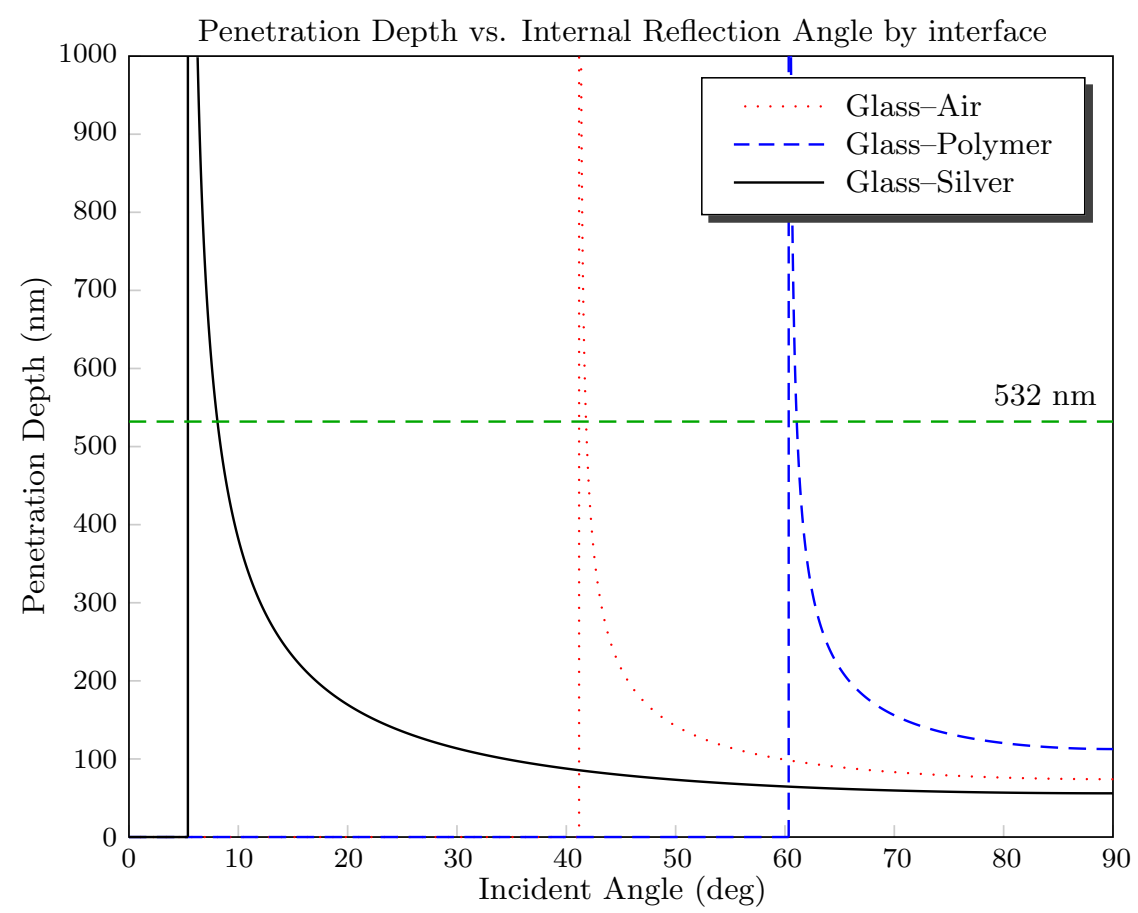

FIGURE 3.7 The penetration depth of the evanescent field created by totally internally reflecting $532 \mathrm{~nm}$ light decays slower for the Glass-Silver interface, but reaches a shallower horizontal asymptote than the Glass-Air and Glass-Polymer interfaces.

It is also worth considering that $\delta$, in this case, is calculated assuming that the secondary medium is non-absorbing. However, for metal clad waveguides, that may not necessary be applicable. Equation (3.42) represents an alternative expression for secondary materials that have a degree of optical absorption, denoted by the $\kappa$ term from the complex expression of the refractive index $(\widetilde{n}=n+\mathrm{i} \kappa)$ [70]. The optical absorption decreases the penetration depth of the evanescent field and forces the field to propagate at a slight angle to the interface, rather than along it as in the case of a non-absorbing rarer medium, which is not reflected in the graph shown in Figure 3.7.

For the mathematically inclined, this description of the evanescent field may be sufficient; however, an alternative conceptual approach would be to simply consider the photon to be a packet of energy propagating toward the interface of the two 
media. As the photon undergoes TIR, the momentum of the packet causes a portion of the energy to penetrate into the second medium, whereas the majority of the packet remains within the initial material, as shown in Figure 3.8. The penetrating portion of the packet reaches into the medium in the form of an exponentially decaying field, which is only present while the photon is undergoing TIR. The use of the term "evanescent", which means "fleeting" or "temporary", is specifically because the nonpropagating field is only present during a TIR event.

Then, provided the external medium is of lower refractive index than the originating medium, the bulk of the packet reflects back away from the interface. However, the evanescent portion during its brief presence in the rarer medium, has the chance to interact with the external medium, typically in the form of optical absorption. This interaction is referred to as "evanescent leaking" and is usually a consideration to be minimized in waveguide design due to the potentially significant loss of energy, except in cases where transmission efficiency is not a limiting design factor. It is also worth noting that in a two-medium system, such as those considered above, the evanescent field does not transfer energy into the external medium, except in the case of optical absorption.

\subsubsection{Frustrated total internal reflection}

Whereas most waveguides are designed to limit evanescent effects due to the potential loss of energy into the surrounding materials, our waveguides utilize that loss as a means by which to deliver light into tissue through a process referred to as frustrated total internal reflection (FTIR) [145]. In this process, the thickness of the secondary 


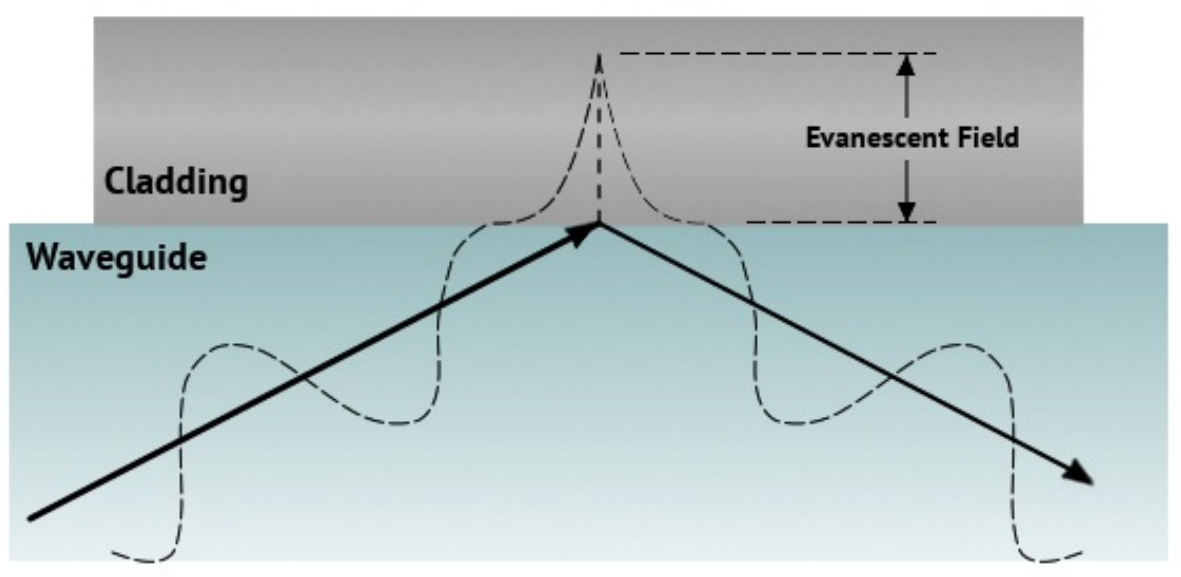

FIGURE 3.8 When a photon incident upon a boundary between two materials of differing refractive index reflects away from the boundary, a portion of the energy extends as an exponentially decaying field into the external medium.

medium is less than the penetration depth of the evanescent field. Consequently, the field extends into a third medium, where a percentage of the light is able to escape TIR and revert into a propagating photon within the third medium, as shown in Figure 3.9. This technique has been utilized in a variety of applications including prism couplers, optical filters and switches, biosensors, and in optically characterizing thin films [144, 146-151]. Moreover, it is also the basis upon which Photon Tunneling Microscopy functions [152].

Figure 3.9 shows that as the wave of light approaches the waveguide-film interface, a portion of the wave extends past the interface, forming the evanescent field as a decaying exponential field. When the film thickness, $d$, is sufficiently small, the peak of the evanescent wave extends beyond the thin film and into the tissue. Then, provided $n_{3}>n_{1}$, the evanescent peak reverts back into a propagating wave within the tissue, having "optically tunneled" through the thin film since the light was never 


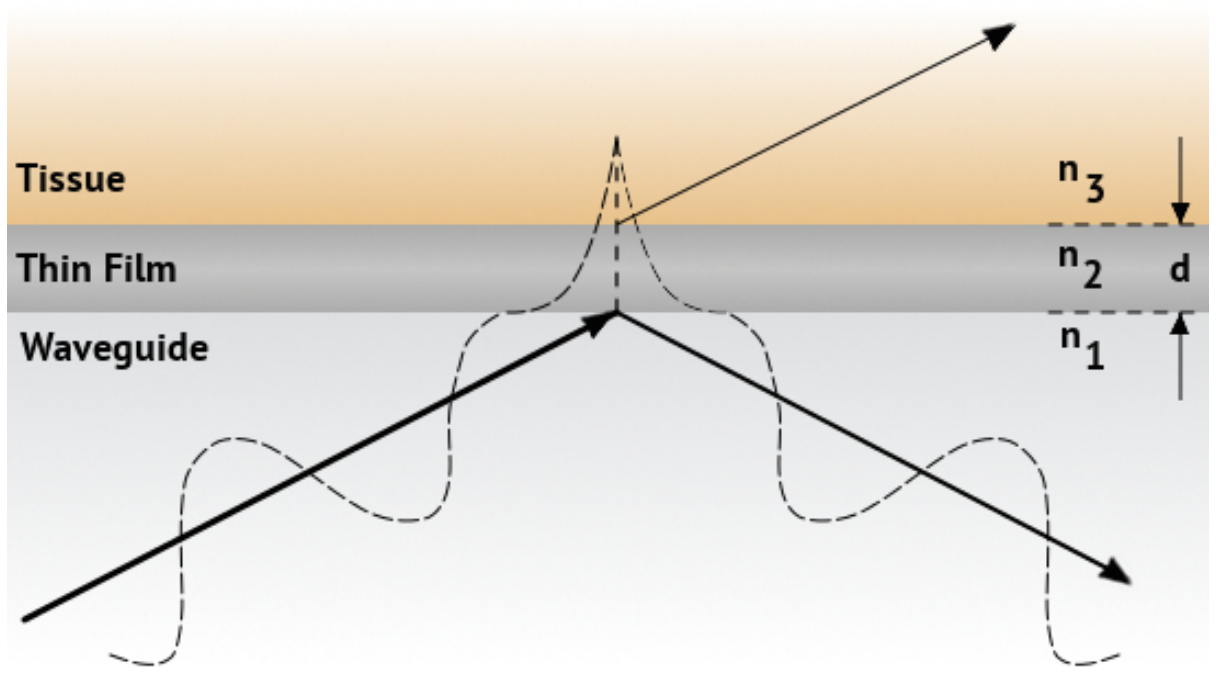

FigURE 3.9 If the boundary material is sufficiently thin, a portion of the evanescent field may penetrate into the external medium and revert into a propagating photon.

propagating within the film; although it is also called quantum tunneling, since the effect is analogous to that observed by electrons under similar situations [95].

In order to mathematically demonstrate this effect, we must derive an expression for the Transmission into the third external medium. Consequently, it is necessary to consider the reflectivity at the interfaces between each subsequent layer. It is convenient, therefore, to start by considering Försterling's solution for the amplitude reflection coefficient of a thin film between two semi-infinite isotropic dielectric media, shown in Equation (3.43) [153]. Figure 3.10 depicts the tri-layer boundary conditions considered in the derivation of the optical tunneling effect.

$$
\begin{array}{r}
r e^{\mathrm{i} \delta}=\frac{r_{12}+r_{23} e^{-\mathrm{i} x}}{1+r_{12} r_{23} e^{-\mathrm{i} x}} \\
\text { wherein: } x=\frac{4 \pi n_{2} d}{\lambda_{0}} \cos \theta_{2}
\end{array}
$$




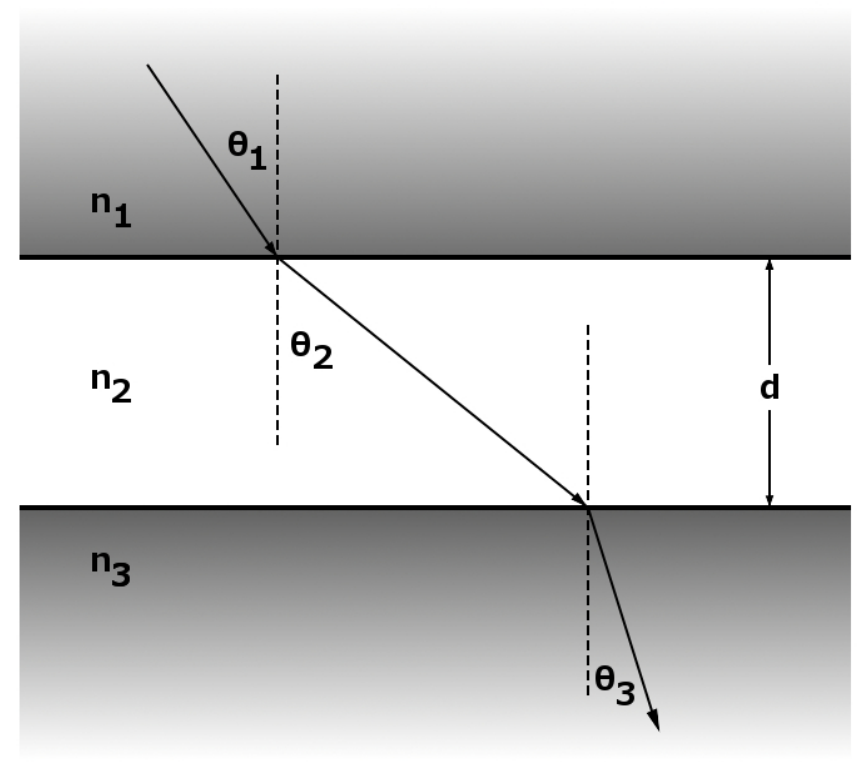

FIgURE 3.10 The diagram depicting the boundary conditions for FTIR is similar to a simple case of double refraction through an intermediary medium; however, under TIR, the beam technically does not propagate within the secondary medium $n_{2}$.

In this expression, $r_{12}$ and $r_{23}$ represent the complex reflection coefficients at each boundary $(1,2)$ and $(2,3)$ respectively, whereas $\delta$ represents the change in phase upon reflection, and $d$ represents the intermediate film thickness. It is important to note that the evanescent field within the second medium is not a propagating beam, and therefore $\theta_{2}$ has no real value. Consequently, using Snell's Law (Eq. 3.34) and substituting $x^{\prime}=\mathrm{i} x$, it is useful to rewrite Equation (3.44) in the form shown in Equation (3.45).

$$
x^{\prime}=+\frac{4 \pi d}{\lambda_{0}} \sqrt{n_{1}^{2} \sin ^{2} \theta_{1}-n_{2}^{2}}
$$

In this form, it is necessary to specify that $x^{\prime}$ adopt only the positive root, as a negative root results in an evanescent amplitude that increases to infinity. Addition- 
ally, in order to obtain an expression for the transmission through the film, we must first rewrite Eq. (3.43) by defining $r_{12}=e^{\mathrm{i} \delta_{12}}$ and $r_{23}=-r_{32}=e^{\mathrm{i} \delta_{32}}$, based on the fact that only the phase of the wave changes upon TIR along with Stoke's principle of reversibility.

$$
r e^{\mathrm{i} \delta}=\frac{e^{\mathrm{i} \delta_{12}}-e^{\mathrm{i} \delta_{32}} e^{-x^{\prime}}}{1-e^{\mathrm{i}\left(\delta_{12}+\delta_{32}\right)} e^{-x^{\prime}}}
$$

Consequently, following the derivation presented by Court et al., utilizing the Fresnel equation for s-polarized light (Eq. 3.26, 3.27), it is possible to determine an expression for the transmission through the intermediary film into the external medium for TE polarized light [145]. The resulting expression is given by Equation (3.47). A similar equation may also be derived for p-polarized light. Additionally, it is important to note that this equation assumes that the three media are optically transparent and have negligible absorption; however, a similar representation may be derived by using the complex form of the refractive index, $(\widetilde{n}=n+\mathrm{i} \kappa)$, instead. 


$$
T_{s}=\frac{1}{\alpha \sinh y+\beta}
$$

$$
\text { wherein: } \begin{aligned}
y & =\frac{2 \pi n_{2} d}{\lambda_{0}} \sqrt{N^{2} \sin ^{2} \theta_{1}-1} \\
\alpha_{s} & =\frac{\left(N^{2}-1\right)\left(\eta^{2} N^{2}-1\right)}{4 N^{2} \cos \theta_{1}\left(N^{2} \sin ^{2} \theta_{1}-1\right) \sqrt{\eta^{2}-\sin ^{2} \theta_{1}}} \\
\beta_{s} & =\frac{\left(\sqrt{\eta^{2}-\sin ^{2} \theta_{1}}+\cos \theta_{1}\right)^{2}}{4 \cos \theta_{1} \sqrt{\eta^{2}-\sin ^{2} \theta_{1}}} \\
N & =\frac{n_{1}}{n_{2}} \\
\eta & =\frac{n_{3}}{n_{1}}
\end{aligned}
$$

It is important to note that the intensity of the new ray of light in the third medium is dependent upon only the portion of the evanescent wave that penetrates into the third medium. Consequently, the majority of the light will be reflected back within the waveguide. Also, for our applications, biological tissue will likely have a refractive index lower than that of the waveguide, which would otherwise not allow for the tunneling to occur. However, in order to account for that issue, real tissue trials would involve an oil or optical gel specifically chosen to have a higher relative refractive index.

\subsubsection{Contact-based transmission}

Selective-release waveguides represent an amalgam of the fundamental optical concepts discussed above, in which laser light reflects between opposing surfaces of a 
planar substrate until undergoing FTIR and tunneling out through a designated active area into an external medium. Such waveguides are designed to have a relatively thick cladding covering the majority of their surface area to guarantee TIR along their length; however, within the active area located on one of their larger facets, the cladding is tailored to be considerably thinner, permitting FTIR to occur and thereby facilitating indirect light transmission into the targeted irradiation zone (TIZ). Transmitting light in such a manner effectively encloses the optical system, as compared to traditional free-space propagation modalities, by reducing the operational distance to 0 . Doing so circumvents many of the aforementioned complications presented by contemporary approaches, as discussed in Section 1.3.2, the most prevalent of which involved the potential for hazardous reflections from the surface of the tissue, which is rendered exceedingly unlikely assuming proper implementation of the SRW. Figure 3.11 demonstrates an application of a planar SRW for irradiating a epidermal and dermal tissue. Utilizing the SRW in this manner prevents any back-reflecting photons from exiting the tissue through the rear face of the waveguide, and in doing so averts the possibility for accidental exposure, representing a dramatic improvement in operational safety over conventional systems.

Moreover, by minimizing the operational distance of the optical system, this technique also eliminates the tissue-air interface in favor of a tissue-waveguide interface. Although not specifically investigated in this dissertation, the exchange of these two interfaces represents the replacement of thermal convection between the surrounding air and the tissue with thermal conduction between a solid substrate and the tissue. In this manner, it may be possible to minimize the generation of 


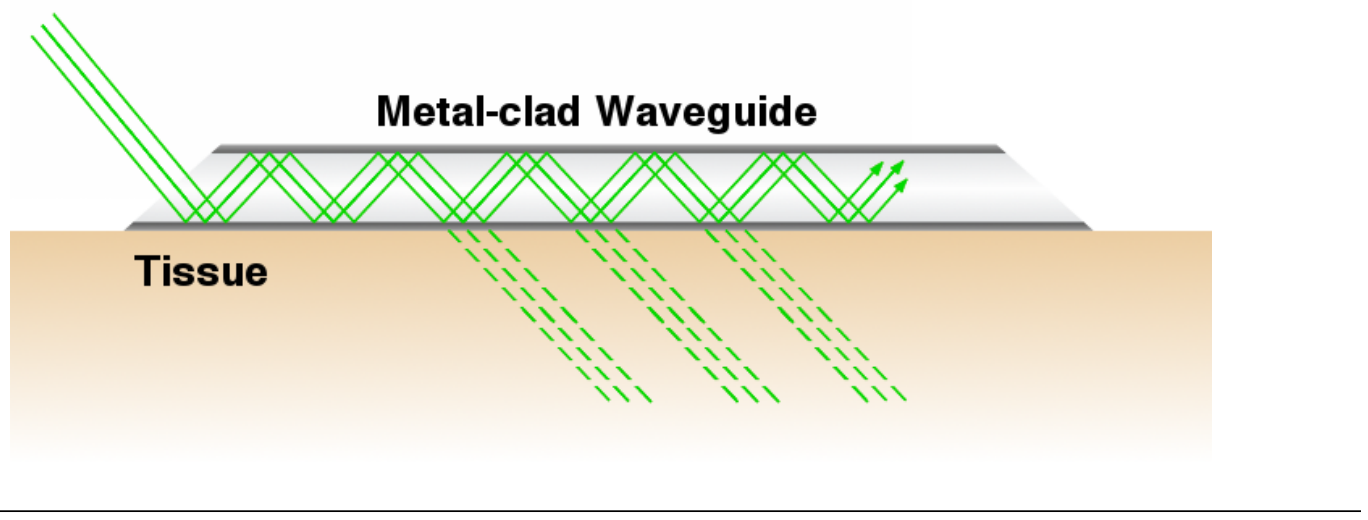

FIgURE 3.11 Transmitting light into tissue through physical contact of a planar SRW prevents any back-reflecting photons from escaping the confinement around the TIZ.

the surface plume that accompanies laser ablation, the frosting effect observed as a result of vaporization at the tissue surface, or the carbonization and inflammatory effects corresponding to photothermally generated heat within the tissue, each of which effects is strongly related to poor thermal conductivity at the tissue-air interface. Furthermore, as with the other secondary technologies incorporated behind SRW's that will be discussed in the chapters to follow, tissue irradiation through direct contact allows for the simultaneous application of contact-based cooling technologies that would otherwise occlude the optical path in contemporary light transmission modalities. Such techniques have already been clinically demonstrated to further reduce thermal tissue damage, but have seen limited implementation due to the operational constraints imposed by free-space propagation [19, 154].

\subsubsection{Objectives}

In applications such as that shown in Figure 3.11, SRW's capitalize on the evanescent leaking effect to selectively transmit light into the TIZ exclusively through 
a non-normative exit aperture, while conveniently mitigating detrimental side-effects of traditional methods. Consequently, for the purposes of waveguide characterization, it was hypothesized in this developmental study that the composition and thickness of the films within the defined active area would strongly correlate with the amount of light transmission into the external medium. Although prior experiments had demonstrated the potential to govern contact-based transmission by regulating active area geometry, a direct correlation between curve between active area film thickness and transmitted optical intensity had yet to be established [1]. As such, it was the intention of this study to characterize the behavior of silver-clad optical substrates, first using the SEM-EDS analysis described in the previous chapter to properly characterize film thickness and deposition rates, and subsequently to measure transmitted laser intensity through samples of ex vivo porcine skin.

For the purposes of this study, the selective-release waveguides were fabricated using fused silica (FS) substrates custom ordered from Opco Laboratories. The substrates incorporated $45^{\circ}$ entrance apertures, akin to that shown in Figure 3.11, and were then clad in thin films of titanium and silver. The silver was identified as a cladding layer due to its high reflectivity and comparatively low refractive index $\left(n_{A g}=0.143\right)$ at the experimental lasing wavelength of $532 \mathrm{~nm}$ as compared to that of FS $\left(n_{F S}=1.4607\right)$; whereas the titanium was simply utilized as a thin adhesion layer for the outermost silver films, which do not readily adhere to glass substrates $[72]$.

Figure 3.11 demonstrates that the beam was also forced to propagate along the length of the waveguides, escaping only through the designated active areas. For the 
purposes of this study, the active areas were formed by masking off a rectangular area of the substrate using Kapton tape during the cladding deposition process, preserving the bare glass surface below. Ten such waveguides were fabricated, after which nine of the waveguide active areas received an additional silver coating ranging in thickness from 5 to $60 \mathrm{~nm}$, while the remaining tenth waveguide was maintained with a bare glass active area to serve as a control for maximum refracted transmission. The waveguides were tested using porcine tissue samples to measure the magnitude of light transmission through each of the active area geometries and thereby establish a calibration curve for metal clad SRW operation.

\subsection{Materials and methods}

The sections that follow describe the experimental protocols involved in empirically establishing the relationship between SRW active area film geometry and light transmission through samples of ex vivo porcine skin. Additionally, the MATLAB code used to analyze the data and correct for systematic errors recorded by LabVIEW is included in Appendix B.

\subsubsection{Porcine tissue samples}

Large sections of ex vivo porcine skin were donated for this research project from the Mizzou Meat Market (College of Agriculture, Food, and Natural Resources at the University of Missouri). The skin was disinfected in a $1.75 \%$ ethanol solution for 2 minutes prior to processing for experimentation. Following disinfection, the sections were trimmed down to a thickness of $4 \mathrm{~mm}$, incorporating epidermal, dermal, and 


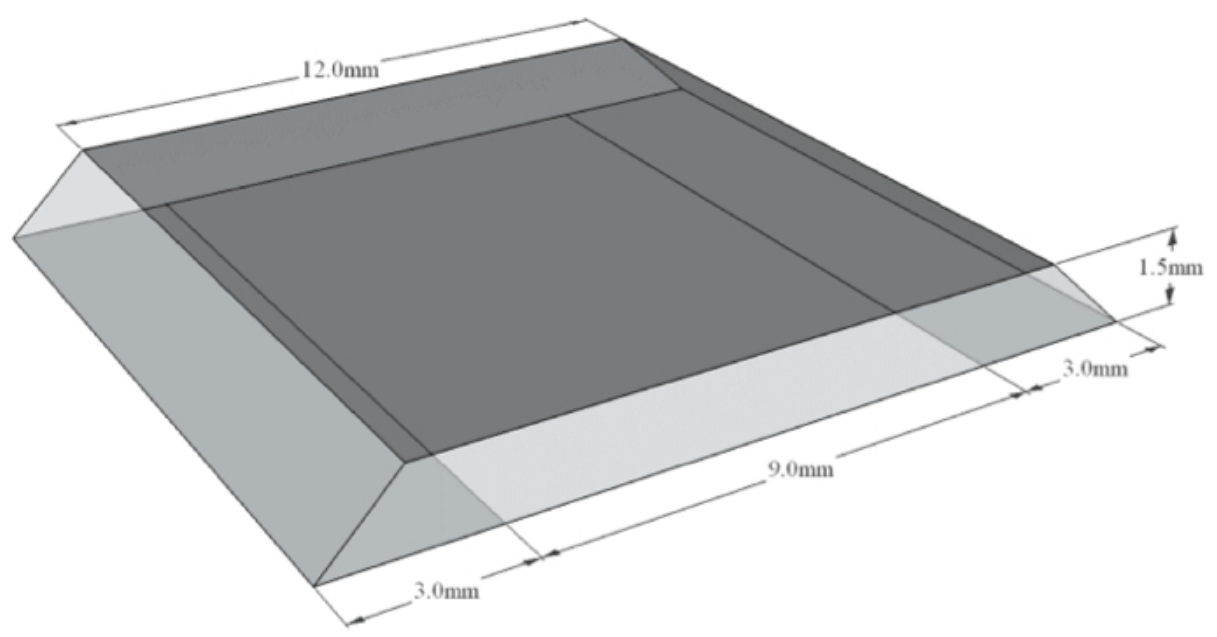

FIGURE 3.12 Planar substrates of fused silica incorporated $45^{\circ}$ angled faces at two opposing ends and were then clad in films of titanium and silver, while preserving rectangular sections on one face for use as active areas.

adipose tissue, and cut into $40 \mathrm{~mm}$ square samples. Originally, each sample was held taut and adhered at the edges with superglue over a thin fiberglass mesh to individual 3D-printed tissue mounts, which incorporated open apertures to allow for measurement of optical transmission through the tissue. The fiberglass mesh was included to provide additional support to the porcine skin and prevent potential indentation of the tissue during testing. Processed skin samples were wrapped in moist Kim wipes to prevent dehydration, enclosed in air-tight plastic bags, and stored in a freezer at $4^{\circ} \mathrm{C}$ until needed. Prior to use, samples were defrosted by running water over the plastic bag for $10-15$ min.

\subsubsection{Waveguide fabrication}

The optical waveguides consisted of fused silica substrates clad in films of silver and titanium. The fused silica substrates were custom ordered to be $15 \mathrm{~mm}$ optically 
transparent squares, $1.5 \mathrm{~mm}$ thick, with $45^{\circ}$ angled facets at two opposing ends to form a flat trapezoidal prism, as shown in Figure 3.12 (Opco Laboratory, Fitchburg, MA). The substrates were polished to within best efforts on flatness, 40/20 (scratch/dig) on all faces. The two angled entrance and exit apertures of the substrates were masked off with Kapton tape, along with a $9 \mathrm{~mm}$ x $15 \mathrm{~mm}$ rectangular section of the largest face, which would serve as the waveguide's active area. The substrates were then clad in thin films of titanium and silver using a cold sputtering vacuum deposition system (Quorum Technologies, K575X) to serve as mirrors on the bounding surfaces of the substrate. The titanium was deposited using a $99.998 \%$ pure Ti target at $90 \mathrm{~mA}$ for $60 \mathrm{~s}$, which formed a film $7 \mathrm{~nm}$ thick. The titanium acted as an adhesion layer for the silver, which was deposited from a 99.95\% pure Ag target at $30 \mathrm{~mA}$ for $240 \mathrm{~s}$ to form a much thicker layer approximately $240 \mathrm{~nm}$ thick. As such, laser light incident perpendicularly upon one of the angled faces would internally reflect along the waveguide to the exit aperture when exposed to air, but would transmit out through the exposed active area when in contact with tissue.

In order to govern the degree of optical transmission into the tissue, the composition of the active area cladding was regulated based on the presence and thickness of thin films of silver. Waveguides were fabricated with active area film thicknesses of 5 , $10,15,20,25,30,40,50$, and $60 \mathrm{~nm}$ while maintaining a bare active area waveguide to use as a control, which is denoted as either the "control waveguide" or a $0 \mathrm{~nm}$ active area waveguide. 


\subsubsection{Experimental apparatus}

The goal of these experiments was to investigate the efficacy of regulating light transmission into porcine skin samples by means of controlling the thickness of silver films in the active areas of ten waveguide substrates with the active area geometries defined above. Accordingly, pulsed laser light, generated by a Q-switched Nd:YAG laser (Opotek, Vibrant 355 II) operated at a wavelength of $532 \mathrm{~nm}$ with a $5 \mathrm{~ns}$ pulse width was transmitted through porcine skin samples using each of the sample waveguides individually and measured with a reverse-biased Si photodiode (Thorlabs, DET100A) attached to an integrating sphere, as shown in Figure 3.13. The optical apparatus first incorporated a zero-order half-waveplate (Thorlabs, WPH05M-532) and polarizing beamsplitter cube (Thorlabs, CM1-PBS251). Rotating the waveplate within it's housing permitted variable control of the ratio of laser intensity transmitted down the train toward the samples and that transmitted toward a power meter (Thorlabs, PM100USB) and photodiode trigger (Thorlabs, DET10A). The light directed toward the samples was first transmitted through an anamorphic prism pair (Thorlabs, PS883-A), which compressed the beam profile from a circular Gaussian into an elliptical profile with narrow axis thickness of approximately $1.5 \mathrm{~mm}$, roughly equivalent to that of the waveguide substrate. Prior to coupling into the waveguide substrates, the beam was transmitted through a long-focus cylindrical lens (Thorlabs, LJ1558L2-A),

which collimated the beam to correct for the significant dispersive effects of the prism pair.

An optomechanical clamp was designed and 3D printed to permit rapid, reliable 


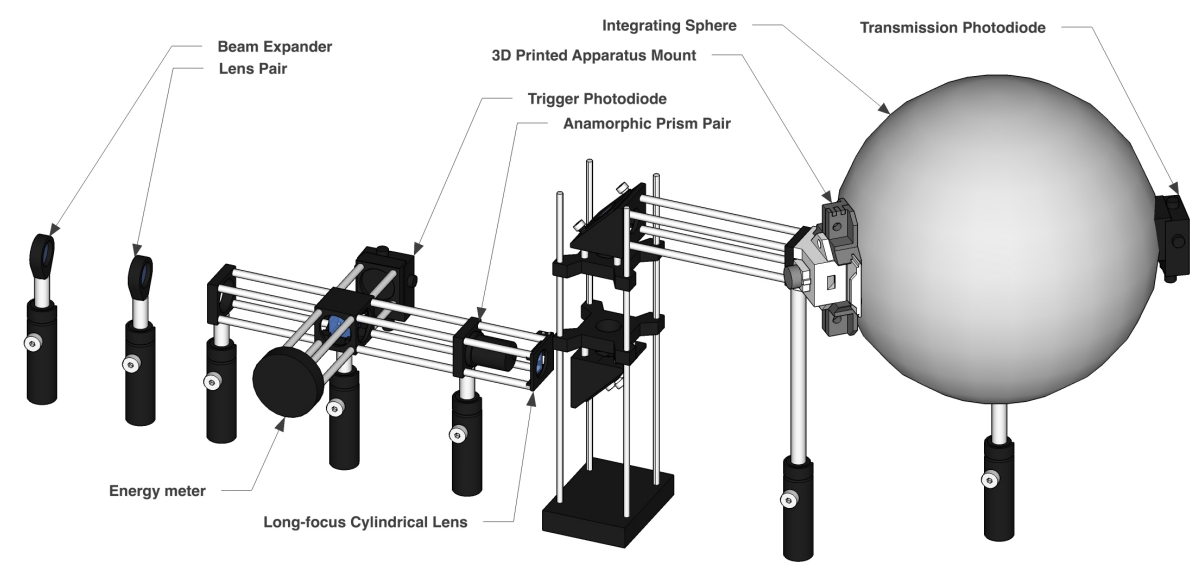

FiguRE 3.13 Pulsed light from a Q-Switched Nd:YAG laser was directed through a zeroorder half waveplate and polarizing beamsplitter cube, which transmitted the majority of the incident light toward an energy meter and a photodiode, whereas the remaining light transmitted into an anamorphic prism pair, cylindrical collimating lens, and was coupled into the waveguide.

interchanging of waveguide substrates without need for adhesive. The clamp bolted together with two screws positioned just above the substrates to apply pressure to the two unused side facets of each substrate. The clamp was designed to slot into a 3D printed optomechanical mount, which rigidly connected the optical train to the integrating sphere, while permitting the easy insertion of tissue sample cartridges. In this manner, the mount and clamp pair insert the waveguides into the optical train at a $45^{\circ}$ incline with respect to the incident beam, which ensured that the internal reflection angle of the beam within the substrate would also be $45^{\circ}$. To ensure sufficient optical coupling between the tissue samples and the optical waveguides, a thin layer of Cargille Type NVH immersion oil was applied between each interface. Cargille Type NVH is non-toxic, but is a significantly more viscous oil than Cargille Types A and B. This oil may not be absolutely necessary, but was utilized for consistency in comparing optical waveguides. 


\subsubsection{Experimental methodology}

The experimentation was performed by an automated LabVIEW program, which was designed to control laser operations and data collection, leaving only sample preparation and signal analysis to the scientist. The software controlled the Q-Switch delay of the laser, flashlamp activation, and laser firing by means of an RS-232 connection and serial input/output commands. Data was recorded using a 4 channel, $60 \mathrm{MHz}$ bandwidth USB data acquisition device (DAQ; Picoscope, 5442B) operating with 14 bit resolution. Data acquisition was triggered by the photodiode connected directly to the optical train, whereas incident laser energy was recorded for each shot using the USB connected power meter. The LabVIEW software was designed to fire a single shot and then record the peak photocurrent on channel A, the peak photovoltage on channel B, and the incident laser energy from the power meter before repeating for a total of 200 data points. The laser was fired at approximately $1 \mathrm{~Hz}$ to avoid any potential contamination of data from oversaturated detectors. The photocurrent was calculated by measuring the voltage drop across a $50 \Omega$ resistor in series with the photovoltage generated by the photodiode attached to the integrating sphere using a $1 \mathrm{X}$ voltage probe.

\subsection{Results}

The following sections describe the results of optical waveguide transmission studies performed using ex vivo samples of porcine skin, consisting of epidermal, dermal, and adipose tissues. The project progressed in two distinct phases of development: (1) empirically establishing the technique as appropriate for light transmission 


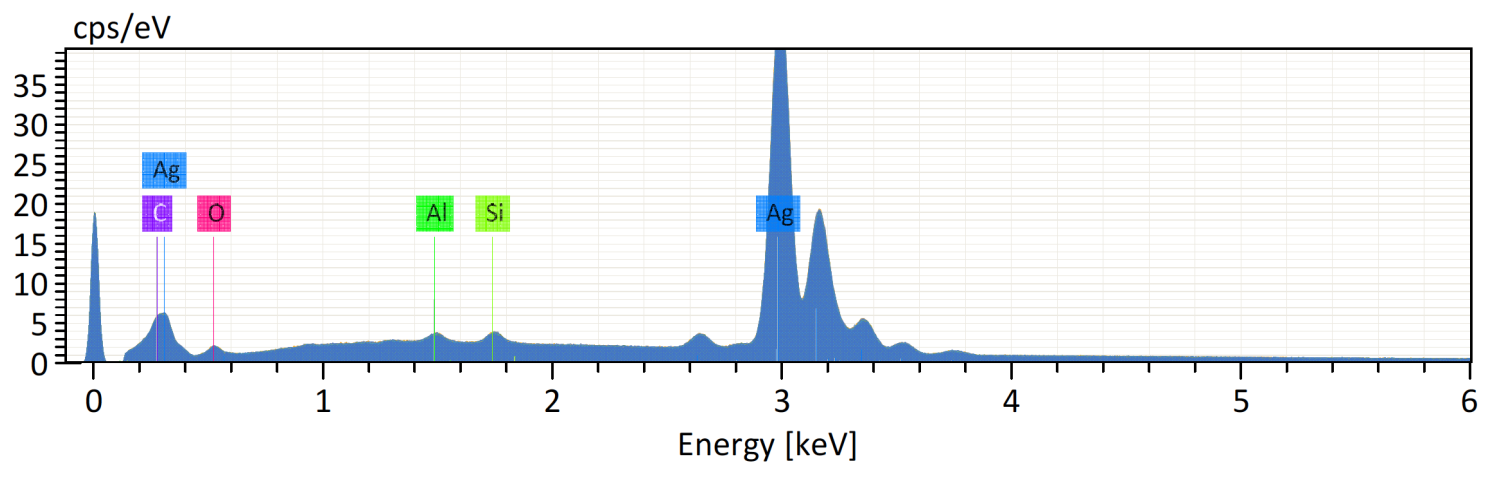

FIgURE 3.14 The SEM-EDS spectrum for silver deposition on silicon substrates at $30 \mathrm{~mA}$ for $240 \mathrm{~s}$ resulted in a film over 10 times thicker than that of titanium deposited for the same duration at higher current, indicating a significantly higher deposition rate for silver.

into tissue, as previous studies had only investigated tissue phantoms, and (2) fabricating a series of waveguides with active area thicknesses increasing at relatively small increments on the order of a few nanometers to concretely establish the relationship between film thickness and transmitted intensity.

During the first phase of the project, prior to waveguide fabrication, experiments were performed to empirically establish the deposition rates of the RF sputter coater used to clad the fused silica substrates in silver and titanium. These experiments were performed to obtain an accurate estimation of the film thickness in each active area without necessitating a direct measurement of every waveguide. SEM-EDS analysis, a metal film characterization technique discussed in Chapter 2, was performed to produce elemental spectra for substrates deposited with silver at $30 \mathrm{~mA}$ for 60,120 , 180 , and $240 \mathrm{~s}$ along with those deposited with titanium at $90 \mathrm{~mA}$ for the same durations. Figure 3.14 depicts a typical spectrum produced by the SEM-EDS software for the atomic ratios of a silver film on a silicon substrate deposited for $240 \mathrm{~s}$. The graph depicts an elemental ratio for $\mathrm{Si}: \mathrm{Ag}$ of $0.05: 1$, whereas when deposited for $60 \mathrm{~s}$ 


\begin{tabular}{lllll}
\hline \hline Duration & Atomic ratio [Si:Ti] & Ti Thickness & Atomic Ratio [Si:Ag] & Ag Thickness \\
\hline $60 \mathrm{~s}$ & $60.01: 1$ & $7 \mathrm{~nm}$ & $1.22: 1$ & $68 \mathrm{~nm}$ \\
$120 \mathrm{~s}$ & $39.02: 1$ & $13 \mathrm{~nm}$ & $0.35: 1$ & $115 \mathrm{~nm}$ \\
$180 \mathrm{~s}$ & $25.00: 1$ & $20 \mathrm{~nm}$ & $0.09: 1$ & $190 \mathrm{~nm}$ \\
$240 \mathrm{~s}$ & $19.51: 1$ & $25 \mathrm{~nm}$ & $0.05: 1$ & $280 \mathrm{~nm}$ \\
\hline \hline
\end{tabular}

TABLE 3.1 The tabulated atomic ratios determined by EDS data included alongside the corresponding film thicknesses calculated using the NIST DTSA-II spectral analysis software.

the ratio if $1.22: 1$, representing a decrease in interaction between the two elements with increasing film thickness. Table 3.1 includes the atomic ratios and calculated deposition rates for each silver and titanium of silicon substrates.

As discussed in Chapter 2, SEM-EDS is not a direct measure of film thickness, but instead relies on comparison of simulated models with experimental data. Accordingly, film thickness was determined by comparing the EDS spectra recorded with elemental simulations produced by the NIST DTSA-II software replicating the experimental conditions. These simulations were iteratively improved and narrowed until the simulated elemental ratios matched those recorded experimentally. The corresponding film thicknesses are included in Table 3.1, and were analyzed to determine deposition rates of $0.104 \mathrm{~nm} / \mathrm{s}$ for titanium on silicon at $90 \mathrm{~mA}$ and $1.17 \mathrm{~nm} / \mathrm{s}$ for silver on silicon at $30 \mathrm{~mA}$, assuming linear deposition rates due to RF sputtering occurring with constant current.

After establishing metal film deposition rates, waveguides were fabricated with active area film thicknesses of $0,10,20$, and $30 \mathrm{~nm}$, with $0 \mathrm{~nm}$ representing the bare substrate acting as the experimental control. Light was transmitted through the tissue samples and was recorded as a peak photovoltage intensity by the integrating sphere photodiode and the trigger photodiode. Owing to difficulties with the LabVIEW 


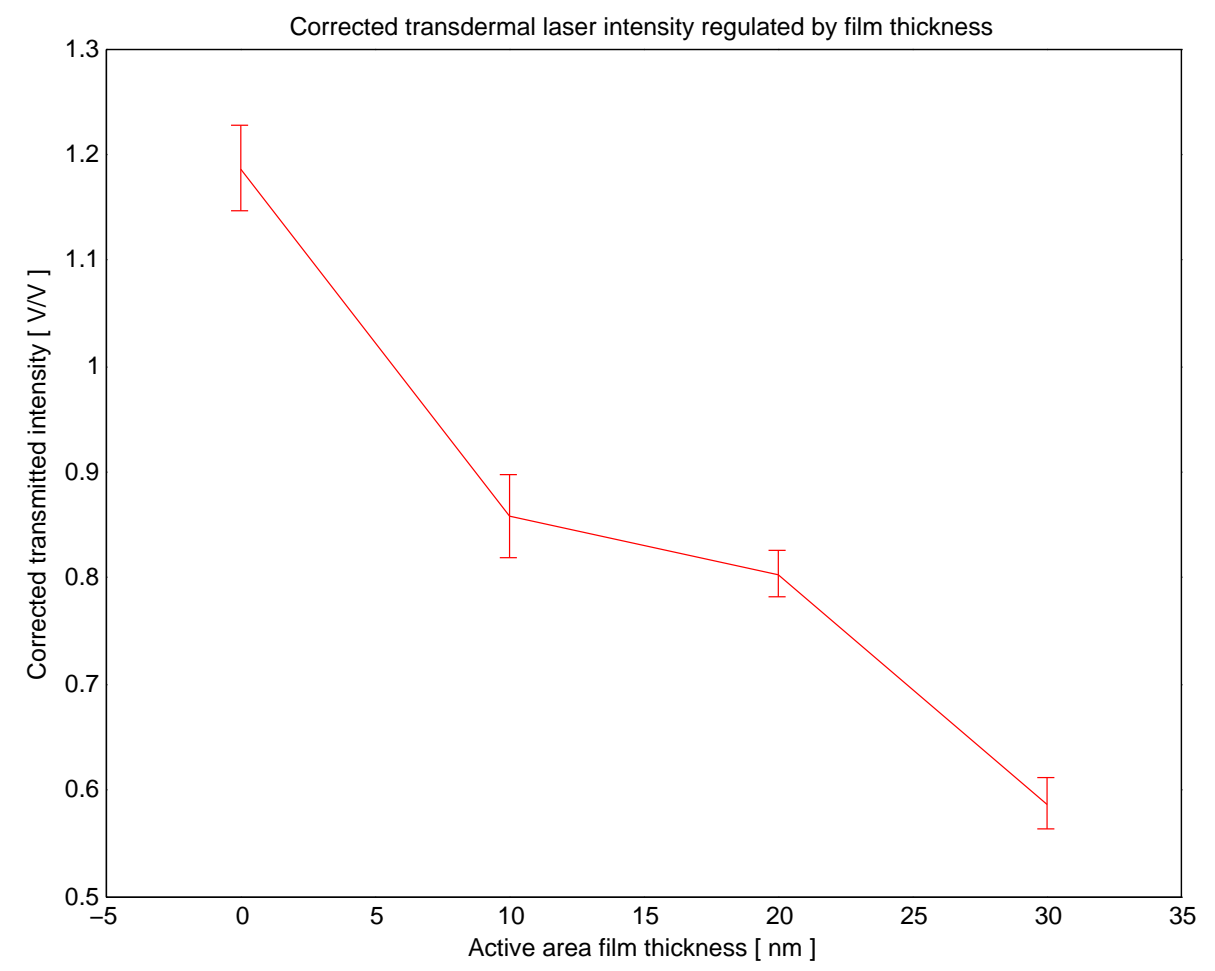

FiguRE 3.15 Preliminary experiments confirmed that the transmission of light through ex vivo samples of porcine skin decreased with increasing optical film thickness, as was predicted.

software provided by Thorlabs for the USB energy meter, pulse-to-pulse variations in laser intensity were corrected for by dividing the peak photovoltage of the sample photodiode by that of the trigger photodiode. Figure 3.15 depicts the results of this study, and demonstrates a distinct decrease in optical transmission with increasing film thickness, as was expected based on our prior work with optical tissue phantoms.

The data from phase 2 of this project is currently being collected and analyzed and will be incorporated into a later draft of this dissertation. A plot of the transmission of the ten waveguides is included in Figure 3.16; however, we are currently attempting to collect cleaner data with narrower error bars. It should be noted that this data was analyzed prior to our current laser energy measurement correction 


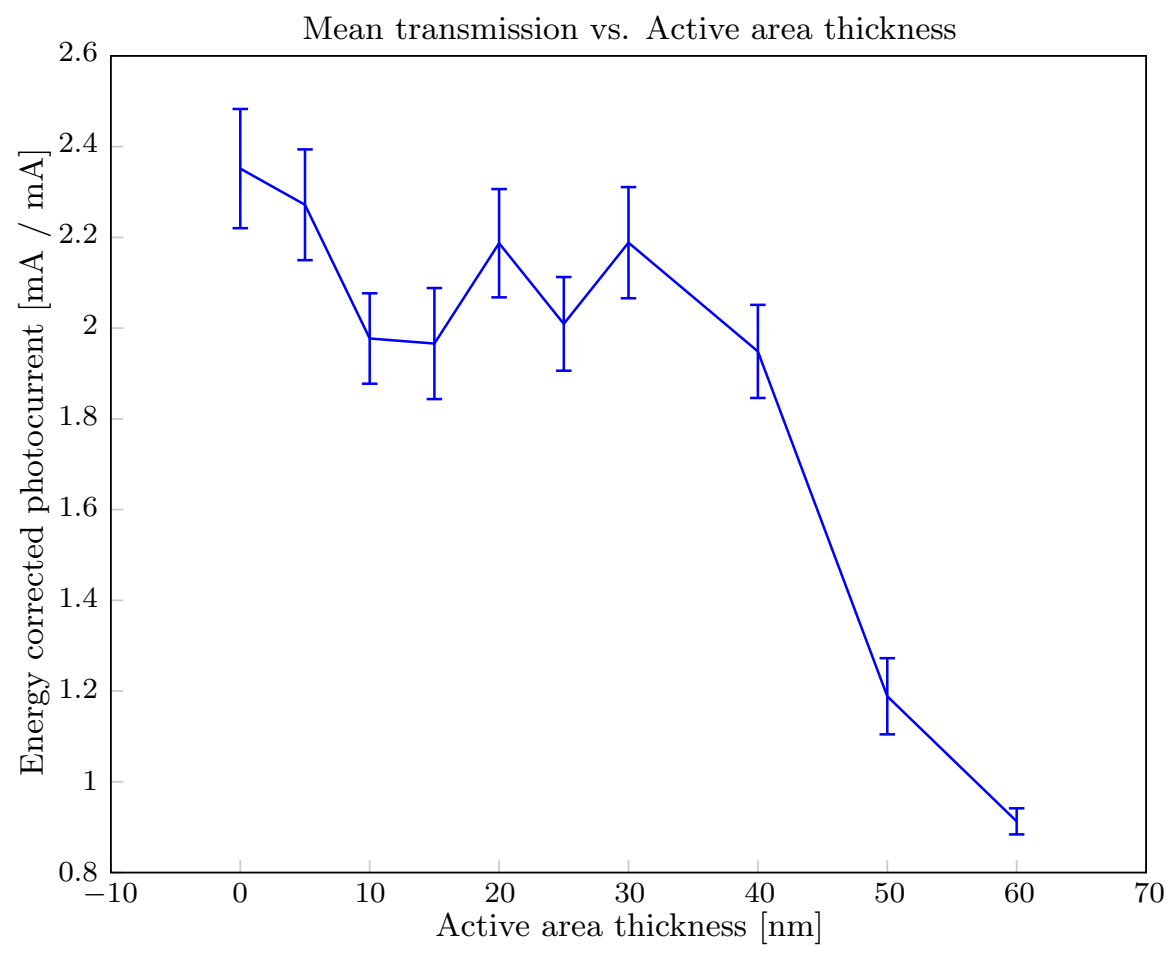

FiguRE 3.16 The transmission of light through thin film active areas decreases with increasing film thickness; however, the current data suggests that ultra thin films demonstrate a local minimum, which increases around $20 \mathrm{~nm}$ and then drops off rapidly thereafter.

paradigm, as discussed in Appendix C, so we will also be re-evaluating the data based on the new method of analysis.

\subsection{Discussion}

The principal ramification of the preliminary results included in Figure 3.15, is that they immediately demonstrated the potential for the waveguide technology to be utilized in tissue-based applications; whereas previous studies had been limited to artificial tissue simulations. The isolated and enclosed nature of the optical apparatus and photodiode within the integrating sphere concretely established that the waveguides each successfully transmitted light through the $4 \mathrm{~mm}$ thick samples of 
tissue Moreover, the decreasing transmission with respect to increasing film thickness suggests that the light underwent frustrated total internal reflection within the active area, which was regulated by controlling active area film geometry. An additional implication of the success of the preliminary data regarded the elimination of the operating distance between the laser source and the tissue. These results indicated contact-based transmission to be a viable technology, at least for the comparatively low intensities utilized in this study.

More recent optical transmission studies have been performed using waveguides with active area geometries from $0-60 \mathrm{~nm}$ in increments of $5 \mathrm{~nm}$ and have demonstrated some success; however, we have had problems with the reliability of the optical alignment and are currently revising our approach and collecting new data, which will be analyzed and incorporated into this dissertation. We have also recently serviced the laser by replacing the flashlamp and deionization cartridge, which will ideally improve the performance and reliability of the laser. 


\section{Chapter 4}

\section{Simultaneous paraxial ultrasonic pulsation to improve transdermal laser fluence}

Portions of this chapter have been reproduced with permission from the manuscript entitled Ultrasonic Modulation of Tissue Optical Properties in Ex Vivo Porcine Skin to Improve Transmitted Transdermal Laser Intensity, authored by Paul J.D. Whiteside, Chenxi Qian, Nicholas J. Golda, and Heather K. Hunt, published in the journal Lasers in Surgery and Medicine [155].

\subsection{Introduction}

Over the past few decades, the increasing demand for minimally invasive techniques for clinical or surgical applications has led to the development of a wide array of transdermal laser-based technologies. These technologies have been used for tissue resurfacing, tattoo and hair removal, treatment of pigmentary disorders, photothermal tumor ablation, and the treatment of vascular lesions $[2-4,156]$. The broad variety of optical therapeutic procedures is credited primarily to the laser devices' narrow wavelength bandwidth, which permits the specific targeting of individual chromophores based on their relative optical absorption spectra $[2,5]$. For exam- 
ple, the removal of green tattoo inks is typically best performed using either red or near-infrared laser light, owing to the tendency of green inks to absorb strongly in those wavelength regimes as compared to their absorption at shorter wavelengths in the ultraviolet to yellow regimes $[8,16]$.

However, for any given application, it is unlikely that the laser light will be perfectly, selectively absorbed by only the target of choice. Although the wavelength may be specifically chosen for greatest impact on the therapeutic target, there will inevitably be additional chromophores interspersed throughout the dermis and epidermis that may also absorb at the chosen wavelength. The three most predominant biological chromophores that detrimentally impact the efficacy of optically based procedures are hemoglobin, melanin, and water; a number of additional proteins and enzymes have been shown to absorb light within the UV spectrum as well [157-159]. Hemoglobin, melanin, and water vary in concentration based on both the patient's skin type and the relevant anatomical region; however, their absorptive properties, and therefore their impact on the procedure, are dependent upon only their molecular structure. Accordingly, each of these molecules exhibits a different absorption spectrum, as shown in Figure 4.1, with melanin absorbing strongly throughout the visible spectrum and hemoglobin demonstrating two slightly different spectra based on its state of oxygenation $[160,161]$. In the event that one or more of these spectra overlaps with that of the clinical target at the chosen wavelength, their presence in the tissue above the target will necessarily detract from the efficacy of the procedure not only by reducing the amount of light that reaches the target, but also by generating undesirable photothermal effects, such as carbonization and inflammation, which 


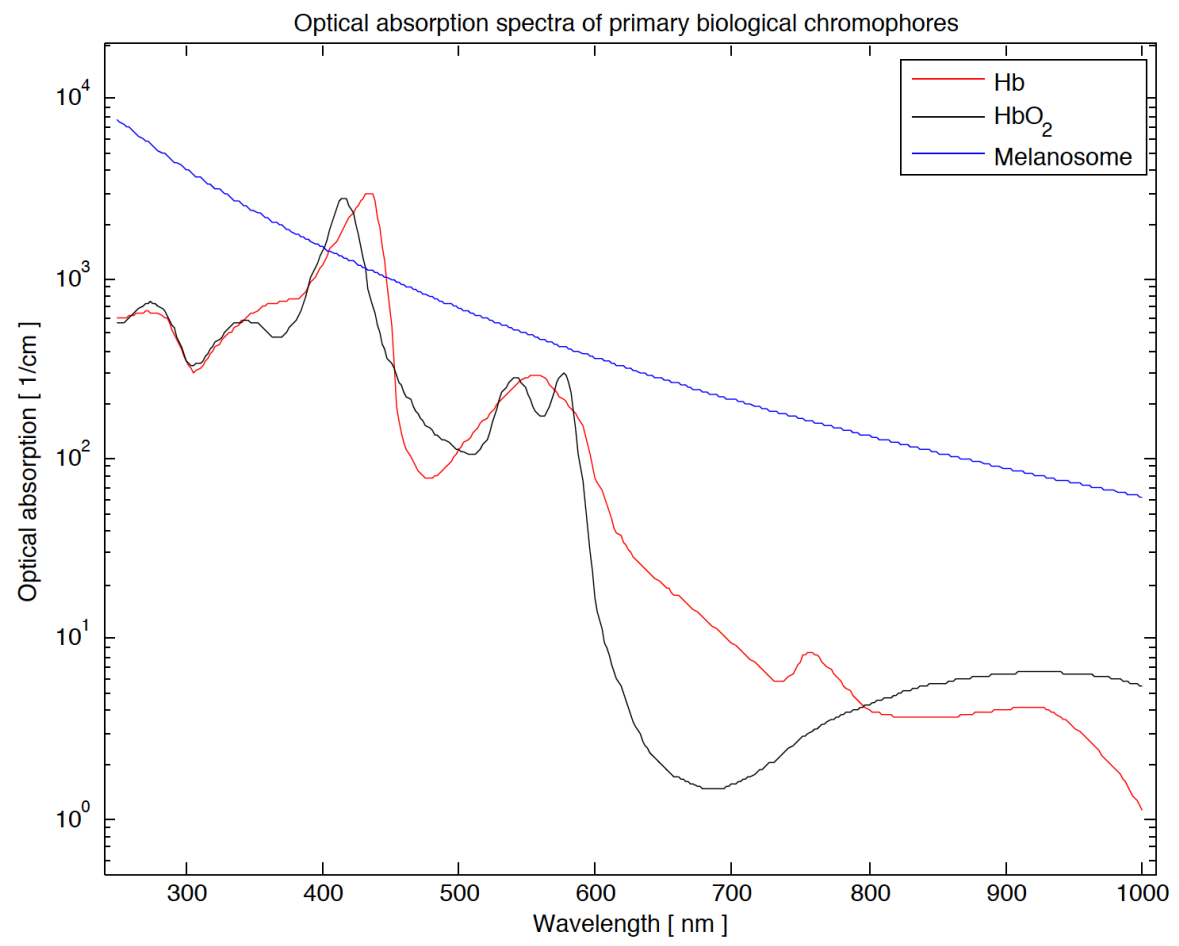

FIGURE 4.1 Whereas melanosomes in human skin are on average broadly absorbing throughout a wide range of wavelengths, hemoglobin exhibits peaks and valleys in both oxygenated and deoxygenated spectra, which may be exploited when selecting lasing wavelength.

thereby further exacerbate the attenuation of light $[9,35,43]$.

The clinical solution to this problem is typically to either increase the incident laser energy or otherwise reduce the laser spot size, both of which increase the laser energy density that reaches the clinical target [22]. Such practices serve as an expedient solution for an immediate clinical problem; however, they also intensify the detrimental side effects presented by unintended optical targets. This is particularly concerning in the case of epidermal melanin absorption. Melanin is a broadband optical absorber produced within the surface epidermal tissue, and is therefore likely to reside in the path between the laser source and the clinical optical target. Unsurprisingly, the efficacy of light-based techniques decreases dramatically for patients 
with higher concentrations of melanin (i.e. skin types higher on the Fitzpatrick scale) [162]. Moreover, the side effects resultant from melanin absorption also increase with local energy density, which can result in scarring or localized pigmentary changes (hypo- or hyperpigmentation) [56]. The combination of these two concerns renders most optical therapeutic techniques increasingly ineffective for a substantial portion of the global population.

Previous research has attempted to address this problem by optically clearing the tissue via chemical or mechanical means. For instance, a number of chemical optical clearing agents (OCA) have been investigated to increase optical transmittance in laboratory imaging techniques like Optical Coherence Tomography (OCT), including glycerol, glucose, polypropylene glycol (PPG), polyethylene glycol (PEG), dimethyl sulfoxide (DMSO), and oleic acid . However, for potential clinical applications, glycerol is typically preferred as it is biocompatible and considered a safe chemical agent by the Food and Drug Administration $[163,164,164,165]$. Yoon et al. demonstrated that the tissue optical clearing (TOC) effects of glycerol may be further improved by physically overcoming the barrier presented by the stratum corneum using a microneedle roller to introduce artificial microchannels in the tissue followed by the application of sonophoresis, which ultrasonically induces microcavitation within the tissue along with thermal and mechanical effects that help to promote permeability of OCAs into the tissue [166-169].

Purely mechanical methods have also been demonstrated; these aim to avoid any long-term chemical clearing by mechanically altering the tissue's local optical properties. Rylander et al. demonstrated two such mechanically-based tissue optical 
clearing devices (TOCD), one of which used an array of mechanical pin transducers to induce localized vacuum pressure, whereas the other used an array of ball lenses to focus light into the tissue while applying a vacuum pressure in the interstices between lenses [170]. Both of these TOCDs utilized the vacuum to stretch and compress the tissue while under radiant exposure, thereby displacing interstitial water and blood within the tissue and increasing light fluence through porcine skin samples [171]. Their work suggested that mechanical modification of optical properties had the potential to improve radiant throughput by reducing optical path length and potentially displacing chromophores.

Although both paradigms to tissue optical clearing have proven effective in prior investigations on in vitro, ex vivo, and in vivo tissues, each method presents potentially limiting factors or detrimental side effects. Of the two approaches, OCAs have been much more broadly investigated, which has yielded reports that their efficacy varies strongly with concentration. Even agents such as glycerol, which are considered relatively "safe," have been shown to significantly alter tissue structure or obstruct localized blood flow in high concentrations, while insufficiently clearing the tissue at lower concentrations; other OCAs may induce edema or suppuration, or result in tissue scarring [165]. Moreover, even when appropriate concentrations are utilized, the barrier presented by the stratum corneum often prevents or prolongs topical diffusion of OCAs into the tissue, which may necessitate invasive hypodermal injection of the OCA or lengthy pre-treatment with sonophoresis in order to effect optical clearing [172]. By contrast, mechanical TOCDs represent a relatively recent technology and accordingly have seen very limited development. Although publications have been 
generally positive, their efficacy in clinical therapeutics has yet to be demonstrated and improvements in transmitted intensity are postulated as related to observed decreases in optical scattering, tissue thickness, and changes in group refractive index $[171]$.

Therefore, the purpose of this research was to develop an alternative tissue optical clearing technique that would be predominantly mechanical in nature, but which could also be utilized alongside the application of an OCA if necessary. Whereas the aforementioned research by Yoon et al. involved the use of ultrasound to improve the diffusion of OCAs into the skin, the work presented herein instead focused only on the mechanical action of ultrasonic pulsation to locally alter the optical properties of the tissue through periodic compressions and rarefactions [173]. This technique builds upon precedent established by low intensity Ultrasound-Modulated Optical Tomography (UOT), wherein continuously pulsed ultrasound results in changes in mass density and the corresponding optical properties (i.e. refractive index, scattering coefficient, absorption coefficient), and changes in optical phase due to the displacement of scatterers and absorbers [174].

\subsubsection{Objectives}

Unlike sonophoresis-based methods, which are applied prior to laser treatment, this work investigated the potential for continuously pulsed ultrasound to mechanically modify the tissue throughout the course of the procedure. However, in established UOT publications, the ultrasound is typically applied to ex vivo tissue samples in water baths perpendicular to the direction of optical transmission, which 


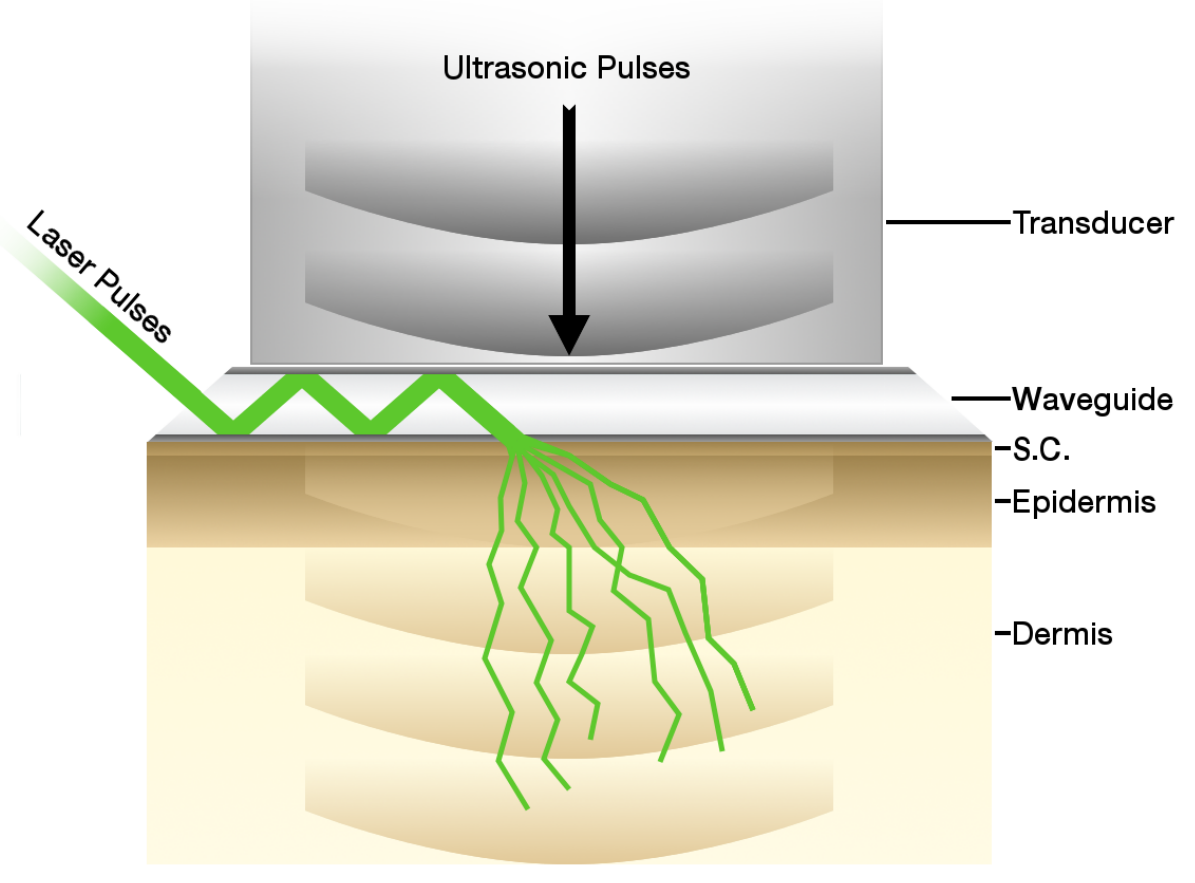

FIgURE 4.2 Using an optical waveguide permits the paraxial application of ultrasonic pulsation, which modulates the tissue's optical properties to improve transdermal laser transmission.

is patently not an option for any clinical application. To resolve this issue, planar optical waveguides were used to transmit laser light directly into the tissue through physical contact, which permitted the paraxial application of ultrasonic pulsation on the rear face of the waveguide, as shown in Figure 4.2 [175]. Although both these techniques had previously been independently established, this project hypothesized that their paraxial application might sufficiently alter the optical properties of the tissue such that total intensity of transmitted light through epidermal, dermal, and adipose tissue would be substantially increased.

As such, Q-switched Nd:YAG laser, similar to those used in clinical dermatology, was used to transmit pulses of light at a wavelength of $532 \mathrm{~nm}$ into samples of ex vivo porcine skin by means of a silvered acrylic optical waveguide, based on de- 
signs established in prior investigations $[1,66,83]$. The light was confined within the waveguide until reaching a pre-defined active area in contact with the tissue, whereafter the light refracted into the skin. Ultrasound was then applied on the rear face of the waveguide at a frequency of $510 \mathrm{kHz}$, varying the amplitude to determine what effect, if any, the ultrasound might have upon the transmission of light through the tissue.

\subsection{Phenomenological model}

Figure 4.2 depicts a theoretical means of implementing sonoillumination in which acoustic pressure is applied to the tissue by means of an ultrasonic transducer. The acoustic waves are coupled into the tissue through an optical selective release waveguide (SRW), which concept was discussed in Chapter 3. Moreover, the SRW simultaneously irradiates the tissue with light, which propagates through the ultrasonically modulated medium. The following phenomenological model represents the current understanding of the methods by which sonoillumination improves optical transmission through epidermal, dermal, and adipose tissues; although, the same principles can be translated to applications for other tissues and non-biological media.

\subsubsection{Established acousto-optic theory}

The ultrasonic modulation of transparent and turbid media for optical benefit has been extensively demonstrated in the realms of acousto-optics and ultrasoundmodulated optical tomography. Accordingly, the discussion of the mechanisms of sonoillumination are founded on established acousto-optic theory coupled with em- 
pirical observations recorded in lab.

Acousto-optics (A-O), not to be confused with opto-acoustics, principally regards the modulation of optical properties of transparent media by means of ultrasonic pulsation, and has been implemented to generate acoustically-induced diffraction gratings, deflectors, and optical modulators $[141,176,177]$. In such applications, acoustic pressure induces regional changes in optical refractive index within the regions of applied acoustic pressure, which will form a column when applied using an unfocused transducer or a hyperboloid when using a focused transducer, with the waist of the hyperboloid located at the focal point of the transducer. This change in refractive index is resultant from an acoustically induced strain within the medium, which alters the medium's local permittivity by means of varying the local material density [178]. Considering the strong relationship between material density and optical refractive index, which is a measure of the relative speed of light within a medium, any density alterations within a medium necessarily change the local relative speed of light due to an increase or decrease in the amount of material per unit of space that is present to hinder light transmission, leading to a direct relationship wherein $\Delta n / n=\Delta \rho / \rho[179]$.

When the acoustic field applied to a medium is nearly orthogonal to the incident light, the acoustic waveform can induce a gradient refractive index field (GRIF) consisting of intermittent regions of high pressure followed by comparatively low pressure, which forms a diffraction grating moving with the velocity of the acoustic wave in the medium [180]. For a plane wave propagating along the z-axis in a transparent medium, the refractive index becomes a function of both depth, $z$, and time, $t$, and 
can be expressed as Eq. 4.1,

$$
\begin{aligned}
n(z, t) & =n_{0}-\Delta n \cos \left(\Omega_{a} t-k_{a} z\right) \\
\text { wherein: } \Delta n & =\frac{1}{2} p_{z j} n_{0}^{3} S_{0}
\end{aligned}
$$

in which $\Omega_{a}$ is the angular frequency $(2 \pi f)$ and $k_{a}$ is the acoustic wavenumber $\left(2 \pi / \lambda_{a}\right)$, $\Delta n$ is the amplitude variation in the refractive index, $S_{0}$ is the amplitude of the acoustically-induced mechanical strain, and $p_{z j}$ is the photoelastic constant (i.e. the strain-optic coefficient) [178]. These equations are simplified and assume that the acoustic pressure is applied orthogonal to the surface of the medium by an unfocused acoustic source, and that the medium is homogenous. As such the equations omit deviations in the $\mathrm{x}$ - and $\mathrm{y}$ - directions; however, in practice, such deviations will occur due to inhomogeneities in the media involved. Additionally, the negative value indicates that a positive strain corresponds with a decrease in refractive index.

According to Eq. 4.1, the acoustic wavenumber, $k_{a}$, is a determining factor with regard to the depth-resolved change in refractive index. The ramification of this relationship is that the diffractive or deflective effects are directly affected by the properties of the acoustic waveform, including its amplitude, frequency, and wavelength, since $k_{a}=2 \pi f / c_{s}$, wherein $f$ represents the acoustic pulsation frequency and $c_{s}$ represents the speed of sound in the medium. This distinction has led to frequency regimes of differing effects, with Raman-Nath diffraction and Bragg diffraction. The 
two regimes are typically determined by the Klein-Cook parameter:

$$
\begin{aligned}
Q & =\frac{k_{a} l}{k_{l}} \\
& =\frac{\left(2 \pi f / c_{s}\right)^{2} l}{(2 \pi n / \lambda)} \\
\therefore Q & =\frac{2 \pi \lambda l f^{2}}{n c_{s}^{2}}
\end{aligned}
$$

wherein $k_{l}$ is the optical wavenumber $(2 \pi n / \lambda)$ and $l$ is the acousto-optic interaction length, which is defined to be the distance over which the ultrasound interacts with the light (e.g. the width of the acoustic column when the light is applied orthogonally). Utilizing this representation, Raman-Nath diffraction is observed for $\mathrm{Q} \ll 1$ and Bragg diffraction is observed for $\mathrm{Q} \gg 1$ [180]. In acoustically isotropic media, such as the crystals used in bench-top acousto-optics, these regimes typically correspond with frequencies less than $10 \mathrm{MHz}$ and greater than $100 \mathrm{MHz}$ respectively, with no clearly defined threshold between the two, but rather a gradual increase or decrease in the occurrence of the particular interference fringes produced by each type of diffraction, with $Q \approx 1$. However, Zakharov et al. have demonstrated that in elastically anisotropic media the intermediate regime occurs within a narrower frequency bandwidth and that the maximum intensity of diffracted light may be attained at lower frequencies, both of which may have significance for sonoillumination at higher frequencies $[180,181]$. 


\subsubsection{Established UOT theory}

Bench-top A-O typically involves the use of optically transparent media to induce an acoustically variable diffraction grating; however, ultrasound-modulated optical tomography (UOT) employs the techniques to improve imaging in ex vivo tomography of tissue structures $[179,182,183]$. Optical tomography traditionally suffers resolution losses due to scattering-induced destructive optical interference at the detector, which effect is referred to as "speckle" [184]. Although A-O focuses on modulating refractive index, in UOT ultrasonic pulsation is applied to ex vivo tissue samples to modulate the position of scattering elements within the tissue and thereby alter the speckle effects observed at the detector interface [185].

In addition to refractive index changes, the applied acoustic pressure wave also displaces optically scattering and absorbing matter within the medium [185]. Although the displacement is typically very small, on the order of tens or hundreds of nanometers, it is sufficient to periodically alter the conditions of optical collision events, which results in the blurring of the observed speckle. Moreover, the modulation of scatterer position can also result in a phase modulation of the optical field and a Doppler-shift in the optical frequency corresponding to the magnitude and frequency of the scattering wavevector [186]. This Doppler-shift can result in the generation of "acoustic sidebands" that are located as multiples of the acoustic propagation frequency around the primary incident laser frequency. In UOT, the Doppler-shift can be significant for reducing speckle in image formation, because frequency-shifted photons are less capable of destructively interfering with the primary optical frequency, 


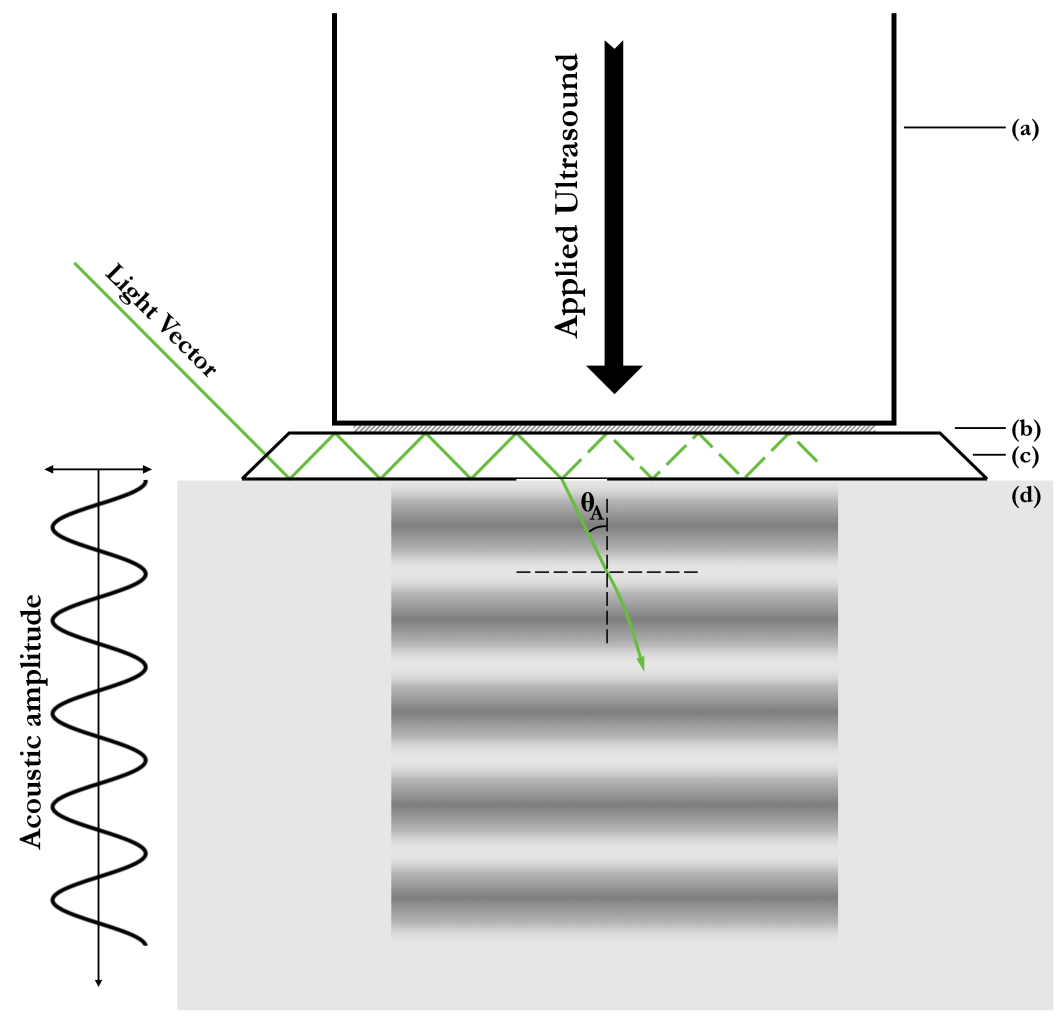

FIGURE 4.3 In sonoillumination, pulsed ultrasound is applied by an (a) an ultrasound source, via (b) an optional acoustic couplant, into (c) an optical selective-release waveguide, which transmits both light and acoustic pressure into (d) the target medium.

which can blur and effectively fill-in the imaging gaps left by destructive speckling.

\subsubsection{Mechanisms of sonoillumination}

The mechanisms of sonoillumination build on the principles established by acousto-optics and ultrasound-modulated optical tomography, while also representing a significant deviation from both. Figure 4.3 represents a theoretical diagram of sonoillumination theory, which incorporates both (1) regional changes in optical refractive index as predicted by acousto-optics, and (2) the displacement of optically scattering and absorbing matter as employed in UOT; however, sonoillumination also incorporates two additional effects regarding the anisotropy of the tissue and 
a frequency-dependent relationship with the transport mean free path of the light through the turbid medium, which will be discussed below.

\subsubsection{Refractive index modulation}

With regard to differentiating sonoillumination from the two established techniques, applications of A-O and UOT typically utilize cases wherein the angle between the incident light wavevector and the acoustic wavevector, represented in Figure 4.3 as $\theta_{A}$, is relatively close to orthogonal (i.e. close to $\pi / 2$ ) [141]. In A-O, this permits the light to transmit through the acoustically-induced pressure zones as if it were transmitting through a physical diffraction grating, with the distinct advantage of being able to change the properties of that grating in real time by altering the pulsation frequency, magnitude, or timing with respect to laser operation. Moreover, in UOT, the orthogonally applied acoustic pressure effectively shortens the magnitude of the scattering wavevector and thereby reduces the impact of collision events and destructive interference on the detected image. However, for the purposes of sonoillumination, the effective angle of incidence between the light transmitted into the medium and the acoustic propagation vector is of substantial significance. Specifically, in the theoretical diagram shown in Figure 4.3, the angle between the acoustic propagation vector and the incident light vector is significantly less than $\pi / 2$, which permits the acoustically varying GRIF generated within the tissue to behave less like a diffraction grating and more like a gradient refractive index lens (GRIN), continuously refracting the light as it propagates through the tissue.

Akin to the effect explained by Eq. 4.1 in transparent media, the GRIF is de- 
pendent upon the amplitude variation in the refractive index and the induced strain, both of which are directly dependent upon the applied acoustic waveform. For example, a continuous sinusoidal waveform, such as is depicted in Figure 4.3, induces a field that continuously changes from regions of greater density to those of lower density, with GRIF characteristics corresponding to those of the applied acoustic pressure with regard to frequency, wavelength, waveform, amplitude, and the direction of the acoustic propagation vector. Accordingly, the varying refractive index field may cause the incident light to undergo subsequent refraction throughout the tissue in a manner similar to light transmission through a physical GRIN lens, wherein the light propagation vector bends as it transmits through the continuous gradient [187]. Since the GRIF is not a physical constant, but is instead induced by means of a temporally and spatially variant acoustic waveform, differing applications may benefit from applying the GRIF such that it exhibits a desired refractive index or relative pressure at a specific location within the medium. For example, the GRIF may be applied such that there is a local refractive index maximum at the surface of the tissue, which would alter the optical boundary conditions between the tissue and external medium, thereby decreasing the optical reflection coefficient and the angle of refraction. Alternatively, the GRIF may be applied to exhibit a maximum at a defined distance below the surface in order to apply a mechanical strain to a specific optical target to improve a desired therapeutic effect, such as the photomechanical ablation of tattoo ink located within the dermis. 


\subsubsection{Alteration of optical collision events}

In addition to refractive index effects, the applied ultrasound also modulates the position of scatterers within the tissue, which can have a variety of ramifications that collectively contribute to improving optical transmission. As in UOT, the displacement of scatterers can result in a Doppler-shift in the frequency of the applied photons. Although the Doppler-shift is at least seven or eight orders of magnitude smaller than the primary optical frequency, which will have negligible impact with regard to the absorption spectrum of the clinical target, the corresponding reduction in possible destructive interference may permit a greater portion of the incident light available to transmit toward the target. For applications employing multiple successive laser pulses incident upon the same location, the temporally variable acoustic field displaces scatterers differently for successive laser pulses, which may also allow light to transmit to portions of the target missed by previous shots.

Another effect that is observed regards the shortening of the scattering wavevector in low pressure acoustic zones [185]. The same displacement that results in a decrease in the energy of the colliding photon and corresponds to the lower acoustic sidebands also reduces the magnitude of the optical wavevector following scattering events within low pressure zones. By contrast, in high pressure zones, the applied pressure incorporates the magnitude and directionality of the acoustic wavevector into the deflected optical wavevector [188]. This results in the generation of the higher acoustic sidebands, but also deflects the optical wavevector in the direction of the acoustic wavevector by transferring the mechanical energy of the displacement into 
the colliding photon. Extrapolating that assertion suggests that the applied pressure field skews the anisotropy of the tissue in the direction of the acoustic wavevector. The anisotropy of a turbid medium is defined to be the average cosine of the deflection angle following a scattering event, $g=<\cos (\theta)>$, which is therefore a value between -1.0 and 1.0, wherein a magnitude of 0 represents a perfectly isotropic medium and a magnitude of 1.0 represents a perfectly forward or backscattering medium [189]. With regard to biological tissue, anisotropy is typically between $0.8-1.0$, representing a largely forward-scattering medium, and is often considered to be around $g=0.90$ $[189,190]$. Consequently, if sonoillumination increased the forward anisotropy, there would be a corresponding increase in the likelihood that the clinical target would receive a greater dose of the applied light.

Each of the aforementioned effects are fundamentally tied to the properties of the applied pressure field, and are therefore strongly related to the magnitude, frequency, and propagation vector of the acoustic waveform. The impact of each property, however, is theorized to reach individual thresholds beyond which their influence either plateaus or begins to operate under distinctly different conditions. For example, with regard to acoustic magnitude, this threshold represents the maximum degree to which the tissue may be manipulated before necessitating a physiological change to the medium. In other words, the ultrasound does not remove the scatterers or absorbers, nor does it introduce any additional material into the medium or change its phase; accordingly, it can only modulate its properties so much before the modulation ceases to result in a measurable change in optical properties. Increasing the magnitude beyond this threshold likely results in minimal change in optical properties until such 
point as the overall structure of the medium is fundamentally altered, as occurs in sonophoresis through the ultrasonically-induced generation of hollow microcavities from dissolved gas [169].

Moreover, with regard to the propagation vectors, the theoretical threshold represents the distinction between the GRIF behaving less like a GRIN lens and more like a diffraction grating or GRIN optical fiber. Unlike traditional optical fibers that rely on internal reflection between core and cladding materials, GRIN optical fibers refract the light continuously within the cylindrical gradient index substrate, bending the light vector between the higher refractive index center and the lower refractive index exterior regions [191]. In tissue, the parallel regions of high and low refractive index could behave in a manner akin to GRIN fibers for sufficiently large values of $\theta_{A}$ at low frequency and thereby "guide" the light deeper into the tissue as if it were transmitting through a biological waveguide. This effect is anticipated to occur at lower frequencies $<1 \mathrm{MHz}$, as it will likely behave more like traditional UOT or A-O at higher frequencies. The theorized propagation vector threshold represents the angle between the optical and acoustic wavevectors, such that there is a transition between the GRIN lens region and the GRIN fiber region. Since the relative angle between the wavevectors is of greater importance than their direction with respect to the tissue, orthogonal incidence may be achieved by applying both light and acoustics at an angle to the skin or by refracting the acoustic wave into the tissue using an acoustic wedge. Literature review has not yielded evidence of any such behavior, which is tentatively attributed in part to the relative youth of ultrasonic modulation but is also suspected to be related to the high frequency nature of contemporary 
approaches, which seek to achieve diffractive effects or to modulate speckle, rather than to guide transmission.

Finally, based on the inverted relationship between acoustic pulsation frequency and the distance between successive pressure wavefronts (i.e. the acoustic wavelength), there will likely be a strong positive correlation between acoustic frequency and the likelihood that the sonoillumination effects will influence the propagation of any given photon. This relationship is based on the comparison of the acoustic wavelength for typical ultrasound in tissue, which for frequencies from $15 \mathrm{kHz}$ to $100 \mathrm{MHz}$ is between $10.63 \mathrm{~cm}$ and $15.95 \mu \mathrm{m}$ respectively (assuming $c_{s}=1595 \mathrm{~m} / \mathrm{s}$ ), with the optical transport mean free path length (TMFP) of the incident light within the tissue, which is typically around $1 \mathrm{~mm}$ for visible light $[174,192]$. The TMFP, also referred to as the "reduced mean free path," represents the average distance a photon will travel in tissue before losing "direction memory," after which it can be considered a randomly propagating photon, rather than part of an incident beam [190]. The TMFP can be calculated from the standard optical mean-free path length, which is the average distance a photon will travel between scattering events, as follows:

$$
\begin{aligned}
l_{m f p} & =\frac{1}{\mu_{s}} \\
\mu_{s}^{\prime} & =\mu_{s} *(1-g) \\
l_{m f p}^{\prime} & =\frac{1}{\mu_{s}^{\prime} *(1-g)} \\
\therefore \mathrm{TMFP} & =\frac{l_{m f p}}{(1-g)}
\end{aligned}
$$


wherein $\mu_{s}$ represents the scattering coefficient, $l_{m f p}$ is the mean free path length, $g$ is the anisotropy of the tissue, and $\mu_{s}^{\prime}$ is the reduced scattering coefficient 1 . As mentioned above, the photon loses direction memory after one TMFP into the tissue, which consequently often represents the limit for optical imaging techniques in tissue. However, since the typical TMFP for visible light is within the realm of available acoustic pulsation wavelengths, when the two are of comparable length, there is a much higher likelihood that the acoustic pulsation will influence the propagation of the photon. Assuming a TMFP of $1000 \mu \mathrm{m}$, the corresponding ultrasonic pulsation frequency is approximately $1.595 \mathrm{MHz}$. Based on these assertions, for frequencies less than $1.595 \mathrm{MHz}$, the impact of sonoillumination on photon migration will be largely due to modulation of refractive indices near the surface of the tissue; however, beyond this threshold frequency, each photon is increasingly likely to be impacted by the sonoillumination's effect on at least one collision event. Moreover, based on the same TMFP and the previously mentioned anisotropy of tissue, the typical MFP is on the order of $100 \mu \mathrm{m}$. As such, there may be a second threshold frequency at $15.95 \mathrm{MHz}$, since the distance between wavelengths would be comparable to the distance between individual scattering events, which represents a significant likelihood that the conditions of every collision event will have been altered by sonoillumination. Correspondingly, Table 4.1 includes the typical TMFP for a variety of biological tissues along with the corresponding acoustic frequency at the primary and secondary thresholds to anticipate the divisions of regimes based on tissue [174, 190]. 


\begin{tabular}{llll}
\hline \hline Tissue & TMFP & Primary threshold & Secondary threshold \\
\hline Muscle & $1.1 \mathrm{~mm}$ & $1.45 \mathrm{MHz}$ & $14.5 \mathrm{MHz}$ \\
Skin & $1.0 \mathrm{~mm}$ & $1.595 \mathrm{MHz}$ & $15.95 \mathrm{MHz}$ \\
Brain & $0.6 \mathrm{~mm}$ & $2.658 \mathrm{MHz}$ & $26.58 \mathrm{MHz}$ \\
Breast & $0.8 \mathrm{~mm}$ & $1.994 \mathrm{MHz}$ & $19.94 \mathrm{MHz}$ \\
Lung & $0.3 \mathrm{~mm}$ & $5.317 \mathrm{MHz}$ & $53.17 \mathrm{MHz}$ \\
\hline \hline
\end{tabular}

TABLE 4.1 Typical TMFP in various tissues listed with corresponding acoustic frequencies at the primary threshold, wherein the wavelength corresponds to the TMFP, and the secondary threshold, wherein the wavelength corresponds to the MFP $[174,190]$.

\subsection{Materials and methods}

Although the amplitude, frequency, and propagation angle are each likely to have significant influence on the method and efficacy of sonoillumination, due to the limitations of the equipment available, the experiments performed herein exclusively investigated the effect of varying the magnitude of acoustic pulsation on optical transmission. The following sections describe the procedures involved in waveguide fabrication and tissue sample preparation, along with a description of the optical apparatus employed in the associated experiments. Additionally, details regarding the ultrasonic pulsation electronics are also included.

\subsubsection{Porcine tissue samples}

Large sections of ex vivo porcine skin were donated for this research project from the Mizzou Meat Market (College of Agriculture, Food, and Natural Resources at the University of Missouri). The skin was disinfected in a $1.75 \%$ ethanol solution for 2 minutes prior to processing for experimentation. Following disinfection, the sections were trimmed down to a thickness of $4 \mathrm{~mm}$, incorporating epidermal, dermal, and 


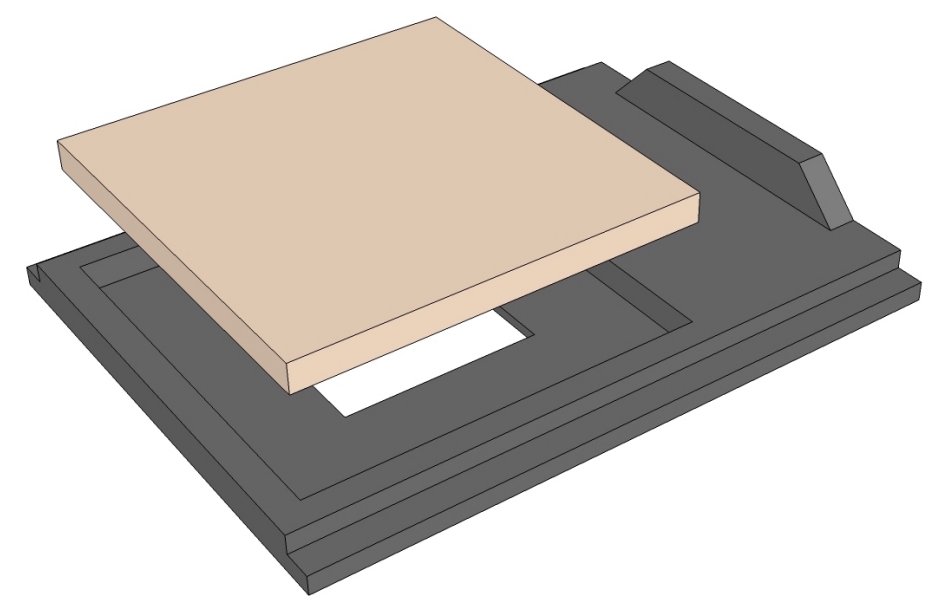

FigURE 4.4 3D printed cartridges were designed to keep the porcine skin taut during experimentation while also allowing for consistent and easy interchanging of samples.

adipose tissue, and cut into $40 \mathrm{~mm}$ square samples. Originally, each sample was held taut and adhered at the edges with superglue over a thin fiberglass mesh to individual 3D-printed tissue mounts, as shown in Figure 4.4, which incorporated open apertures to allow for measurement of optical transmission through the tissue. The fiberglass mesh was included to provide additional support to the porcine skin and prevent potential indentation of the tissue during testing. Processed skin samples were wrapped in moist Kim wipes to prevent dehydration, enclosed in air-tight plastic bags, and stored in a freezer at $4^{\circ} \mathrm{C}$ until needed. Prior to use, samples were defrosted by running water over the plastic bag for $10-15 \mathrm{~min}$.

\subsubsection{Waveguide fabrication}

The optical waveguides were fabricated in-house from a large sheet of $1.5 \mathrm{~mm}$ thick polymethyl-methacrylate (PMMA) acrylic glass (Evonik CRYO, 0A000 GT). Waveguide substrates were cut into $28 \times 25.5 \mathrm{~mm}$ units and machined to incorporate 45 angled faces at two opposing ends, as shown in Figure 4.5. Both of the angled 


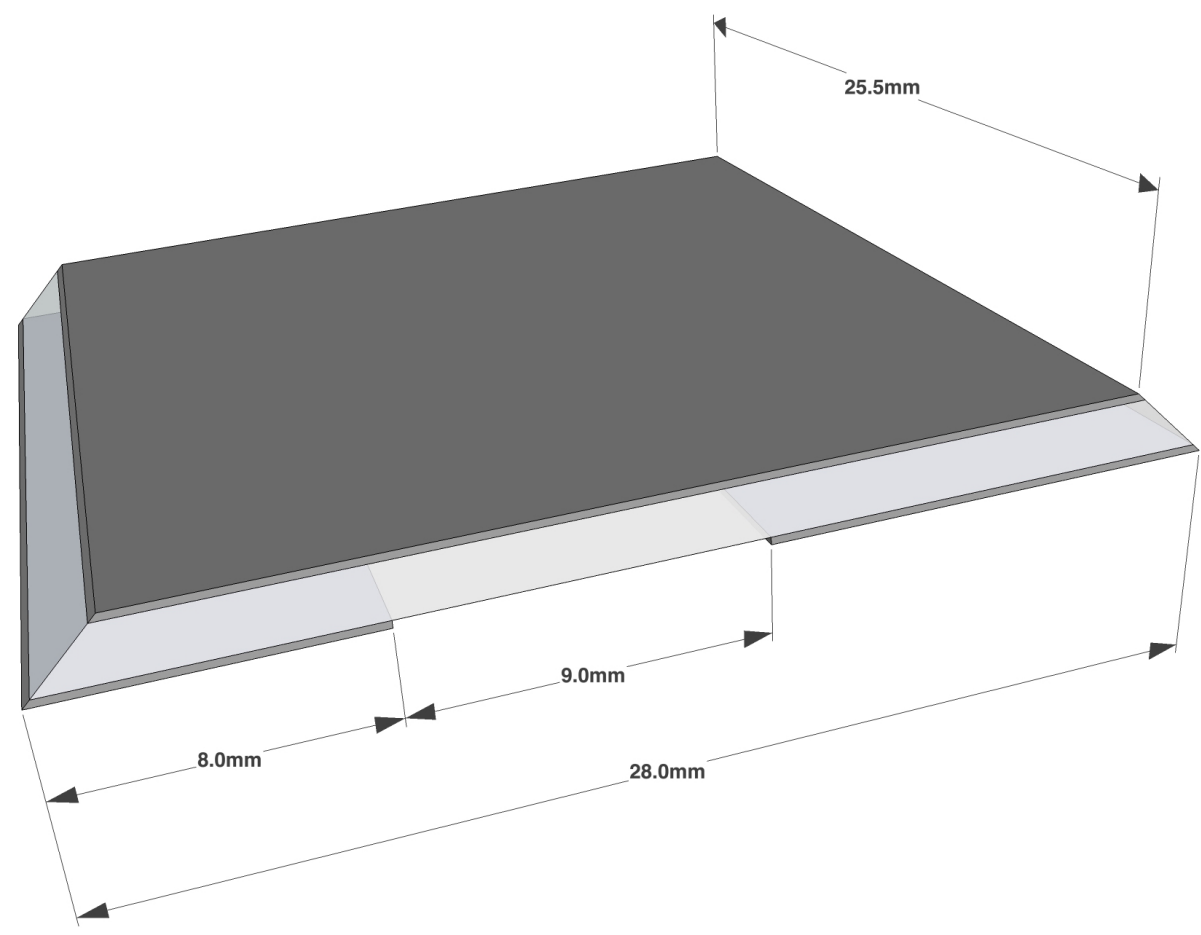

FiguRE 4.5 A planar optical waveguide composed of a PMMA substrate clad in thin films of silver ensured internal reflection angles of $45^{\circ}$ and enabled the transmission of laser light into porcine tissue through direct physical contact with the exposed "active area" on the waveguide's largest face.

faces and the two largest faces were polished using optical sanding paper with 200, 800, 1000, and 2000 grits in increasing order, followed by a final polish using a fine scratch removing compound (Novus Fine Scratch Remover No. 2). The two angled entrance and exit apertures of the substrates were masked off with Kapton tape, along with a $9 \mathrm{~mm} \times 25.5 \mathrm{~mm}$ rectangular section of the largest face, which would serve as the waveguide's active area. The substrates were then clad in thin films of titanium and silver using a cold sputtering vacuum deposition system (Quorum Technologies, K575X) to serve as mirrors on the bounding surfaces of the substrate. The titanium was deposited using a $99.998 \%$ pure Ti target at $90 \mathrm{~mA}$ for $60 \mathrm{~s}$, which formed a film less than $10 \mathrm{~nm}$ thick. The titanium acted as an adhesion layer for the silver, which 


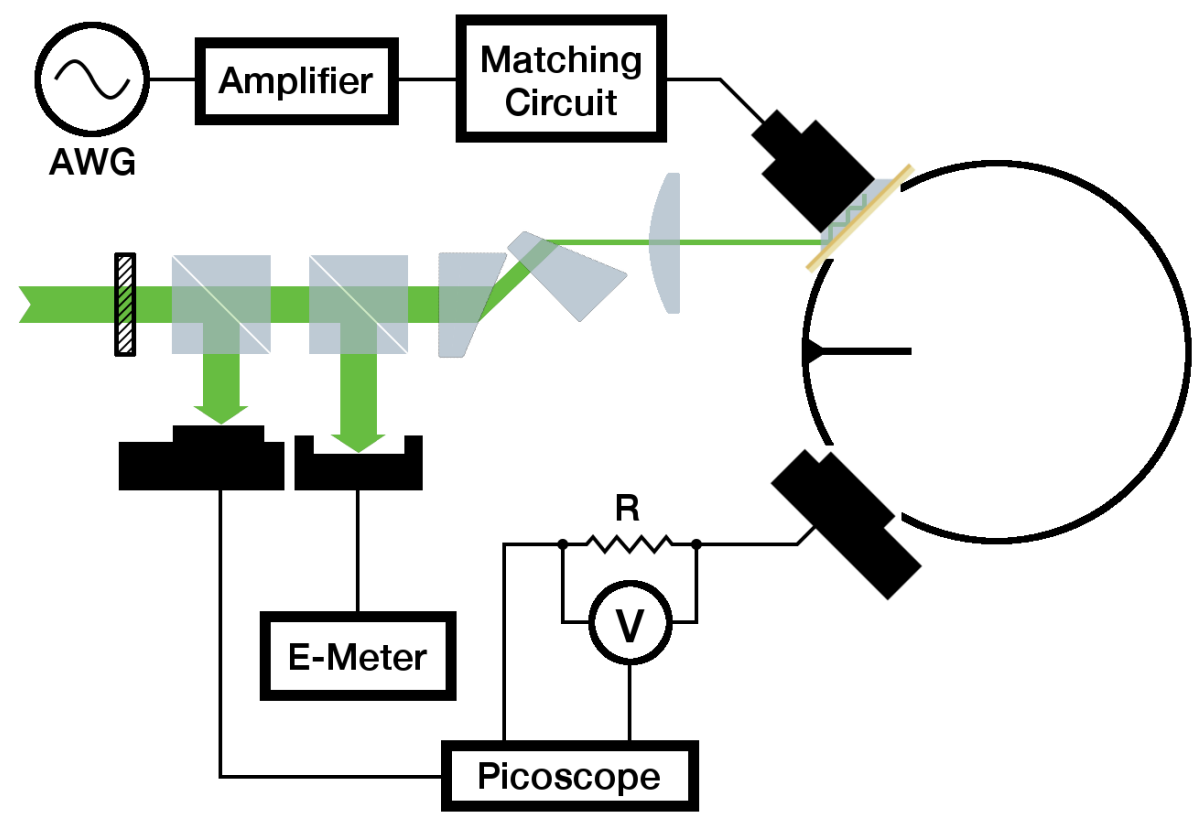

FIGURE 4.6 Laser light at $532 \mathrm{~nm}$ triggered data collection, was measured for pulse-topulse energy variations, and was then transmitted through porcine skin into an integrating sphere by a planar waveguide. Ultrasound was applied to the rear face of the waveguide, driven by an AWG, amplified $50 \mathrm{~dB}$, and impedance matched to improve electrical transmission. Light was detected by a reverse-biased photodiode, whose photovoltage spike was measured by a voltage probe across a series resistor, $\mathrm{R}$.

was deposited from a $99.95 \%$ pure Ag target at $30 \mathrm{~mA}$ for $300 \mathrm{~s}$ to form a much thicker layer approximately $200 \mathrm{~nm}$ thick. Laser light incident perpendicularly upon one of the angled faces would internally reflect along the waveguide to the exit aperture when exposed to air, but would transmit out through the active area when in contact with tissue.

\subsubsection{Experimental apparatus}

The goal of these experiments was to investigate the potential for increased light fluence through epidermal, dermal, and adipose tissues under continuous ultrasonic pulsation with relevance to potential clinical applications in laser dermatology. Accordingly, pulsed laser light, generated by a Q-switched Nd:YAG laser (Opotek, 
Vibrant $355 \mathrm{II})$ operated at a wavelength of $532 \mathrm{~nm}$ with a $5 \mathrm{~ns}$ pulse width, was transmitted through porcine skin samples and measured using a reverse-biased $\mathrm{Si}$ photodiode (Thorlabs, DET100A) attached to an integrating sphere. The light was guided into the tissue using silvered optical waveguides following a short optical train that modified the beam shape to better suit a planar waveguide and directed light toward a second photodiode to trigger data collection and a power meter to account for variations in pulse-to-pulse laser intensity. The optical train, shown in Figure 4.6, consisted of a zero-order half-waveplate (Thorlabs, WPH05M-532) and polarizing beamsplitter cube (Thorlabs, CM1-PBS251) to direct a small portion of light toward the trigger photodiode (Thorlabs, DET10A), while transmitting the remaining s-polarized light along the length of the train. A standard 50:50 beamsplitter cube was used to direct half of the beam intensity toward a power meter (Thorlabs, PM100USB) and the remaining half toward a 4X anamorphic prism pair (Thorlabs, PS883-A), which compressed the beam along one axis to transform the incident circular beam into an elliptical Gaussian profile approximately $1.5 \mathrm{~mm}$ thick along its narrow axis. To compensate for the dispersive effects caused by the extreme degree of compression, a long focus cylindrical lens (Thorlabs, LJ1558L2-A) re-collimated the beam prior to entering the waveguide.

The waveguides were fixed at a $45^{\circ}$ incline with respect to the incident beam using a custom 3D printed optomechanical mount (Figure 4.7) that rigidly connected the optical train to the integrating sphere while allowing for interchangeable tissue samples and waveguide cartridges. At this incline, the incident light was perpendicular to the angled face of the substrate, which minimized refraction and ensured that 


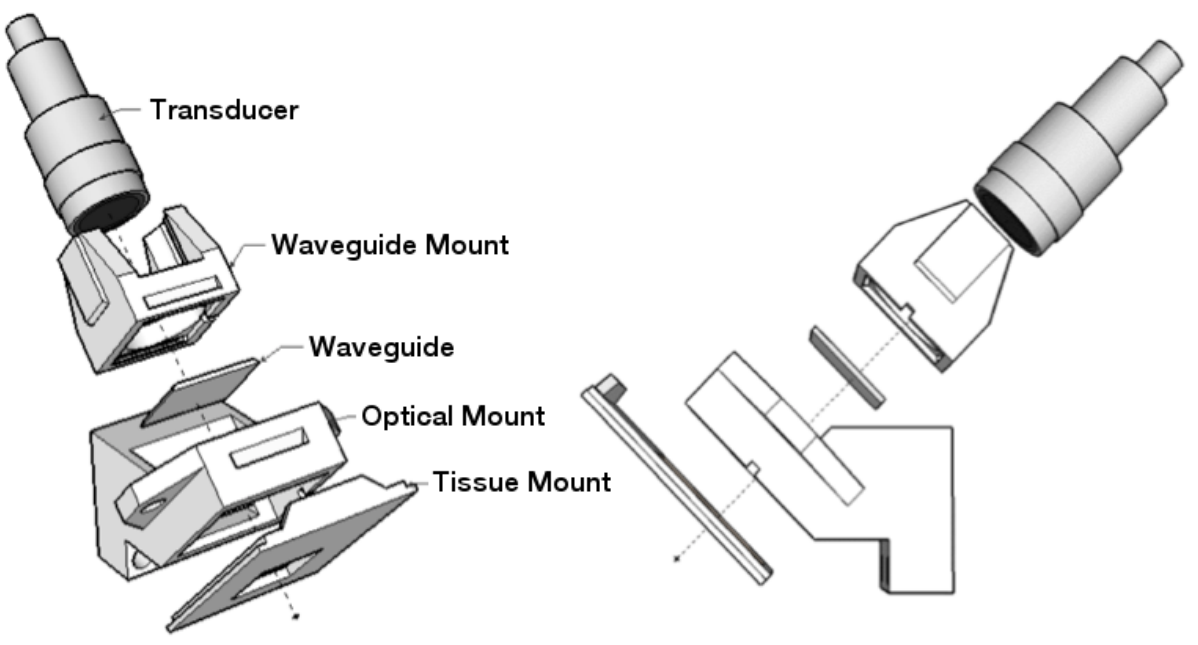

FigURE 4.7 The waveguides were sealed into 3D printed mounts to ensure consistent alignment between tests while permitting the ultrasound transducer to be coupled to the rear face. The waveguide cartridges were held in place against the tissue samples using additional 3D printed optomechanics.

the beam within the waveguide was also internally reflecting at $45^{\circ}$. Figure 4.7 also depicts the 3D printed waveguide cartridges, which allowed for the incorporation of an ultrasonic transducer operated at a central frequency of $510 \mathrm{kHz}$. When assembled, the transducer was in direct contact with the rear face of the waveguide whereas the waveguide active area was in direct contact with the porcine tissue sample. To ensure sufficient optical and ultrasonic transduction, a thin layer of Cargille Type NVH immersion oil was applied between each interface.

A combination USB data acquisition device (DAQ) and arbitrary waveform generator (AWG) (Picoscope, 5442B) was used for both data collection and ultrasonic pulse generation. The AWG output a sine wave at $510 \mathrm{kHz}$, whose amplitude was computationally controlled. The signal was then amplified by a $50 \mathrm{~dB}$ gain high frequency RF Amplifier (Electronics \& Innovation, 350L) and transmitted via BNC cable to an L-match electrical impedance matching circuit, which then passed the 
signal along to the transducer for acoustic pulse generation. The impedance matching circuit minimized electrical reflections within the BNC cable to maximize transmission of power to the transducer.

\subsubsection{Ultrasonic Pulsation}

In addition to recording the optical transmission data, the Picoscope $5442 \mathrm{~B}$ oscilloscope also incorporated an arbitrary waveform generator (AWG). The AWG was used to output a sine wave at the central frequency of each transducer. The waveform was transmitted through a $50 \Omega$ BNC cable into a $50 \mathrm{~W}$ RF Amplifier (Electronics \& Innovation 350L), which was capable of amplifying the input waveform amplitude by $50 \mathrm{~dB}$ at frequencies ranging from $250 \mathrm{kHz}-150 \mathrm{MHz}$.

The transducers used in these experiments incorporated piezoelectric elements to generate the ultrasound pulses. Although the output electrical impedance of the RF amplifier was set to $50 \Omega$, the input impedance of the transducers was inversely frequency dependent, increasing substantially with decreasing central frequency. In order to achieve sufficient power transmission from the amplifier to the transducers, electrical impedance matching circuits were designed for each transducer considered. Only one transducer was used for this study, chosen based on experimentally determined capabilities balancing thermal stability under high electrical power with the availability of impedance matching components. In order to properly match the transducers, the electrical impedance spectrum of each transducer was recorded using two 10X voltage probes. The transducers were each put in series with a $100 \Omega$ thin film resistor, assuming the impedance of the resistor to be predominantly resistive 


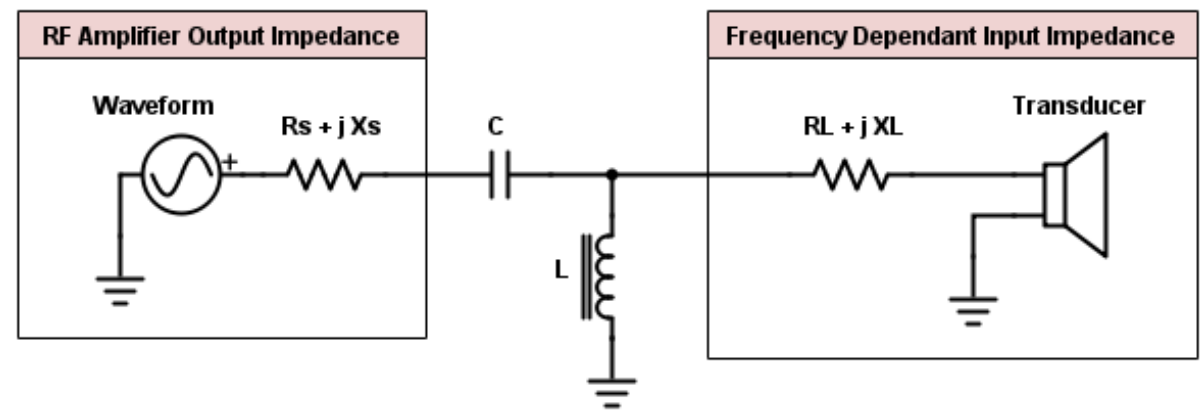

FIGURE 4.8 An L-match circuit, consisting of a capacitor in series with the driving pulse and an inductor shunted to ground in parallel with the transducer, was used to more closely match the electrical impedances of the BNC cable and the ultrasonic transducer.

(as opposed to reactive) in the frequency regimes used. A positive square pulse was transmitted through these simple circuits, with pulse width set according to the center frequency of the transducer. Matlab Fast Fourier Transform (FFT) analysis was performed in order to generate a frequency spectrum for the real valued resistance and complex valued reactance of each transducer, and thereby calculate its impedance at its center frequency.

To more closely match the impedance of the transducer to the $50 \Omega$ output impedance from the RF amplifier, a high-pass, DC-blocking "L-match" L-C impedance matching circuit was designed, as is shown in Figure 4.8. The general design of an L-match circuit consists of a capacitor, $C$, in series with the transducer and an inductor, $L$, shunted to ground in parallel with the transducer [193]. The name "L-match" is derived from the shape of the circuit; there are also T-match and pi-match circuits, which vary based on the positioning of each component with respect to the load and ground. The circuits were designed to more closely match the effective impedance of the transducer to the $50 \Omega$ impedance of the $\mathrm{RF}$ amplifier and $\mathrm{BNC}$ cables, to permit adequate signal transmission to the transducer. 
The transducer was press-fit into a 3D printed cartridge, which incorporated a thin space between the transducer active face and the rear face of the waveguide. This space was filled with Cargille Type NVH oil to ensure proper transmission into the waveguide. Experiments had originally called for the use of standard ultrasound gel, such as is used in clinical applications; however, the gel exhibited significant problems with power transmission into external media through the waveguide, possibly due to an acoustic impedance mismatch between the gel and the waveguide substrate. The transducers used were immersion transducers, and as such were acoustically impedance-matched to water. The acrylic waveguide substrates were chosen as they would exhibit a relatively low impedance mismatch with water, tissue, and oil, which are all characterized as having comparable acoustic impedances.

\subsubsection{Experimental procedures}

During experimentation, the data collection was performed by a custom designed LabVIEW automated program, which was designed to control laser firing, ultrasonic driving amplitude, laser energy measurement, triggering, and data collection. The laser was controlled via an RS-232 cable connected to the computer and operated using standard serial communication commands, as outlined in the Brilliant Laser manual. The program was designed to output a sinusoidal waveform from the Picoscope's AWG port, which would be connected via BNC cable to the RF amplifier, followed by the impedance matching circuit and transducer. The amplitude of the waveform was user-defined and could be set within the Picoscope's maximum range of $0 i V_{R M S} 2 \mathrm{~V}$. During experimentation, the program would enable the waveform 
output for $10 \mathrm{~s}$ prior to laser irradiation, after which period the laser was pulsed in single-fire mode at roughly $1 \mathrm{~Hz}$. Detection of the laser pulse by the photodiode would trigger data collection by the Picoscope. The software would then record the peak amplitudes of the photovoltage from the integrating sphere photodiode and the corresponding photocurrent, by measuring the voltage drop across a $50 \Omega$ series resistor with a $1 \mathrm{X}$ voltage probe. The program repeated this process for 200 consecutive shots and exported the file as a comma separated value file (*.csv). The Matlab code included in Appendix $\mathrm{C}$ was used to analyze the data and account for pulse to pulse laser energy variations. Data was recorded for ultrasound driving amplitudes from 0 to $700 \mathrm{mV}$ in $100 \mathrm{mV}$ increments.

\subsection{Results}

The following sections present the results of the transducer electrical impedance spectral measurements followed by optical transmission data for light under various ultrasonic pulsation energies. A brief discussion of the mathematical analysis of the impedance data is also included for the reader's convenience.

\subsubsection{Electrical Impedance Measurements}

As mentioned in the previous section, the electrical impedance frequency spectrum was calculated for each transducer under consideration. This was performed by recording the time-resolved voltages across a $100 \Omega$ thin film resistor placed in series with each transducer [194]. The voltages were recorded using 10X oscilloscope voltage probes on Channels $\mathrm{A}$ and $\mathrm{B}$ of the Picoscope, with the output square pulse also 
generated from the AWG port of the Picoscope. The pulse was a $2 \mathrm{~V}$ positive square pulse 256 data points long followed by 16128 points at baseline to temporally separate pulses, such that only a single pulse would be recorded within the window of interest. In order to match the 256 point wide pulse to the appropriate temporal pulse width for each transducer, the AWG was operated at various frequencies, calculated by the expression:

$$
f_{\text {operation }}=\frac{N_{\text {pulse }}}{d t * N_{\text {total }}}
$$

wherein $d t$ represents the period of the transducer at its center frequency and $N_{\text {pulse }}$ is the unitless length of the pulse in data points and $N_{\text {total }}$ is the total number of data points recorded, which for the purposes of this experiment were 256 and 16384 points respectively. Matlab was then used to calculate the $N$-point discrete Fourier transform (DFT) of a single pulse recorded on each channel, where $N$ was determined to be the nearest power of 2 to the total number of recorded data points, amending any under-filled FFTs with zeros. The impedance spectrum of the transducer was then calculated using the following equation:

$$
Z_{\text {Transducer }}=\frac{F\left\{C h_{B}(t)\right\}}{F\left\{C h_{A}(t)\right\}-F\left\{C h_{B}(t)\right\}} Z_{\text {Resistor }}
$$

where $F\left\{C h_{A}(t)\right\}$ and $F\left\{C h_{B}(t)\right\}$ are the discrete Fourier transforms of the time domain voltage traces from the probe attached before the resistor, plugged into Channel A, and that from the probe attached immediately following the resistor, plugged into 
Channel B. The complex impedance of the transducer was then calculated to be:

$$
\begin{aligned}
Z_{\text {Load }} & =R_{L}+j X_{L} \\
\text { wherein: } R_{L} & =\left|Z_{\text {Transducer }}\right| \cos \left(\angle Z_{\text {Transducer }}\right) \\
X_{L} & =\left|Z_{\text {Transducer }}\right| \sin \left(\angle Z_{\text {Transducer }}\right)
\end{aligned}
$$

The equivalent circuit of a piezoelectric transducer can be represented by a capacitor in parallel with a resistor, capacitor, and inductor all in series, wherein the resistor accounts for the real valued load resistance, $R_{L}$, and the remaining components account for the complex valued load reactance, $X_{L}$ [195]. Figure 4.9 shows the resistance and reactance of a $500 \mathrm{kHz}$ transducer. The Matlab code used to analyze the time-resolved voltage data from the two voltage probes and generate the impedance spectra included herein is as follows:

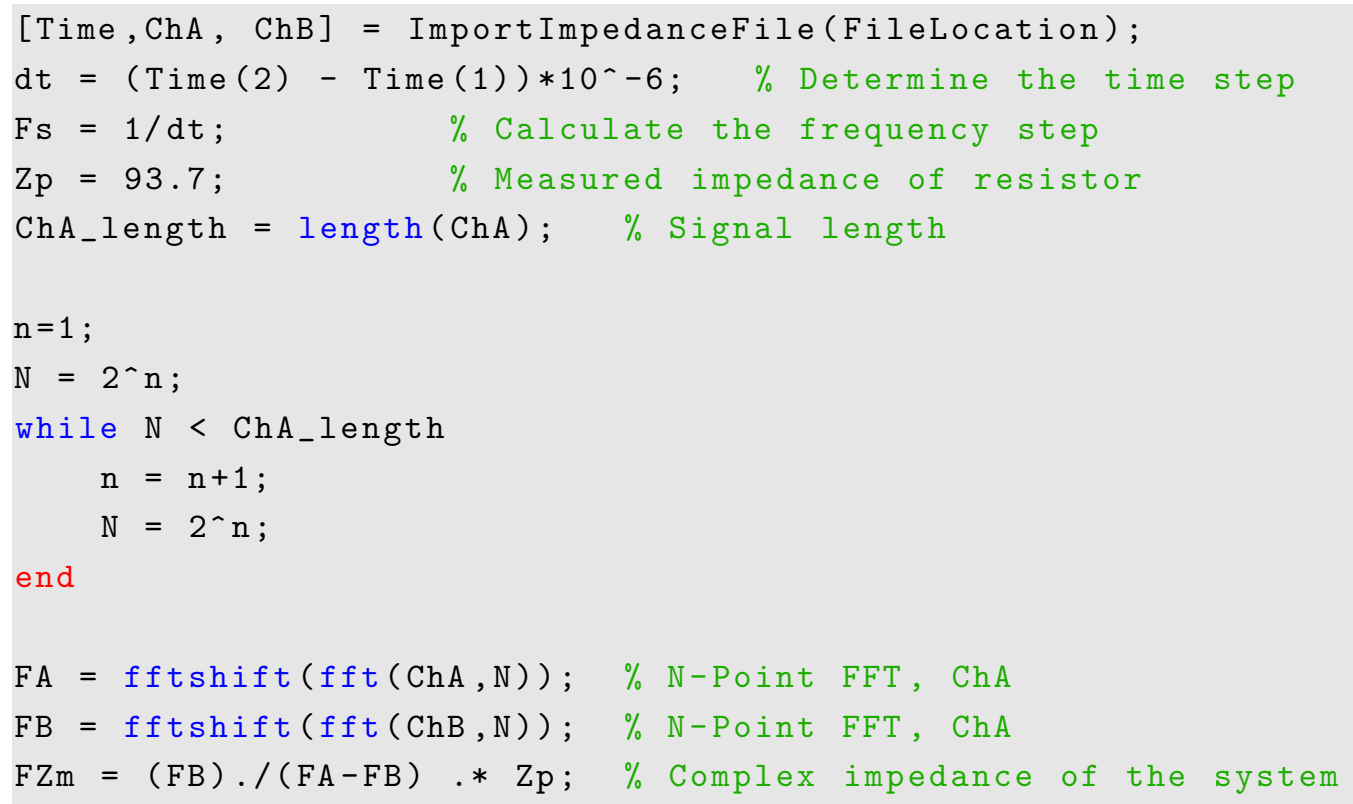




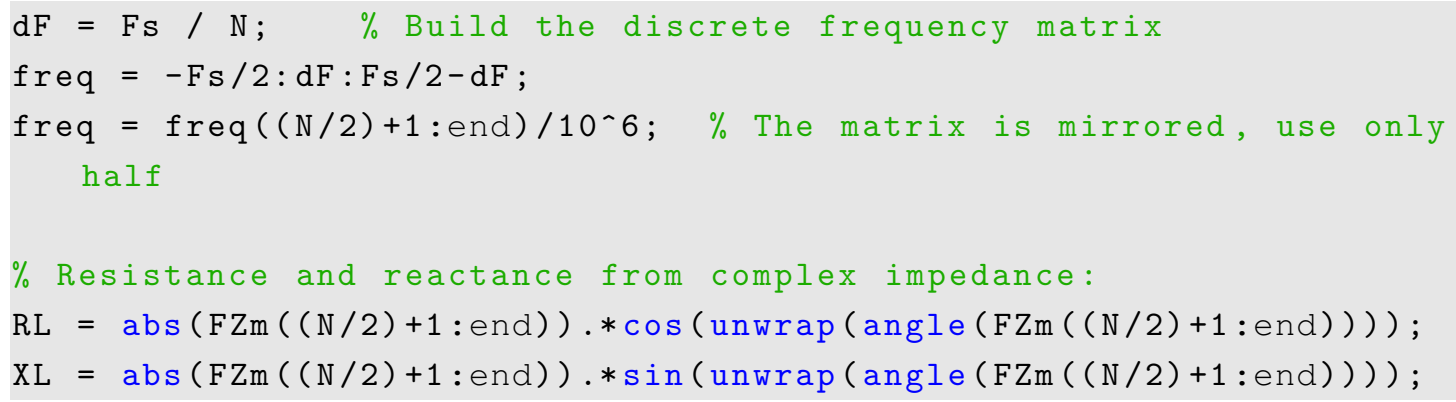

Brief experimentation determined that the higher frequency transducers were subject to thermal destabilization at greater amplitudes, in some cases resulting in the catastrophic failure of the piezoelectric element or delamination of the piezoelectric from the acoustic impedance matching layers installed by the manufacturer. Accordingly, the $500 \mathrm{kHz}$ transducer was determined to be the most appropriate for continued testing, as it withstood the highest amplitudes while maintaining proper functionality; although, it may be possible to utilize different waveforms or duty cycles to avoid overloading the higher frequency transducers. Alternatively, it may be possible to actively cool the transducer casing or utilize transformer-based impedance matching circuits to improve high frequency operation.

At the center frequency of the $500 \mathrm{kHz}$ transducer, the total impedance was found to be $Z_{\text {Load }}=89.01-j 630.73$, which necessitated the construction of an impedance matching circuit. The load impedance measured for each transducer prior to the design of impedance matching circuits is included in Table 4.2. The circuit for the $500 \mathrm{kHz}$ transducer was designed to utilize $C=657 \mathrm{pF}$ and $L=85.8 \mu \mathrm{H}$; however, due to limited availability of components with sufficient power dissipation capabilities, the single inductor $L$ was replaced with five inductors consisting of an $82 \mu \mathrm{H}$ inductor in series with an additional four $1.05 \mu \mathrm{H}$ inductors. As a consequence of this design 

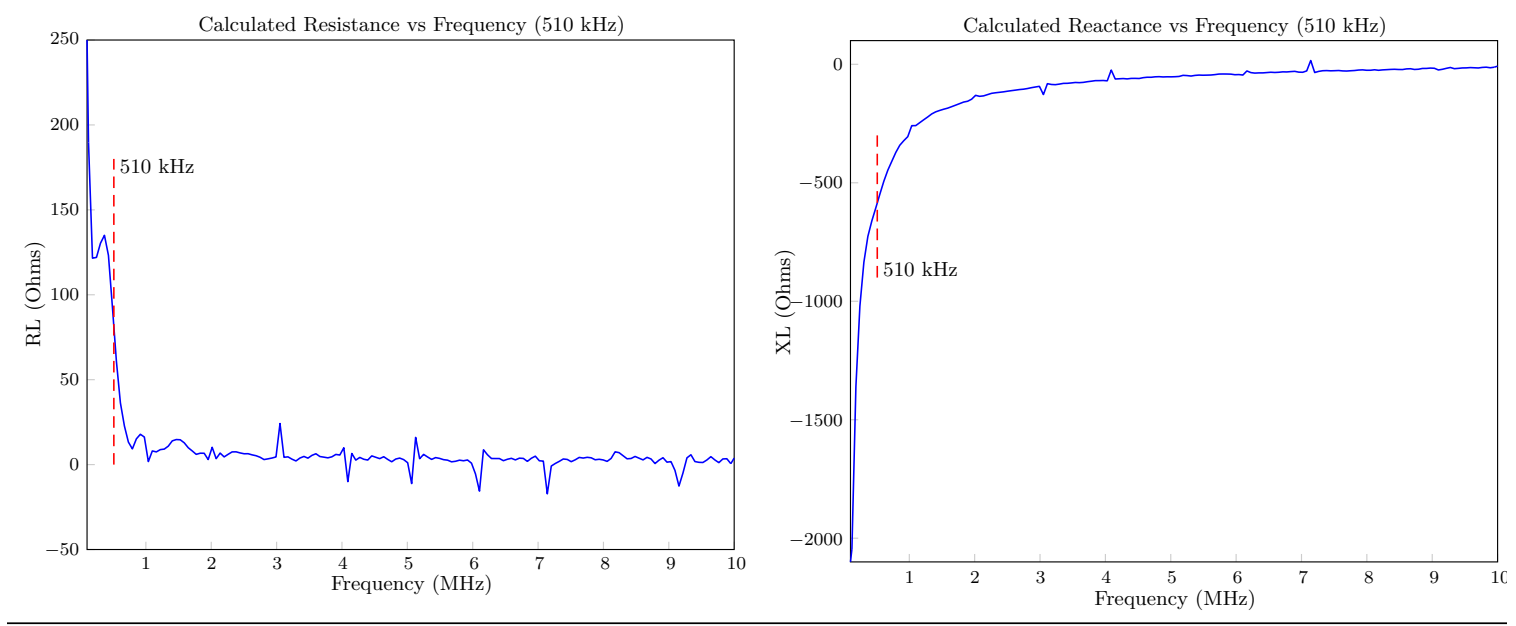

FigURE 4.9 The resistive and reactive components of the complex impedance spectrum as measured for the $500 \mathrm{kHz}$ transducer. The intermittent spikes are artifacts caused by the $100 \Omega$ thin film resistor.

\begin{tabular}{llllll}
\hline \hline$f_{\text {nominal }}$ & $f_{\text {center }}$ & $f_{\text {operation }}$ & $Z=R_{L}+j X_{L}$ & $|Z|(\Omega)$ & $\Gamma_{S L}$ \\
\hline $500 \mathrm{kHz}$ & $510 \mathrm{kHz}$ & $15.94 \mathrm{kHz}$ & $Z=89.01-j 630.73$ & 636.98 & 0.8544 \\
$1.0 \mathrm{MHz}$ & $1.0 \mathrm{MHz}$ & $31.25 \mathrm{kHz}$ & $Z=95.47-j 409.9$ & 420.87 & 0.7876 \\
$5.0 \mathrm{MHz}$ & $5.24 \mathrm{MHz}$ & $163.78 \mathrm{kHz}$ & $Z=8.06-j 21.89$ & 23.33 & -0.3637 \\
$7.5 \mathrm{MHz}$ & $7.55 \mathrm{MHz}$ & $236.03 \mathrm{kHz}$ & $Z=10.81-j 22.44$ & 24.91 & -0.3349 \\
$10.0 \mathrm{MHz}$ & $10.69 \mathrm{MHz}$ & $333.87 \mathrm{kHz}$ & $Z=16.36+j 2.92$ & 16.61 & -0.5013 \\
\hline \hline
\end{tabular}

TABLE 4.2 Measured complex electrical impedance values for each transducer employed in this study, along with calculated total impedance, $|Z|$, and source-load reflection coefficient, $\Gamma_{S L}$. Negative reflection coefficients are indicative of a $180^{\circ}$ phase change in the reflected waveform. The operating frequency, $f_{\text {operation }}$, for impedance measurements is also included, based on the specific square pulse that was used.

concession, the capacitor was increased to $680 \mathrm{pF}$, which was determined experimentally to provide better matching between source and load. Particular care should be taken that all components in such circuits are capable of withstanding the voltages, currents, and powers that they are expected to encounter, accounting for power dissipation across inductors using heatsinks and active cooling fans as necessary. 


\subsubsection{Relationship between photocurrent and laser energy}

In order to determine what, if any, correlation existed between the amplitude of ultrasonic pulsation and the degree of transdermal transmission, successive experiments were conducted under increasing ultrasonic driving amplitudes. Prior experimentation revealed that the voltage spikes produced by the laser impacting the photodiode detection surface were logarithmically related to the transmitted optical power. However, technical documents from the photodiode manufacturer suggested that measuring the photocurrent as opposed to the photovoltage would yield a more linear relationship to the incident laser energy. Lacking access to a current probe, a voltage probe was used to measure the voltage drop across a series resistor of known resistance to calculate the photocurrent based on Ohm's Law, Eq. 4.14, wherein the current is equivalent to the change in voltage divided by the resistance.

$$
I=\frac{\Delta V}{R}
$$

Laser light was transmitted into the integrating sphere, varying the laser's QSwitch delay to control output intensity. The effective photocurrent (i.e. photovoltage drop divided by $50 \Omega$ ) was recorded for each individual shot along with the output laser intensity of the beam directed toward the energy meter shown in Figure 4.6. The data for this series of tests is shown in Figure 4.10, showing the relationship between photocurrent and laser energy. Although the technical documents had specified that the relationship would be linear, a brief observation of the full dataset suggested 


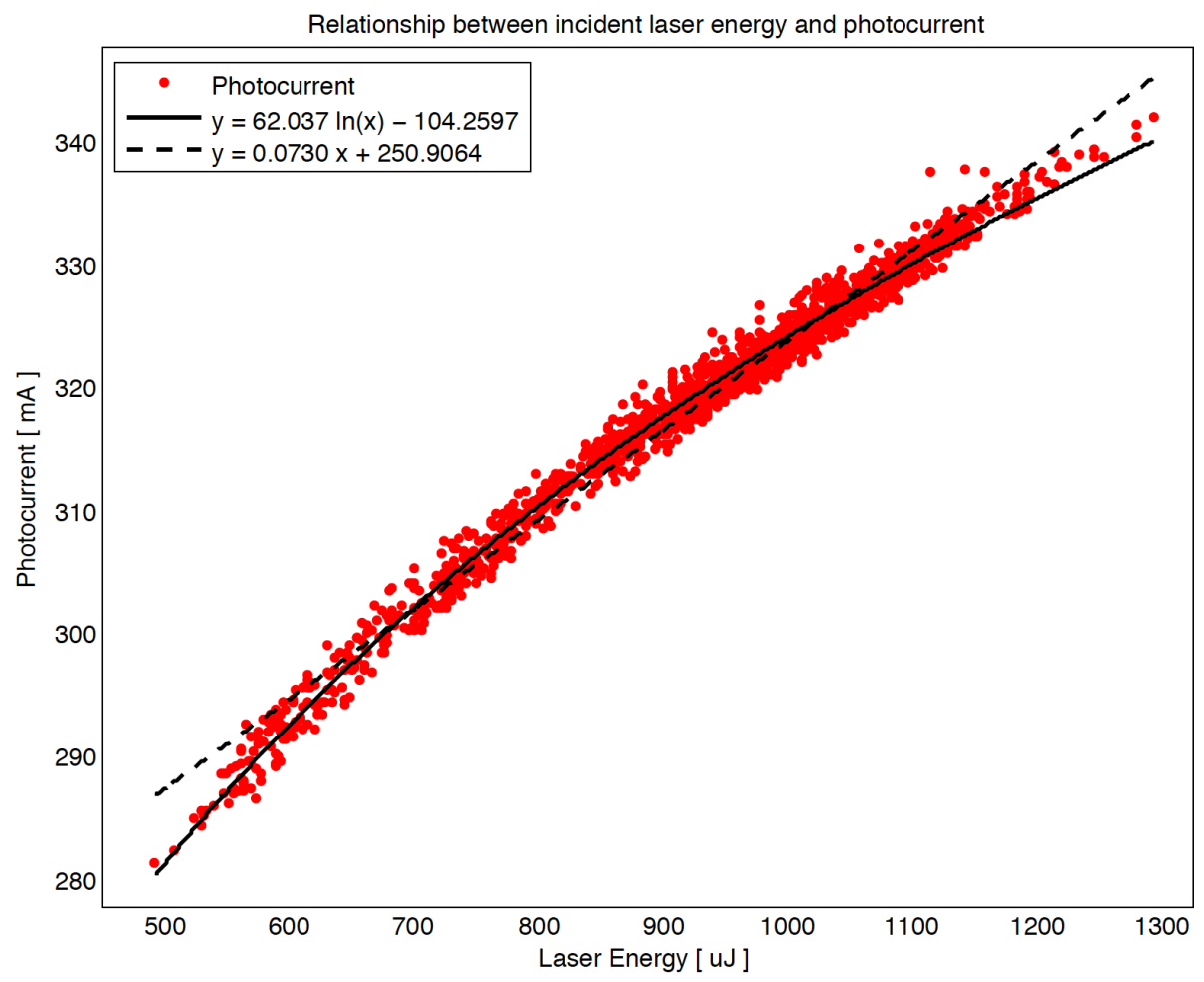

FIGURE 4.10 Comparison of least-squares regressions revealed that photocurrent was logarithmically related to incident laser energy, which indicated that the current needed to be converted into units of optical power prior to evaluating any potential improvement in transmission.

that the relationship might still be somewhat logarithmic. Accordingly, the data was fit with both linear and nonlinear logarithmic regressions, which were compared for accuracy and reliability of fit. The linear regression was of the form $y=\beta_{1} x+$ $\beta_{0}$, wherein the independent variable $x$ represents the incident laser energy and the dependent variable $y$ represents the photocurrent; similarly, the logarithmic regression was of the form $y=\beta_{1} \ln (x)+\beta_{0}$. The regression coefficients for each fit are shown in Table 4.3, accompanied by their Mean Squared Error (MSE) and coefficients of determination $\left(R^{2}\right)$. 


\begin{tabular}{lllll}
\hline \hline Regression & $\beta_{1}$ & $\beta_{0}$ & MSE & $\mathbf{R}^{2}$ \\
\hline Linear & 0.07304 & 250.9064 & 2.3104 & 0.9820 \\
Logarithmic & 62.0368 & -104.2598 & 1.5650 & 0.9878 \\
\hline \hline
\end{tabular}

TABLE 4.3 Regression coefficients and statistics relating photocurrent to laser energy.

\subsubsection{Optical transmission enhancement}

After establishing the relationship between laser energy and photocurrent, transdermal transmission tests were performed under ultrasonic pulsation of varying intensity. The ultrasound intensity was controlled via a software-driven arbitrary waveform generator, which output sine waves at specified voltage amplitudes. Driving amplitudes from $100 \mathrm{mV}$ to $700 \mathrm{mV}$ were tested in $100 \mathrm{mV}$ increments. For the purposes of these experiments the control data was recorded under identical transmission conditions, but with a driving voltage of $0 \mathrm{mV}$. The output sine wave was amplified $50 \mathrm{~dB}$ before transduction into an ultrasonic pulse. The light was transmitted via optical waveguide through a sample of $4 \mathrm{~mm}$ thick porcine skin with tests performed in quick succession in order of increasing amplitude. In each case, the ultrasound was inactivated between each test and engaged for 10 seconds prior to light transmission at the beginning of each test.

Figure 4.11 depicts the final, post-analysis data recorded from each test, grouped by ultrasonic driving amplitude. For each test, 200 data points were recorded; however, to avoid measurement bias at the start and end of each test series, 5 points were discarded at each end, owing to inconsistencies in photocurrent measurement and laser output energies during those regimes. Having previously recorded intermittent background noise in the system from nearby electrical devices, the data was cleaned 


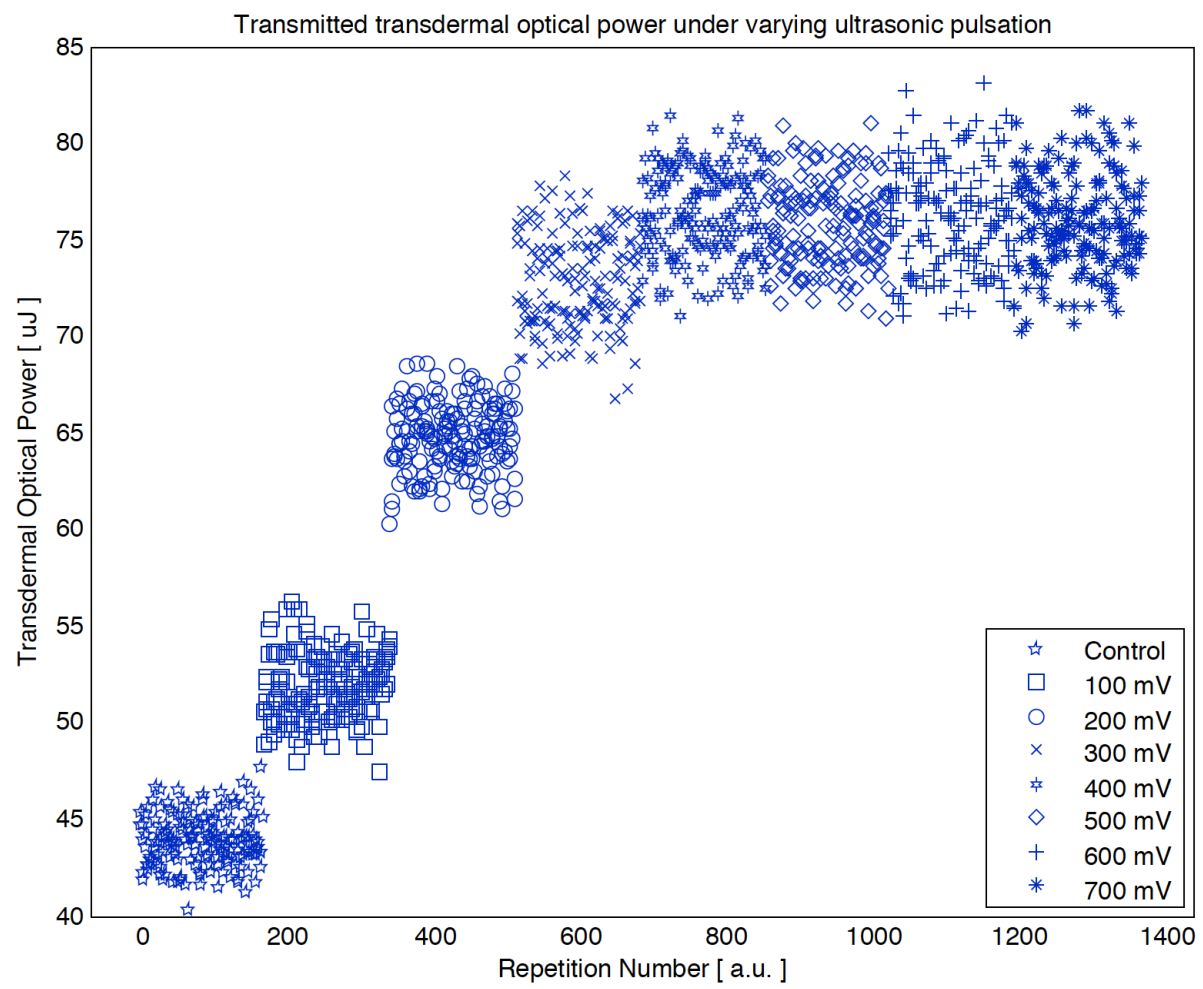

FIGURE 4.11 The application of ultrasonic pulsation yielded distinct differences in measured optical power with increasing driving amplitude until leveling off above $400 \mathrm{mV}$, suggesting that there is an upper limit to the degree of tissue modulation that occurs under low intensity pulsation.

for outliers, which occurred roughly every 12 th data point, by identifying outlier indices using normalized datasets. The normalized datasets were generated according to Eq. 4.15, wherein $P(x)$ represents the photocurrent at index $x$, whereas $\mu_{n}$ and $\sigma_{n}$ represent the relative means and standard deviations of the respective test.

$$
N(x)=\frac{P(x)-\mu_{n}}{\sigma_{n}}
$$

Outliers were defined as those points that occurred beyond the $90 \%$ confidence interval established by $\mu_{\text {norm }} \pm 1.645 \sigma_{\text {norm }}$, wherein $\mu_{\text {norm }}$ and $\sigma_{\text {norm }}$ represent the mean and standard deviation of the respective normalized datasets. Additionally, 


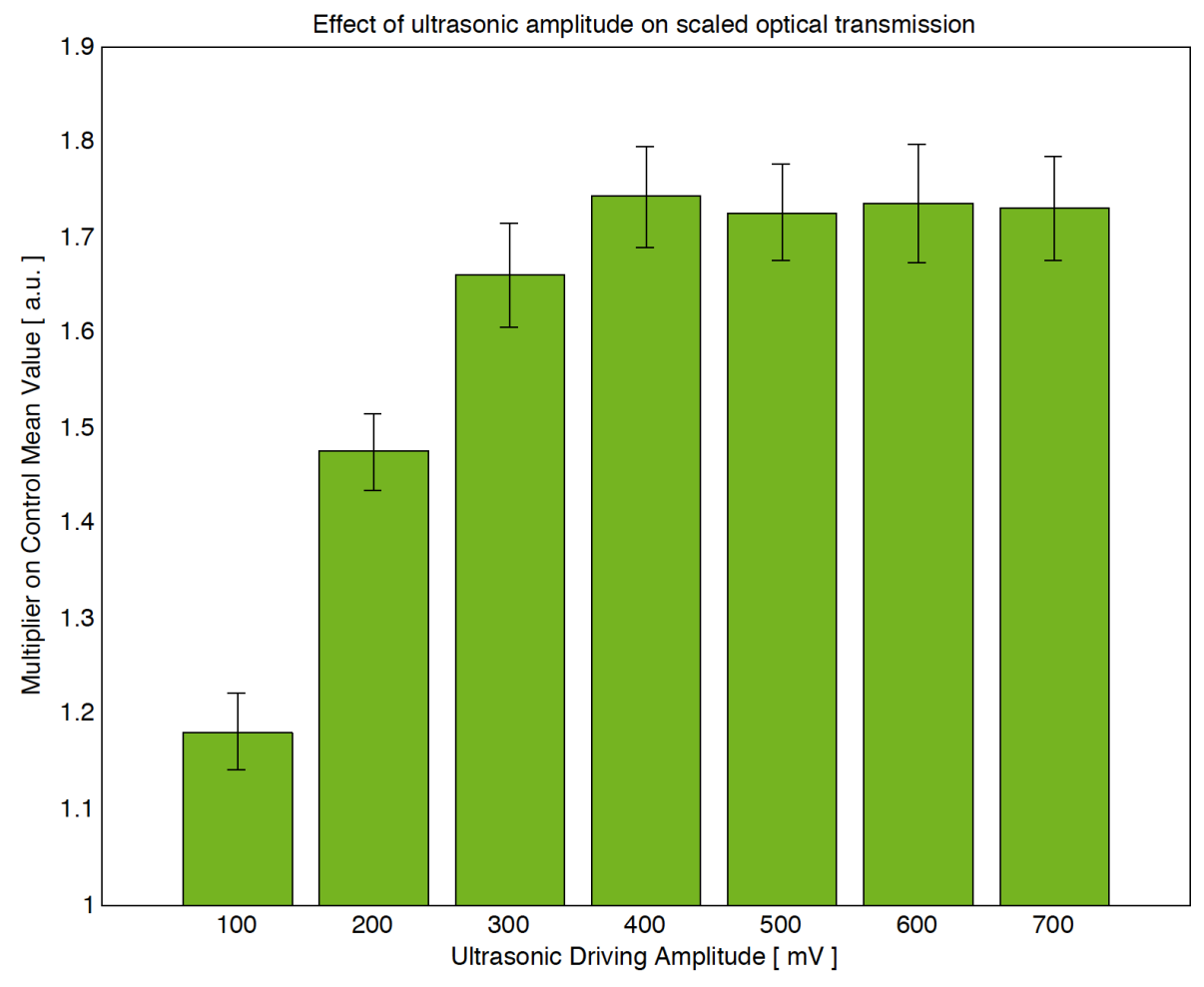

FIGURE 4.12 When scaled against the control mean value, increasing ultrasonic driving amplitude improves the transmitted transdermal optical power by a maximum factor of 1.742 times the control mean.

variation in laser intensity from between pulses coincided with significant deviations in the photocurrent at corresponding indices. To account for this source of error, the normalized laser energy was therefore scaled using the standard deviation of the photocurrent, which was then subtracted from the photocurrent data.

The resultant data was translated into units of optical power based on the logarithmic regression coefficients included in Table 4.3. The optical power data is depicted in Figure 4.11, grouped by ultrasonic driving voltage. After observing the significant change in transmitted optical power, the data was scaled by the mean of the control sample to demonstrate the factor by which the optical power increased. Figure 4.12 depicts the mean improvement factor observed under each driving amplitude 
and demonstrates that low intensity ultrasound consistently increases optical power with increasing ultrasonic amplitude. The maximum mean improvement factor was recorded as $1.742 \pm 0.0526$ times the control mean under $400 \mathrm{mV}$ driving voltage, which plateaued to an average $1.733 \pm 0.0549$ times the control mean. The error bars shown, which represent one standard deviation above and below the respective mean, also confirm that improvement factors recorded at driving amplitudes beyond $400 \mathrm{mV}$ did not statistically differ from the maximum observed value. The full process of error analysis was performed in MATLAB and is made available in Appendix C.

\subsection{Discussion}

This project hypothesized that the paraxial application of continuously pulsed ultrasound and laser illumination would substantially modulate the optical properties of the local tissue such that an increase in total transmitted intensity might be observed. In that regard, a calibration correlation was established to relate the measurable photocurrent generated by light transmitted into a sealed integrating sphere with the incident optical power upon the measurement device. Although theory had predicted in an ideal setting that the relationship would be strictly linear, the data shown in Figure 4.10 suggests that the improvised measurement system employed in these experiments was only approximately linear for a short region, but became increasingly less accurate beyond that region. Comparing the least-squares coefficients of the linear and logarithmic regressions, included in Table 4.3, revealed that the $\log$ fit exhibited a smaller Mean Squared Error and a slightly higher coefficient of determination, which confirmed that measuring the voltage drop across a series 

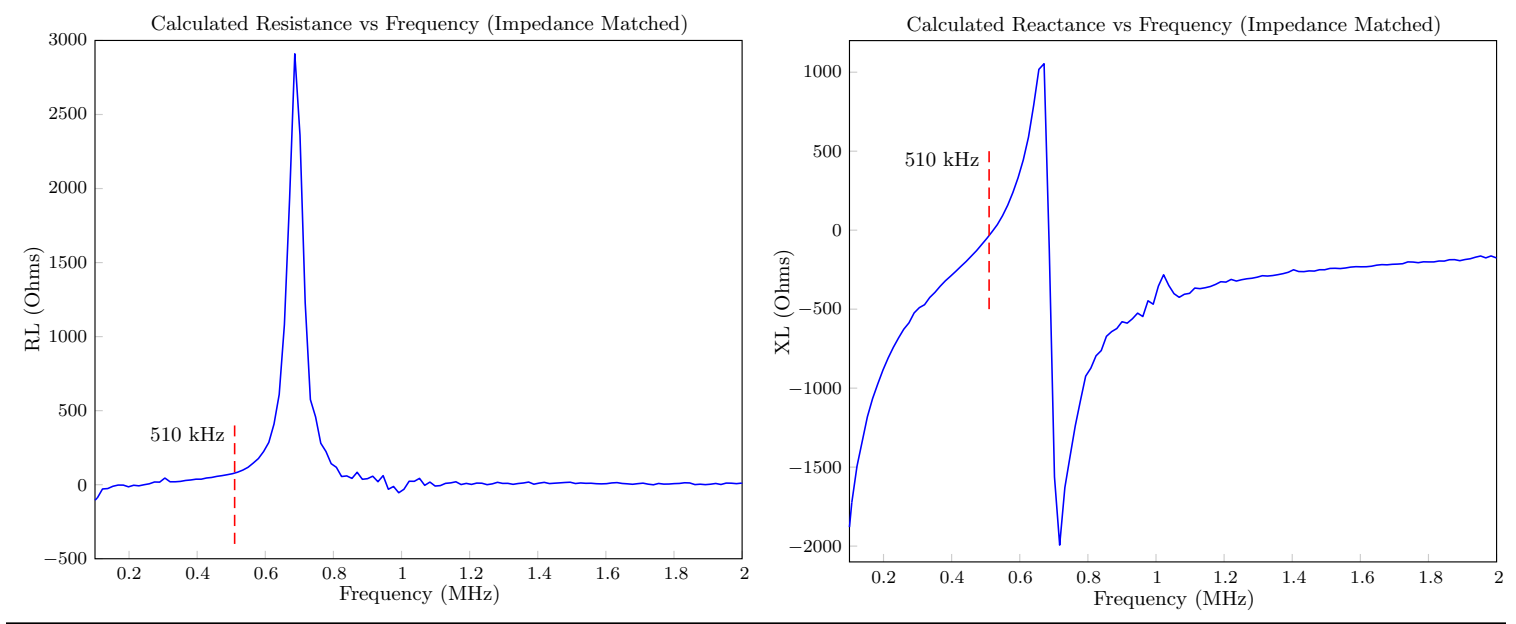

FIGURE 4.13 After incorporating an L-match impedance matching circuit, the resistance of the $500 \mathrm{kHz}$ transducer dropped to $74.21 \Omega$ while the reactance dropped to $-51.65 \Omega$.

resistor did not permit the linear translation of photocurrent to optical power. The method of measurement represented an indirect calculation of photocurrent, and so necessitated the assumption that the imposition of the voltage probe upon the system would negligibly affect the current measurement. However, should this assumption not hold true, the system would likely behave somewhere between the original logarithmic relationship and the ideal linear correlation, as was observed. Nevertheless, recording the approximate photocurrent in this manner did result in a logarithmic relationship that was less concave than measuring the photovoltage directly, which was preferable for the purposes of these experiments.

With regard to transmitting the ultrasonic driving signals to the transducers, electrical impedance matching circuits were designed and calculated by the data included in table 4.2. After incorporating the impedance matching circuit, the effective resistance of the circuit + transducer decreased from $89.01 \Omega$ to $74.21 \Omega$ while the reactance decreased from $-630.73 \Omega$ to $-51.65 \Omega$, as shown in Figure 4.13. These changes 
resulted in a total impedance magnitude of $|Z|=90.42 \Omega$, which although not ideal was considerably closer to the goal $50 \Omega$ impedance than the original impedance of the transducer. The impedance magnitude spectra for both the $500 \mathrm{kHz}$ transducer on its own and the impedance matched transducer pair are included in Figure 4.14. The plots demonstrate the drastic difference in impedance "seen" by the sine wave transmitted from the RF amplifier toward the transducer and the importance of appropriate impedance matching in electrical circuits.

However, the most appropriate method of realizing the impact of the impedance matching circuit on the power transmission is using the source-load reflection coefficient, $\Gamma_{S L}$, which can be calculated using equation 4.16 below, and provides a measure of the portion of the the signal that will be reflected from the interface between two circuits of differing impedance. The original reflection coefficient of the transducer was $85.44 \%$ of the incident energy, which resulted in minimal acoustic generation and significant losses in the system. The impedance-matched system, however, operated with a reflection coefficient of $28.79 \%$, which permitted significantly more power transmission and acoustic generation.

$$
\Gamma_{S L}=\frac{Z_{L}-Z_{S}}{Z_{L}+Z_{S}}
$$

The RF amplifier incorporated two digital displays that provided a measure of the power forward and power reflected from the circuit + transducer pair. This data was recorded, however, the displays did not provide sufficient significant figures to be of practical use in measurement. That being said, the average reflected power 

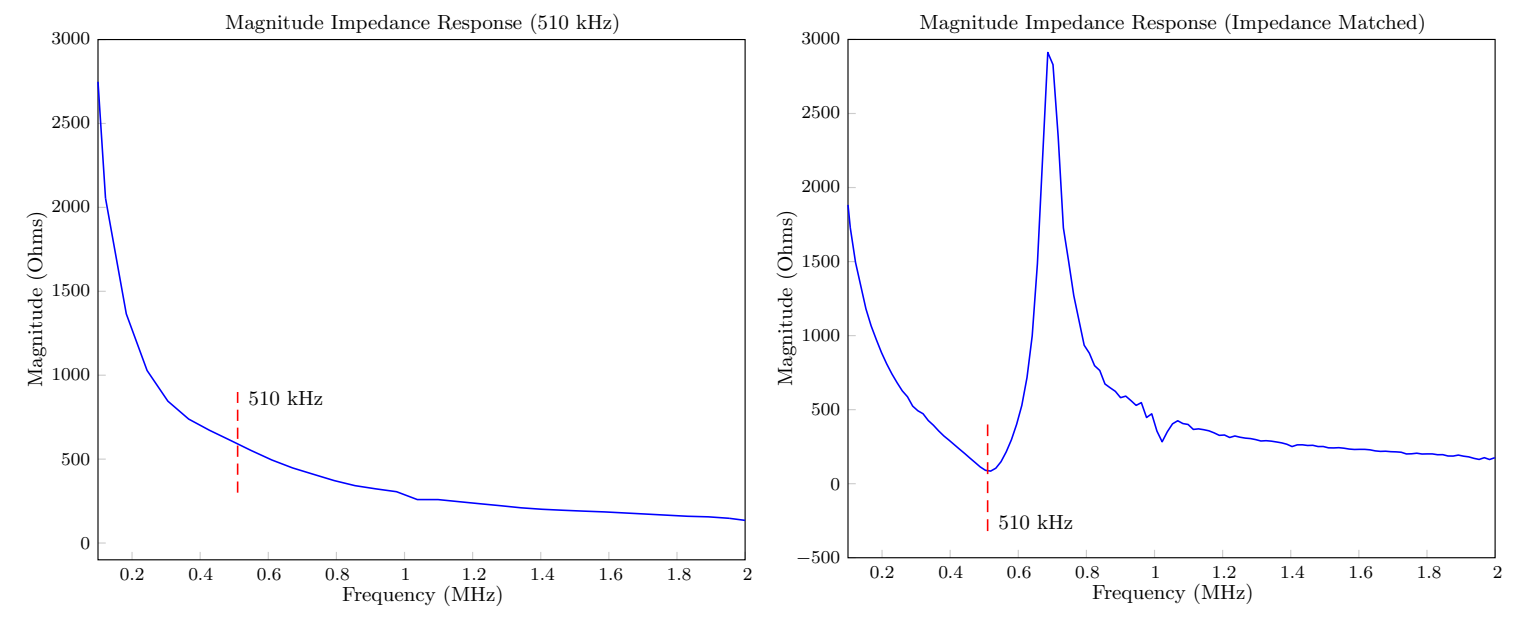

FiguRE 4.14 [Left] Plot of the impedance magnitude of the $500 \mathrm{kHz}$ transducer. [Right] Plot of the impedance magnitude of the impedance-matched transducer. The matching circuit was designed to drag down the impedance of the transducer at the central frequency of $510 \mathrm{kHz}$.

coefficient throughout the testing amounted to approximately $16.69 \%$, which suggests that the physical impedance matching circuit functioned better than the analysis would have predicted based on the source-load reflectance coefficient calculated above. However, it is worth noting that the method of impedance analysis only provided an estimate, which is not to be equivalated to the analysis that could be provided by a specialized RF impedance measurement device. This discrepancy may account for the difference in analyzed performance as compared to the qualitative observations regarding reflected power. Nevertheless, the impedance matching circuit allowed for ample power transmission to generate acoustic pulses, to the point that under high amplitude pulsation, the transducer was able to incite the generation of a column of bubbles in water.

After calibrating the relationship between incident optical power and measured photocurrent and more closely matching the effective electrical impedance, the ul- 
trasound modulation experiments revealed that increasing ultrasonic amplitude corresponded with a consistent improvement in transmitted optical intensity through $4 \mathrm{~mm}$ of porcine skin, consisting of epidermis, dermis, and adipose tissue. The data in Figure 4.11 distinctly shows isolated increases in transmitted intensity as compared to the control sample with no ultrasound applied. This data accounted for known sources of error resultant from variations in laser energy between pulses, intermittent high frequency interference from nearby electronics, and a wandering baseline related to the exposed leads of the series resistor. In that regard, normalizing the laser energy data determined the unitless relative deviance due to variations in pulse output energy corresponding directly with peak photocurrent measurements of the same point index. This data was scaled and deducted using the respective standard deviation of the photocurrent to countermand the effect that fluctuations in output laser intensity imparted upon the measured change in transmission. Additionally, the margins of error were further reduced by the omission of outliers, which occurred regularly every 12 th data point as the result of a high frequency pulse interfering with the signal. It is suspected that this pulse was associated with the cycling of the pulsed power circuit driving the laser. Finally, observation of the difference in normalized datasets revealed that the baseline intensity of the measurement probe was not consistent throughout the tests. This phenomenon, commonly referred to as a "wandering baseline" in the analysis of data from electrocardiograms (ECG's), was accounted for by means of deducting an 11-point moving average from the photocurrent, which stabilized the baseline while preserving the random measurement error associated with the photodiode and variations in optical scattering and anisotropy. 


\begin{tabular}{lll}
\hline \hline Pulsation Amplitude & Mean Amplification Factor & Standard Deviation \\
\hline $0 \mathrm{mV}$ \{control $\}$ & 1.0 & 0.0306 \\
$100 \mathrm{mV}$ & 1.1813 & 0.0398 \\
$200 \mathrm{mV}$ & 1.4755 & 0.0400 \\
$300 \mathrm{mV}$ & 1.6607 & 0.0549 \\
$400 \mathrm{mV}$ & 1.7421 & 0.0526 \\
$500 \mathrm{mV}$ & 1.7261 & 0.0503 \\
$600 \mathrm{mV}$ & 1.7373 & 0.0613 \\
$700 \mathrm{mV}$ & 1.7298 & 0.0554 \\
\hline \hline
\end{tabular}

TABLE 4.4 Amplification factor statistics by ultrasonic driving amplitude

The resultant intensity data varies randomly throughout each individual test group. This may also suggest that the duration of application was not an important factor in amplifying the transmission, but was certainly indicative of its irrelevance for durations less than 210 seconds. Moreover, the distinct jumps between test groups are further indicative of an immediate response of the tissue to the application of the ultrasound, as a delayed or prolonged response would have been observed in the wandering baseline. This assertion could be confirmed in future experimentation by comparing the application of ultrasound in order of both increasing and decreasing intensities. Additionally, Figure 4.12 demonstrates that the increase in transmitted intensity appears to approach an upper threshold. The amplified mean values are included in Table 4.4 and reach a plateau around an average amplification factor of $1.733 \pm 0.0549$ times the control mean. Prior research into the mechanisms behind UOT suggest that the changes in mass density and optical phase due to the periodic compressions and rarefactions of tissue reduce scattering and permit a deeper penetration depth into the tissue. However, it is reasonable to conclude that the tissue may only be modulated so much before the application of ultrasound ceases to further 
alter tissue properties. Consequently, it is speculated that increasing the ultrasonic amplitude beyond the observed values would likely not result in a measured deviation from this plateau, until such point as the application resulted in a significant transformation of the local tissue structure. For example, the application of high intensity ultrasound may result in the cavitation of the local tissue by means of sonophoresis, which may further alter the optical properties beyond the observed upper threshold. Alternatively, operating at different frequencies may also vary the maximum amplification factor, although a similar curve and plateau would be expected.

\subsection{Conclusions and continued research}

This series of experiments successfully confirmed the potential for ultrasonic pulsation to be utilized to mechanically alter the optical properties of ex vivo tissue, encouraging increased transdermal light transmission through porcine analogues of human skin. With regard to potential applications, the increase also implies a reduction in light either back-scattered or absorbed within the tissue, which would permit a greater proportion of incident energy to be delivered to a clinical target below the epidermis. The combination of two well-established clinical technologies in this manner boasts the potential to both improve procedural efficacy and to reduce the severity of detrimental side effects resultant from unwanted absorption by biological chromophores, such as epidermal melanin. Moreover, the potential to effectively bypass undesirable melanin absorption may allow for the expansion of light-based procedures to patients of darker complexions.

Founding this mechanical tissue optical clearing technique on low intensity ultra- 
sound also offers the option to further improve the technique by either incorporating chemical OCAs to further clear the tissue or otherwise increasing the ultrasound intensity to induce cavitation within the tissue. Continued research into this technology should investigate a spectrum of ultrasonic amplitudes and frequencies to more thoroughly establish tissue behavior throughout and beyond the intensities investigated herein. Additionally, the project will investigate other design applications in order to implement paraxial pulsation with traditional free space optical transmission devices used in contemporary clinics while also investigating behavior under clinically relevant laser energy levels. For example, refracting the ultrasound into the tissue using a silicone elastomer wedge may permit application with free-space beams, but also may operate at a sufficiently high angle of incidence to be beyond the aforementioned threshold between GRIF operations. With regard to project specifics, future efforts would do well to investigate:

- a continuous range of increasing ultrasonic amplitude to include amplitudes sufficient to generate microcavities within the tissue, thereby effecting a physiological change and potentially further improving forward transmission;

- differing incident angles for both the laser and acoustic propagation vectors to characterize the regimes of GRIF operation;

- a variety of acoustic frequencies, particularly around the primary and secondary thresholds tabulated in Table 4.1 by choosing a handful of different ultrasonic transducers and generating acoustic waves throughout their central $6 \mathrm{~dB}$ bandwidths, to characterize the efficacy of sonoillumination by pulse frequency. 


\section{Chapter 5}

\section{Backward-mode waveguide-mediated photoacoustic tomography}

Portions of this chapter have been reproduced with permission from the manuscripts entitled Planar waveguide light transmission modality for backward-mode photoacoustic tomography, authored by Mason W. Schellenberg, Paul J.D. Whiteside, and Heather K. Hunt, along with the Master's Thesis entitled Photonic Ablation via Quantum Tunneling authored by Paul J.D. Whiteside, Bioengineering Department, University of Missouri, May (2015) [66, 67].

\subsection{Introduction}

The field of photoacoustic tomography represents a significant divergence from traditional acoustic technologies involving pulsed ultrasound, in that the acoustic pressure waves are produced from within the medium, rather than applied to its surface. Specifically, photoacoustics refers to the phenomenon wherein a short burst of optical radiation generates a subsequent ultrasonic pulse through the rapid thermoelastic expansion of an optical chromophore, such as a blood vessel or melanotic tumor [174]. As such, photoacoustics has been utilized in a wide variety of applications 
ranging from the detection of circulating tumor cells and blood oxygenation to the evaluation and optical characterization of surfaces and thin films [62, 136, 144, 196198].

In typical photoacoustic applications, a laser beam of sufficiently short pulse duration is absorbed by an optical chromophore, causing the absorber to undergo a rapid thermoelastic expansion, which generates an ultrasonic pressure wave that propagates throughout the medium. Much like traditional ultrasound, these acoustic waves carry information about the underlying tissue structure and may be recorded and analyzed to determine the depth of a single acoustic source or to generate a 2D or 3D tomographical map of the tissue structure [60, 174]. However, unlike traditional ultrasound, the acoustic information is based on the optical properties of the medium rather than acoustic differences between tissues [159]. This distinction permits the imaging of acoustically similar tissues based on differences in relative optical properties. For example, Zhang et al. have used photoacoustics to reconstruct 3D tomographical maps of the in vivo vasculature of mouse models, relying on the strong optical absorption of hemoglobin as compared to the surrounding dermal tissues [199]. Such structures are typically unresolvable by traditional ultrasonics, as those techniques predominantly rely on differing material properties, such as compressibility, density, and acoustic impedance, to resolve the boundaries of interfacing materials, such as bone and soft tissue [200, 201].

However, despite providing useful and relevant information regarding previously unresolvable structures, photoacoustic tomography has seen limited clinical implementation owing to the necessity for the technique to be performed in "backward 
mode." This backward mode of operation refers to the parameter wherein the ultrasonic pressure waves must be detected on the optically irradiated side of the tissue, due to the potential interference of echogenic structures, such as bone or lung tissue, and the reduction in signal to noise ratio (SNR) associated with acoustic attenuation through acoustically anisotropic tissues [63].

Typical ultrasonic detectors utilized in both ultrasonic tomography and laboratory photoacoustics are based on piezoelectric elements, which are cheap to manufacture and can be constructed in a wide range of configurations from large low frequency individual elements to high frequency linear or matrix arrays. However, problems in backward mode photoacoustics arise, because piezoelectric materials are typically opaque and therefore complicate the laser irradiation component of the backward mode design by occluding the path of the light when applied coaxially. This has led to the development of three prevailing solutions: (1) to design acoustic detectors that incorporate through-holes to permit coaxial optical fiber radiation of tissue, (2) to spatially separate the acoustic detector from the tissue using a transparent acoustic couplant to transmit light at an angle to the tissue surface, or (3) to use complex dual-wavelength optical detection systems to irradiate tissue through dichroic coatings [63, 159, 202].

Whereas each of these methods has shown promise, each also incorporates additional challenges. For example, when incorporating a cavity through which an optical fiber may irradiate the tissue, the through-hole necessitates changes to the surface detection geometry of the transducer. This alteration from a "disk" to a "ring" geometry introduces an additional edge near the center of the transducer, which can 
subsequently introduce acoustic ringing in the detected signal. The ringing effect may also introduce acoustic artifacts and overwhelm otherwise valuable information, causing a decrease in SNR and a loss of resolution; accordingly, this solution has not seen much development outside of some initial investigations by the Viator laboratory. With regard to the second solution, however, the spatial distancing of the detector from the tissue surface increases acoustic attenuation and signal loss, while also allowing acoustic signals to reflect within the detection chamber, both of which can also decrease SNR. The third solution does not experience such problems, as the opaque transducer is replaced with a dichroic-based optical detector. Such systems use two lasers simultaneously, one to generate photoacoustic pulses by transmitting through the dichroic cavity, and the other to scan and detect changes to the geometry of the cavity resultant from acoustic pressure variations [60,63]. These systems can demonstrate remarkable resolution, as the detection surface is strongly correlated with the spot size of the interrogating beam and the translational resolution of the scanning stage or deformable mirror. However, such systems require two separate lasers along with complex optical components that necessitate rigid, reliable alignment of both incident beams. Although these systems produce exceptionally accurate reconstructions and can be used for in vivo analysis in backward mode, due to their complexity they are often ill-suited for portable clinical applications.

\subsection{Technical discussion}

This project represents an alternative solution to the backward mode paradigm, using a planar selective release waveguide to irradiate the tissue directly underneath 
the detection surface of an ultrasonic transducer or transducer array. The principal benefit of this modality lies in its ability to utilize readily available acoustic detection equipment and software that are calibrated for use with piezoelectric elements, which greatly simplifies any potential commercial and clinical applications of the technology as compared to more complex approaches. The following sections describe the fundamental science behind photoacoustic signal generation and our proposed method of photoacoustic tomography.

\subsubsection{Photoacoustics}

As discussed above, photoacoustics is the result of the rapid irradiation of an optically absorbing particle by a short pulse of light. This photo-mechanical effect may accompany the thermal expansion of any chromophore following a pulse of optical irradiation; however, the resultant acoustic pressure wave is only useful for photoacoustic tomography in the event that two conditions are met. The first condition, known as "stress confinement," stipulates that the pulse duration of the incident laser light must be shorter than the time required for the resultant acoustic wave to depart. The condition for stress confinement is shown in Equation 5.1 below:

$$
\tau \leq \frac{\delta}{c_{s}}
$$

wherein $\tau$ is the laser pulse duration, $c_{s}$ is the speed of sound in the medium, and $\delta=1 / \mu_{a}$, where $\mu_{a}$ is the optical absorption coefficient of the target [144]. The second condition, known as "thermal confinement," necessitates that the energy absorbed by 
the chromophore must not be dissipated in the form of heat before the acoustic wave can depart. When firing a laser with a pulse width on the order of a few nanoseconds, the heat dissipation away from the absorber may be neglected and all of the incident electromagnetic energy is translated into acoustic pressure [203]. This pressure wave can be described mathematically by Equation 5.2 below:

$$
p(x, t)=\frac{1}{2} \Gamma_{g} \mu_{a} H_{B} e^{-\mu_{a}\left(x-c_{s} t\right)}+\frac{1}{2} \Gamma_{g} \mu_{a} H_{B} e^{-\mu_{a}\left(x+c_{s} t\right)}
$$

wherein $\Gamma_{g}$ represents the Grünesian coefficient, $H_{B}$ is the radiant exposure of the beam, $x$ is depth, and $t$ is time $[204,205]$. At the surface of an ultrasonic detector, where $x=0$, the equation describes the recorded amplitude of the pressure wave as a function of time. As the wave approaches the detector surface, the pressure exponentially increases in amplitude followed by an immediate drop when the wave hits the detector. Then, while the inverted negative pressure wave is reflecting away from the detector, the recorded pressure follows a reciprocal exponential return to the baseline. Peak-to-peak analysis is often used in photoacoustic experimentation, as a result of this characteristic waveform profile observed by the detector.

\subsubsection{Backward-mode transmission modality}

Following the principles of photoacoustic signal generation, the majority of photoacoustic systems use pulsed lasers operating with pulse widths on the order of a few nanoseconds or less. A typical laser system for photoacoustic tomography is the Q-Switched Nd:YAG laser, which generates a primary beam at $1064 \mathrm{~nm}$, but whose 


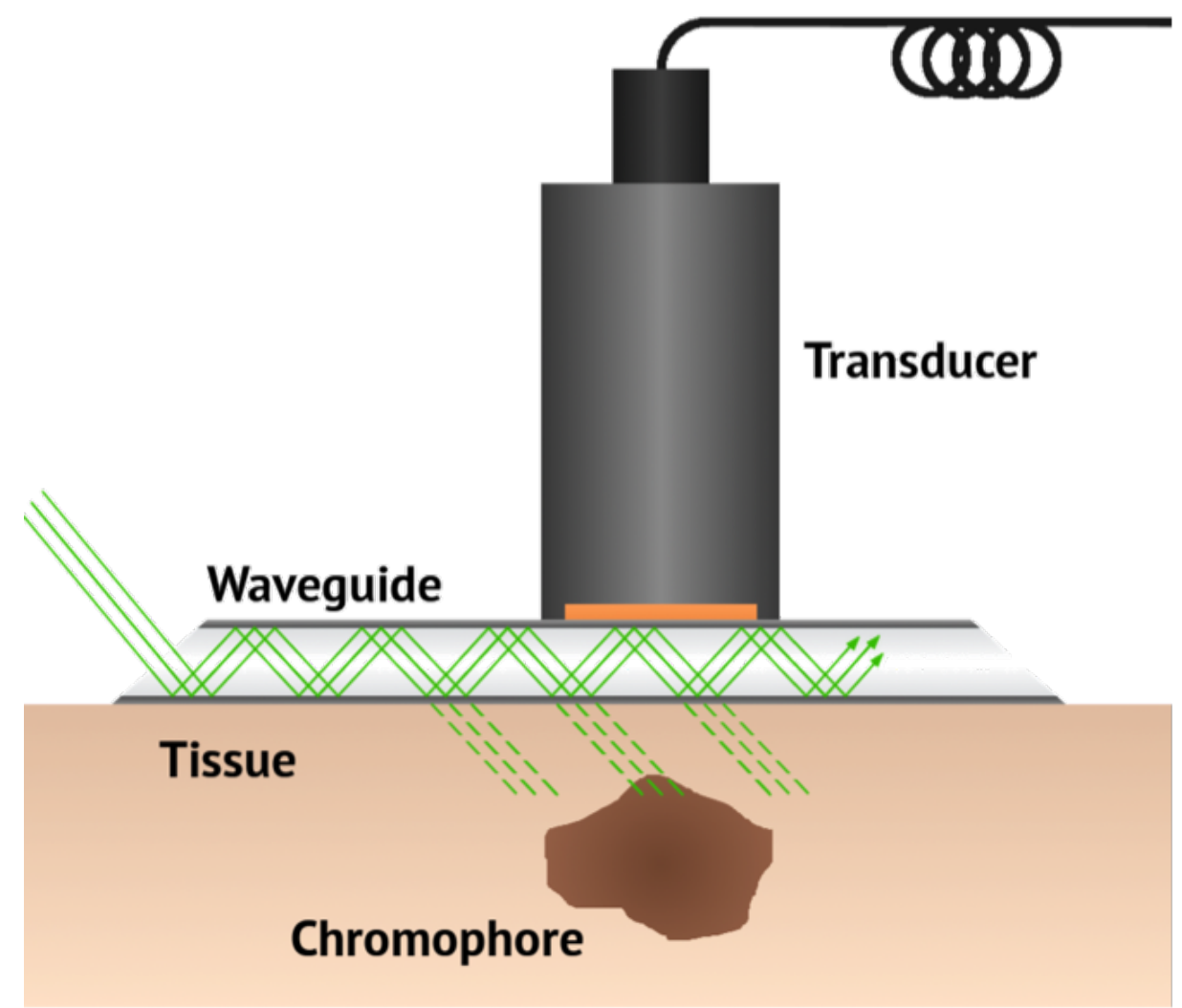

FIGURE 5.1 Transmitting the light through an off-axis waveguide in contact with the tissue allows the incorporation of a standard off-the-shelf transducer on the rear face to detect ultrasonic photoacoustic signals.

secondary harmonic is $532 \mathrm{~nm}$, which is strongly absorbed by biological chromophores such as hemoglobin and melanin. Moreover, some systems utilize the third harmonic at $355 \mathrm{~nm}$ to drive an optical parametric oscillator (OPO), which allows the laser to output pulses at wavelengths throughout the visible spectrum.

The system designed herein transmits the secondary harmonic of a Q-Switched Nd:YAG laser into the tissue using a planar selective release waveguide (SRW) in a manner similar to that discussed in the previous chapter. As the light transmits along the SRW, it is contained within the silver cladding layer until it reaches the designated active area, as shown in Figure 5.1. The light then transmits out through 
the active area into the target medium, which for our purposes is skin tissue, and is absorbed by an optical chromophore. As mentioned above, the short pulse width of the laser generates an acoustic pressure wave whose properties are dependent upon the optical characteristics of the chromophore. This pressure wave propagates up through the tissue, across the SRW, and is detected by the transducer on the rear face of the waveguide.

Unlike some competing systems, this design makes use of a standard piezoelectric transducer element acoustically coupled to the rear face of the SRW. This orientation necessitates that the optical and acoustic characteristics of the SRW be balanced to allow for transmission of the light into the tissue while also permitting the acoustic energy to transmit across the waveguide substrate. For the purposes of these experiments, our waveguides were made from a single sheet of poly-methyl methacrylate (PMMA), which is largely transmissive to visible light and whose acoustic impedance $\left(Z_{P M M A}=3.26 \mathrm{MRayl}\right)$ does not substantially differ from that of tissue $\left(Z_{\text {skin }}=\right.$ 1.99 MRayl). The SRW substrates used in Chapter 3 were composed of fused silica $\left(Z_{F S}=13.1 \mathrm{MRayl}\right)$, which prevented sufficient transmission of acoustic energy due to the significant acoustic impedance mismatch. The acoustic reflection coefficient at the interface between any two media of differing impedances can be represented as:

$$
R=\left(\frac{Z_{2}-Z_{1}}{Z_{2}+Z_{1}}\right)^{2}
$$

wherein $Z_{1}$ and $Z_{2}$ represent the incident medium and the external medium respectfully. That being the case, the reflection coefficient for an FS-tissue interface would be 
approximately 0.542 , whereas for a PMMA-tissue interface, the reflection coefficient would be approximately 0.0585 , which is an order of magnitude more transmissive. We had briefly considered using an acoustic impedance matching layer with the fused silica substrates, however, the available polymers that would have achieved greater acoustic transmission would also have inhibited optical transmission into the tissue by promoting total internal reflection instead. Future designs of the waveguide that operate as planar beam deflectors, which will be discussed later on, may incorporate acoustic matching layers, as they do not operate under the same internal reflection conditions.

\subsubsection{Technical objectives}

Having previously established that collimated light could successfully be transmitted into phantoms simulating human skin through direct contact, this project represented a significant potential application that incorporated an auxiliary sensing technology alongside laser irradiation. This sought to formally establish the potential for using planar waveguide irradiation as a means of achieving backward-mode photoacoustic detection. As such, the primary milestone was pragmatic in that experiments were designed to detect and analyze a simple one dimensional photoacoustic signal measured with a $10 \mathrm{MHz}$ transducer to correspond peak locations with known chromophores. Following that, the technology was expanded to detect and analyze a two-dimensional array of time-resolved photoacoustic traces to reconstruct a map of photoacoustic sources. Accordingly, the goals of this project were as follows:

- Record photoacoustic signals in backward mode using a transducer on the rear 
face of a waveguide and correlate with known chromophore depths;

- Design and fabricate a stand-alone signal multiplexer, incorporating printed circuit boards to multiplex 32 input signals onto 4 oscilloscope channels;

- Record time-resolved photoacoustic data from 32 transducer elements in rapid succession by programming an automated LabVIEW VI;

- Reconstruct a 2D depth profile of a tissue phantom or ex vivo tissue sample in MATLAB using the $\mathrm{k}$-space reconstruction methods.

\subsection{Materials and methods}

The following sections describe the procedures involved in waveguide fabrication and tissue sample preparation, along with a description of the optical apparatus employed and the electronic instrumentation components and procedures. The experiments discussed herein represent two distinct phases of development: (1) empirically establishing the technique by recording preliminary photoacoustic data in a backward mode, and (2) revising the apparatus to record data in a linear array and thereby generate a two-dimensional profile.

\subsubsection{Porcine tissue preparation}

The porcine tissue samples for the preliminary research were prepared according to the same protocols designed for aforementioned sonoillumination experiments. Included for ease of reference, large sections of ex vivo porcine skin were donated for this research project from the Mizzou Meat Market (College of Agriculture, Food, and Natural Resources at the University of Missouri). The skin was disinfected in a 1.75\% ethanol solution for 2 minutes prior to processing for experimentation. Following disinfection, the sections were trimmed down to a thickness of $4 \mathrm{~mm}$, incorporating epidermal, dermal, and adipose tissue, and cut into $40 \mathrm{~mm}$ square samples. Originally, each sample was held taut and adhered at the edges with superglue over a thin fiberglass mesh to individual 3D-printed tissue mounts, as shown in Figure 4.4, 
which incorporated open apertures to allow for measurement of optical transmission through the tissue. The fiberglass mesh was included to provide additional support to the porcine skin and prevent potential indentation of the tissue during testing. Processed skin samples were wrapped in moist Kim wipes to prevent dehydration, enclosed in air-tight plastic bags, and stored in a freezer at $4^{\circ} \mathrm{C}$ until needed. Prior to use, samples were defrosted by running water over the plastic bag for $10-15 \mathrm{~min}$.

\subsubsection{Turbid tissue phantom design}

Due to the complexity and variability of biological tissues, while developing the $2 \mathrm{D}$ photoacoustic imaging modality, optical tissue phantoms were utilized as a controllable substitute for skin. The preparation protocol, adapted from procedures published by Chen et al. , is based on a mixture of intralipid, methanol, agar, and gelatin to generate an optically scattering gel with a speed of sound comparable to that observed in tissue [206]. This procedure was refined through photoacoustic analysis to closely match the speed of sound in test samples with the reported speed of sound in tissue. Samples were produced by heating $13.73 \mathrm{~mL}$ of ultra-pure DI water to $30^{\circ} \mathrm{C}$, mixing the solution using a magnetic stir bar at $350 \mathrm{rpm}$, and then adding $5.472 \mathrm{~mL}$ of $20 \%$ Intralipid, $2.94 \mathrm{~mL}$ of methanol, and $17.6 \mu \mathrm{L}$ Formaldehyde $37 \%$. After the solution is homogenized, the mixing speed was increased to $700 \mathrm{rpm}$ at which point $1.54 \mathrm{~g}$ of gelatin (300 Bloom) and $190 \mathrm{mg}$ of agar were slowly added to the solution, such that the powder did not aggregate in the solution. The beaker was covered with an aluminum heat shield and was slowly heated up to $45^{\circ} \mathrm{C}$. Once reached, the solution was poured into a tissue phantom mold, which was then transferred into a vacuum desiccator for $10 \mathrm{~min}$ to remove dissolved gas from the solution and minimize the occurrence of air pockets. The mold was then placed in a $4^{\circ} \mathrm{C}$ refrigerator for 1 day to allow the solution to set. 

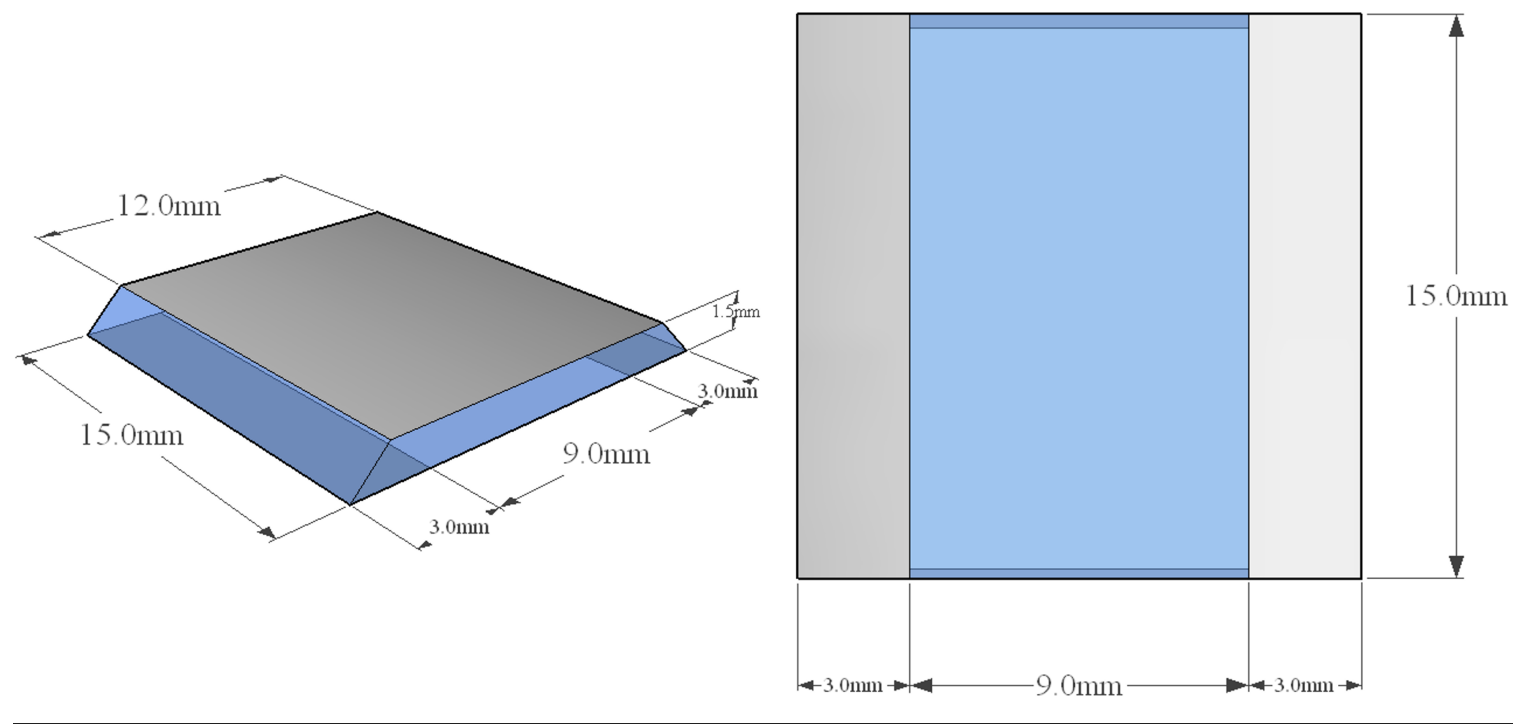

FiguRE 5.2 [Left] The original design for the photoacoustic waveguides utilized PMMA substrates clad in silver and titanium; [Right] the active area of the waveguides exposed the bare substrate, relying on silver to confine the light elsewhere within the waveguide, which was later revealed to provide a source of error owing to photoacoustic signals incited within the metal cladding [67].

\subsubsection{Waveguide fabrication}

The original waveguides for this project were fabricated from $1.5 \mathrm{~mm}$ thick sheets of PMMA (Evonik CRYO, 0A000 GT). This material was chosen for its relatively low acoustic impedance, given that it is commercially available in transparent slabs. There are other materials that would have resulted in a closer acoustic impedance match to skin $\left(Z_{\text {skin }}=1.99 \mathrm{MRayl} ; Z_{\text {acrylic }}=3.26 \mathrm{MRayl}\right)$; however, few of them are transparent at $532 \mathrm{~nm}$ or available in rigid planar sheets [207, 208]. Two different waveguide designs were utilized for these experiments, with the second representing a revision of the first following the results of preliminary testing. The original waveguides were similar to those fabricated in Chapter 3 , in that they were planar slabs coated in titanium and silver with $45^{\circ}$ polished entrance apertures, as depicted in Figure 5.2. The substrates were fabricated in-house to be $15 \mathrm{~mm}$ square units, cutting a bevel at $45^{\circ}$ and polishing the bevel with increasing grit optical sandpaper, finally followed by a fine scratch removing compound specifically formulated for plastics (Novus 
Fine Scratch Remover No.2). The two angled entrance and exit apertures of the substrates were masked off with Kapton tape, along with a $9 \mathrm{~mm}$ x $15 \mathrm{~mm}$ rectangular section of the largest face $3 \mathrm{~mm}$ from each end, which would serve as the waveguide's active area. The substrates were then clad in thin films of titanium and silver using a cold sputtering vacuum deposition system (Quorum Technologies, K575X) to serve as mirrors on the bounding surfaces of the substrate. The titanium was deposited using a $99.998 \%$ pure Ti target at $90 \mathrm{~mA}$ for $60 \mathrm{~s}$, which formed a film less than $10 \mathrm{~nm}$ thick. The titanium acted as an adhesion layer for the silver, which was deposited from a 99.95\% pure Ag target at $30 \mathrm{~mA}$ for 300 s to form a much thicker layer approximately $200 \mathrm{~nm}$ thick. Laser light incident perpendicularly upon one of the angled faces would internally reflect along the waveguide to the exit aperture when exposed to air, but would transmit out through the active area when in contact with tissue.

The results of our preliminary testing, which will be discussed in the sections to follow, revealed that cladding the waveguides in silver incorporated significant noise into the photoacoustic signals owing to a photoacoustic response incited within the metal cladding. Consequently, for the continued experiments, the waveguide was redesigned such that it did not incorporate an optically absorbing cladding. The new waveguides have been revised such that they are comprised of two acrylic slabs fused together using Norland Optical Adhesive 13685 (NOA 13685). This adhesive was chosen based on its reported refractive index of $n=1.3685$, such that light incident at $45^{\circ}$ within the acrylic substrates $(n=1.4937)$ would internally reflect from the interface and be directed perpendicularly into the tissue. In this manner, the waveguide behaves as a planar optical beam deflector, rather than a traditional multiply-reflecting waveguide. However, at the moment, the fabrication protocols for the new waveguide design are still under development and revision, so this section will be revised following the completion of that design. 


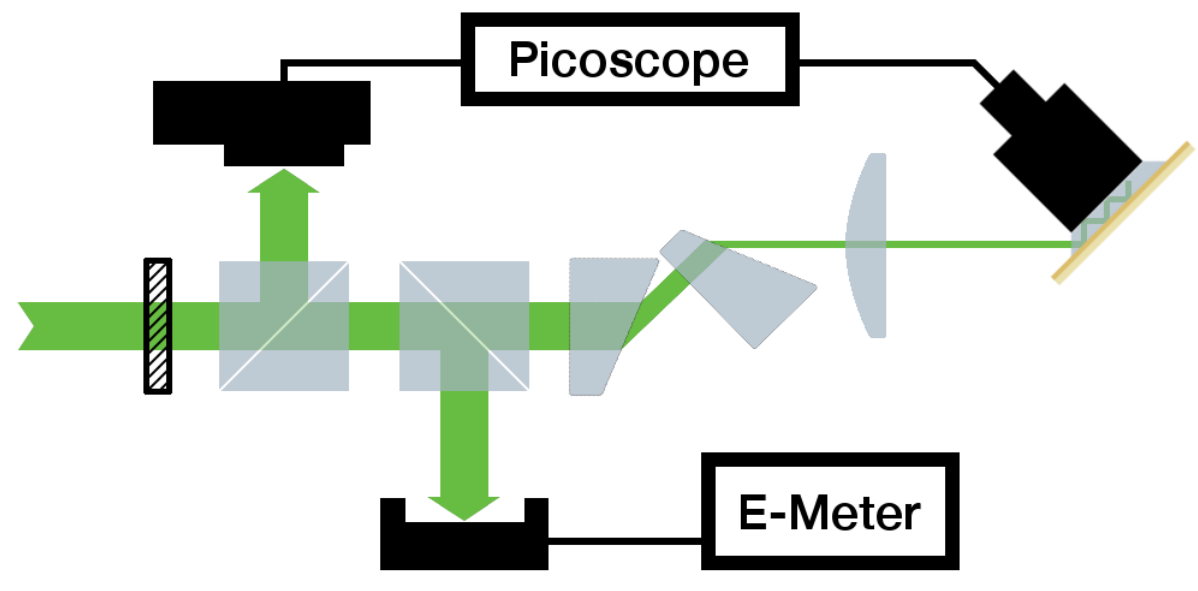

FigURE 5.3 The optical apparatus was similar to previous systems, transmitting light through a beamsplitter to trigger data collection and measure laser pulse energy, compressing the beam along one axis using an anamorphic prism pair, re-collimating the beam, and transmitting light through an SRW with an ultrasonic transducer on the rear face.

\subsubsection{Experimental apparatus}

The experimental apparatus involved a number of custom designed and fabricated materials and circuits, in addition to standard optical components and electronic instrumentation. These materials and systems were revised between the two phases of research to account for revelations and updated operating conditions. Accordingly, the sections that follow describe each of these components in detail.

\subsubsection{Optical apparatus}

The first phase of the project was intended to demonstrate backward-mode photoacoustics with simple one dimensional generation and detection of photoacoustic waveforms in porcine skin. As such, the optical apparatus was markedly similar to those discussed in previous chapters, with some slight discrepancies, as shown in Figure 5.3. The light from a pulsed Nd:YAG laser transmitted first through a zero-order half-waveplate (Thorlabs, WPH05M-532) and polarizing beamsplitter cube (Thorlabs, CM1-PBS251). Rotating the waveplate rotates the incident linear polarization with respect to the cube, resulting in what is known as a "variable beam splitter," in 


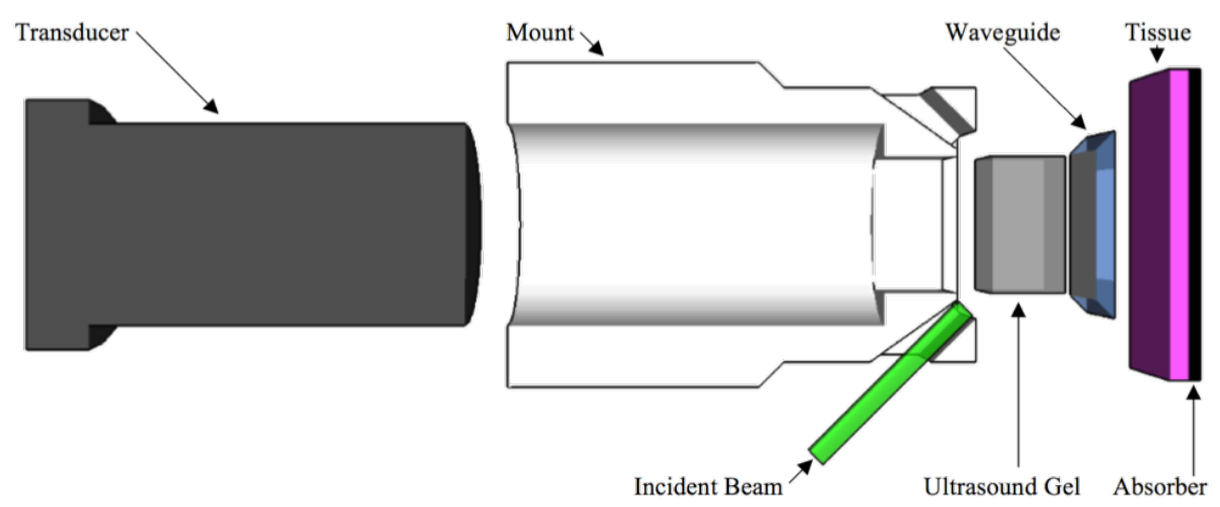

FigURE 5.4 The waveguide and $10 \mathrm{MHz}$ ultrasonic transducer were incorporated into a single removable $3 \mathrm{D}$ printed cartridge that incorporated both components and a cavity filled with ultrasound gel to transmit the photoacoustic signals.

that the rotation angle of the waveplate determines the relative portion of s- and ppolarized light that either deflects toward the energy meter or transmits toward the waveguide.

The light that is deflected is used to trigger data collection by generating a photovoltage pulse using a high-speed photodiode (Thorlabs, DET10A), which was chosen for its exceptionally fast rise time of $1 \mathrm{~ns}$ and sensitivity at $532 \mathrm{~nm}$. The light that transmits toward the waveguide also transmits through a 50:50 beamsplitter cube, which directs half of the intensity toward a pulse power meter (Thorlabs, PM100USB), which measures the pulse energy of the laser to account for variations in intensity between pulses. The other half of the energy transmits through a $4 \mathrm{X}$ anamorphic prism pair (Thorlabs, PS883-A), which double refracts the beam between two fixed-angle prisms and thereby compressed the beam along one axis to transform the beam from a circular Gaussian profile into an elliptical Gaussian approximately $1.5 \mathrm{~mm}$ thick along its narrow axis. The extreme degree of compression resulted in significant dispersive effects, which were corrected using a long focus cylindrical lens (Thorlabs, LJ1558L2-A), which re-collimated the beam prior to entering the waveguide.

The waveguides were fabricated as described in the previous section and were 
fixed at a $45^{\circ}$ incline with respect to the incident beam using a custom 3D printed optomechanical mount, as shown in Figure 5.4. That was rigidly connected to the optical train. Unlike the apparatuses discussed in previous chapters, these experiments did not necessitate an integrating sphere, as we were not measuring transmitted optical intensity. The light transmitted out of the waveguides into the porcine tissue samples, which had ABS plastic absorbers embedded into the dermis to simulate biological chromophores at an appropriate depth. The second phase of this project is currently under development, but is intended to make use of tissue phantoms with tubes filled with an artificial blood analogue to properly characterize the 2D photoacoustic tomography system prior to conducting experiments on ex vivo tissue.

\subsubsection{Electronic apparatus}

The goal of these experiments was to establish a backward-mode method of performing photoacoustic tomography in tissue phantoms and samples with distinct relevance to empirically mapping the severity of thermal injuries. Accordingly, pulsed laser light, generated by a Q-switched Nd:YAG laser (Opotek, Vibrant 355 II) operated at a wavelength of $532 \mathrm{~nm}$ with a $5 \mathrm{~ns}$ pulse width, was transmitted through porcine skin samples. Optical chromophores consisting of a Direct Red dye solution in transparent tubing absorbed the light and generated photoacoustic pulses. These pulses were detected by a custom-ordered 32 channel ultrasonic transducer array, which operated with a $12.5 \mathrm{MHz} \pm 10 \%$ central frequency. The array was designed to utilize a $-6 \mathrm{~dB}$ bandwidth of $60 \%$ extending from roughly $7.5 \mathrm{MHz}$ to $17.5 \mathrm{MHz}$. Elements were $0.15 \mathrm{~mm}$ wide, with an element pitch of $0.20 \mathrm{~mm}$, an inter element spacing of $0.05 \mathrm{~mm}$, and an element width of $6 \mathrm{~mm}$. The total active dimensions for the 32 consecutive rectangular elements was $6.35 \mathrm{~mm}$ x $6 \mathrm{~mm}$. The array was acoustically impedance matched to PMMA (roughly 3.3 MRayl) to minimize reflections at the interface with the waveguide. Finally, the array operated under a stated $50 \Omega$ output 
impedance; however, the specifications documentation provided by the manufacturer states "Imasonic designs its phased array probes to get real impedance as close as possible to $50 \Omega \ldots$ the real impedance is higher than $100 \Omega$. The capacitive imaginary part is typically not matched."

As described above, the transducer array was connected to a custom-designed 32 channel multiplexer, described in the next section, which amplified four output signals simultaneously, while shunting the unused signals to ground. The output signals of the multiplexer were indexed by an Arduino Mega R3, which received indexing commands via LabVIEW serial output through a USB cable. For any one full 32-element image to be generated, a minimum of 8 laser pulses were required. The output channels of the multiplexer were fed via BNC cable to the four input channels of the Picoscope 5442B USB oscilloscope, incorporating $50 \Omega$ feed-through terminators to minimize reflections in the lines due to electrical impedance mismatching. Laser pulses, controlled by LabVIEW via RS-232 commands, triggered data collection by irradiating a photodiode (Thorlabs, DET 10A).

A LabVIEW automation software was designed to control laser operations, multiplexer indexing, and data acquisition. The software was programmed to index the multiplexer outputs onto their first input channel, fire a single pulse of the laser, trigger data collection, record the laser pulse energy, and save the waveform to a file. Following that procedure, the next multiplexer input channel was indexed and the process was repeated. Image processing was performed in MATLAB using the k-wave toolbox (Available from www.k-wave.org, maintained by Bradley Treeby, Ben Cox, and Jiri Jaros) [209, 210].

\subsubsection{Multiplexer design}

In order to obtain higher quality imaging resolution in the second phase of the project, a 32 channel linear array transducer was custom manufactured by Imasonic (Imasonic 


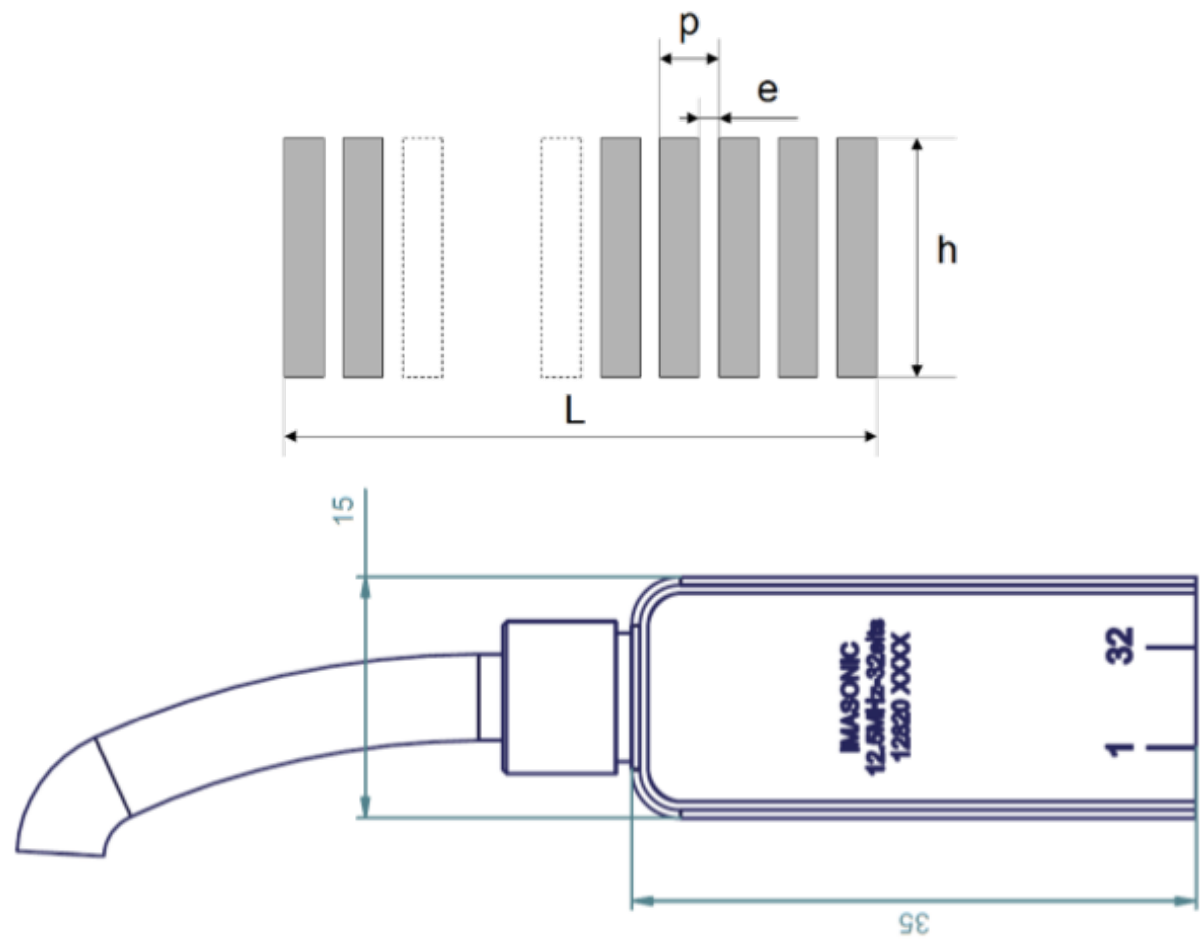

FIGURE 5.5 [Top] The geometry of the array transducer is such that there are 32 elements with rectangular detection areas $\mathrm{h} x(\mathrm{p}-\mathrm{e})$; [Bottom] the array transducer is incorporated into a stainless steel housing with a footprint comparable to the rear face of the standard SRW used in these studies.

SAS, Voray-sur-l'Ognon, France). The linear array was manufactured with a central frequency of 12.5 MHz. Each of the 32 contact areas were designed with an element width of $h=6 \mathrm{~mm}$, an element pitch of $p=0.20 \mathrm{~mm}$, and an element spacing of $e=0.05 \mathrm{~mm}$ for a total active area length of $l=6.35 \mathrm{~mm}$, as shown in Figure 5.5. The array was also acoustically impedance matched to PMMA (3.3 MRayl).

However, given the necessity that each of the 32 channels be recorded individually to preserve signal integrity between channels, and given that the USB oscilloscope (Picoscope 5442B) being utilized was only equipped with 4 channel inputs, a 32 channel multiplexer was required to achieve optimal photoacoustic tomography. Investigations into commercially available multiplexers capable of accepting signals within the appropriate frequency bandwidth exclusively yielded options beyond the limitations of the available grant budget. A thorough literature search revealed that Gamelin 
et al. had developed such a multiplexer for photoacoustic tomography in-house using commercially available electronics components; whereas Gamelin did not provide the circuit diagrams for their proprietary multiplexer, the manuscript listed the part numbers of each of the primary components, including the appropriate operational amplifier chips (op-amps) and the 8-channel multiplexer (mux) chips [211].

Using Gamelin et al. as a foundation, it was determined that the 32 channels would be best multiplexed onto the $4 \mathrm{BNC}$ input channels of the oscilloscope using four individual 8-channel multiplexer boards, which would accept 8 input signals, amplify and output the desired signal to a $50 \Omega$ impedance BNC cable, and shunt the other 7 to ground to reduce cross-talk between signals. However, it was further determined that constructing each of these multiplexer boards using standard through-hole breadboards and wiring would invite excessive external interference and exacerbate signal decomposition. Accordingly, an 8-channel multiplexer circuit was designed as a printed circuit board (PCB) using AutoDesk Eagle PCB software and ordered from Advanced Circuits. Five boards were ordered from Advanced Circuits, allowing for one "practice" board, given the exceedingly small size of some of the chosen components.

Figure 5.6 depicts the AutoDesk EagleCAD design of a single 8-channel multiplexer board, with red components representing the top routes, blue representing the bottom, and green representing the soldering pads. The circuit is designed to accept unregulated $\pm 7.5 \mathrm{~V}$ and ground input on the left side of the board. The $7.5 \mathrm{~V}$ unregulated power supply fed a $5 \mathrm{~V}$ Low-Dropout regulator (LDO), which was stabilized by the capacitors in positions $\mathrm{C} 2, \mathrm{C} 3, \mathrm{C} 6$, and $\mathrm{C} 7$, whose values are listed in Table 5.1, in addition to the diodes in positions D1 and D4. Similarly, the $-7.5 \mathrm{~V}$ unregulated power supply fed a $-5 \mathrm{~V}$ LDO, which was stabilized by the capacitors in positions $\mathrm{C} 4, \mathrm{C} 5, \mathrm{C} 6$, and $\mathrm{C} 7$ along with the diodes in positions D2 and D3. These two LDO circuits provided $\pm 5 \mathrm{~V}$ regulated power to the op-amps and mux chips. 


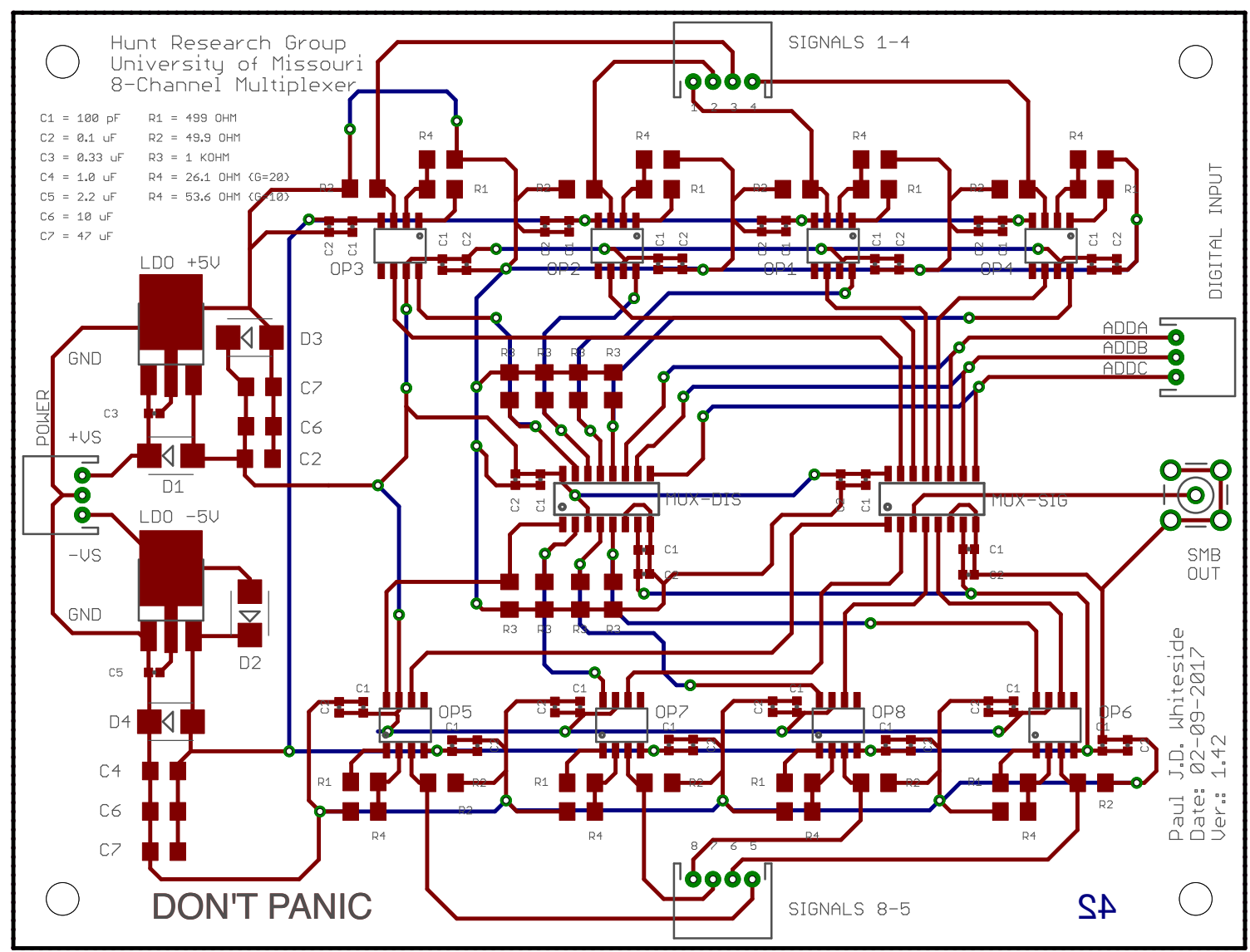

FIGURE 5.6 The multiplexer boards were designed to accept 8 signal inputs, to amplify and output 1 signal, and to shunt the other 7 to ground to reduce cross-talk between signals. The signals were input via two ribbon cable ports at the top and bottom, whereas power was provided via the three pin port on the left, and the multiplexer logic commands were provided from the three pin input port on the right.

The boards were designed to accept four input signals from the Imasonic array transducer at the top and bottom of the layout. Each of the signals was fed to an independent op-amp, which was designed as a non-inverting high-gain amplifier to improve the signal-to-noise ratio (SNR). The gain was defined by the ratio of resistors $\mathrm{R} 1$ and $\mathrm{R} 4$, which were the feedback $\left(R_{f}\right)$ and input $\left(R_{i}\right)$ resistors, respectively. The gain equation for such a non-inverting amplifier is shown in Equation 5.4 below:

$$
G=1+\frac{R_{f}}{R_{i}}
$$




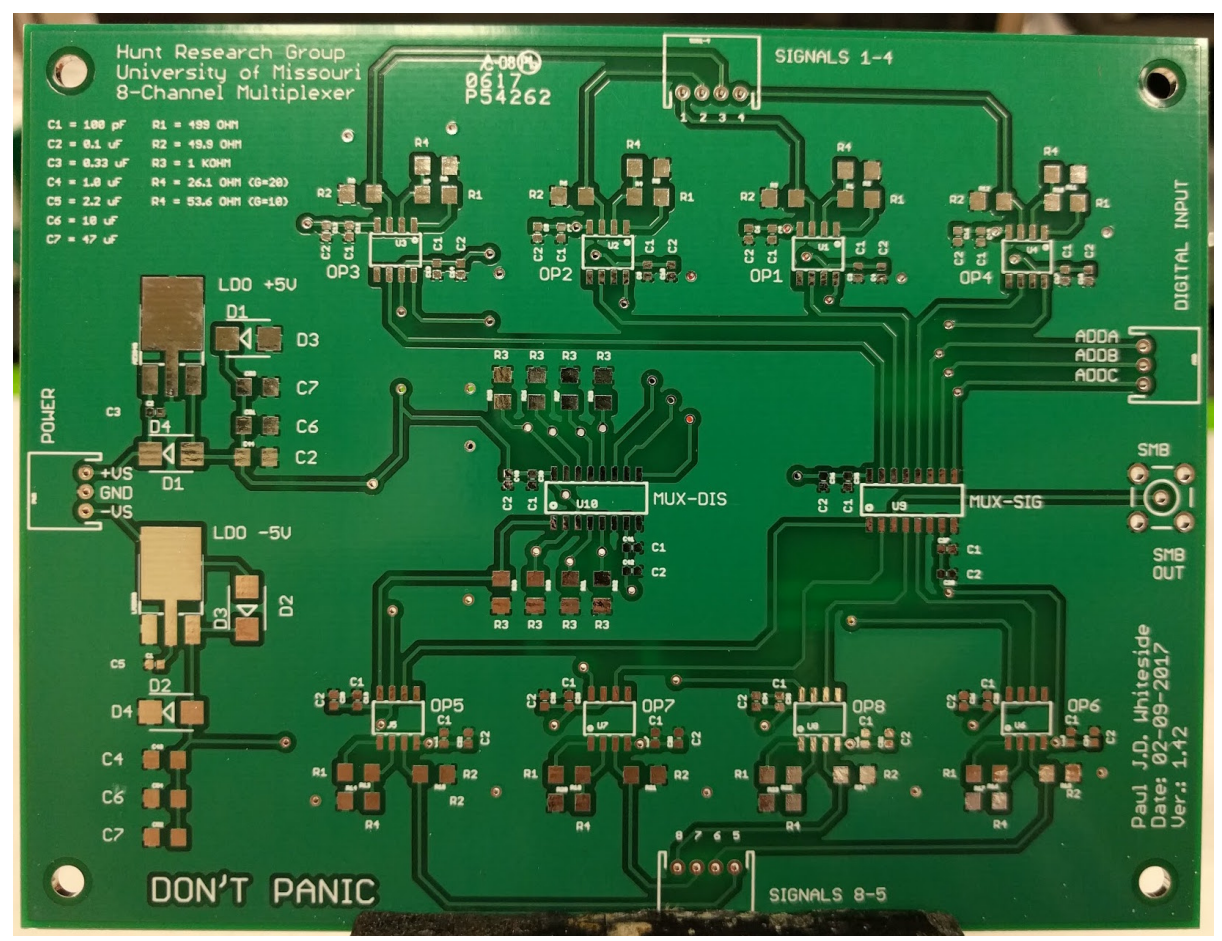

FIGURE 5.7 Stackable multiplexer boards were designed in EagleCAD to minimize high frequency interference, and were then printed by Advanced Circuits, LLC. The words of advice in the bottom left corner of the board are actually etched in copper as well and serve to aid the manufacturer in discerning between the files for the front and rear circuit traces.

As such, the gain could be set to either $G=20$ or $G=10$, depending on the choice of R4. It should be noted that the op-amp becomes less stable at high gains for higher frequency signals, so a lower gain should be selected if excessive ringing is observed in the output signal. Moreover, gains lower than 10 necessitate the incorporation of a compensation circuit, which may consist of a single capacitor or a capacitor and a resistor in series. The data sheet for the op-amps used for these multiplexer circuits recommended a compensation capacitor of $15 \mathrm{pF}$ for a gain of 10 , but did not require anything to be connected to the circuit for a gain of 20. Additionally, these particular op-amps incorporated a "disable" pin, which when deactivated would route the input signal to ground to minimize cross-talk between amplified signal channels within the mux chips. The op-amps and mux chips were all independently stabilized on both their positive and negative supply rails using 


\begin{tabular}{llll}
\hline \hline Symbol & Description & Manufacturer Part Number & Footprint \\
\hline LDO +5V & $5 \mathrm{~V}$ supply & AZ2940D-5.0TRE1 & TO-252-3 \\
LDO -5V & $-5 \mathrm{~V}$ supply & LM2990SX-5.0/NOPB & TO-263-3 \\
MUX-SIG & Signal output selection & MAX4051ESE+ & 16-SOIC \\
MUX-DIS & Disabling signals & MAX4051ESE+ & 16-SOIC \\
OP $(\#)$ & High gain op-amps & AD8099ARDZ-REEL7 & 8-SOIC \\
D $\#)$ & Diodes & RS3AB-13-F & DO-214AA \\
C1 & $100 \mathrm{pF}$ & CL05C101JC51PNC & 0402 \\
C2 & $0.1 \mu \mathrm{F}$ & GRM155R62A104KE14D & 0402 \\
C3 & $0.33 \mu \mathrm{F}$ & CL31B334KBFNNNE & 1206 \\
C4 & $1.0 \mu \mathrm{F}$ & CL31B105KCHNNNE & 1206 \\
C5 & $2.2 \mu \mathrm{F}$ & HMK316ABJ225KL-TE & 1206 \\
C6 & $10 \mu \mathrm{F}$ & UMK316BBJ106ML-T & 1206 \\
$\mathrm{C} 7$ & $47 \mu \mathrm{F}$ & C3216X5R1E476M160AC & 1206 \\
R1 & $499 \Omega$ & RC1206FR-07499RL & 1206 \\
R2 & $49.9 \Omega$ & RC1206FR-0749R9L & 1206 \\
R3 & $1 \mathrm{k} \Omega$ & RC1206FR-071KL & 1206 \\
R4 $(\mathrm{G}=20)$ & $26.1 \Omega$ & RC1206FR-0726R1L & 1206 \\
R4 $(\mathrm{G}=10)$ & $53.6 \Omega$ & ERJ-8ENF53R6V & 1206 \\
SMB & Molex right angle SMB & 731000103 & SMB \\
\hline \hline
\end{tabular}

TABLE 5.1 This table lists each of the components utilized in the creation of the 8-channel multiplexer PCB's shown in Figure 5.6. All components were purchased on Digikey.com

capacitors $\mathrm{C} 1$ and $\mathrm{C} 2$.

The output pins of each op-amp were fed to the 8-channel mux chip labeled "MUX-SIG," whereas their respective disable pins were fed to the mux chip labeled "MUX-DIS." The two mux chips were indexed by the three input pins labeled ADDA, ADDB, and ADDC. These digital indexes simultaneously controlled the output of MUX-SIG while also disabling the op-amps for each of the 7 unused signals through MUX-DIS. The digital address pins were the final input on the right side of the board and were designed for this case to be operated by an Arduino Mega 2560 microcontroller, which would in turn be operated by USB output from a LabVIEW automation software. Finally, the output of MUX-SIG was directed to a $50 \Omega$ output impedance Molex SMB right-angle connector. 


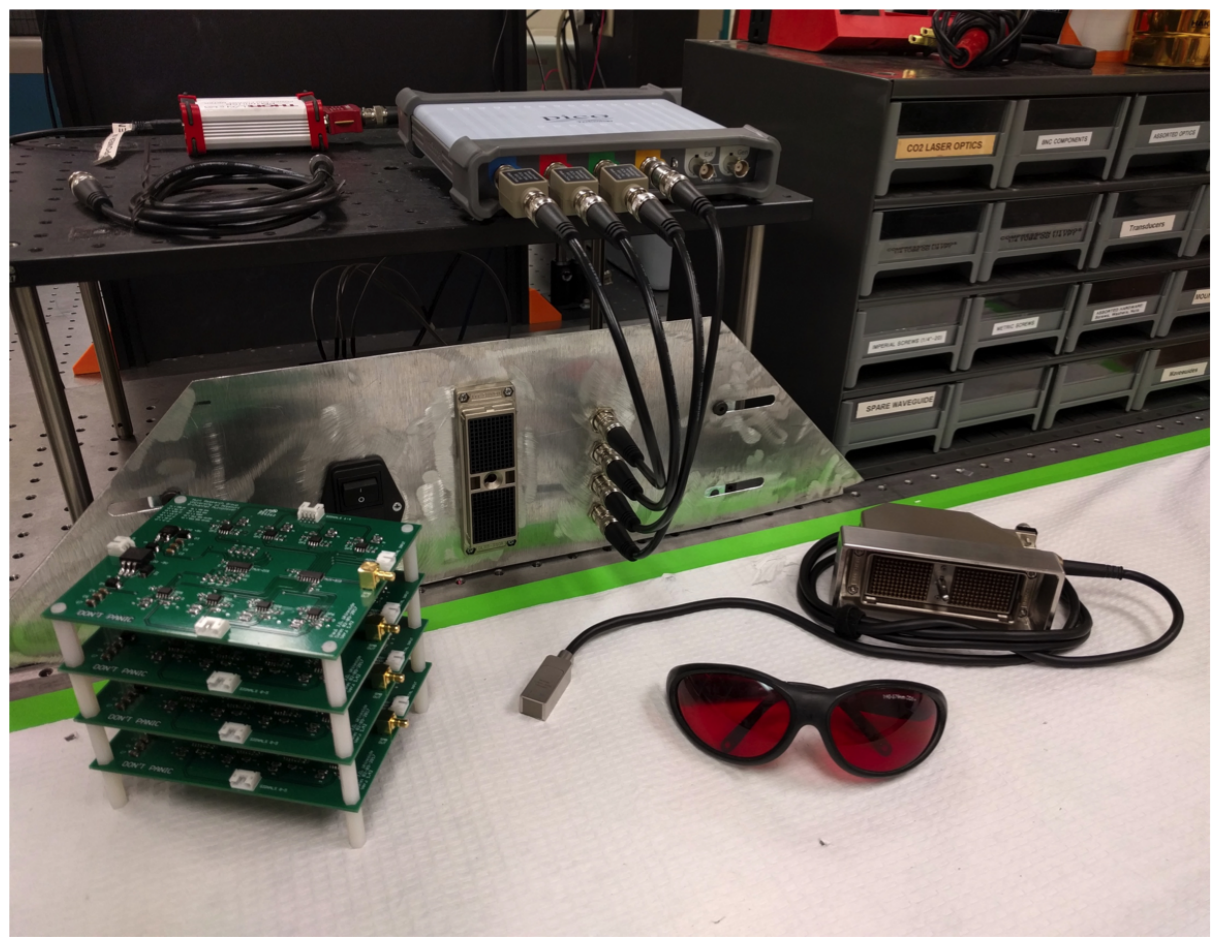

FigURE 5.8 The assembled multiplexer boards are stacked and fed unregulated $\pm 8 \mathrm{~V}$ and ground, 8 signal inputs each, and 3 arduino address signals. The multiplexed signals are output via SMB connectors to BNC front-panel ports, which then feed to the 4 input channels of the Picoscope 5442B, including $50 \Omega$ feedthrough terminators on each channel to minimize reflections.

Using four of these boards stacked on top of each other, as shown in Figure 5.8 , the 32 channels of the array transducer were separated into groups of 8 , isolated, amplified, and output to one of the four BNC input channels of the oscilloscope. Signals (1-8) corresponded to Channel A, (9-16) to Channel B, (17-24) to Channel C, and (25-32) to Channel D. Additionally, each of the BNC channels incorporated a $50 \Omega$ feedthrough terminator to more appropriately match the electrical impedance.

\subsection{Results}

As discussed previously, this project proceeded in two distinct phases, the first of which was intended to demonstrate proof of concept to show that backward-mode photoacoustics could be performed using selective release waveguides, whereas the second was intended to improve upon that system to generate a 2D depth profiling 
system for tomographic applications in a clinical setting. The first phase utilized porcine skin samples embedded with ABS plastic chromophores and demonstrates 1D photoacoustic depth profiling. The second phase utilized tissue phantoms that were specifically designed to be both optically and acoustically analogous to tissue, while permitting strong control over the position and depth of blood vessel analogues.

\subsubsection{Preliminary results}

To establish the groundwork for a backward-mode waveguide-based photoacoustic tomography modality, the initial phase of the project involved the generation and detection of photoacoustic signals within ex vivo samples of porcine skin, detecting the ultrasonic pulses on the reverse face of the transmitting waveguide. In that regard, experiments were designed such that the laser was fired 16 consecutive times, resulting in 16 waveforms that were averaged to improve signal to noise ratio (SNR). The light was transmitted into two tissue samples, one consisting of the original porcine tissue and the other consisting of porcine tissue with an ABS black plastic chromophore embedded in the back side of the dermis. The waveforms generated within each sample were compared to identify the sources of individual peaks and confirm that the signals from the ABS plastic chromophore could be successfully isolated and identified. To accomplish this, the position of each peak in the time domain was recorded and matched to the time of flight predictions based on the various paths and reflections that it might have taken, utilizing the speeds of sound in each of the materials involved $\left(c_{\text {acrylic }}=2730 \mathrm{~m} / \mathrm{s} ; c_{\text {gel }} \equiv c_{\text {water }}=1570 \mathrm{~m} / \mathrm{s} ; c_{\text {skin }}=1595 \mathrm{~m} / \mathrm{s}\right)$ to additively determine the total time of flight for each peak [206-208].

Figure 5.9 depicts the photoacoustic trace of the ABS-implanted tissue sample, and demonstrates the occurrence of six events of interest. The first two positive peaks, occurring at $3.942 \mu \mathrm{s}$ and $4.026 \mu \mathrm{s}$ were the closest to the transducer face and were determined to correlate with internal reflection points within the waveguide, 


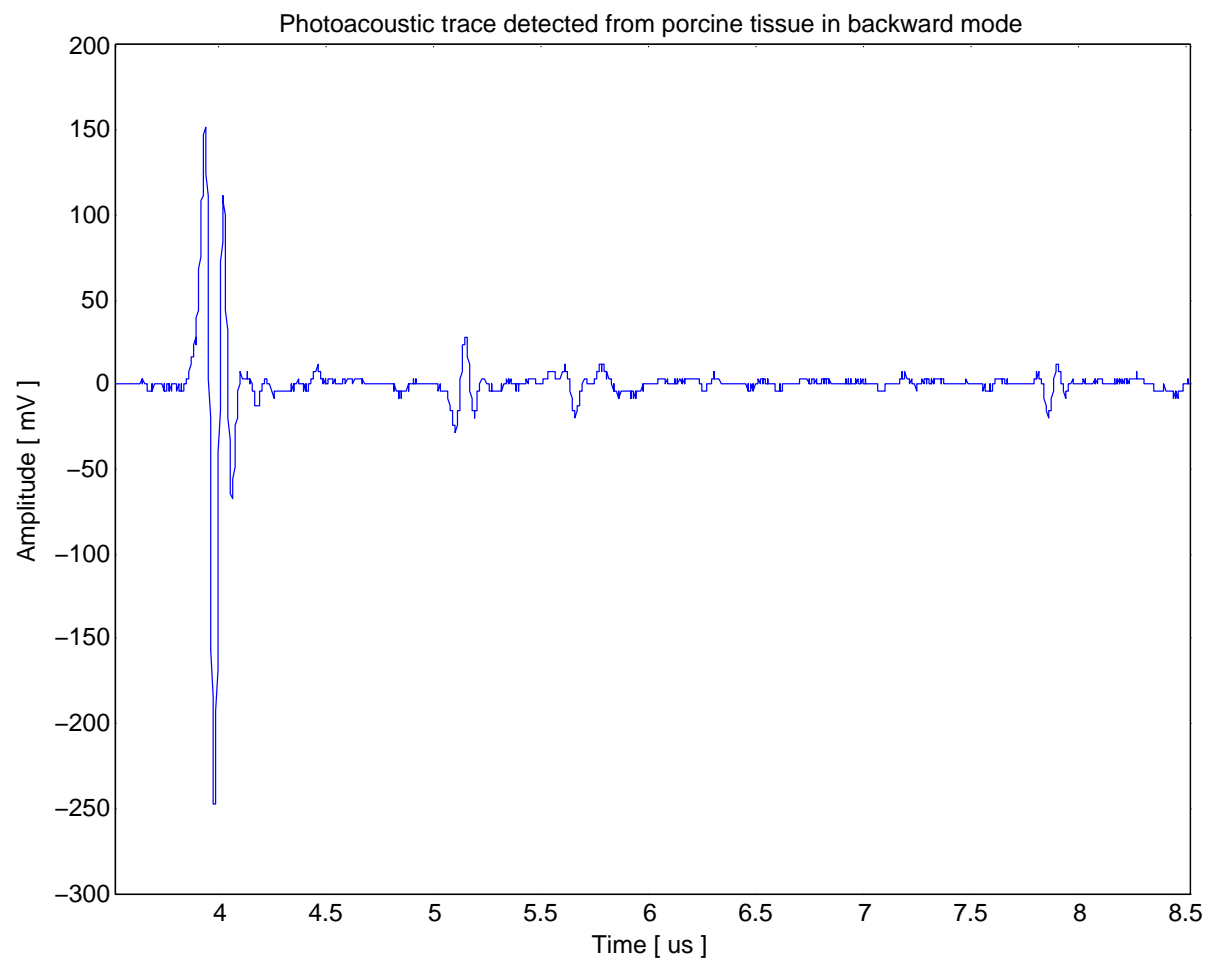

FIGURE 5.9 The photoacoustic peaks were analyzed to reveal that the most significant peaks were resultant from optical absorption by the silver cladding, which also generated acoustic reflections; these observations provided justification to redesign waveguide fabrication protocols to avoid using optically absorbing cladding layers.

suggesting that the silver cladding was producing a photoacoustic response. The two negative peaks occurring at $5.102 \mu \mathrm{s}$ and $5.192 \mu$ s represent the reflections of these signals from the waveguide tissue interface. Finally, the peaks occurring at $5.62 \mu \mathrm{s}$ and $7.86 \mu$ s correspond strongly with the known position of the ABS chromophore. Additionally, although the former peaks were present in the traces recorded from the unaltered porcine skin sample, the latter peaks were not, contributing to the assertion that these peaks were the result of optical absorption by the ABS chromophore.

\subsubsection{D Depth Profiling}

Having empirically proven that the backward mode technique to be a viable method of both transmitting light into ex vivo tissue and detecting the subsequent 
ultrasound, the project advanced to the second phase, wherein the single element transducer would be replaced by a 2D array of piezoelectric elements. Owing to the increased complexity of the acoustic apparatus, the initial 2D photoacoustic tomography experiments were designed to be performed first on well-controlled artificial tissue phantoms. The fabrication protocols for establishing appropriate phantoms to use in developing a bespoke photoacoustic imaging modality were refined experimentally to accurately replicate reported values of the speed of sound in tissues while also replicating appropriate optical scattering.

Although the tissue phantoms were designed based on a protocol published by Chen et al. , reproducing the protocol did not precisely reproduce the reported speed of sound in tissue [206]. Consequently, the phantom preparation protocols were iteratively revised to more closely match the speed of sound in tissue, resulting in the protocols listed in the previous section. After the speed of sound was first matched, five phantoms were produced to confirm the original analysis and the consistency with which the protocols would match the reported speed. The results of these tests are included in table 5.2 , which resulted in an average of $1562.048 \pm 8.425 \mathrm{~m} / \mathrm{s}$. This mean and standard deviation represent the consistency of calculated speed from one sample to the next; however, the deviation of calculated speeds within a single sample was only at most $3.243 \mathrm{~m} / \mathrm{s}$. The speed of sound in human dermis is reported to be $1595 \mathrm{~m} / \mathrm{s}$ and $1645 \mathrm{~m} / \mathrm{s}$ in human epidermis; consequently the average speed of sound is only $2.07 \%$ deviant from that in dermis and $5.04 \%$ from that in epidermis [192].

\subsection{Discussion}

The results of the first phase of this research project successfully demonstrated that backward-mode photoacoustic tomography could be performed using selective release waveguides to transmit light into the tissue with an acoustic detector on the rear face of the waveguide; however, they also revealed significant flaws in the 


\begin{tabular}{llll}
\hline \hline Sample & Speed $[\mathrm{m} / \mathrm{s}]$ & Average $[\mathrm{m} / \mathrm{s}]$ & Std. Dev. $[\mathrm{m} / \mathrm{s}]$ \\
\hline 1 (a) & 1572.515 & & \\
1 (b) & 1572.49 & 1573.247 & 1.289 \\
1 (c) & 1574.735 & & \\
2 (a) & 1566.58 & & \\
2 (b) & 1568.604 & 1568.618 & 2.045 \\
2 (c) & 1570.67 & & \\
3 (a) & 1548.82 & & \\
3 (b) & 1552.85 & 1551.393 & \\
3 (c) & 1552.51 & & \\
$4(\mathrm{a})$ & 1553.01 & & \\
4 (b) & 1558.94 & 1556.733 & \\
$4(\mathrm{c})$ & 1558.25 & & \\
5 (a) & 1557.77 & & \\
5 (b) & 1562.22 & 1560.247 & 2.267 \\
5 (c) & 1560.75 & & \\
\hline \hline
\end{tabular}

TABLE 5.2 The calculated speed of sound determined from three tests (a,b,c) in each of five tissue phantoms, resulting in an average $1562.048 \pm 8.425 \mathrm{~m} / \mathrm{s}$ for the speed of sound in the turbid media.

designs of the experimental apparatus and waveguides that compromise the reliability of such a system. Specifically, the results shown in Figure 5.9 revealed that the moderate optical absorption occurring within the silver cladding of the waveguides was generating multiple distinct photoacoustic signals. Because the signals occurred within the cladding they were exposed to the largest dose of laser energy, which resulted in spikes that were at least one order of magnitude greater than the signals of interest. Moreover, these signals were generated in relatively close proximity to the acoustic detection interface. This closeness permitted the associated acoustic reflections to reappear in the signal trace before the signals of interest, which poses significant concerns about the reliability of the system, as their magnitude is sufficient to overlap signals from the tissue.

We attempted to resolve these concerns by performing a frequency analysis of the system with no chromophore in the tissue, to hopefully identify the frequency 
response of the silver signals and potentially filter them out through a low-pass or high-pass digital LabView filter. However, our analysis revealed that there was not a distinct peak, but a broadband response that overlapped the typical frequency responses of biological chromophores, which have been observed to be between 5 and $15 \mathrm{MHz}$. As such, the waveguides have been redesigned for the second phase of the project to avoid the generation of such signals. Two solutions were posed for these designs, which will be investigated as the project continues. The first solution was to use a multi-layer thin film coating of alternating layers of $\mathrm{TiO}_{2}$ and $\mathrm{SiO}_{2}$, which are both transparent to $532 \mathrm{~nm}$. This solution is technically complex, but also permits the use of greater laser intensities. The second solution, as described in Section 5.3.3, are composed of multiple slabs of acrylic or polycarbonate that are adhered together using an optical adhesive, which is specifically chosen to have a lower refractive index than the substrate. The light is transmitted through the waveguide parallel to the tissue until it reaches the bonding interface, which is at an angle to the tissue, after which the light totally internally reflects toward the tissue. This approach is referred to as a planar beam deflector, rather than a traditional waveguide, and has distinct benefits in that the light now transmits perpendicularly though the tissue and does not generate the signals from any cladding layer. However, curing the optical adhesive may prove problematic as they are typically UV-curing but polycarbonate and acrylic substrates are non-transmissive to light lower than around $400 \mathrm{~nm}$. Additionally, reflections from the tissue surface may generate photoacoustic signals on the surface of the transducer. Another distinct benefit of this design is that it may permit multiple parallel interfaces to permit an array of transmitted beams to generate photoacoustic sources within the tissue, which may speed up analysis or improve the efficiency of live-imaging modalities.

With regard to the second phase of this project, at the time this dissertation was written, 2-dimensional photoacoustic tomographic data has not yet been collected. 
The tissue phantom protocols have been iteratively improved to generate tissue analogues that are optically and acoustically similar to human dermal tissue, as shown in Table 5.2, and the multiplexer boards are completely assembled, as shown in Figure 5.8, but the LabView program that will index the multiplexer and control signal acquisition from the 32 channels is still being written. Once the LabView program has been compiled, the system will control laser firing, index the 4 multiplexer boards to output 1 of 8 input signals each, and then compile all 32 channels into a single data file. That file may then be reconstructed using the Matlab k-wave analysis software toolbox, which uses four dimensional Fourier transform analysis to reconstruct tomographical depth profiles. Alternatively, LabView boasts an ultrasound analysis toolbox that uses traditional delay-and-sum beam-forming to analyze time of flight of signals and reproduce them into a $2 \mathrm{D}$ depth profile. Both methods have been used in photoacoustic tomography $[209,210,212]$. The $\mathrm{k}$-wave analysis is potentially more accurate, and readily allows for 3D analysis if a matrix array transducer is used or the laser beam is translated across the waveguide; however, the delay-and-sum system is more likely to permit live-imaging, which is arguably more valuable to clinicians. 


\section{Chapter 6}

\section{Conclusions \& continued investigations}

\subsection{Conclusions}

Contemporary laser-based medical procedures pose substantial problems with regard to ocular hazards, negative tissue side effects, and restrictions for the incorporation of auxiliary equipment. By contrast, the selective release waveguides discussed throughout this dissertation have been shown to resolve, mitigate, or otherwise circumvent each of the fundamental limitations of the traditional free-space method of laser transmission. The preliminary work performed and presented in the associated Master's Thesis project, as discussed in Section 1.4.1, successfully demonstrated that light could be transmitted into tissue phantoms by direct contact of an optical waveguide with the target medium. Moreover, the work established that the transmission could be regulated in part through control of the composition and thickness of active area optical thin films.

That preliminary work has since been refined through this dissertation and shown to be applicable in a variety of disparate methods. The selective release waveguides discussed in Chapter 3 further confirm the principles of contact-based transmission and regulation of that transmission at each individual active area reflection point by varying local film thickness. Results demonstrate that transmission decreases substantially with even just $60 \mathrm{~nm}$ of silver in the active area. Additionally, 
the results suggest that the active area thickness must be closely controlled within a margin of error of less than $10 \mathrm{~nm}$, as transmission decreased significantly at that increment beyond a $30 \mathrm{~nm}$ thickness.

The selective release waveguides were also put into use in Chapter 4 to demonstrate the incorporation of an auxiliary technology in order to improve potential therapeutic effects. This research led to the observation of a new phenomenon in the form of sonoillumination, wherein tissue optical properties are modulated using continuously pulsed ultrasound throughout the therapeutic laser treatment. This combination of phenomena resulted in a $174 \%$ increase in forward transmission, which has significant ramifications with regard to treatment efficacy and the mitigation of detrimental photothermal side effects within the tissue. Moreover, the increase in transmission may also permit the broadening of laser-based treatments to patients of darker complexions by decreasing the relative absorption of light by epidermal melanin. However, this project concretely established the principle of combining two distinct technologies simultaneously during a laser procedure without need for altering the auxiliary equipment in any manner.

Finally, the method of backward-mode photoacoustic tomography discussed in Chapter 5 further demonstrates the applicability of auxiliary equipment with selective release waveguides by transmitting light into the tissue through contact and subsequently detecting the photoacoustically generated ultrasonic signals. The project also highlighted some unanticipated problems that need to be resolved before the technology can be applied in a clinical setting. Specifically, the results demonstrated significant absorption in the cladding of the waveguides, which resulted in overwhelming photoacoustic signals that had the potential to overlap meaningful signals produced within the tissue. Although not as remarkably successful as the sonoillumination project, it laid the foundation for continued research to establish a clinical imaging modality based on the photoacoustic effect that has the potential to address substan- 
tial needs in such areas as the diagnosis and mapping of severe burns, pressure ulcers, and capillary perfusion into skin grafts.

\subsection{Continued investigations}

Although the work presented herein has established proof of concept for each of the respective projects, there are some significant hurdles to overcome before the systems will see commercial or clinical application. The following final sections discuss some of my ideas about how the projects should continue.

\subsubsection{Project: Selective-release waveguides}

The selective release waveguides successfully regulated transmission of laser pulses through the tissue samples in ex vivo testing, however, before pre-clinical and clinical testing can begin, the design of the waveguides should be revised slightly. Specifically, the silver cladding, while providing almost complete total internal reflection regardless of internal reflection angle is slightly optically absorbing. At clinically relevant energies between 4 and $12 \mathrm{~J} / \mathrm{cm}^{2}$, the silver cladding may become damaged, particularly with prolonged exposure. I would therefore recommend investigating the use of multi-layer thin film claddings instead, as are used in anti-reflection coatings, but constructed in such a way that the light totally internally reflects at the defined internal reflection angle. Typical coatings are composed of alternating layers of materials such as $\mathrm{TiO}_{2}$ and $\mathrm{SiO}_{2}$, neither of which is optically absorbing in the visible spectrum Such coatings can reliably achieve damage thresholds above $5 \mathrm{~J} / \mathrm{cm}^{2}$. The multi-layer thin film coatings also boast the benefit of being dichroic and partially transmissive to light beyond the specific wavelength and incident angle, as demonstrated in Figure 6.1, which shows a sample waveguide I had designed with alternating layers of $\mathrm{TiO}_{2}$ and $\mathrm{SiO}_{2}$ in $49.8 \mathrm{~nm}$ and $91.1 \mathrm{~nm}$ thicknesses respectfully, for a total of 5 layer pairs. The coating resulted in partial reflection, but allowed substantial light transmission; however, I could not identify whether the films had not been deposited 


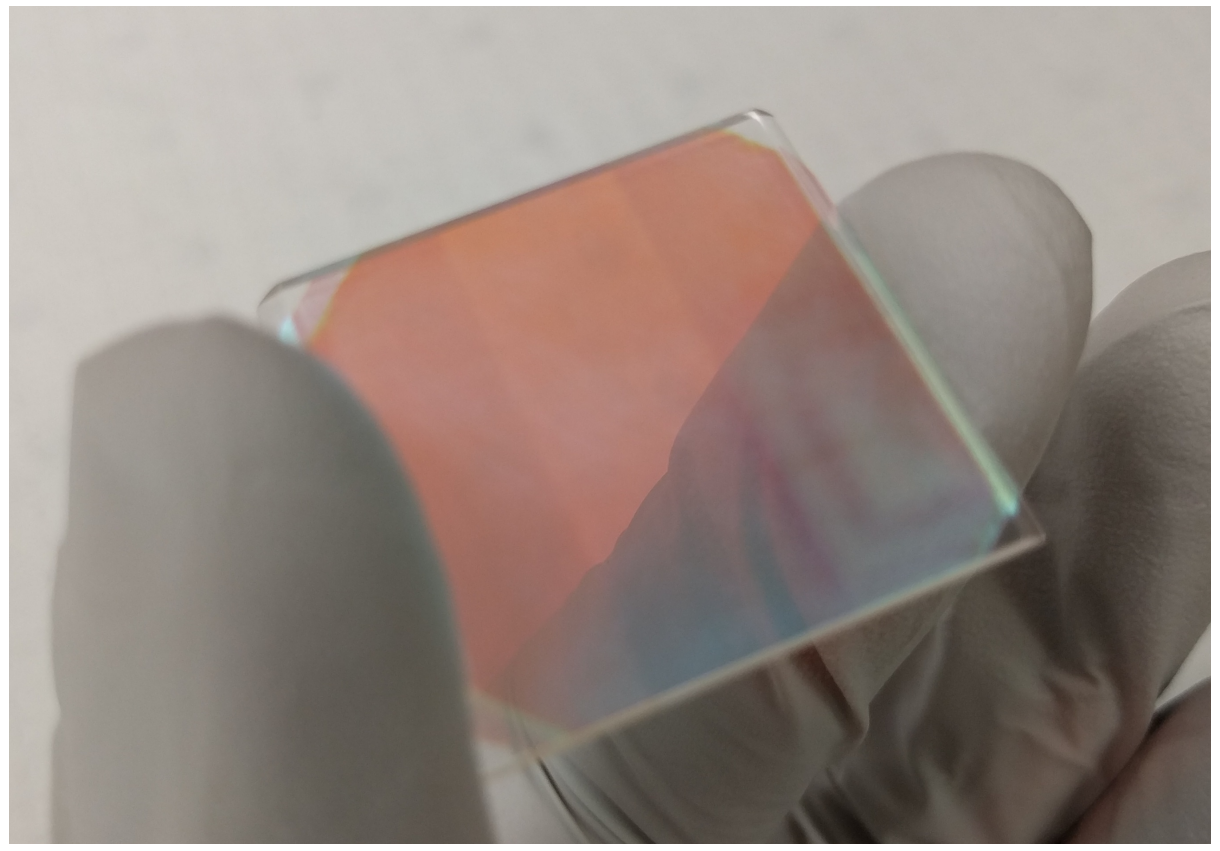

FIGURE 6.1 A waveguide with a dichroic multi-layer thin film cladding designed to totally internally reflect $532 \mathrm{~nm}$ laser light at $45^{\circ}$ incidence, which would permit operation at a greater optical damage threshold than silver layers do.

correctly, or the film thicknesses were inappropriate. I recommend having a professional optical coatings company deposit such films in the future. Nevertheless, even the coating we achieved performed as a dichroic layer, with the figure showing that the active area and glove can be discerned through the waveguide cladding, which may permit clinicians to have a "viewing window" during laser procedures.

In addition to the non-absorptive cladding change, the improved safety of the SRW design over free-space propagation methods must be empirically established though measurement of the reflected intensity. Although it seems obvious that enclosing the optical system would inhibit back-reflections, confirming this claim with evidence based analysis may aid in attracting industry collaborations. I recommend moving the integrating sphere to the rear face of the waveguide and blocking off the surrounding area with a highly reflective white scattering plastic. Compare this against the free-space modality in terms of reflected intensity detected by a photodiode attached to the integrating sphere. It may not need to be completely enclosed, 
due to the significant decrease in comparative measurements; however, it may be possible to reduce light escaping from the system by transmitting the free-space beam off a mirrored prism attached to the entrance port of the integrating sphere. Once these two concerns have been addressed, the project can advance to testing with clinically relevant energies and transition into the development of a hand-piece prototype that can be adapted for use with a clinical laser system.

\subsubsection{Project: Sonoillumination}

Although the sonoillumination experiments demonstrated remarkable success in not only improving transmission through simultaneous ultrasonic modulation of tissue, but also in demonstrating the applicability of auxiliary technologies alongside laser irradiation, the phenomenon of sonoillumination is still not fully understood. The phenomenological model predicts that there are thresholds of operation that separate distinct mechanistic regimes. As such, I would recommend first performing a thorough analysis of the relationship between acoustic pulsation magnitude and transmitted optical intensity. Specifically, future experiments should establish a continuous curve relating increasing amplitude to optical transmission to demonstrate increasing regions, plateaus, and regions exhibiting a physiological change in the medium through sonophoresis. These tests would be better related to acoustic pressure than driving voltage, and so I would also recommend determining the conversion efficiency of the transducer to determine acoustic pressure as related to electronic pulsation amplitude, which may be accomplished with a radiation force balance, although it may be possible to request that the manufacturer of the acoustic probe characterize the transducer in advance. If the latter route is chosen, the efficiency of the electrical impedance matching circuit should also be taken into account. Electrical impedance is also better matched using transformer circuits than the L-match circuit used herein. 


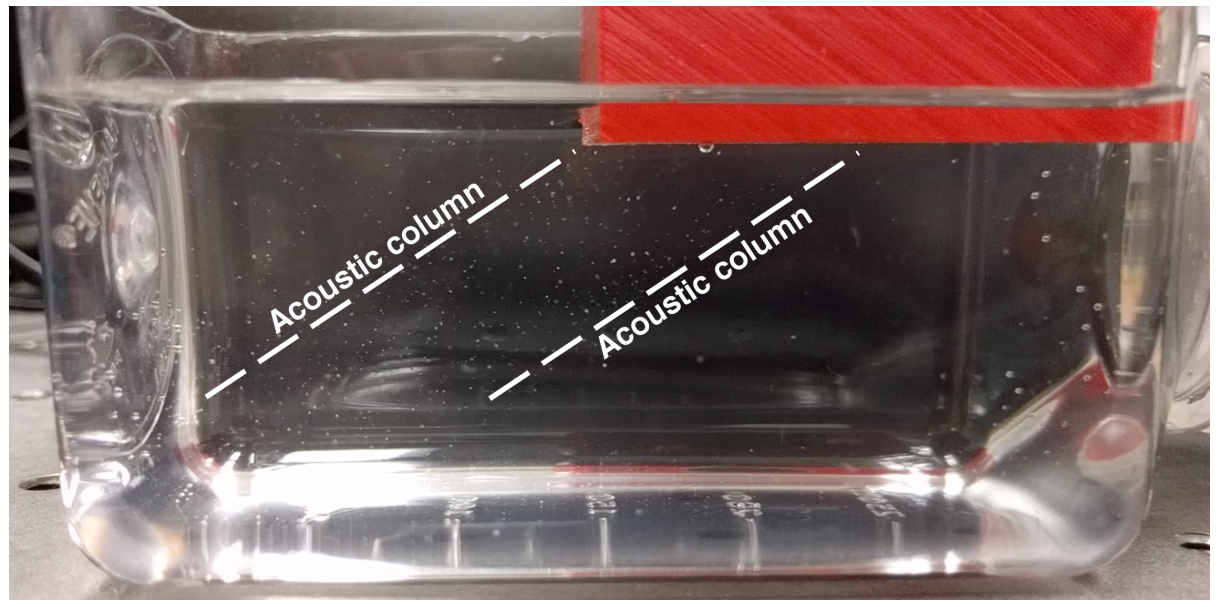

Figure 6.2 A mold for an acoustic wedge was 3D printed and filled with a silicone elastomer to transmit continuously pulsed $510 \mathrm{kHz}$ ultrasound into a water bath to generate acoustically-induced cavities within the water.

In addition to varying the magnitude, a range of frequencies and angles between propagation vectors should also be investigated. With regard to frequencies, I would recommend testing pulsation frequencies below, within, and at the predicted primary and secondary threshold frequencies using comparable acoustic pressures. Each transducer will likely necessitate its own impedance matching circuit and would benefit from being characterized by a radiation force balance (RFB). Additionally, if an RFB is available, each transducer may be pulsed within it's $-6 \mathrm{~dB}$ bandwidth, rather than exclusively at the central frequency, to gain a wider array of considered frequencies; however, the conversion efficiency will decrease as the frequency deviates from the central frequency of the transducer.

With regard to the angle between propagation vectors, it may be possible to apply ultrasound at an angle to the tissue for use in standard free-space laser transmission, rather than exclusively using an SRW. This may be performed using an acoustic wedge composed of a material whose acoustic impedance and attenuation characteristics are similar to those of tissue. Thus far I have attempted to use a silicone elastomer to form an acoustic wedge and managed to transmit an ultrasonic column into a water bath at an angle, as shown in Figure 6.2, but have yet to demon- 
strate the effect in tissue. The acoustic wedge refracted the ultrasound into the water and generated a column of sonophoretically induced bubbles that were held in vibrational stasis as long as ultrasound was being applied. That wedge had been designed to be placed such that the edge of the $3 \mathrm{D}$ printed mold would align with either a free-space beam or a waveguide-mediated beam, such that the acoustic pressure refracts underneath the incident beam; however, an improved design might incorporate an extension onto the wedge such that the light and ultrasound impact the tissue at the same location for greater effect. Moreover, it may be advisable to investigate using a focused transducer with the focal point located at the surface of the tissue for maximum epidermal effect.

\subsubsection{Project: Waveguide-mediated PAT}

With regard to the continuation of the photoacoustic tomography work, the project is already on course to transition into the next stage of testing. At the time of this dissertation, the multiplexer boards have been completed and the next steps include programming LabView to control the 32 channel detection system and output the recorded data into a single file. At that point, the $2 \mathrm{D}$ reconstruction may be performed using the aforementioned k-wave toolbox in Matlab or the delay-andsum beam-forming toolbox in LabView. Regardless which method is chosen, they will both require slight modification to account for the thickness and acoustic properties of the waveguide substrate when calculating reconstructions. It may be advisable to measure these properties in lab by producing a photoacoustic signal on one side of the substrate and measuring it on the other. Then compare that time-of-flight analysis with a similar system, using DI water in place of the substrate to confirm that the analysis matches the known speed of sound in water.

Once the 2D reconstruction software has been programmed, the project should first confirm the accuracy of the system using tissue phantoms with blood analogues 
(e.g. wires or tubes filled with absorbing dye solutions). Following that, the research should transition to ex vivo porcine skin and thermally damaged porcine skin. Heating a circular metal rod and placing it against the skin for a controlled amount of time should induce a change in the mechanical properties of the tissue, which should be observable using photoacoustics. However, ex vivo tissue will not exhibit the coagulative or inflammatory responses that would be observed in vivo, so the logical progression would be to transition from laboratory bench-top systems to pre-clinical trials with hairless pig or hamster models. Alternatively, the photoacoustic system may be used to image tattooed ex vivo porcine skin to demonstrate the accuracy and resolution of the system.

It may also be possible to generate 3D images by compiling $2 \mathrm{D}$ depth profiles produced by scanning a small spot size laser beam across the active area of the waveguide. The amplitude of the photoacoustic signal is proportional to the radiant exposure of the beam, $H_{0}$, so the use of a smaller spot size beam may be advantageous even for 2D profiling. However, when using a smaller beam spot, particular care must be taken to avoid exceeding the optical damage threshold of the optics. Operating with a safety factor of $1 / 3$ or $1 / 5$ of the published damage threshold is typical, which helps to account for pulse to pulse energy variations. Additionally, when designing a stand-alone backward-mode photoacoustic tomography system for any pre-clinical or clinical studies, the choice of laser should be based on the available wavelengths, the pulse width, and the maximum energy; however, selecting the highest energy laser may actually reduce the reliability of the system. Pulsed laser systems operate most consistently near their maximum energy. Consequently, if the maximum output energy is $50 \mathrm{~mJ}$ but the system only requires $5 \mathrm{~mJ}$, the laser will behave less reliably and will introduce greater errors into the data. 


\section{Appendix A}

\section{Derivation of Theoretical Equations}

\section{A.1 Maxwell's Equations}

$$
\begin{array}{r}
\nabla \times \boldsymbol{H}=j+\frac{\partial D}{\partial t} \\
\nabla \times \boldsymbol{E}=-\frac{\partial B}{\partial t} \\
\nabla \cdot D=\rho \\
\nabla \cdot B=0 \\
j=\sigma \boldsymbol{E} \\
D=\varepsilon \boldsymbol{E} \\
B=\mu \boldsymbol{H} \\
\varepsilon=\varepsilon_{r} \varepsilon_{0} \\
\mu=\mu_{r} \mu_{0}
\end{array}
$$

$\boldsymbol{H}=$ Magnetic field strength

$B=$ Magnetic flux density

$\boldsymbol{E}=$ Electric field strength

$D=$ Electric displacement

$j=$ Electric current density

$\rho=$ Electric charge density

$\sigma=$ Electric conductivity

$\mu=$ permeability

$\varepsilon=$ permittivity

$\varepsilon_{0}=$ permittivity of free space

$\mu_{0}=$ permeability of free space

$\varepsilon_{r}=$ relative permittivity

$\mu_{r}=$ relative permability

$c=$ speed of light in a vacuum

\section{A.1.1 Wave Equation in terms of $E$}

In order to get an expression for the wave equation in terms of the Electric Field, $\boldsymbol{E}$, all of the magnetic components $(\boldsymbol{H}$ and $B)$ must be replaced with their electric equivalents. The following rigorous derivation demonstrates this process step by step, until reaching the general expression for the wave equation in terms of $\boldsymbol{E}$ (Eq. A.8). In the derivation below and those that follow, equations of some significance 
are accompanied by numerical tags for reference purposes; whereas intermediate steps are accompanied by a brief note describing the action required to proceed from the previous step, included on the right of each line.

$$
\begin{array}{rlr}
\nabla \times \boldsymbol{H} & =j+\frac{\partial D}{\partial t} & \text { (From Eq. A.1) } \\
& =\sigma \boldsymbol{E}+\varepsilon \frac{\partial \boldsymbol{E}}{\partial t} & \text { (Substitute } D=\varepsilon \boldsymbol{E}, j=\sigma \boldsymbol{E}) \\
\nabla \times\left(\frac{1}{\mu} B\right) & =\sigma \boldsymbol{E}+\varepsilon \frac{\partial \boldsymbol{E}}{\partial t} & \text { (Substitute } B=\mu \boldsymbol{H}) \\
\frac{1}{\mu}(\nabla \times B) & =\sigma \boldsymbol{E}+\varepsilon \frac{\partial \boldsymbol{E}}{\partial t} & \text { (Factor in the Curl of } B) \\
\therefore \nabla \times B & =\mu \sigma \boldsymbol{E}+\mu \varepsilon \frac{\partial \boldsymbol{E}}{\partial t} &
\end{array}
$$

$$
\begin{array}{rlr}
\nabla \cdot D & =0 \\
& =\nabla \cdot(\varepsilon \boldsymbol{E}) \\
& =\varepsilon(\nabla \cdot \boldsymbol{E}) & (\text { Charge density in a vacuum is } 0) \\
(\text { Substitute } D=\varepsilon \boldsymbol{E})
\end{array}
$$

The expressions derived in Equations A.5 and A.6 will be utilized in the subsequent derivation, but are presented at the start of the process for ease of reference. The derivation of the wave equation will henceforth proceed unimpeded until the complete expression has been found. 


$$
\begin{array}{rlrl}
\nabla \times(\nabla \times \boldsymbol{E}) & =\nabla \times\left(-\frac{\partial B}{\partial t}\right) & & \text { (Take the curl of Eq. A.2) } \\
& =-\frac{\partial}{\partial t}(\nabla \times B) & \text { (Factor in the Curl of } B) \\
\nabla(\nabla \cdot \boldsymbol{E})-\nabla^{2} \boldsymbol{E} & =-\frac{\partial}{\partial t}(\nabla \times B) & & \text { (Curl of the Curl identity) } \\
0-\nabla^{2} \boldsymbol{E} & =-\frac{\partial}{\partial t}(\nabla \times B) & \\
\therefore \nabla^{2} \boldsymbol{E} & =\frac{\partial}{\partial t}(\nabla \times B) & \text { (Substitute in Eq. A.6) } \\
\nabla^{2} \boldsymbol{E} & =\frac{\partial}{\partial t}\left(\mu \sigma \boldsymbol{E}+\mu \varepsilon \frac{\partial \boldsymbol{E}}{\partial t}\right) \\
& =\mu \sigma \frac{\partial \boldsymbol{E}}{\partial t}+\mu \sigma \frac{\partial^{2} \boldsymbol{E}}{\partial t^{2}} & \text { (A.7) } \\
\therefore \nabla^{2} \boldsymbol{E} & =\mu \sigma \frac{\partial \boldsymbol{E}}{\partial t}+\mu \varepsilon \frac{\partial^{2} \boldsymbol{E}}{\partial t^{2}} & \text { (Subtitute in Eq. A.5) }
\end{array}
$$

One solution of Eq. A.8 is in the complex form of a plane-polarized harmonic plane wave:

$$
\begin{aligned}
& \qquad \boldsymbol{E}=\mathcal{E} \exp \left[\mathrm{i} \omega\left(t-\frac{x}{v}\right)\right] \\
& \text { with the condition that: } \frac{\omega^{2}}{v^{2}}=\omega^{2} \varepsilon \mu-\mathrm{i} \omega \mu \sigma
\end{aligned}
$$

In this representation, $\mathcal{E}$ represents the vector amplitude for the Electric wave. The complex representation of the refractive index $N=n-\mathrm{i} \kappa$, where $n$ and $\kappa$ are the real and complex components of the refractive index respectively, can then be readily derived from Eq. A.10, and subsequently a much more useful form of the plane wave equation. 


$$
\begin{aligned}
& \frac{\omega^{2}}{c^{2}}=\omega^{2} \varepsilon \mu-\mathrm{i} \omega \mu \sigma \quad \quad(v=c \text { in a vacuum }) \\
& =\omega^{2} \varepsilon \mu-0 \quad(\sigma=0 \text { in a vacuum }) \\
& =\omega^{2} \varepsilon_{0} \mu_{0} \quad \text { (Use } \varepsilon_{0} \text { and } \mu_{0} \text { for a vacuum) } \\
& \therefore c^{2}=\frac{1}{\varepsilon_{0} \mu_{0}} \\
& c^{2} \frac{\omega^{2}}{v^{2}}=\left(\omega^{2} \varepsilon \mu-\mathrm{i} \omega \mu \sigma\right) \frac{1}{\varepsilon_{0} \mu_{0}} \quad \text { (Multiply Eq. A.10 by Eq. A.11) } \\
& \frac{c^{2}}{v^{2}}=\frac{\varepsilon \mu}{\varepsilon_{0} \mu_{0}}-\mathrm{i} \frac{\mu \sigma}{\omega \varepsilon_{0} \mu_{0}} \quad \text { (Divide through by } \omega^{2} \text { ) } \\
& \left.=\varepsilon_{r} \mu_{r}-\mathrm{i} \frac{\mu_{r} \sigma}{\varepsilon_{0} \omega} \quad \text { (Substitute } \varepsilon=\varepsilon_{r} \varepsilon_{0} \text { and } \mu=\mu_{r} \mu_{0}\right) \\
& \therefore \frac{c^{2}}{v^{2}}=\varepsilon_{r} \mu_{r}-\mathrm{i} \frac{\mu_{r} \sigma}{\varepsilon_{0} \omega} \\
& \left.\frac{c^{2}}{v^{2}}=N^{2} \quad \text { (Define } N={ }^{c} / v\right) \\
& N^{2}=(n-\mathrm{i} k)^{2} \quad(\mathrm{~N} \text { is of the form } n-\mathrm{i} k) \\
& =n^{2}-\mathrm{i} 2 n k-k^{2} \quad \text { (Factor the exponential expression) } \\
& =\left(n^{2}-k^{2}\right)-\mathrm{i} 2 n k \quad \text { (Group like terms for comparison) } \\
& \left(n^{2}-k^{2}\right)-\mathrm{i} 2 n k=\varepsilon_{r} \mu_{r}-\mathrm{i} \frac{\mu_{r} \sigma}{\varepsilon_{0} \omega} \\
& \therefore\left(n^{2}-k^{2}\right)=\epsilon_{r} \mu_{r} \\
& \therefore 2 n k=\frac{\mu_{r} \sigma}{\varepsilon_{0} \omega}
\end{aligned}
$$

Equation A.8 can then be rewritten to incorporate some more useful elements like the complex refractive index term $N$ and the wavelength term $\lambda$. Subsequently, the equation can be generalized for any plane-polarized wave propagating in the direction given by unit vector $\hat{s}=\langle\alpha i+\beta j+\gamma k\rangle$, as shown below: 


$$
\begin{aligned}
\boldsymbol{E} & =\mathcal{E} \exp \left[\mathrm{i}\left(\omega t-\frac{\omega N x}{c}\right)\right] \quad \quad \text { (Substitute } v=\frac{c}{N} \text { into Eq. A.8) } \\
& =\mathcal{E} \exp \left[\mathrm{i}\left(\omega t-\left(\frac{2 \pi N}{\lambda}\right) x\right)\right] \\
\therefore \boldsymbol{E} & =\mathcal{E} \exp \left[\mathrm{i}\left(\omega t-\left(\frac{2 \pi N}{\lambda}\right)(\alpha x+\beta j+\gamma z)\right)\right]
\end{aligned}
$$

\section{A.1.2 Wave Equation in terms of $\boldsymbol{H}$}

A similar expression can be derived for the Wave Equation in terms of the Magnetic Field , $\boldsymbol{H}$. Much of the derivation is identical to that which led to Eq. A.15, but the portions that differ are shown below. Again, Equation A.16 is included for use later on in the derivation of the wave equation, but is included at the start for ease of reference.

$$
\begin{aligned}
\nabla \cdot B & =0 \\
& =\nabla \cdot(\mu \boldsymbol{H}) \\
& =\mu(\nabla \cdot \boldsymbol{H}) \\
\therefore \nabla \cdot \boldsymbol{H} & =0
\end{aligned}
$$




$$
\begin{array}{rlrl}
\nabla \times(\nabla \times \boldsymbol{H}) & =\nabla \times\left(j+\frac{\partial D}{\partial t}\right) & \text { (Take the curl of Eq. A.1) } \\
& =\sigma(\nabla \times \boldsymbol{E})+\varepsilon \frac{\partial}{\partial t}(\nabla \times \boldsymbol{E}) & & \text { (Substitute } j=\sigma \boldsymbol{E}, D=\varepsilon \boldsymbol{E}) \\
& =-\sigma \frac{\partial B}{\partial t}-\varepsilon \frac{\partial^{2} B}{\partial t^{2}} & \text { (Substitute Eq. A.2 for } \nabla \times \boldsymbol{E}) \\
& =-\varepsilon \mu \frac{\partial^{2} \boldsymbol{H}}{\partial t^{2}}-\sigma \mu \frac{\partial \boldsymbol{H}}{\partial t} & \text { (Substitute } B=\mu \boldsymbol{H}) \\
\nabla(\nabla \cdot \boldsymbol{H})-\nabla^{2} \boldsymbol{H} & =-\varepsilon \mu \frac{\partial^{2} \boldsymbol{H}}{\partial t^{2}}-\sigma \mu \frac{\partial \boldsymbol{H}}{\partial t} & \text { (Curl of the Curl identity) } \\
0-\nabla^{2} \boldsymbol{H} & =-\varepsilon \mu \frac{\partial^{2} \boldsymbol{H}}{\partial t^{2}}-\sigma \mu \frac{\partial \boldsymbol{H}}{\partial t} & \text { (Substitute in Eq. A.16) } \\
\therefore \nabla^{2} \boldsymbol{H} & =\varepsilon \mu \frac{\partial^{2} \boldsymbol{H}}{\partial t^{2}}+\sigma \mu \frac{\partial \boldsymbol{H}}{\partial t} & \text { (A.17) }
\end{array}
$$

One solution of Eq. A.17 is in the complex form of a plane-polarized harmonic

plane wave, as was the case in the derivation of the Wave Equation in terms of $\boldsymbol{E}$. A nearly identical equation results from the derivation of the Wave Equation in terms of $\mathrm{H}$, where $\mathcal{H}$ represents the vector amplitude for the magnetic wave:

$$
\boldsymbol{H}=\mathcal{H} \exp \left[\mathrm{i}\left(\omega t-\left(\frac{2 \pi N}{\lambda}\right)(\alpha x+\beta j+\gamma z)\right)\right]
$$

\section{A.1.3 Characteristic Optical Admittance, $\mathcal{Y}$}

The following derivation utilizes portions of sections A.1.1 and A.1.2 in order to gain a relation between $\boldsymbol{E}$ and $\boldsymbol{H}$ in terms of the characteristic optical admittance $\mathcal{Y}$. Following from Eq. A.15, we first gain an expression for the curl of $\boldsymbol{H}$ in terms of $\boldsymbol{E}$, before finding an alternate expression for the curl of $\boldsymbol{H}$ that suits more appropriately suits our intentions. 


$$
\begin{aligned}
\frac{\partial}{\partial t} \boldsymbol{E} & =\mathrm{i} \omega \mathcal{E} \exp \left[\mathrm{i}\left(\omega t-\left(\frac{2 \pi N}{\lambda}\right)(\alpha x+\beta j+\gamma z)\right)\right] \\
& =\mathrm{i} \omega \boldsymbol{E}
\end{aligned}
$$

$$
\begin{aligned}
\nabla \times \boldsymbol{H} & =j+\frac{\partial D}{\partial t} \\
& =\sigma \boldsymbol{E}+\varepsilon \frac{\partial \boldsymbol{E}}{\partial t} \\
& =\sigma \boldsymbol{E}+\varepsilon(\mathrm{i} \omega \boldsymbol{E}) \\
& =(\sigma+\mathrm{i} \omega \varepsilon) \boldsymbol{E} \\
& =(\sigma+\mathrm{i} \omega \varepsilon) \frac{N^{2}}{\varepsilon_{r} \mu_{r}-\mathrm{i} \frac{\mu_{r} \sigma}{\omega \varepsilon_{0}}} \boldsymbol{E} \\
& =(\sigma+\mathrm{i} \omega \varepsilon) \frac{N^{2}}{\frac{\varepsilon \mu}{\varepsilon_{0} \mu_{0}}-\mathrm{i} \frac{\mu \sigma}{\omega \varepsilon_{0} \mu_{0}}} \boldsymbol{E} \\
& =(\sigma+\mathrm{i} \omega \varepsilon) \frac{N^{2}}{\frac{\varepsilon \mu \omega-\mathrm{i} \mu \sigma}{\varepsilon_{0} \mu_{0} \omega}} \boldsymbol{E} \\
& =\frac{(\sigma+\mathrm{i} \omega \varepsilon) N^{2} \omega}{c^{2}(\varepsilon \mu \omega-\mathrm{i} \mu \sigma)} \boldsymbol{E} \\
& =\frac{\mathrm{i}(\sigma+\mathrm{i} \omega \varepsilon) N^{2} \omega}{\mathrm{i}} \frac{\mathrm{c}}{c^{2} \mu(\varepsilon \omega-\mathrm{i} \sigma)} \\
& =\frac{\mathrm{i}(\sigma+\mathrm{i} \omega \varepsilon) N^{2} \omega}{c^{2} \mu(\sigma+\mathrm{i} \omega \varepsilon)} \boldsymbol{E} \\
\therefore \times \boldsymbol{H} & =\mathrm{i} \frac{N^{2} \omega}{c^{2} \mu} \boldsymbol{E} \\
\therefore \nabla &
\end{aligned}
$$$$
\text { (Replace } \mu_{r} \text { and } \varepsilon_{r} \text { ) }
$$$$
\text { (Common denominator) }
$$$$
\text { (Rearrange fractions, } c=\frac{1}{\varepsilon_{0} \mu_{0}} \text { ) }
$$$$
\text { (Multiply top and bottom by i) }
$$$$
\text { (Factor in i on the bottom) }
$$ 


$$
\begin{aligned}
& \frac{\partial \boldsymbol{H}}{\partial x}=-\mathrm{i} \frac{2 \pi N}{\lambda} \alpha \mathcal{H} \exp \left[\mathrm{i}\left(\omega t-\frac{2 \pi N}{\lambda}(\alpha x+\beta y+\gamma z)\right)\right] \\
& =-\mathrm{i} \frac{2 \pi N}{\lambda} \alpha \boldsymbol{H} \\
& =-\mathrm{i} \frac{\omega N}{c} \alpha \boldsymbol{H} \\
& \frac{\partial \boldsymbol{H}}{\partial y}=-\mathrm{i} \frac{\omega N}{c} \beta \boldsymbol{H} \\
& \frac{\partial \boldsymbol{H}}{\partial z}=-\mathrm{i} \frac{\omega N}{c} \gamma \boldsymbol{H} \\
& \nabla \times \boldsymbol{H}=\left(\frac{\partial \boldsymbol{H}}{\partial y}-\frac{\partial \boldsymbol{H}}{\partial z}\right) i+\left(\frac{\partial \boldsymbol{H}}{\partial z}-\frac{\partial \boldsymbol{H}}{\partial x}\right) j+\left(\frac{\partial \boldsymbol{H}}{\partial x}-\frac{\partial \boldsymbol{H}}{\partial y}\right) k \\
& \text { (Definition of the curl of } \boldsymbol{H} \text { ) } \\
& =-\mathrm{i} \frac{\omega N}{c}[(\beta \boldsymbol{H}-\gamma \boldsymbol{H}) i+(\gamma \boldsymbol{H}-\alpha \boldsymbol{H}) j+(\alpha \boldsymbol{H}-\beta \boldsymbol{H})] \\
& \text { (Substitute Eq. A.21, A.22, A.23) } \\
& =-\mathrm{i} \frac{\omega N}{c}(\hat{s} \times \boldsymbol{H}) \\
& -\mathrm{i} \frac{\omega N}{c}(\hat{s} \times \boldsymbol{H})=\mathrm{i} \frac{N^{2} \omega}{c^{2} \mu} \boldsymbol{E} \\
& (\hat{s} \times \boldsymbol{H})=-\frac{N}{c \mu} \boldsymbol{E} \\
& \therefore \boldsymbol{E}=-\frac{c \mu}{N}(\hat{s} \times \boldsymbol{H}) \\
& \therefore \boldsymbol{H}=\frac{N}{c \mu}(\hat{s} \times \boldsymbol{E})
\end{aligned}
$$

The optical admittance of free space is given by $\mathcal{Y}=\sqrt{\frac{\varepsilon_{0}}{\mu_{0}}}$. Typically, $\mu=\mu_{r} \mu_{0}$, but for optical frequencies $\mu_{r}=1$, so $\mu \approx \mu_{0}$. As such, the characteristic optical 
admittance of any medium can be given by the following expression:

$$
\begin{aligned}
\mathcal{y} & =\frac{N}{c \mu} \\
& =\frac{N \sqrt{\varepsilon_{0} \mu_{0}}}{\mu_{0}} \\
& =\frac{N \sqrt{\varepsilon_{0}} \sqrt{\mu_{0}}}{\left(\sqrt{\mu_{0}}\right)^{2}} \\
& =N \sqrt{\frac{\varepsilon_{0}}{\mu_{0}}} \\
\therefore \mathcal{Y} & =N \mathcal{Y}
\end{aligned}
$$

$$
\left(\mu \approx \mu_{0}, c=\frac{1}{\sqrt{\varepsilon_{0} \mu_{0}}}\right)
$$

(Rewrite the denominator)

That being the case, Eq. A.24 can be rewritten in the more convenient manner shown below.

$$
\begin{aligned}
& \boldsymbol{H}=\mathcal{Y}(\hat{s} \times \boldsymbol{E}) \\
& \boldsymbol{H}=N \mathcal{Y}(\hat{s} \times \boldsymbol{E})
\end{aligned}
$$

\section{A.1.4 The Poynting Vector, Irradiance, and the Optical Ab- sorption Coefficient}

In optics, the Poynting vector is defined as the instantaneous rate of flow of energy across unit area and is oriented along the same direction as $\hat{s}$. That being the case, the mean value of the Poynting vector is defined as the optical Irradiance (i.e. the optical intensity), $\boldsymbol{I}$, shown below where $\boldsymbol{H}^{*}$ is the complex conjugate of $\boldsymbol{H}$, whereas $E, H$, and $I$ are the respective scalar magnitudes.

$$
\begin{aligned}
\boldsymbol{I} & =\frac{1}{2} \mathbb{R}\left\{\boldsymbol{E} \times \boldsymbol{H}^{*}\right\} \\
\|\boldsymbol{I}\| & =I=\mathbb{R}\left\{\frac{1}{2} E H^{*}\right\}
\end{aligned}
$$


By incorporating in the expression derived for $\boldsymbol{H}$ in Eq. A.24, we can express the scalar irradiance as shown below.

$$
\begin{aligned}
\boldsymbol{I} & =\frac{1}{2} \mathbb{R}\{\boldsymbol{E} \times \mathcal{Y}(\hat{s} \times \boldsymbol{E})\} \\
I & =\mathbb{R}\left\{\frac{1}{2} \mathcal{Y} E E^{*} \hat{s}\right\} \\
\therefore I & =\frac{1}{2} n \mathcal{Y} E E^{*}
\end{aligned}
$$

In order to gain a more useful expression for the irradiance, however, we must first determine the Real component of the Electric vector amplitude.

$$
\begin{aligned}
\|\boldsymbol{E}\| & =E=\mathcal{E} \exp \left[\mathrm{i}\left(\omega t-\frac{2 \pi N}{\lambda}(\alpha x+\beta y+\gamma z)\right)\right] \quad \text { (From Eq. A.15) } \\
& =\mathcal{E} \exp \left[\mathrm{i}\left(\omega t-\frac{2 \pi[n-\mathrm{i} k]}{\lambda}(\alpha x+\beta y+\gamma z)\right)\right] \quad(N=\mathrm{i} k) \\
& =\mathcal{E} \exp \left[\frac{-2 \pi k}{\lambda}(\alpha x+\beta y+\gamma z)\right] \exp \left[\mathrm{i}\left(\omega t-\frac{2 \pi n}{\lambda}(\alpha x+\beta y+\gamma z)\right)\right] \\
\mathbb{R}\{E\} & =\mathcal{E} \exp \left[\frac{-2 \pi k}{\lambda}(\alpha x+\beta y+\gamma z)\right] \\
\therefore E E^{*} & =\mathcal{E E}^{*} \exp \left[\frac{-4 \pi k}{\lambda}(\alpha x+\beta y+\gamma z)\right]
\end{aligned}
$$

As a result, the Irradiance can be concisely represented in the following manner.

$$
I=\frac{1}{2} n \mathcal{Y}|\mathcal{E}|^{2} \exp \left[\frac{-4 \pi k}{\lambda}(\alpha x+\beta y+\gamma z)\right]
$$

Since the amplitude of the Electric field at $(x, y, z)$ is given by Eq. A.32, the expression for Irradiance can be generalized as $I=\frac{1}{2} n \mathcal{Y}$ (amplitude) ${ }^{2}$. Therefore,

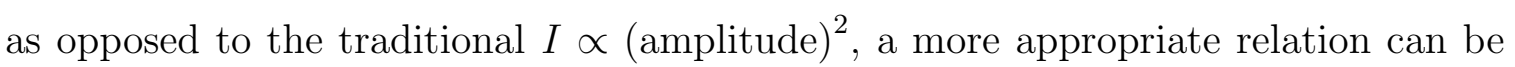


expressed as follows.

$$
I \propto n \times(\text { amplitude })^{2}
$$

Additionally, for expressions utilizing $\hat{s},(\alpha x+\beta y+\gamma z)$ is defined as the distance along the direction of propagation. Following from that expression, it can be found that $I$ drops to $\frac{1}{e} I$ at a distance given by $\frac{\lambda}{4 \pi k}$. It is convenient to define the optical absorption coefficient of a medium as the inverse of this distance:

$$
\alpha=\frac{4 \pi k}{\lambda}
$$

\section{A.1.5 Snell's Law}

We can readily derive Snell's law by applying a few simple boundary conditions, namely that:

- tangential components of $\boldsymbol{E}$ and $\boldsymbol{H}$ are continuous across the boundary

- $\hat{s}_{\text {reflected }}=\left\langle\alpha_{r}, \beta_{r}, \gamma_{r}\right\rangle$

- $\hat{s}_{\text {transmitted }}=\left\langle\alpha_{t}, \beta_{t}, \gamma_{t}\right\rangle$

From these conditions, we can represent each of the three wavefronts of interest - incident, reflected, and transmitted - consistently for both Electric and Magnetic components in the following forms:

$$
\begin{array}{r}
\text { Incident : } \exp \left[\mathrm{i}\left(\omega_{i} t-\left(\frac{2 \pi n_{0}}{\lambda_{i}}\right)\left(x \sin \vartheta_{0}+z \cos \vartheta_{0}\right)\right)\right] \\
\text { Reflected }: \exp \left[\mathrm{i}\left(\omega_{r} t-\left(\frac{2 \pi n_{0}}{\lambda_{r}}\right)\left(\alpha_{r} x+\beta_{r} y+\gamma_{r} z\right)\right)\right] \\
\text { Transmitted : } \exp \left[\mathrm{i}\left(\omega_{t} t-\left(\frac{2 \pi n_{1}}{\lambda_{t}}\right)\left(\alpha_{t} x+\beta_{t} y+\gamma_{t} z\right)\right)\right]
\end{array}
$$


Then, in order to satisfy continuity for all $x, y$, and $t$ at $z=0$, it can be determined that $\omega_{i} \equiv \omega_{r} \equiv \omega_{t}$. In other words, there is no change in frequency for reflected or transmitted waves as compared to the incident wave. That being the case, we can extend the identical equality to the wavelength, such that $\lambda_{i} \equiv \lambda_{r} \equiv \lambda_{t}$. Additionally, since the representation of the incident beam is oriented such that it is independent of $y$, the conclusion can be drawn that the directions of the reflected and transmitted waves are confined to the plane of incidence. The mathematical implications of these observations are as follows:

$$
\begin{aligned}
0 & \equiv n_{0} \beta_{r} \equiv n_{1} \beta_{t} \\
\left(\frac{2 \pi n_{0}}{\lambda}\right)\left(x \sin \vartheta_{i}+z \cos \vartheta_{i}\right) & \equiv\left(\frac{2 \pi n_{0}}{\lambda}\right)\left(\alpha_{r} x+\gamma_{r} z\right) \\
& \equiv\left(\frac{2 \pi n_{1}}{\lambda}\right)\left(\alpha_{t} x+\gamma_{t} z\right) \\
\therefore \sin \vartheta & =\alpha \\
\therefore \cos \vartheta & =\gamma \\
n_{0} \sin \vartheta_{i} & \equiv n_{0} \alpha_{r} \equiv n_{1} \alpha_{t}
\end{aligned}
$$

Following from Eq. A.37 and defining the angles of reflection and transmission to be $\vartheta_{r}$ and $\vartheta_{t}$ respectively, we find the following relation to be true.

$$
\begin{aligned}
n_{0} \sin \vartheta_{i} & \equiv n_{1} \alpha_{t} \\
& =n_{1} \sin \vartheta_{t}
\end{aligned}
$$

Redefining $\vartheta_{i}$ and $\vartheta_{t}$ to be $\vartheta_{0}$ and $\vartheta_{1}$ respectively, results in the traditional expression for Snell's Law shown below. Additionally, we can represent this expression 
equivalently as shown in Eq. A.40 and A.41.

$$
\begin{gathered}
n_{0} \sin \vartheta_{0}=n_{1} \sin \vartheta_{1} \\
\alpha_{r}^{2}+\gamma_{r}^{2}=1 \\
\alpha_{t}^{2}+\gamma_{t}^{2}=1
\end{gathered}
$$

\section{A.2 Boundary conditions}

This section deals with the various boundary conditions encountered when dealing with borders between two adjacent media. The first sections will deal with nonabsorbing media for simplicity, seeing as the absorbing nature of a medium necessitates the incorporation of complex components.

\section{A.2.1 Normal incidence in non-absorbing media}

This section deals with perhaps the simplest boundary conditions in that they do not incorporate angular components and deal only with straightforward interactions. The pair of conditions are that the electric and magnetic vectors are continuous across the boundary. They are represented mathematically as shown below:

$$
\begin{aligned}
\mathcal{E}_{i}+\mathcal{E}_{r} & =\mathcal{E}_{t} \\
\mathcal{H}_{i}-\mathcal{H}_{r} & =\mathcal{H}_{t}
\end{aligned}
$$

It is convenient to rearrange these conditions such that they deal with only the electric field; although the choice between $\mathcal{E}$ and $\mathcal{H}$ is an arbitrary one. Rewriting the second condition in terms of $\mathcal{E}$ results in the following derivations of the amplitude reflection coefficient, $\rho$, and the amplitude transmission coefficient, $\tau$. 


$$
\begin{aligned}
& \mathcal{H}_{i}-\mathcal{H}_{r}=\mathcal{H}_{t} \\
& { }_{\mathcal{Y}_{0}} \mathcal{E}_{i}-{ }_{\mathcal{Y}_{0}} \mathcal{E}_{r}={ }_{\mathcal{Y}_{1}} \mathcal{E}_{t} \\
& =\mathcal{Y}_{1}\left(\mathcal{E}_{i}+\mathcal{E}_{r}\right) \\
& \mathcal{E}_{i}\left(\mathcal{Y}_{0}-\mathcal{Y}_{1}\right)=\mathcal{E}_{r}\left(\mathcal{Y}_{0}+\mathcal{Y}_{1}\right) \\
& \frac{\mathcal{E}_{r}}{\mathcal{E}_{i}}=\frac{\mathcal{Y}_{0}-\mathcal{Y}_{1}}{\mathcal{Y}_{0}+\mathcal{Y}_{1}} \\
& =\frac{n_{0} \mathcal{Y}-n_{1} \mathcal{Y}}{n_{0} \mathcal{Y}+n_{1} \mathcal{Y}} \\
& =\frac{n_{0}-n_{1}}{n_{0}+n_{1}} \\
& \rho=\frac{\mathcal{E}_{r}}{\mathcal{E}_{i}} \\
& \therefore \rho=\frac{n_{0}-n_{1}}{n_{0}+n_{1}} \\
& { }_{\mathcal{Y}_{0}} \mathcal{E}_{i}-{ }_{\mathcal{Y}_{0}} \mathcal{E}_{r}={ }_{\mathcal{Y}_{1}} \mathcal{E}_{t} \\
& { }_{\mathcal{Y}_{0}} \mathcal{E}_{i}-\mathcal{Y}_{0}\left(\mathcal{E}_{t}-\mathcal{E}_{i}\right)={ }_{\mathcal{Y}_{1}} \mathcal{E}_{t} \\
& { }^{\mathcal{Y}_{0}} \mathcal{E}_{i}=\mathcal{E}_{t}\left(\mathcal{Y}_{0}+\mathcal{Y}_{1}\right) \\
& \frac{\mathcal{E}_{t}}{\mathcal{E}_{i}}=\frac{2 \mathcal{Y}_{0}}{\mathcal{Y}_{0}+\mathcal{Y}_{1}} \\
& =\frac{2 n_{0}}{n_{0}+n_{1}} \\
& \tau=\frac{\mathcal{E}_{t}}{\mathcal{E}_{i}} \\
& \therefore \tau=\frac{2 n_{0}}{n_{0}+n_{1}} \\
& (\mathcal{H}=\mathcal{Y} \mathcal{E}) \\
& (\mathcal{Y}=n \mathcal{Y})
\end{aligned}
$$

From these expressions for $\rho$ and $\tau$, we can observe that $\tau$ is always positive and real, indicating that there is no phase shift between incident and transmitted beams. We can also see that if $\rho$ is positive (i.e. if $n_{0}>n_{1}$ ), then there will be no phase change; however, if $\rho$ is negative, there will be a phase change of $\pi$. 
Having defined $\rho$ and $\tau$, we can determine expressions for the Irradiance of each of the three beams of interest - incident, reflected, and transmitted.

$$
\begin{aligned}
I & =\frac{1}{2} \mathcal{Y}_{\mathcal{E}} \mathcal{E}^{*} \\
\therefore I_{i} & =\frac{1}{2} \mathcal{Y}_{0} \mathcal{E}_{i} \mathcal{E}_{i}^{*}
\end{aligned}
$$

Here, $I_{i}$ above represents the Irradiance of the incident beam. While we can express $I_{r}$ and $I_{t}$ in similar terms, it is more convenient for future derivations to express them instead in terms of $I_{i}$, as shown below.

$$
\begin{aligned}
I_{r} & =\frac{1}{2} \mathcal{Y}_{0} \mathcal{E}_{r} \mathcal{E}_{r}^{*} \\
& =\frac{1}{2} \mathcal{Y}_{0}\left(\rho \mathcal{E}_{i}\right)\left(\rho \mathcal{E}_{i}\right)^{*} \\
& =\frac{1}{2} \mathcal{Y}_{0} \rho^{2} \mathcal{E}_{i} \mathcal{E}_{i}^{*} \\
\therefore I_{r} & =\rho^{2} I_{i} \\
I_{t} & =\frac{1}{2} \mathcal{Y}_{1} \mathcal{E}_{t} \mathcal{E}_{t}^{*} \\
& =\frac{1}{2} \mathcal{Y}_{1}\left(\tau \mathcal{E}_{i}\right)\left(\tau \mathcal{E}_{i}\right)^{*} \\
& =\frac{1}{2} \mathcal{y 0}_{0} \frac{\mathcal{y}_{1}}{\mathcal{y}_{0}} \tau^{2} \mathcal{E}_{i} \mathcal{E}_{i}^{*} \\
\therefore I_{t} & =\frac{\mathcal{y}_{1}}{\mathcal{y}_{0}} \tau^{2} I_{i}
\end{aligned}
$$$$
\text { (Substitute } \left.\mathcal{E}_{r}=\rho \mathcal{E}_{i}\right)
$$$$
\text { (Reflect the format of } I_{i} \text { ) }
$$

(Transmitted irradiance)

$$
\left(\text { Substitute } \mathcal{E}_{t}=\tau \mathcal{E}_{i}\right)
$$$$
\text { (Reflect the format of } I_{i} \text { ) }
$$

At this point, we can utilize our expressions for $\rho$ and $\tau$ along with the expressions for Irradiance shown above in order to derive the expressions for the relative reflection and transmission coefficients $R=\frac{I_{r}}{I_{i}}$ and $T=\frac{I_{t}}{I_{i}}$ through a simple energy balance equation based on Eq. A.28. 


$$
\begin{aligned}
& \boldsymbol{I}_{0}=\boldsymbol{I}_{1} \\
& \mathbb{R}\left\{\frac{1}{2} \boldsymbol{E}_{0} \times \boldsymbol{H}_{0}^{*}\right\}=\mathbb{R}\left\{\frac{1}{2} \boldsymbol{E}_{1} \times \boldsymbol{H}_{1}^{*}\right\} \\
& \mathbb{R}\left\{\frac{1}{2}\left(E_{i}+E_{r}\right)\left(H_{i}-H_{r}\right)^{*}\right\}=\mathbb{R}\left\{\frac{1}{2} E_{t} H_{t}^{*}\right\} \\
& \frac{1}{2}\left(\mathcal{E}_{i}+\mathcal{E}_{r}\right)\left(\mathcal{Y}_{0} \mathcal{E}_{i}-\mathcal{Y}_{0} \mathcal{E}_{r}\right)^{*}=\frac{1}{2} \mathcal{Y}_{1} \mathcal{E}_{t} \mathcal{E}_{t}^{*} \\
& \frac{1}{2} \mathcal{Y}_{0}\left(\mathcal{E}_{i}+\rho \mathcal{E}_{i}\right)\left(\mathcal{E}_{i}-\rho \mathcal{E}_{i}\right)^{*}=\frac{1}{2} \mathcal{Y}_{0} \frac{\mathcal{Y}_{1}}{\mathcal{Y}_{0}} \tau^{2} \mathcal{E}_{i} \mathcal{E}_{i}^{*} \\
& \frac{1}{2} \mathcal{Y}_{0} \mathcal{E}_{i} \mathcal{E}_{i}^{*}\left(1-\rho^{2}\right)=\frac{1}{2} \mathcal{Y}_{0} \frac{\mathcal{Y}_{1}}{\mathcal{Y}_{0}} \tau^{2} \mathcal{E}_{i} \mathcal{E}_{i}^{*} \\
& I_{i}\left(1-\rho^{2}\right)=\frac{\mathcal{Y}_{1}}{\mathcal{Y}_{0}} \tau^{2} I_{i} \\
& I_{i}-\rho^{2} I_{i}=\frac{\mathcal{Y} 1}{\mathcal{Y}_{0}} \tau^{2} I_{i} \\
& I_{i}-I_{r}=I_{t} \\
& 1-\frac{I_{r}}{I_{i}}=\frac{I_{t}}{I_{i}} \\
& \therefore 1-R=T \\
& (\mathcal{H}=\mathcal{Y} \mathcal{E}) \\
& \left(\mathcal{E}_{r}=\rho \mathcal{E}_{i}, \mathcal{E}_{t}=\tau \mathcal{E}_{i}\right)
\end{aligned}
$$

\section{A.2.2 Oblique incidence in non-absorbing media}

\section{A.2.2.1 p-polarized light (TM polarized)}

This section deals with oblique incidence, and therefore necessitates separate approaches based on the polarization state of the incident beam - either p- or s- polarized. The two approaches are similar, but require different sign conventions and result in slightly differing representations for the relative reflection and transmission coefficients. We will begin by stating the boundary conditions for p-polarized light, 
mathematically represented as shown below:

$$
\begin{aligned}
\mathcal{E}_{i} \cos \vartheta_{0}+\mathcal{E}_{r} \cos \vartheta_{0} & =\mathcal{E}_{t} \cos \vartheta_{1} \\
\mathcal{H}_{i}-\mathcal{H}_{r} & =\mathcal{H}_{t} \\
\mathcal{y}_{0} \mathcal{E}_{i}-\mathcal{y}_{0} \mathcal{E}_{r} & =\mathcal{Y}_{1} \mathcal{E}_{t}
\end{aligned}
$$

Again, we will rearrange these conditions such that they deal with only the electric field. It is important to note that the $\cos \vartheta$ terms arise from translating $\hat{s}$ over to perpendicular incidence, so that $\boldsymbol{E}$ and $\boldsymbol{H}$ are tangential (parallel) to the surface. Furthermore, it is convenient to define new variables to represent components of the translated boundary conditions, as shown below:

$$
\begin{aligned}
\mathrm{E}_{i} & =\mathcal{E}_{i} \cos \vartheta_{0} & \mathrm{H}_{i} & =\mathcal{y}_{i} \mathcal{E}_{i}=\frac{\mathcal{y}_{i}}{\cos \vartheta_{0}} \mathrm{E}_{i} \\
\mathrm{E}_{r} & =\mathcal{E}_{r} \cos \vartheta_{0} & \mathrm{H}_{r} & =\frac{\mathcal{y}_{r}}{\cos \vartheta_{0}} \mathrm{E}_{r} \\
\mathrm{E}_{t} & =\mathcal{E}_{t} \cos \vartheta_{1} & \mathrm{H}_{t} & =\frac{\mathcal{y}_{t}}{\cos \vartheta_{1}} \mathrm{E}_{t}
\end{aligned}
$$

Rewriting the boundary conditions in the terms shown above yields Eq. A.52 and A.53, from which we can obtain expressions for the amplitude reflection and transmission coefficients for p-polarized light, $\rho_{p}$ and $\tau_{p}$ respectively. 


$$
\begin{aligned}
& \mathrm{E}_{i}+\mathrm{E}_{r}=\mathrm{E}_{t} \\
& \frac{\mathcal{Y}_{0}}{\cos \vartheta_{0}} \mathrm{E}_{i}-\frac{\mathcal{Y}_{0}}{\cos \vartheta_{0}} \mathrm{E}_{r}=\frac{\mathcal{Y}_{1}}{\cos \vartheta_{1}} \mathrm{E}_{t} \\
& =\frac{\mathcal{y}_{1}}{\cos \vartheta_{1}}\left(\mathrm{E}_{i}+\mathrm{E}_{r}\right) \\
& \mathrm{E}_{i}\left(\frac{\mathcal{Y}_{0}}{\cos \vartheta_{0}}-\frac{\mathcal{y}_{1}}{\cos \vartheta_{1}}\right)=\mathrm{E}_{r}\left(\frac{\mathcal{Y}_{0}}{\cos \vartheta_{0}}+\frac{\mathcal{y}_{1}}{\cos \vartheta_{1}}\right) \\
& \frac{\mathrm{E}_{r}}{\mathrm{E}_{i}}=\frac{\frac{\mathcal{y}_{0}}{\cos \vartheta_{0}}-\frac{\mathcal{y} 1}{\cos \vartheta_{1}}}{\frac{\mathcal{y} 0}{\cos \vartheta_{0}}+\frac{\mathcal{Y} 1}{\cos \vartheta_{1}}} \\
& \rho_{p}=\frac{\mathrm{E}_{r}}{\mathrm{E}_{i}} \\
& \therefore \rho_{p}=\frac{\frac{y_{0}}{\cos \vartheta_{0}}-\frac{y_{1}}{\cos \vartheta_{1}}}{\frac{y^{0}}{\cos \vartheta_{0}}+\frac{y_{1}}{\cos \vartheta_{1}}} \\
& \frac{\mathcal{Y}_{0}}{\cos \vartheta_{0}} \mathrm{E}_{i}-\frac{\mathcal{Y}_{0}}{\cos \vartheta_{0}} \mathrm{E}_{r}=\frac{\mathcal{Y}_{1}}{\cos \vartheta_{1}} \mathrm{E}_{t} \\
& \frac{\mathcal{Y}_{0}}{\cos \vartheta_{0}} \mathrm{E}_{i}-\frac{\mathcal{Y}_{0}}{\cos \vartheta_{0}}\left(\mathrm{E}_{t}-\mathrm{E}_{i}\right)=\frac{\mathcal{Y}_{1}}{\cos \vartheta_{1}} \mathrm{E}_{t} \\
& \frac{2 \mathcal{Y}_{0}}{\cos \vartheta_{0}} \mathrm{E}_{i}=\mathrm{E}_{t}\left(\frac{\mathcal{Y}_{0}}{\cos \vartheta_{0}}+\frac{\mathcal{Y}_{1}}{\cos \vartheta_{1}}\right) \\
& \frac{\mathrm{E}_{t}}{\mathrm{E}_{i}}=\frac{\frac{2 \mathcal{y}_{0}}{\cos \vartheta_{0}}}{\frac{\mathcal{y} 0}{\cos \vartheta_{0}}+\frac{\mathcal{Y} 1}{\cos \vartheta_{1}}} \\
& \tau_{p}=\frac{\mathrm{E}_{t}}{\mathrm{E}_{i}} \\
& \therefore \tau_{p}=\frac{\frac{2 y_{0}}{\cos \vartheta_{0}}}{\frac{\mathcal{y} 0}{\cos \vartheta_{0}}+\frac{\mathcal{Y} 1}{\cos \vartheta_{1}}}
\end{aligned}
$$

(Substitute in Eq. A.52)

(Distribute and factor)

(Definition of $\rho$ )

(Continued from above)

From here we define the expressions for the Irradiance of each of the three beams of interest. Considering that the oblique incidence will add some otherwise unexpected components to the Irradiance, we will begin with the original definition 
for the optical Irradiance, $\boldsymbol{I}$, and proceed to find expressions for $I_{i}, I_{r}$, and $I_{t}$.

$$
\begin{aligned}
\boldsymbol{I} & =\frac{1}{2} \mathbb{R}\left\{\boldsymbol{E} \times \boldsymbol{H}^{*}\right\} \\
I & =\mathbb{R}\left\{\frac{1}{2} \mathrm{E}\left(\frac{\mathcal{y}}{\cos \vartheta} \mathrm{E}\right)^{*}\right\} \\
\therefore I_{i} & =\frac{1}{2}\left(\frac{\mathcal{y}_{0}}{\cos \vartheta_{0}}\right) \mathrm{E}_{i} \mathrm{E}_{i}^{*}
\end{aligned}
$$

Again, $I_{i}$ represents the Irradiance of the incident beam. We will also express $I_{r}$ and $I_{t}$ in terms of $I_{i}$ as we did in the previous section in order to derive expressions for the relative reflection and transmission coefficients for p-polarized light, $R_{p}$ and $T_{p}$ respectively.

$$
\begin{aligned}
& I_{r}=\mathbb{R}\left\{\frac{1}{2} \mathrm{E}_{r}\left(\frac{\mathcal{y}_{0}}{\cos \vartheta_{0}} \mathrm{E}_{r}\right)^{*}\right\} \\
& =\frac{1}{2} \rho_{p} \mathrm{E}_{i}\left(\frac{\mathcal{y}_{0}}{\cos \vartheta_{0}} \rho_{p} \mathrm{E}_{i}\right)^{*} \\
& =\rho_{p}^{2} \frac{1}{2}\left(\frac{\mathcal{Y}_{0}}{\cos \vartheta_{0}}\right) \mathrm{E}_{i} \mathrm{E}_{i}^{*} \\
& \therefore I_{r}=\rho^{2} I_{i} \\
& I_{t}=\mathbb{R}\left\{\frac{1}{2} \mathrm{E}_{t}\left(\frac{\mathcal{Y}_{1}}{\cos \vartheta_{1}} \mathrm{E}_{t}\right)^{*}\right\} \\
& =\frac{1}{2} \tau_{p} \mathrm{E}_{i}\left(\frac{\mathcal{y}_{1}}{\cos \vartheta_{1}} \tau_{p} \mathrm{E}_{i}\right)^{*} \\
& =\tau_{p}^{2} \frac{1}{2} \frac{\mathcal{y}_{1} \cos \vartheta_{0}}{\mathcal{y}_{0} \cos \vartheta_{1}}\left(\frac{\mathcal{y}_{0}}{\cos \vartheta_{0}}\right) \mathrm{E}_{i} \mathrm{E}_{i}^{*} \\
& \therefore I_{t}=\tau_{p}^{2} \frac{\mathcal{y}_{1} \cos \vartheta_{0}}{\mathcal{y}_{0} \cos \vartheta_{1}} I_{i} \\
& \left(\mathrm{E}_{r}=\rho_{p} \mathrm{E}_{i}\right) \\
& \text { (Reflect the format of } I_{i} \text { ) }
\end{aligned}
$$

Consider the simple energy balance equation below in order to derive $R_{p}$ and $T_{p}$. It is important to note that by translating $\hat{s}$ to perpendicular incidence by incorporating in the $\cos \vartheta \operatorname{components,~the~traditional~condition~that~} 1-R=T$ still holds 
true.

$$
\begin{aligned}
& \boldsymbol{I}_{0}=\boldsymbol{I}_{1} \\
& \mathbb{R}\left\{\frac{1}{2} \boldsymbol{E}_{0} \times \boldsymbol{H}_{0}^{*}\right\}=\mathbb{R}\left\{\frac{1}{2} \boldsymbol{E}_{1} \times \boldsymbol{H}_{1}^{*}\right\} \\
& \mathbb{R}\left\{\frac{1}{2}\left(\mathrm{E}_{i}+\mathrm{E}_{r}\right)\left(\mathrm{H}_{i}-\mathrm{H}_{r}\right)^{*}\right\}=\mathbb{R}\left\{\frac{1}{2} \mathrm{E}_{t} \mathrm{H}_{t}^{*}\right\} \\
& \frac{1}{2}\left(\mathrm{E}_{i}+\mathrm{E}_{r}\right)\left(\frac{\mathcal{y}_{0}}{\cos \vartheta_{0}} \mathrm{E}_{i}-\frac{\mathcal{Y}_{0}}{\cos \vartheta_{0}} \mathrm{E}_{r}\right)^{*}=\frac{1}{2} \mathrm{E}_{t} \frac{\mathcal{y}_{1}}{\cos \vartheta_{1}} \mathrm{E}_{t}^{*} \\
& \frac{1}{2} \frac{\mathcal{Y}_{0}}{\cos \vartheta_{0}}\left(\mathrm{E}_{i}+\rho_{p} \mathrm{E}_{i}\right)\left(\mathrm{E}_{i}-\rho_{p} \mathrm{E}_{i}\right)^{*}=\frac{1}{2} \frac{\mathcal{y}_{1}}{\cos \vartheta_{1}} \tau_{p}^{2} \mathrm{E}_{i} \mathrm{E}_{i}^{*} \\
& \frac{1}{2} \frac{\mathcal{y}_{0}}{\cos \vartheta_{0}} \mathrm{E}_{i} \mathrm{E}_{i}^{*}\left(1-\rho_{p}^{2}\right)=\frac{1}{2} \frac{\mathcal{y}_{1} \cos \vartheta_{0}}{\mathcal{y}_{0} \cos \vartheta_{1}} \frac{\mathcal{y}_{0}}{\cos \vartheta_{0}} \mathrm{E}_{i} \mathrm{E}_{i}^{*} \tau_{p}^{2} \\
& I_{i}\left(1-\rho_{p}^{2}\right)=\frac{\mathcal{y}_{1} \cos \vartheta_{0}}{\mathcal{y}_{0} \cos \vartheta_{1}} \tau_{p}^{2} I_{i} \\
& I_{i}-\rho_{p}^{2} I_{i}=\frac{\mathcal{y}_{1} \cos \vartheta_{0}}{\mathcal{y}_{0} \cos \vartheta_{1}} \tau_{p}^{2} I_{i} \\
& I_{i}-I_{r}=I_{t} \\
& 1-\frac{I_{r}}{I_{i}}=\frac{I_{t}}{I_{i}} \\
& \therefore 1-R_{p}=T_{p} \\
& \left(\mathrm{H}=\frac{y}{\cos \vartheta} \mathrm{E}\right)
\end{aligned}
$$

It may also be useful to express $R_{p}$ and $T_{p}$ in terms of $\mathcal{y}$ and $\vartheta$.

$$
\begin{aligned}
& R_{p}=\frac{I_{r}}{I_{i}} \\
& =\frac{\rho_{p}^{2} I_{i}}{I_{i}} \\
& =\rho_{p}^{2} \\
& \therefore R_{p}=\left[\frac{\frac{y_{0}}{\cos \vartheta_{0}}-\frac{\mathcal{y} 1}{\cos \vartheta_{1}}}{\frac{\mathcal{y} 0}{\cos \vartheta_{0}}+\frac{\mathcal{y} 1}{\cos \vartheta_{1}}}\right]^{2}
\end{aligned}
$$




$$
\begin{aligned}
T_{p} & =\frac{I_{t}}{I_{i}} \\
& =\frac{\frac{y_{1} \cos \vartheta_{0}}{y_{0} \cos \vartheta_{p}^{2} I_{i}}}{I_{i}} \\
& =\frac{\mathcal{y}_{1} \cos \vartheta_{0}}{\mathcal{y}_{0} \cos \vartheta_{1}} \tau_{p}^{2} \\
& =\frac{\mathcal{y}_{1} \cos \vartheta_{0}}{\mathcal{y}_{0} \cos \vartheta_{1}}\left[\frac{\frac{4 \mathcal{y}_{0}^{2}}{\cos \vartheta_{0}}}{\left(\frac{y_{0}}{\cos \vartheta_{0}}+\frac{\mathcal{y}_{1}}{\cos \vartheta_{1}}\right)^{2}}\right] \\
\therefore T_{p} & =\frac{\frac{4_{\mathcal{y}} \mathcal{y}_{1}}{\cos \vartheta_{0} \cos \vartheta_{1}}}{\left(\frac{y_{0}}{\cos \vartheta_{0}}+\frac{y_{1}}{\cos \vartheta_{1}}\right)^{2}}
\end{aligned}
$$

(Definition of $T_{p}$ )

\section{A.2.2.2 s-polarized light (TE polarized)}

Having derived the expressions for p-polarized light, we must now consider the case of s-polarized light. The boundary conditions for the s-polarization state are as follows:

$$
\begin{aligned}
\mathcal{E}_{i}+\mathcal{E}_{r} & =\mathcal{E}_{t} \\
\mathcal{H}_{i} \cos \vartheta_{0}-\mathcal{H}_{r} \cos \vartheta_{0} & =\mathcal{H}_{t} \cos \vartheta_{1}
\end{aligned}
$$

Again, we will rearrange the conditions such that they deal only with the electric field. We will also define new variables to represent the components of the translated boundary conditions, as we did for the p-polarization state.

$$
\begin{array}{ll}
\mathrm{E}_{i}=\mathcal{E}_{i} & \mathrm{H}_{i}=\mathcal{H}_{i} \cos \vartheta_{0}=\mathcal{y}_{0} \cos \vartheta_{0} \mathrm{E}_{i} \\
\mathrm{E}_{r}=\mathcal{E}_{r} & \mathrm{H}_{r}=\mathcal{y}_{0} \cos \vartheta_{0} \mathrm{E}_{r} \\
\mathrm{E}_{t}=\mathcal{E}_{t} & \mathrm{H}_{t}=\mathcal{y}_{1} \cos \vartheta_{1} \mathrm{E}_{t}
\end{array}
$$

From there the derivation follows the same format as that of the p-polarized light. First we will obtain the expressions for the amplitude reflection and transmis- 
sion coefficients for s-polarized light, $\rho_{s}$ and $\tau_{s}$ respectively.

$$
\begin{aligned}
& \mathrm{H}_{i}-\mathrm{H}_{r}=\mathrm{H}_{t} \\
& y_{0} \cos \vartheta_{0} \mathrm{E}_{i}-y_{0} \cos \vartheta_{0} \mathrm{E}_{r}=y_{1} \cos \vartheta_{1} \mathrm{E}_{t} \\
& =y_{1} \cos \vartheta_{1}\left(\mathrm{E}_{i}+\mathrm{E}_{r}\right) \\
& \mathrm{E}_{i}\left(y_{0} \cos \vartheta_{0}-y_{1} \cos \vartheta_{1}\right)=\mathrm{E}_{r}\left(\mathcal{y}_{0} \cos \vartheta_{0}+\mathcal{y}_{1} \cos \vartheta_{1}\right) \\
& \frac{\mathrm{E}_{r}}{\mathrm{E}_{i}}=\frac{\mathcal{y}_{0} \cos \vartheta_{0}-\mathcal{y}_{1} \cos \vartheta_{1}}{\mathcal{y} \cos \vartheta_{0}+\mathcal{y} \cos \vartheta_{1}} \\
& \rho_{s}=\frac{\mathrm{E}_{r}}{\mathrm{E}_{i}} \\
& \therefore \rho_{s}=\frac{\mathcal{y}_{0} \cos \vartheta_{0}-\mathcal{y}_{1} \cos \vartheta_{1}}{\mathcal{y} \cos \vartheta_{0}+\mathcal{y} \cos \vartheta_{1}} \\
& y_{0} \cos \vartheta_{0} \mathrm{E}_{i}-y_{0} \cos \vartheta_{0} \mathrm{E}_{r}=y_{1} \cos \vartheta_{1} \mathrm{E}_{t} \\
& \mathcal{y}_{0} \cos \vartheta_{0} \mathrm{E}_{i}-\mathcal{y}_{0} \cos \vartheta_{0}\left(\mathrm{E}_{t}-\mathrm{E}_{i}\right)=\mathcal{y}_{1} \cos \vartheta_{1} \mathrm{E}_{t} \\
& \mathrm{E}_{i}\left(2 \mathcal{y}_{0} \cos \vartheta_{0}\right)=\mathrm{E}_{t}\left(\mathcal{y}_{0} \cos \vartheta_{0}+\mathcal{y}_{1} \cos \vartheta_{1}\right) \\
& \frac{\mathrm{E}_{t}}{\mathrm{E}_{i}}=\frac{2{\mathcal{y _ { 0 }}}_{0} \cos \vartheta_{0}}{\mathcal{y}_{0} \cos \vartheta_{0}+\mathcal{y}_{1} \cos \vartheta_{1}} \\
& \tau_{s}=\frac{\mathrm{E}_{t}}{\mathrm{E}_{i}} \\
& \therefore \tau_{s}=\frac{2 y_{0} \cos \vartheta_{0}}{y_{0} \cos \vartheta_{0}+y_{1} \cos \vartheta_{1}}
\end{aligned}
$$

Next we define the expressions for the Irradiance of each of the three beams of interest in a similar manner as before. Again, $I_{i}$ represents the Irradiance of the incident beam derived from the general expression for Irradiance, as shown below.

$$
\begin{aligned}
\boldsymbol{I} & =\frac{1}{2} \mathbb{R}\left\{\boldsymbol{E} \times \boldsymbol{H}^{*}\right\} \\
I & =\mathbb{R}\left\{\frac{1}{2} \mathrm{E}(y \cos \vartheta \mathrm{E})^{*}\right\} \\
\therefore I_{i} & =\frac{1}{2} y_{0} \cos \vartheta_{0} \mathrm{E}_{i} \mathrm{E}_{i}^{*}
\end{aligned}
$$


Following along the same approach as before, we will now define $I_{r}$ and $I_{t}$ in terms of $I_{i}$ in order to derive the relative reflection and transmission coefficients for s-polarized light, $R_{s}$ and $T_{s}$ respectively.

$$
\begin{aligned}
& I_{r}=\mathbb{R}\left\{\frac{1}{2} \mathrm{E}_{r}\left(\mathcal{y}_{0} \cos \vartheta_{0} \mathrm{E}_{r}\right)^{*}\right\} \\
& =\frac{1}{2} \rho_{s} \mathrm{E}_{i}\left(\mathcal{y}_{0} \cos \vartheta_{0} \rho_{s} \mathrm{E}_{i}\right)^{*} \\
& =\frac{1}{2} \rho_{s}^{2} \mathcal{Y}_{0} \cos \vartheta_{0} \mathrm{E}_{i} \mathrm{E}_{i}^{*} \\
& \therefore I_{r}=\rho_{s}^{2} I_{i} \\
& I_{t}=\mathbb{R}\left\{\frac{1}{2} \mathrm{E}_{t}\left(\mathcal{y}_{1} \cos \vartheta_{1} \mathrm{E}_{t}\right)^{*}\right\} \\
& =\frac{1}{2} \tau_{s} \mathrm{E}_{i}\left(\mathcal{y}_{1} \cos \vartheta_{1} \tau_{s} \mathrm{E}_{i}\right)^{*} \\
& =\frac{1}{2} \tau_{s}^{2} \frac{\mathcal{y}_{1} \cos \vartheta_{1}}{\mathcal{y}_{0} \cos \vartheta_{0}} \mathcal{y}_{0} \cos \vartheta_{0} \mathrm{E}_{i} \mathrm{E}_{i}^{*} \\
& \therefore I_{t}=\frac{\mathcal{y}_{1} \cos \vartheta_{1}}{\mathcal{y}_{0} \cos \vartheta_{0}} \tau_{s}^{2} I_{i} \\
& \left(\mathrm{E}_{r}=\rho_{s} \mathrm{E}_{i}\right) \\
& \left(\mathrm{E}_{t}=\tau_{s} \mathrm{E}_{i}\right)
\end{aligned}
$$

Consider the energy balance equation below in order to derive $R_{s}$ and $T_{s}$. As for the p-polarized condition, we will find that the traditional condition that $1-R=T$ still holds true. 


$$
\begin{aligned}
& \boldsymbol{I}_{0}=\boldsymbol{I}_{1} \quad \text { (Conservation of energy) } \\
& \mathbb{R}\left\{\frac{1}{2} \boldsymbol{E}_{0} \times \boldsymbol{H}_{0}^{*}\right\}=\mathbb{R}\left\{\frac{1}{2} \boldsymbol{E}_{1} \times \boldsymbol{H}_{1}^{*}\right\} \quad \text { (Sub in. Eq. A.28) } \\
& \mathbb{R}\left\{\frac{1}{2}\left(\mathrm{E}_{i}+\mathrm{E}_{r}\right)\left(\mathrm{H}_{i}-\mathrm{H}_{r}\right)^{*}\right\}=\mathbb{R}\left\{\frac{1}{2} \mathrm{E}_{t} \mathrm{H}_{t}^{*}\right\} \quad \text { (Scalar components) } \\
& \frac{1}{2}\left(\mathrm{E}_{i}+\mathrm{E}_{r}\right)\left(\mathcal{y}_{0} \cos \vartheta_{0} \mathrm{E}_{i}-\mathcal{y}_{0} \cos \vartheta_{0} \mathrm{E}_{r}\right)^{*}=\frac{1}{2} \mathrm{E}_{t \mathcal{y}_{1}} \cos \vartheta_{1} \mathrm{E}_{t}^{*} \quad(\mathrm{H}=\mathcal{y} \cos \vartheta \mathrm{E}) \\
& \frac{1}{2} \mathcal{Y}_{0} \cos \vartheta_{0}\left(\mathrm{E}_{i}+\rho_{s} \mathrm{E}_{i}\right)\left(\mathrm{E}_{i}-\rho_{s} \mathrm{E}_{i}\right)^{*}=\frac{1}{2} \mathcal{Y}_{1} \cos \vartheta_{1} \tau_{s}^{2} \mathrm{E}_{i} \mathrm{E}_{i}^{*} \quad\left(\mathrm{E}_{r}=\rho_{s} \mathrm{E}_{i}\right) \\
& \frac{1}{2} \mathcal{Y}_{0} \cos \vartheta_{0} \mathrm{E}_{i} \mathrm{E}_{i}^{*}\left(1-\rho_{s}^{2}\right)=\frac{1}{2} \mathcal{Y}_{1} \cos \vartheta_{1} \tau_{s}^{2} \mathrm{E}_{i} \mathrm{E}_{i}^{*} \quad \text { (Factor) } \\
& =\frac{1}{2} \frac{\mathcal{y}_{1} \cos \vartheta_{1}}{\mathcal{y}_{0} \cos \vartheta_{0}} \mathcal{Y}_{0} \cos \vartheta_{0} \tau_{s}^{2} \mathrm{E}_{i} \mathrm{E}_{i}^{*} \\
& I_{i}\left(1-\rho_{s}^{2}\right)=\frac{1}{2} \frac{\mathcal{y}_{1} \cos \vartheta_{1}}{\mathcal{Y}_{0} \cos \vartheta_{0}} I_{i} \\
& I_{i}-\rho_{s}^{2} I_{i}=\frac{1}{2} \frac{\mathcal{Y}_{1} \cos \vartheta_{1}}{\mathcal{Y}_{0} \cos \vartheta_{0}} I_{i} \\
& I_{i}-I_{r}=I_{t} \\
& 1-\frac{I_{r}}{I_{i}}=\frac{I_{t}}{I_{i}} \\
& \therefore 1-R=T
\end{aligned}
$$

Again, we will express $R_{s}$ and $T_{s}$ in terms of $\mathcal{y}$ and $\vartheta$.

$$
\begin{aligned}
R_{s} & =\frac{I_{r}}{I_{i}} \\
& =\frac{\rho_{s}^{2} I_{i}}{I_{i}} \\
& =\rho_{s}^{2} \\
\therefore R_{s} & =\left(\frac{\mathcal{y}_{0} \cos \vartheta_{0}-\mathcal{y}_{1} \cos \vartheta_{1}}{\mathcal{y}_{0} \cos \vartheta_{0}+\mathcal{y}_{1} \cos \vartheta_{1}}\right)^{2}
\end{aligned}
$$




$$
\begin{aligned}
T_{s} & =\frac{I_{t}}{I_{i}} \\
& =\frac{\tau_{s}^{2} \frac{\mathcal{y}_{1} \cos \vartheta_{1}}{\mathcal{y}_{0} \cos \vartheta_{0}} I_{i}}{I_{i}} \\
& =\tau_{s}^{2} \frac{\mathcal{Y}_{1} \cos \vartheta_{1}}{\mathcal{Y}_{0} \cos \vartheta_{0}} \\
& =\frac{\mathcal{Y}_{1} \cos \vartheta_{1}}{\mathcal{Y}_{0} \cos \vartheta_{0}} \frac{4 \mathcal{Y}_{0}^{2} \cos ^{2} \vartheta_{0}}{\left(\mathcal{y}_{0} \cos \vartheta_{0}+\mathcal{y}_{1} \cos \vartheta_{1}\right)^{2}} \\
\therefore T_{s} & =\frac{4 \mathcal{Y}_{0} \mathcal{Y}_{1} \cos \vartheta_{0} \cos \vartheta_{1}}{\left(\mathcal{y}_{0} \cos \vartheta_{0}+\mathcal{y}_{1} \cos \vartheta_{1}\right)^{2}}
\end{aligned}
$$

(Definition of $T_{s}$ )

\section{A.2.2.3 Optical admittance for oblique incidence}

The optical admittance for light incident upon a surface at an oblique angle takes a slightly different form than it did for normal incidence. Since the expressions for $\mathrm{H}$ and $\mathrm{E}$ differ based on the polarization state of the beam, we must redefine the optical admittance term in such a way that it applies for any obliquely incident beam upon a non-absorbing medium, independent of its polarization. In order to distinguish this term from the optical admittance for normal incidence, we will define it as follows:

$$
\begin{aligned}
& \text { normal incidence }: \eta=\mathcal{Y}=n \mathcal{Y} \\
& \text { oblique incidence }: \eta=\frac{\mathrm{H}}{\mathrm{E}}
\end{aligned}
$$

Proceeding from this definition for oblique incidence, we can derive new expressions for $\rho$ and $\tau$ that are applicable regardless of the polarization state of the incident beam. In order to demonstrate this, we will derive the expression in sequence for pand s- polarizations. 


$$
\begin{aligned}
\eta_{p} & =\frac{\mathcal{Y}}{\cos \vartheta} & \left(\frac{\mathrm{H}}{\mathrm{E}} \text { for p-polarized light }\right) \\
\rho_{p} & =\frac{\frac{\mathcal{y} 0}{\cos \vartheta_{0}}-\frac{\mathcal{y} 1}{\cos \vartheta_{1}}}{\frac{\mathcal{y} 0}{\cos \vartheta_{0}}+\frac{\mathcal{Y} 1}{\cos \vartheta_{1}}} & \text { (Definition of } \left.\rho_{p}\right) \\
& =\frac{\eta_{p, 0}-\eta_{p, 1}}{\eta_{p, 0}+\eta_{p, 1}} & \text { (Substitute in } \left.\eta_{p}\right)
\end{aligned}
$$

$$
\begin{aligned}
\eta_{s} & =y_{\operatorname{y}} \cos \vartheta \\
\rho_{s} & =\frac{\mathcal{y}_{0} \cos \vartheta_{0}-\mathcal{y}_{1} \cos \vartheta_{1}}{\mathcal{y}_{0} \cos \vartheta_{0}+\mathcal{y}_{1} \cos \vartheta_{1}} \\
& =\frac{\eta_{s, 0}-\eta_{s, 1}}{\eta_{s, 0}+\eta_{s, 1}}
\end{aligned}
$$

The two derivations reveal that the amplitude reflection coefficient, $\rho$, can be represented in the same manner irrespective of the polarization state of light by writing it in terms of the optical admittance, $\eta$.

$$
\therefore \rho=\frac{\eta_{0}-\eta_{1}}{\eta_{0}+\eta_{1}}
$$

Continuing in a similar manner, we can derive an expression for the amplitude transmission coefficient, $\tau$. The resultant equation is again independent of the polarization state, provided the appropriate optical admittance is used.

$$
\begin{aligned}
\eta_{p} & =\frac{\mathcal{Y}}{\cos \vartheta} & \left(\frac{\mathrm{H}}{\mathrm{E}} \text { for p-polarized light }\right) \\
\tau_{p} & =\frac{\frac{2 \mathcal{y}_{0}}{\cos \vartheta_{0}}}{\frac{\mathcal{Y} 0}{\cos \vartheta_{0}}+\frac{\mathcal{Y} 1}{\cos \vartheta_{1}}} & \left(\text { Definition of } \tau_{p}\right) \\
& =\frac{2 \eta_{p, 0}}{\eta_{p, 0}+\eta_{p, 1}} & \left(\text { Substitute in } \eta_{p}\right)
\end{aligned}
$$




$$
\begin{array}{rlr}
\eta_{s}= & \mathcal{y} \cos \vartheta & \left(\frac{\mathrm{H}}{\mathrm{E}}\right. \text { for s-polarized light) } \\
\tau_{s}=\frac{2 \mathcal{Y}_{0} \cos \vartheta_{0}}{\mathcal{Y}_{0} \cos \vartheta_{0}+\mathcal{y}_{1} \cos \vartheta_{1}} & \text { (Definition of } \left.\tau_{s}\right) \\
=\frac{2 \eta_{s, 0}}{\eta_{s, 0}+\eta_{s, 1}} & \text { (Substitute in } \left.\eta_{s}\right) \\
& \therefore \tau=\frac{2 \eta_{0}}{\eta_{0}+\eta_{1}}
\end{array}
$$

Based on these new definitions, we can further express the relative reflection and transmission coefficients, $R$ and $T$ in terms of the optical admittance, $\eta$, as shown below.

$$
\begin{aligned}
& R=\left(\frac{\eta_{0}-\eta_{1}}{\eta_{0}+\eta_{1}}\right)^{2} \\
& T=\frac{4 \eta_{0} \eta_{1}}{\left(\eta_{0}+\eta_{1}\right)^{2}}
\end{aligned}
$$

\section{A.2.2.4 The Brewster Angle}

The equations derived above can be used to determine the degree of reflectance for each polarization at a simple boundary between extended media. In cases wherein there is no absorption in the medium, it can be determined that there is a definitive angle at which the reflectance for p-polarized light goes to zero. This angle is referred to as the Brewster Angle, but is also known as the polarizing angle, since the all of the reflected light will be s-polarized. The expression for this angle can be derived using a combination of Snell's Law (Eq. A.39) and the expression for the relative reflection coefficient for p-polarized light (Eq. A.60). Since the Brewster angle is defined as the angle at which $R_{p}=0$, we will start with the conditions necessary for that outcome to occur, and derive an expression that allows us to eliminate $\vartheta_{1}$ from 
the expressions.

$$
\begin{aligned}
\frac{\mathcal{Y}_{0}}{\cos \vartheta_{0}} & =\frac{\mathcal{Y}_{1}}{\cos \vartheta_{1}} \\
\frac{n_{0} \mathcal{Y}}{\cos \vartheta_{0}} & =\frac{n_{1} \mathcal{Y}}{\cos \vartheta_{1}} \\
\cos \vartheta_{1} & =\frac{n_{1} \cos \vartheta_{0}}{n_{0}} \\
n_{0} \sin \vartheta_{0} & =n_{1} \sin \vartheta_{1} \\
\sin \vartheta_{1} & =\frac{n_{0} \sin \vartheta_{0}}{n_{1}}
\end{aligned}
$$

Now that we have two independent equations, we can eliminate $\vartheta_{1}$ using trigonometric identities, as follows:

$$
\begin{aligned}
\sin ^{2} \vartheta_{0}+\cos ^{2} \vartheta_{0} & =1 \\
& =\sin ^{2} \vartheta_{1}+\cos ^{2} \vartheta_{1} \\
& =\left(\frac{n_{0} \sin \vartheta_{0}}{n_{1}}\right)^{2}+\left(\frac{n_{1} \cos \vartheta_{0}}{n_{0}}\right)^{2} \\
\sin ^{2} \vartheta_{0}-\frac{n_{0}^{2}}{n_{1}^{2}} \sin ^{2} \vartheta_{0} & =\frac{n_{1}^{2}}{n_{0}^{2}} \cos ^{2} \vartheta_{0}-\cos ^{2} \vartheta_{0} \\
\left(\frac{n_{1}^{2}-n_{0}^{2}}{n_{1}^{2}}\right) \sin ^{2} \vartheta_{0} & =\left(\frac{n_{1}^{2}-n_{0}^{2}}{n_{0}^{2}}\right) \cos ^{2} \vartheta_{0} \\
\frac{\sin ^{2} \vartheta_{0}}{\cos ^{2} \vartheta_{0}} & =\frac{\left(n_{1}^{2}-n_{0}^{2}\right) n_{1}^{2}}{n_{0}^{2}\left(n_{1}^{2}-n_{0}^{2}\right)} \\
\left(\frac{\sin \vartheta_{0}}{\cos \vartheta_{0}}\right)^{2} & =\left(\frac{n_{1}}{n_{0}}\right)^{2} \\
\tan \vartheta_{0} & =\frac{n_{1}}{n_{0}} \\
\vartheta_{0} & =\tan ^{-1}\left(\frac{n_{1}}{n_{0}}\right) \\
\therefore \vartheta_{B} & =\tan ^{-1}\left(\frac{n_{1}}{n_{0}}\right)
\end{aligned}
$$




\section{Appendix B}

\section{MATLAB Simulations}

As a result of fabricating the original proof-of-concept waveguides in-house, certain approximations were made due to limited resources and time constraints that necessitated deviations away from our ideal designs. One such alteration was the use of the Thorlabs LJ1878L2-A cylindrical lens adhered to the surface of a glass slab substrate, as opposed to the use of a single piece of glass that incorporated a quarter-cylinder. As a result, the entrance aperture of the waveguide needed to be widened slightly to allow a sufficient amount of light to couple into the waveguide at the broader angles, where fluence was anticipated to be greatest. The MATLAB simulations were performed to determine the optimum thickness for this allowance, which was eventually determined to be $1.0 \mathrm{~mm}$ wider than the radius of the lens. Waveguides were fabricated with $6.0 \mathrm{~mm}$ entrance apertures as a result of the simulation curves generated by the code included in this appendix.

The code incorporates a variety of necessary aspects that contribute to the coupling efficiency of the cylindrical lens. One such aspect was the fact that as the incident angle changed from 0 to $75^{\circ}$, the beam spot size would grow along its narrowest dimension. The beam was assumed to be an elliptical gaussian, and was simulated using a 3-dimensional intensity distribution, which was projected onto a the waveguide surface at incident angles in $0.2^{\circ}$ increments throughout the full range of anticipated incident angles. 
Another such consideration was that portions of the light would not be coupled into the waveguide, but would instead simply reflect off the silvered rear surface of the glass. Additionally, at broader angles, portions of the projected ellipse would not be coupled into the waveguide as they would be incident beyond the entrance aperture and would consequently reflect of the near face of the waveguide. The code incorporates loops to simulate entrance apertures from 5.0 to $9.0 \mathrm{~mm}$ in increments of $0.5 \mathrm{~mm}$, depicting coupling efficiency resultant from the use of the cylindrical lens. The optimum entrance aperture was chosen as the first curve that demonstrated a maximum at $45^{\circ}$ incidence.

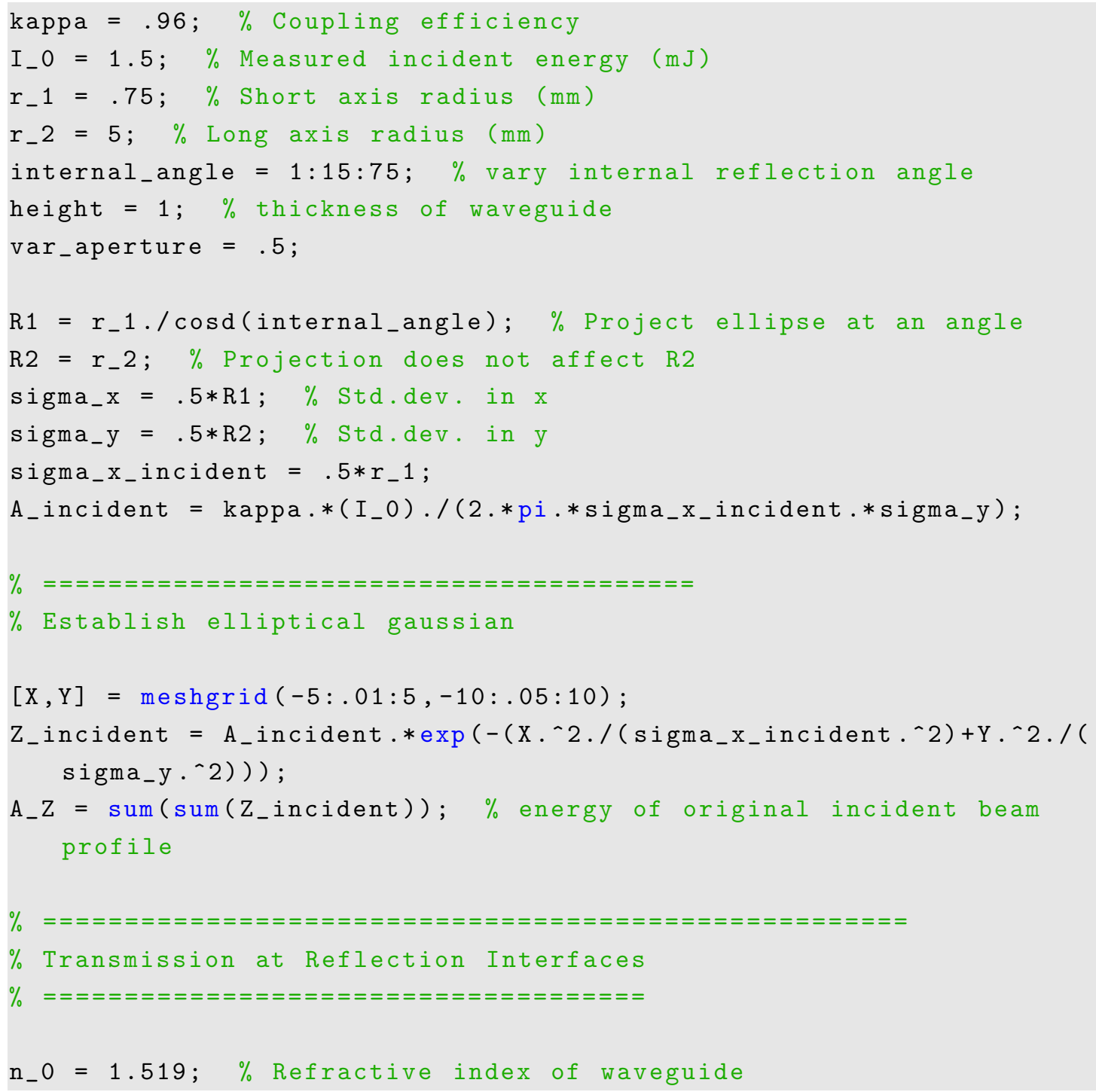




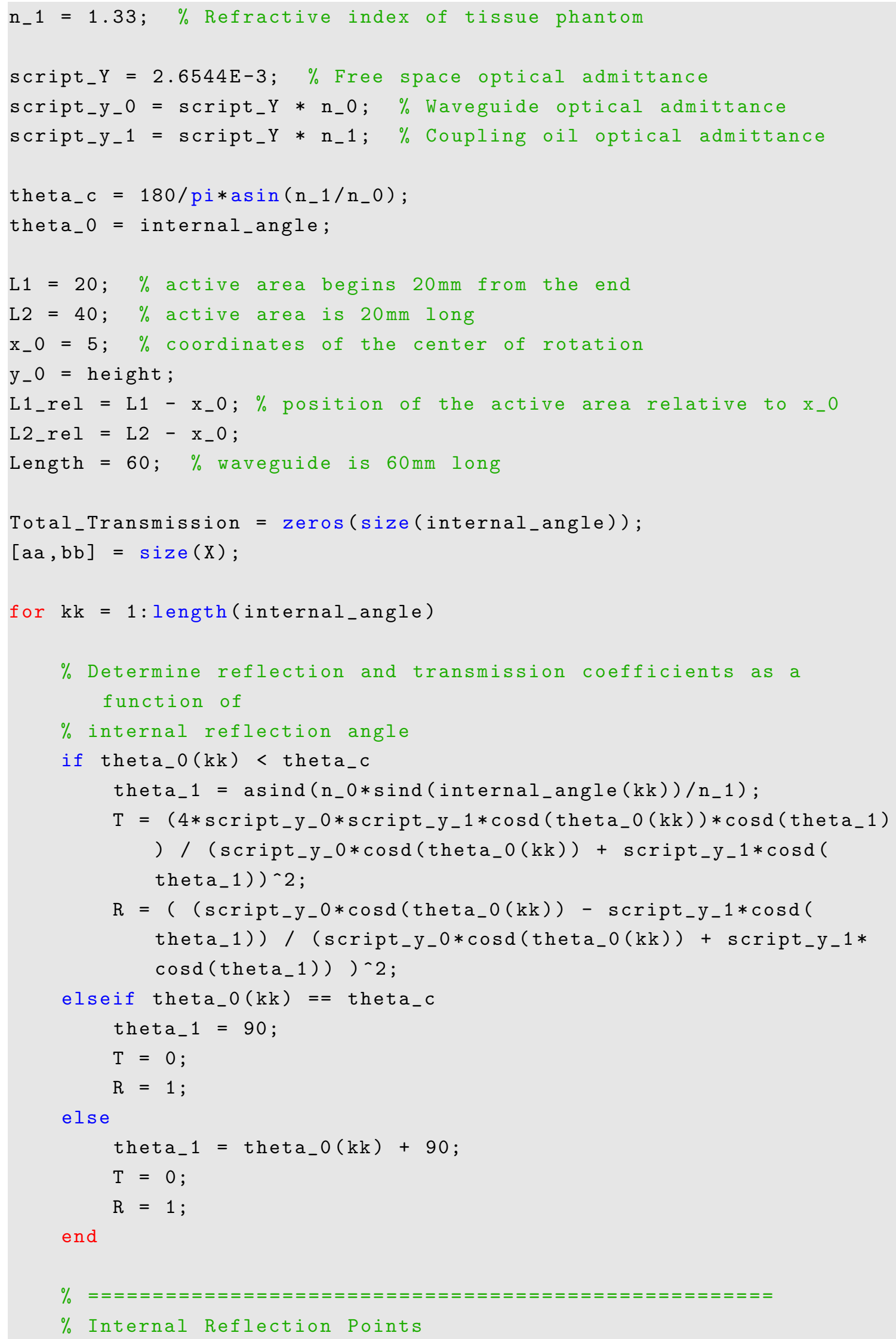




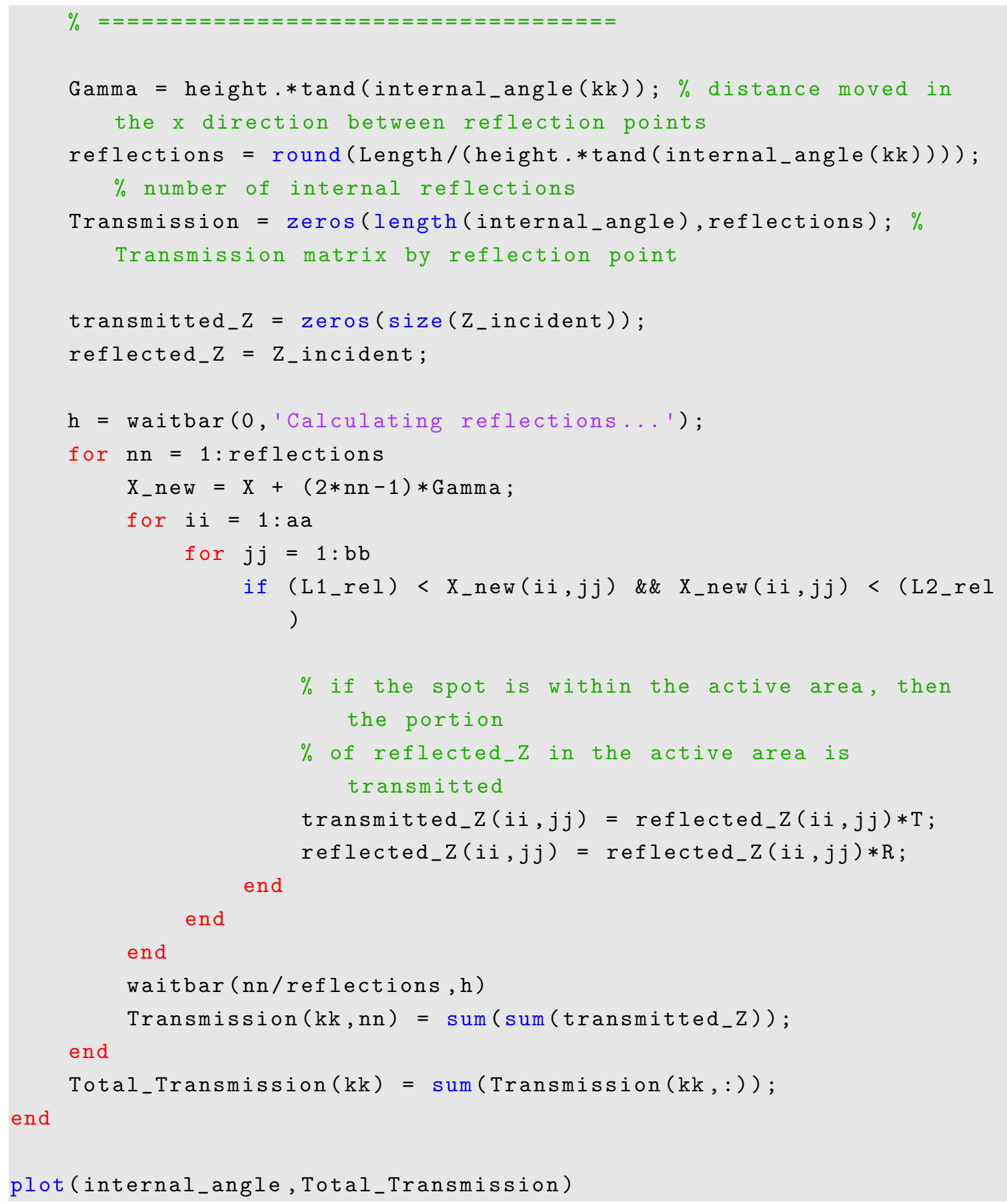




\section{Appendix C}

\section{Error Analysis of Labview-acquired data}

In recording the transmission of laser light through samples of tissue, whether for ultrasonic modulation experiments or otherwise for waveguide active area transmission testing, substantial error was present in the data recorded by LabVIEW. Prior to error analysis, it was not uncommon for the $90 \%$ confidence intervals of any one test to overlap the means of other tests. These overlaps effectively invalidated many conclusions that might have been drawn from the data, which gave rise to the need for appropriate analysis.

This section details the error analysis and compensation process, which narrowed the margin of error for each data set by iteratively accounting for each source of non-random error and thereby reduced the standard deviations of each test individually allowing for the drawing of meaningful inferences. The error analysis was performed in Matlab, rather than in LabVIEW, and the code is included at the end of this section. 


\section{C.1 Identifying sources of error}

The first step in compensating for error is to identify as many independent sources as possible and subsequently categorize them as either random or systematic errors. Random error is herein considered to be the result of unpredictable fluctuations in the measurement equipment, which can be influenced by the quality of the equipment, the accuracy of electronic components, their behavior at different temperatures and energies, and other such physical influences that collectively culminate in the generation of "white noise" in the recorded data. By contrast, systematic error is herein considered to be the result of specific changes in the state of the measurement equipment over time, which may be gradual over time or vary from one data point to the next. For example, during the experiments conducted for Section 4, variations in output laser energy from one shot to the next directly resulted in the recording of peaks and valleys by both the laser energy meter and the integrating sphere photodiode at corresponding data points (i.e. distinct spikes were observed by both measurement devices at corresponding data points with identical indices).

The categorization of error in this manner dictates the method by which the analysis may proceed. The principal distinguishing factor between the two varieties of error is that random error may be averaged to yield a relatively reliable mean with standard deviation predominantly dependent upon the quality of the measurement equipment; whereas statistical analysis of data that is subject to systematic error will yield means that are skewed in the direction of the error source and standard deviations that are almost invariably greater than expected. Given that the random 
error is directly associated with the limitations of the equipment, which are considered to be predominantly dependent on available grant funding, the procedural analysis of systematic error is therefore aimed at removing the non-random deviations such that only the random measurement error remains.

With regard to the waveguide transmission and ultrasonic modulation testing sequences, two hundred pulses were recorded for each test, and two independent measurements were recorded for each pulse. It was strongly suspected that the laser energy was varying significantly from one pulse to the next, which would result in a systematic error that would also be present in both measurements. As such, the error at any given point can reasonably be described for the Laser Energy measurement, $L(x)$, and the Photocurrent, $P(x)$ as:

$$
\begin{aligned}
& L(x)=L_{\text {mean }} \pm \operatorname{Err}_{L-\text { measurement }}(x) \pm \operatorname{Err}_{\text {pulse }}(x) \\
& P(x)=P_{\text {mean }} \pm \operatorname{Err}_{P-\text { measurement }}(x) \pm \operatorname{Err}_{\text {pulse }}(x)
\end{aligned}
$$

wherein each point is defined as a deviation from the respective mean based on the errors that are present. In this representation, $\operatorname{Err}_{L-m e a s u r e m e n t}$ and $\operatorname{Err}_{P-\text { measurement }}$ represent the random measurement error in each measurement device, which are considered distinct from one another. The error in incident pulse energy, Err $r_{p u l s e}$, is present in both data sets and is considered to be resultant from the same source of error. Accordingly it is possible to utilize one data set to remove that specific error from the main data set of interest. It should be noted that doing so will also necessarily merge the random error of both data sets; however, for the purposes 
of these experiments this is considered to be an acceptable concession, because the random error to be removed was an order of magnitude smaller than the combined systematic error.

$$
\begin{aligned}
N_{L E}(x) & =\left(\frac{L(x)-\mu_{L}}{s_{L}}\right) \\
N_{P}(x) & =\left(\frac{P(x)-\mu_{P}}{s_{P}}\right)
\end{aligned}
$$

In order to confirm this assertion, the two data sets were normalized according to Equations C.3 and C.4, where $\mu$ is the mean of the respective test sequence (not the entire data set) and $s$ is the corresponding standard deviation. The normalization function converts the data into a series of points deviating around a mean of zero. If the deviation were entirely random, there would be no observable pattern between the two normalized data sets; however, if the deviation is not entirely random, but instead incorporates a common systematic error, then there would be a strong correlation between the two normalized data sets. Relatively quick options for assessing the presence of systematic error include the Matlab command $r=\operatorname{corr}(X, Y)$, which can be used to determine the correlation between two data sets $\mathrm{X}$ and $\mathrm{Y}$, to simply overlay the two data sets to visually observe the correlation in the data, or otherwise to subtract one normalized data set from the other and determine if the resulting data is normally distributed around a mean of zero with a smaller standard deviation than either of the data presented individually. For example, figure C.1 displays the normalized photocurrent overlaid on the normalized laser energy from a control test sequence for the ultrasound modulation experiments. The figure clearly depicts the 


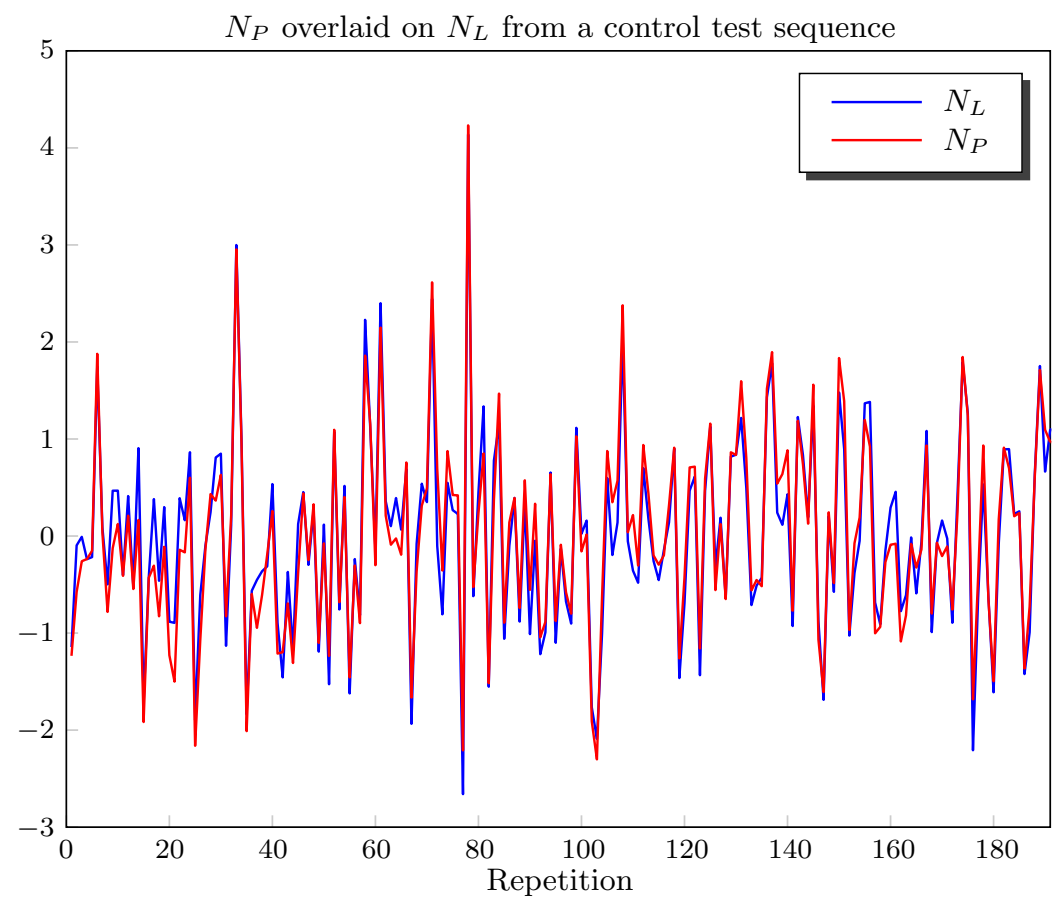

Figure C.1 Overlaying the normalized photocurrent on top of the normalized laser energy measurements reveals strong correlation between the errors of each measurement, which confirms the influence of pulse energy variation on the measured photocurrent.

strong correlation between the error shared by both data sets, with spikes and valleys occurring in $N_{P}$ corresponding with spikes and valleys in $N_{L}$ at the same indices. For further confirmation, this data resulted in a correlation coefficient of $r=0.9700$, which indicates strong correlation between the two and confirms the presence of the suspected systematic error.

However, subtracting the normalized data sets may also reveal other sources of error. For example, Figure C.1 depicts the difference of the two data sets, which reveals that the baseline of one data set appears to "wander" and highlights outliers that have greater than expected deviation from the mean. The analysis in this case revealed that every 12 th data point $( \pm 1)$ of the photocurrent yielded a greater value 
than was expected, as distinct peaks occurred at regular in the normalized difference plot. Each of these peaks occurred beyond the $95 \%$ confidence interval for this data set and were consistently greater than the mean (i.e. there were no peaks that were significantly less than the mean). For the purposes of error analysis, such peaks were programmatically identified as outliers and the corresponding data was removed from both the laser energy and photocurrent data.

\section{C.2 Error compensation}

The following Matlab code was written for the purpose of analyzing light transmission data for sonoillumination experiments associated with the manuscript entitled "Ultrasonic Modulation of Tissue Optical Properties in Ex Vivo Porcine Skin to Improve Transmitted Transdermal Laser Intensity", published in Lasers in Surgery and Medicine. This code analyzes the user-selected dataset output by Labview as a .csv file and proceeds to trim the data to avoid initialization errors, establish normalized datasets for outlier identification and omission, correct for laser energy variation between pulses, and statistically analyze the resultant data for comparison against the control sample dataset.

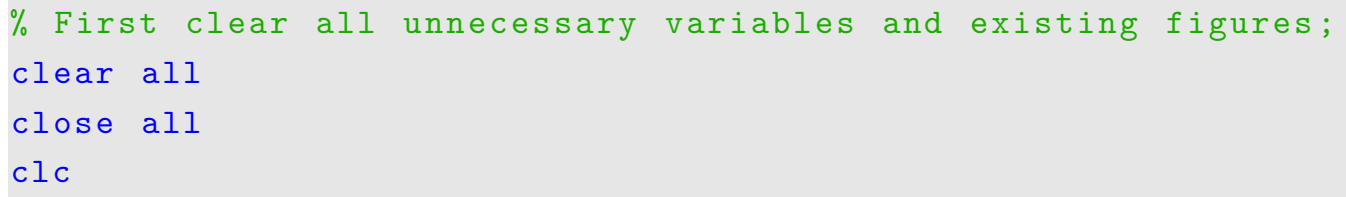

\section{Import data file}

The code to import the data was automatically generated by Matlab using the "import data" option on the main menu bar. This code was saved as a separate function "ImportLabviewCSV_092716.m", which is called below;

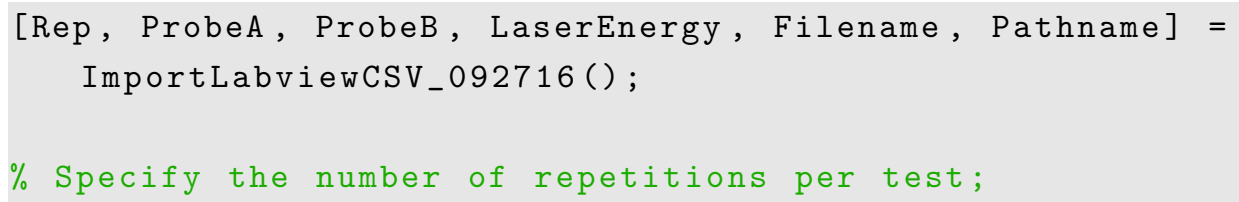


$\mathrm{Num}_{-}$reps $=200$;

Num_tests $=$ floor (length(Rep)./Num_reps);

\section{Trim the data}

Omit 5 data points from the start and the end of each group to avoid skewing the analysis via contamination from potentially saturated detectors, initial laser energy spikes, etc.;

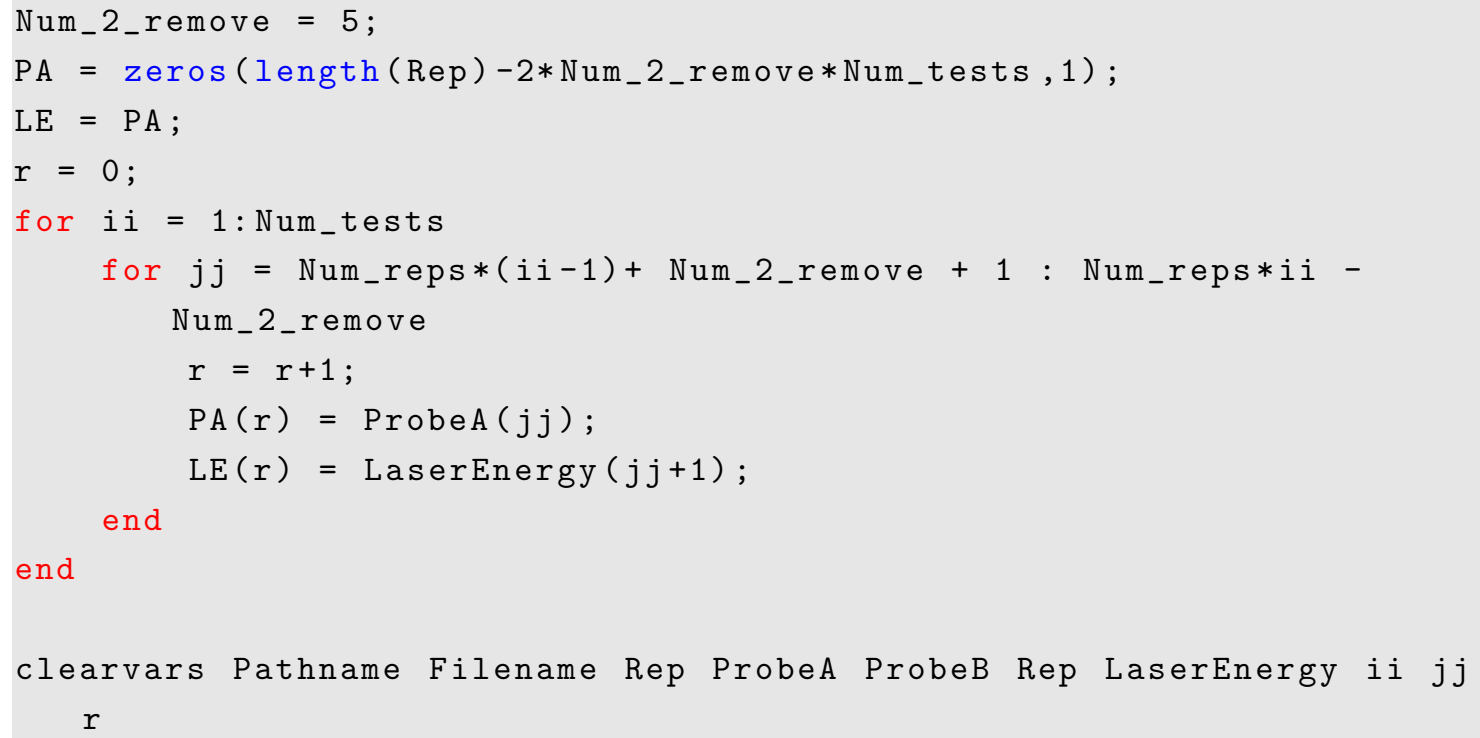

\section{Predefine variables}

Predefine variables to avoid memory allocation issues in subsequent loops;

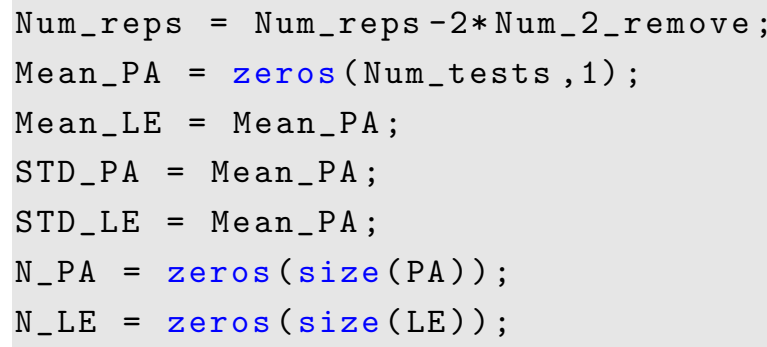

\section{Determine means and standard deviations and build normalized datasets}




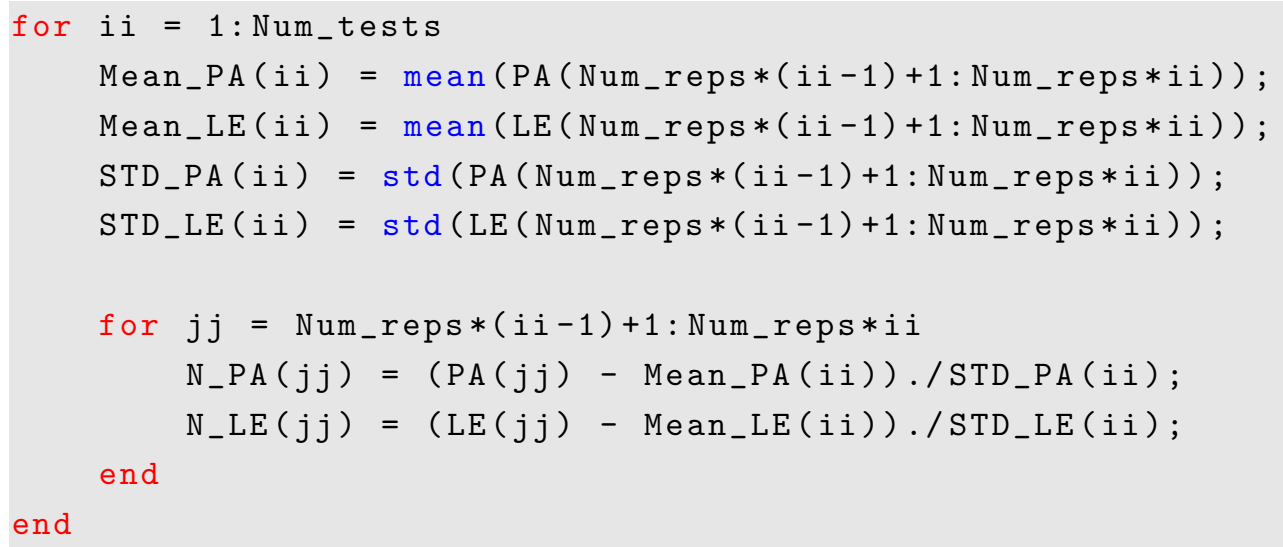

\section{Build N_DIFF dataset}

Establish a dataset that represents the non-random error (N_DIFF), and calculate the respective means and standard deviations. N_DIFF is used in determining the indices of outliers.

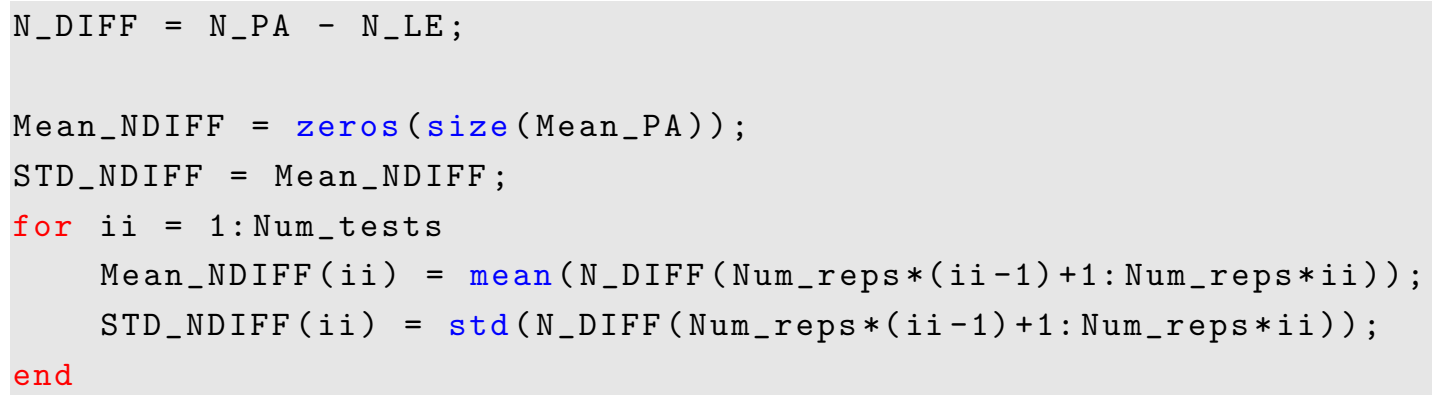

\section{Identify outliers indices}

Utilize N_DIFF to identify data points that occur outside the $90 \%$ Confidence Interval for each group of 190 data points. This was typically fewer than 15 data points, which were later identified as a non-random interference from nearby electronics;

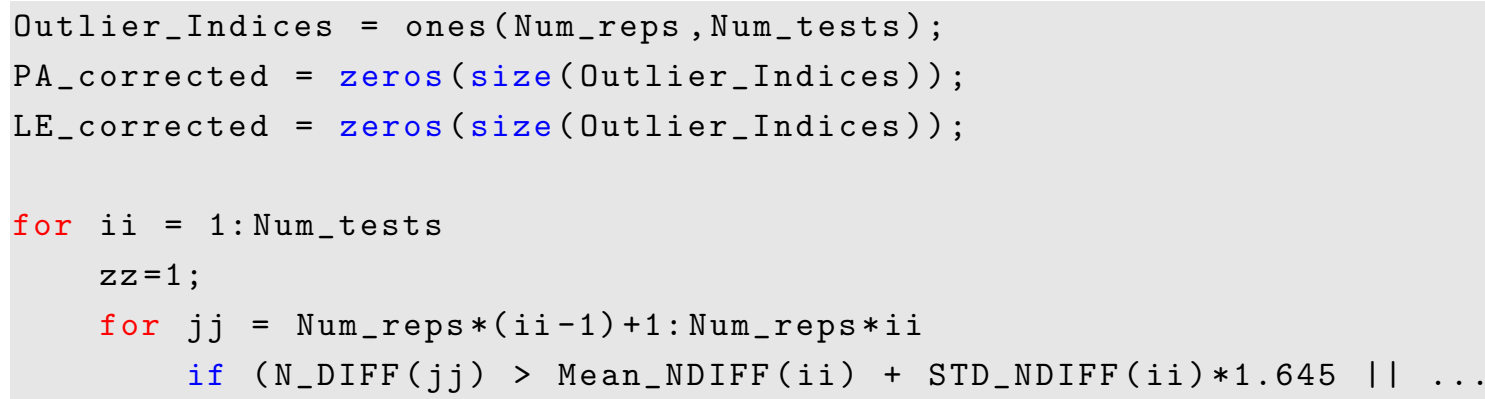




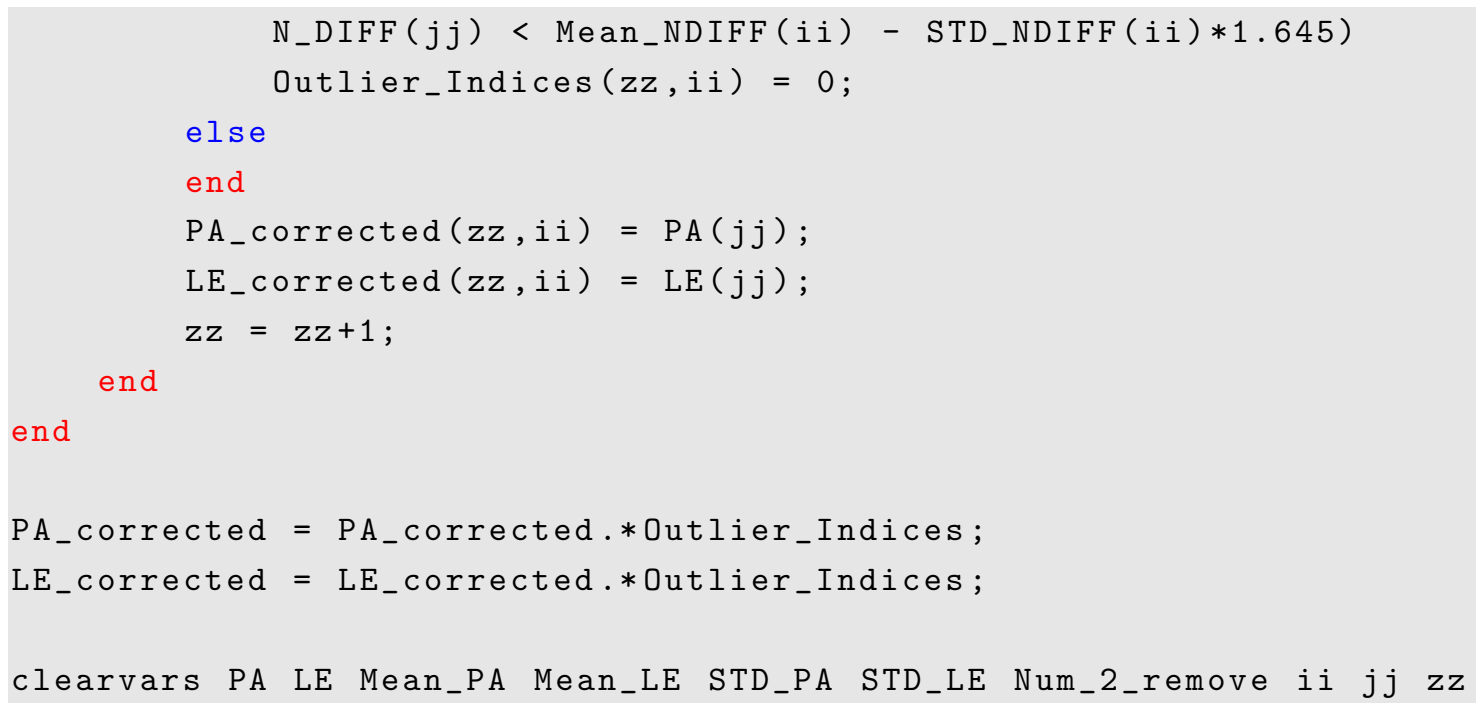

\section{Remove outliers and calculate statistical coefficients}

Establish cell arrays containing individual data sets. Cell arrays are used because outlier removal may result in matrices of different lengths. Calculate means and standard deviations of the corrected data;

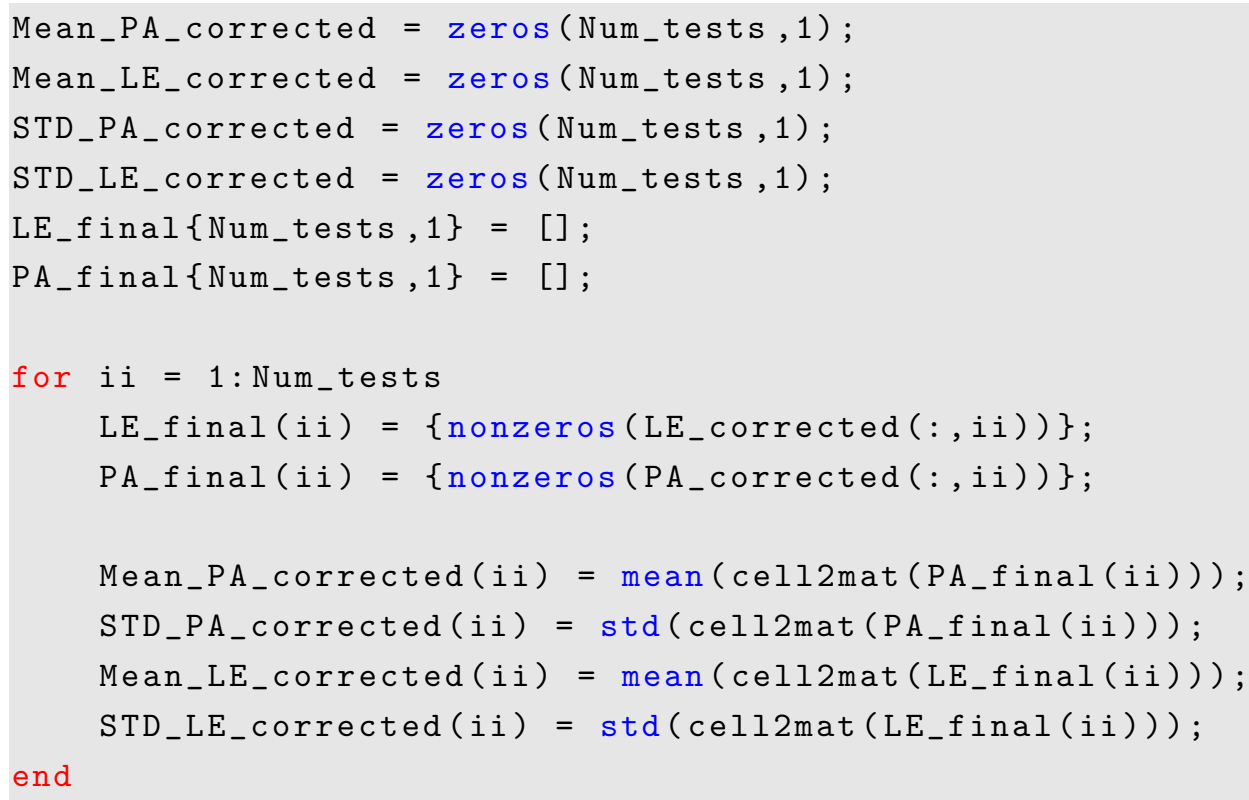

\section{Revise normalized datasets}

Clear previous normalized datasets and re-establish them having omitted the outliers 
identified above. Calculate relevant means and standard deviations;

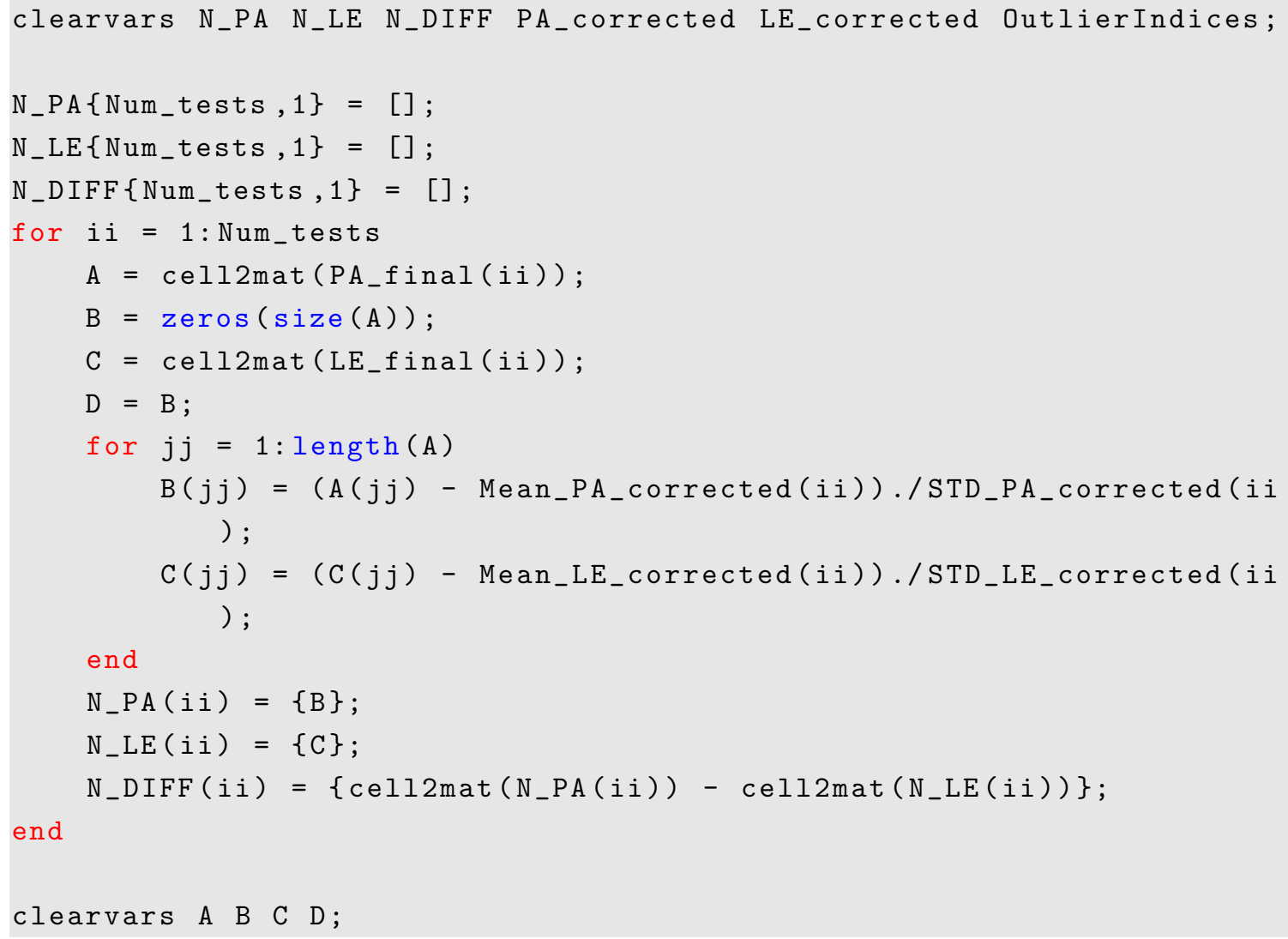

\section{Wandering baseline correction}

Analysis of the data revealed that the baseline recorded by the data acquisition device (Picoscope) was not consistent, but appeared to have a trend that varied with time. This problem did not occur for the laser energy, however. Establish an 11-point wandering baseline from the normalized dataset (common procedure for medical ECG devices), subtract the moving average from the normalized dataset, and then revert back to the original probe voltage data;

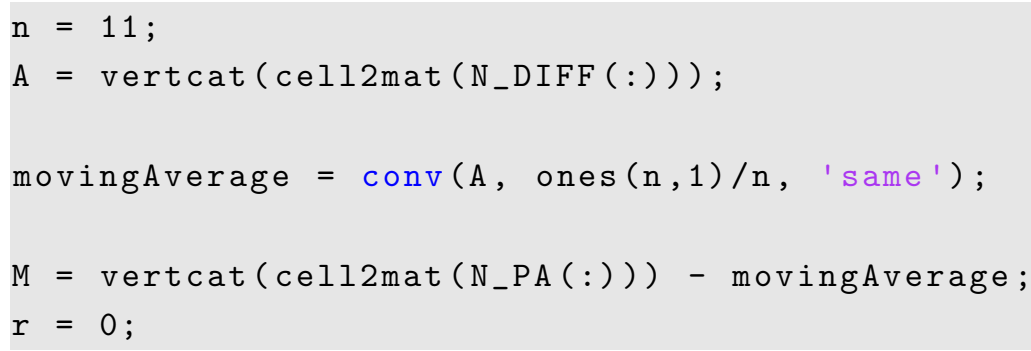




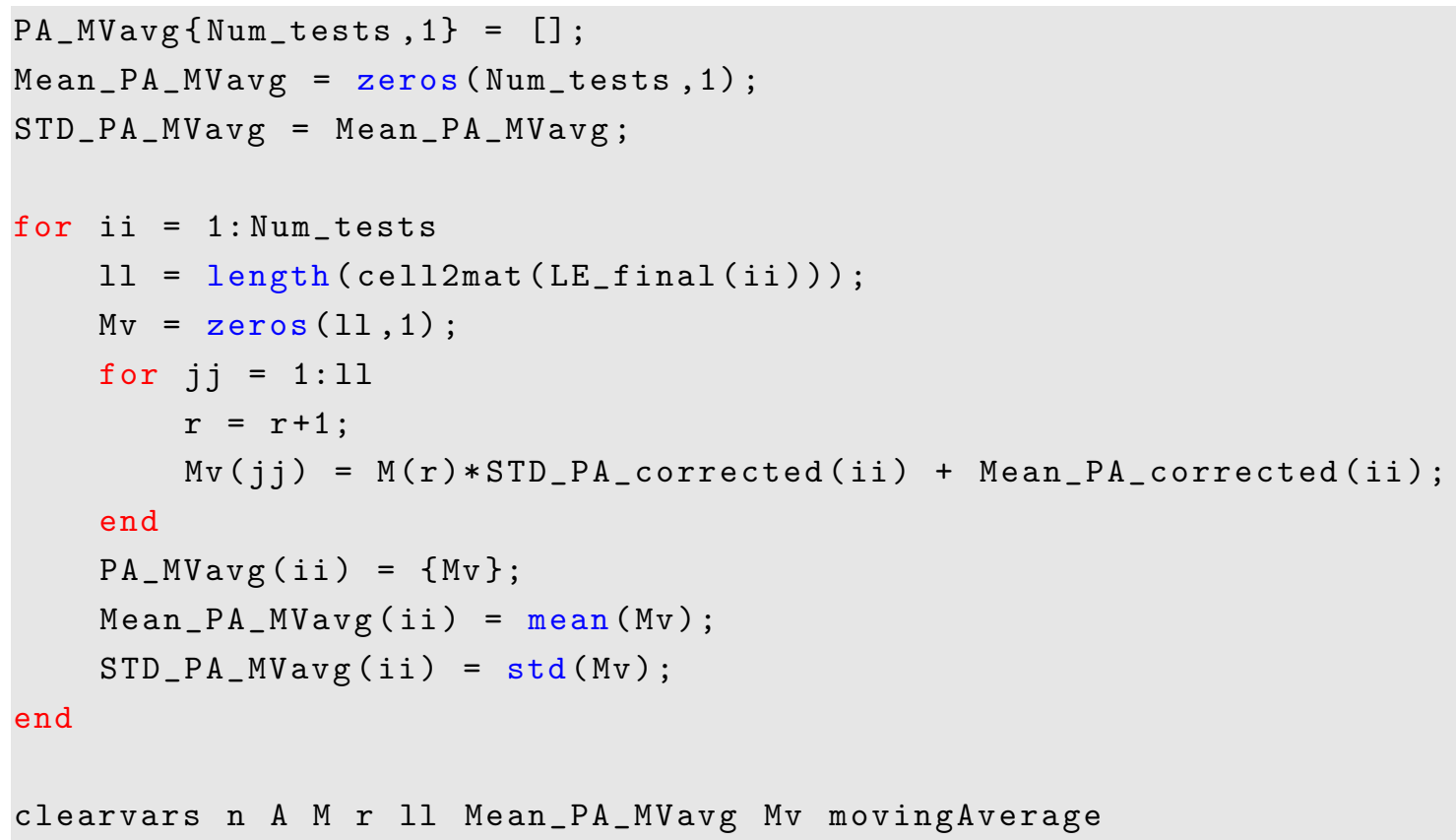

\section{NOTE: Analysis thus far}

At this point, PA_final represents the original data from ProbeA, shortened at the ends, corrected for outliers, and corrected for the wandering baseline. The laser energy variation has yet to be taken into account. Do so by converting probe voltage into optical power and subtracting the difference, which will yield the optical power recorded, corrected for energy variations, with only the white noise of the measurement devices as sources of error.

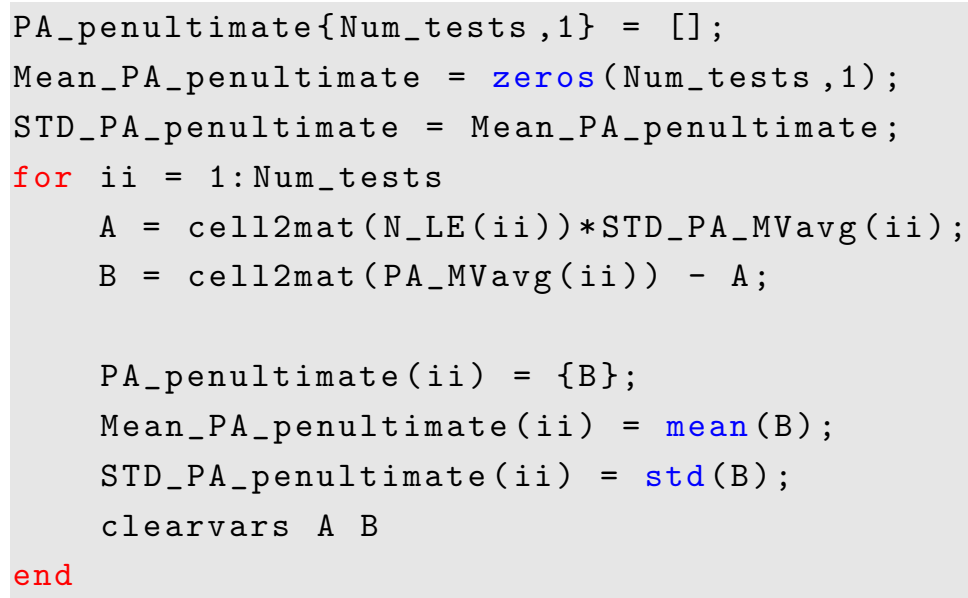




\section{Unit conversion}

Convert the probe voltage to equivalent optical power using a regression equation from the data relating laser energy to probe voltage. The log regression yielded a more accurate fit, so utilize the log relation to convert probe voltage data into equivalent laser energy (optical power).

$\mathrm{y}=>$ Probe Volage

$\mathrm{x}=>$ Laser Energy

BetaFit $=>$ two element matrix of fit coefficients

Linear fit: $\mathrm{y}=\operatorname{BetaFit}(1)^{*} \mathrm{x}+\operatorname{BetaFit}(2) \log$ fit: $\mathrm{y}=\operatorname{BetaFit}(1)^{*} \ln (\mathrm{x})+\operatorname{BetaFit}(2)$

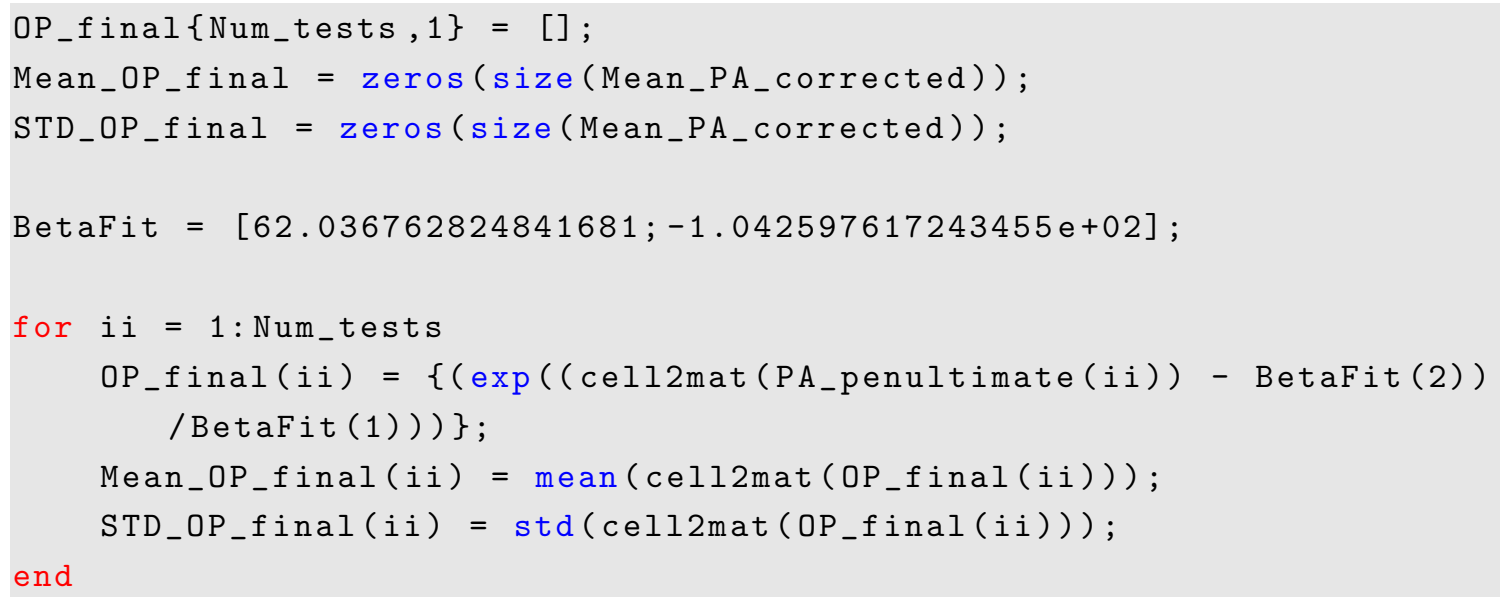

\section{PLOTS}

Figure $1=>$ Scatter plot of all data (post processed) Figure $2=>$ Bar plot of $\%$ difference from control data

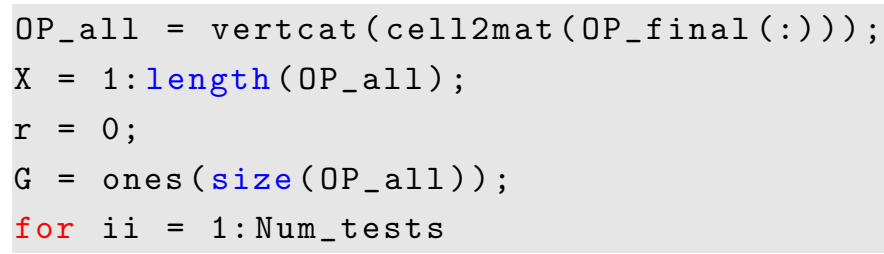




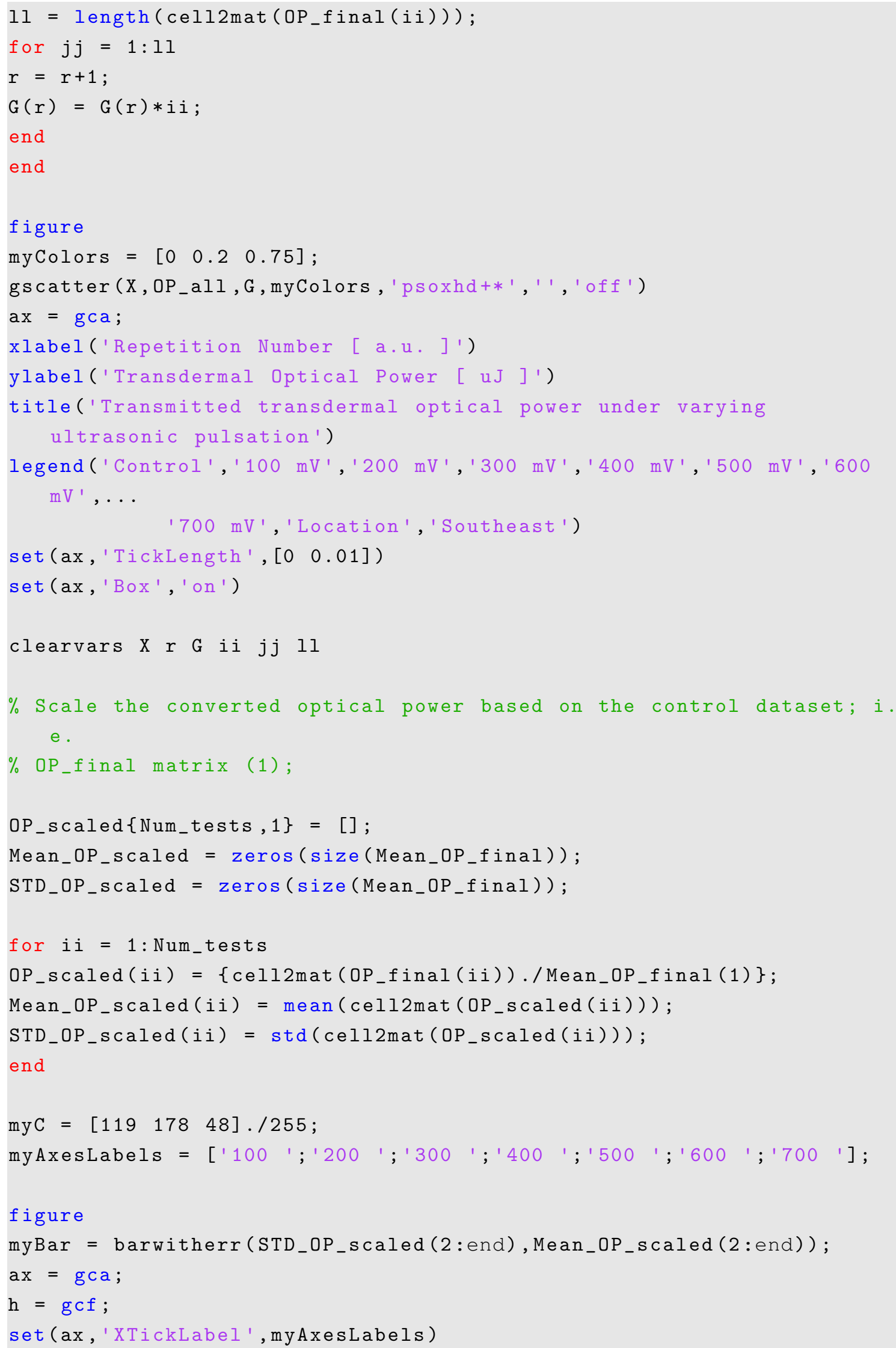




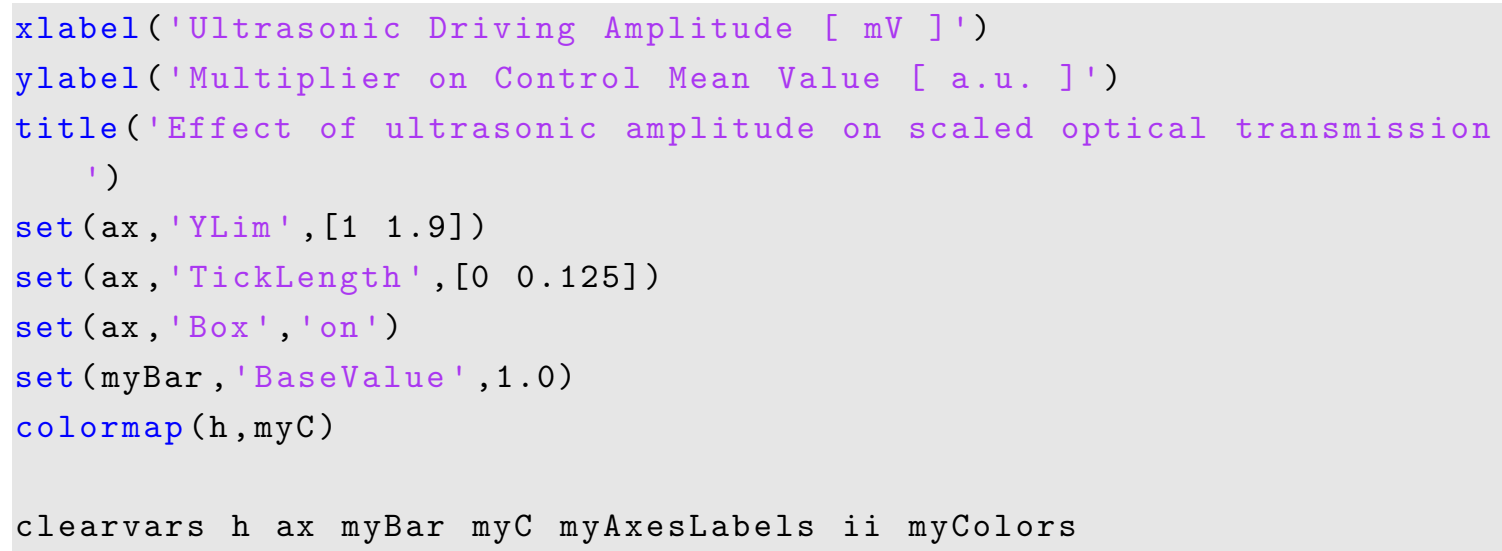




\section{Bibliography}

[1] Paul J.D. Whiteside. Photonic Ablation via Quantum Tunneling. PhD thesis, University of Missouri, May 2015.

[2] Sean W Lanigan. Lasers in dermatology. Medicine, 32(12):21 - 23, 2004.

[3] Sajjad Rajpar and Sean Lanigan. The use of lasers for dermatological conditions. Medicine, 35(8):473 - 476, 2007.

[4] Sean W. Lanigan. Lasers in dermatology. Medical Laser Application, 23(2):51 $-54,2008$.

[5] Roland Kaufmann. Lasers in dermatology - state of the art. Medical Laser Application, 20(2):103 - 109, 2005.

[6] Markolf H. Niemz. Laser-Tissue Interactions: Fundamentals and Applications. Springer, 2004.

[7] Martin J.C. van Gemert and A.J. Welch. Clinical use of laser-tissue interactions. Engineering in Medicine and Biology Magazine, IEEE, 8(4):10-13, Dec 1989. 
[8] Barry E. DiBernardo and Jason N. Pozner. Lasers and Non-Surgical Rejuvenation. Elsevier - Saunders, 2009.

[9] Elizabeth L. Tanzi, Jason R. Lupton, and Tina S. Alster. Lasers in dermatology: Four decades of progress. Journal of the American Academy of Dermatology, 49(1):1 - 34, 2003.

[10] Jean-Paul Ortonne, Amit G. Pandya, Harvey Lui, and Doris Hexsel. Treatment of solar lentigines. Journal of the American Academy of Dermatology, 54(5, Supplement 2):S262 - S271, 2006.

[11] Sonal Choudhary, Mohamed L. Elsaie, Angel Leiva, and Keyvan Nouri. Lasers for tattoo removal: a review. Lasers in Medical Science, 25(5):619-627, 2010.

[12] Michael H. Gold. Lasers and light sources for the removal of unwanted hair. Clinics in Dermatology, 25(5):443 - 453, 2007.

[13] Carsten M. Philipp, Dagmar Scharschmidt, and H. Peter Berlien. Laser treatment of scars and keloids - how we do it. Medical Laser Application, 23(2):79 $-86,2008$.

[14] J.R. Lupton, C.M. Williams, and T.S. Alster. Nonablative laser skin resurfacing using a $1540 \mathrm{~nm}$ erbium glass laser: A clinical and histologic analysis. Dermatological Surgery, 28(9), 2002.

[15] Dong Hun Lee, Yu Sung Choi, Seong Uk Min, Mi Young Yoon, and Dae Hun 
Suh. Comparison of a 585-nm pulsed dye laser and a 1064-nm nd:yag laser for the treatment of acne scars: A randomized split-face clinical study. Journal of the American Academy of Dermatology, 60(5):801 - 807, 2009.

[16] Lisa Carroll and Tatyana R. Humphreys. Laser-tissue interactions. Clinics in Dermatology, 24(1):2 - 7, 2006.

[17] Maurice A. Adatto. Laser tattoo removal: Benefits and caveats. Medical Laser Application, 19(4):175 - 185, 2004.

[18] Akkrapol Mungnirandr, Woraphong Manuskiatti, Pakawadee Hatthanirun, Wanwilai Outtarawichian, Sakda Sookruen, Chaiwat Buathong, and Yongyuth Vajaradul. Laser tattoo removal in thai students. Medical Laser Application, 26(3):126 - 132, 2011.

[19] Katy Burris and Karen Kim. Tattoo removal. Clinics in Dermatology, 25(4): 388 - 392, 2007.

[20] Uwe Wollina and Erich Köstler. Tattoos: surgical removal. Clinics in Dermatology, 25(4):393 - 397, 2007.

[21] Theodora Kossida, Dimitrios Rigopoulos, Andreas Katsambas, and R. Rox Anderson. Optimal tattoo removal in a single laser session based on the method of repeated exposures. Journal of the American Academy of Dermatology, 66 (2):271 - 277, 2012 . 
[22] Eric F. Bernstein. Laser treatment of tattoos. Clinics in Dermatology, 24(1): $43-55,2006$.

[23] Keyvan Nouri and Christopher J. Ballard. Laser therapy for acne. Clinics in Dermatology, 24(1):26 - 32, 2006.

[24] H-J. Laubach, Z. Tannous, R.R. Anderson, and D. Manstein. Skin responses to fractional photothermolysis. Lasers in Surgery and Medicine, 38, 2006.

[25] Pa-Fan Hsiao, Yang-Chih Lin, Cheng-Chieh Huang, and Yu-Hung Wu. Efficacy and safety of a single treatment using a 10,600-nm carbon dioxide fractional laser for mild-to-moderate atrophic acne scars in asian skin. Dermatologica Sinica, 31(2):59-63, 2013.

[26] Karen Riggs, Matthew Keller, and Tatyana R. Humphreys. Ablative laser resurfacing: high-energy pulsed carbon dioxide and erbium:yttrium-aluminumgarnet. Clinics in Dermatology, 25(5):462 - 473, 2007.

[27] Naga B. Meduri. Facial resurfacing: An overview. Operative Techniques in Otolaryngology-Head and Neck Surgery, 18(3):172 - 180, 2007.

[28] Macrene R. Alexiades-Armenakas, Jeffrey S. Dover, and Kenneth A. Arndt. The spectrum of laser skin resurfacing: Nonablative, fractional, and ablative laser resurfacing. Journal of the American Academy of Dermatology, 58(5):719 $-737,2008$. 
[29] Khaled M. Hassan and Anthony V. Benedetto. Facial skin rejuvenation: Ablative laser resurfacing, chemical peels, or photodynamic therapy? facts and controversies. Clinics in Dermatology, 31(6):737 - 740, 2013.

[30] Danielle M. DeHoratius and Jeffrey S. Dover. Nonablative tissue remodeling and photorejuvenation. Clinics in Dermatology, 25(5):474 - 479, 2007.

[31] Albert E. Rivera. Acne scarring: A review and current treatment modalities. Journal of the American Academy of Dermatology, 59(4):659 - 676, 2008.

[32] Zeina Tannous. Fractional resurfacing. Clinics in Dermatology, 25(5):480 - 486, 2007.

[33] Jeffrey S. Orringer, Sewon Kang, Timothy M. Johnson, Darius J. Karimipour, Ted Hamilton, Craig Hammerberg, John J. Voorhees, and Gary J. Fischer. Connective tissue remodeling induced by carbon dioxide laser resurfacing of photodamaged human skin. Archives of Dermatology, 140(11):1326-1332, 2004.

[34] Kenneth C. Nowak, Matthew McCormack, and R. James Koch. The effect of superpulsed carbon dioxide laser energy on keloid and normal dermal fibroblast secretion of growth factors: A serum-free study. Plastic and Reconstructive Surgery, 105(6), 2000.

[35] S. Parker. Verifiable cpd paper: Laser-tissue interaction. British Dental Journal, 202:73-81, 2007. 
[36] A.J. Welch. The thermal response of laser irradiated tissue. Quantum Electronics, IEEE Journal of, 20(12):1471-1481, December 1984.

[37] Ashley J. Welch and Martin J.C. van Gemert, editors. Optical-Thermal Response of Laser-Iradiated Tissue. Lasers, Photonics, and Electro-Optics. Plenum Press, 1st edition, 1995.

[38] Darwin D.-M. Ho, Richard London, George B. Zimmerman, and David A. Young. Laser-tattoo removal - a study of the mechanism and the optimal treatment strategy via computer simulations. Lasers in Surgery and Medicine, 30 (5):389-397, 2002.

[39] Victor E. Ross, George Naseef, Charles Lin, Michael Kelly, Norm Michaud, Thomas J. Flotte, Jill Raythen, and R. Rox Anderson. Comparison of responses of tattoos to picosecond and nanosecond q-switched neodymium:yag lasers. Archives of Dermatology, 134(2):167-171, 1998.

[40] John C. Fisher. Photons, physiatrics, and physicians- a practical guide to understanding interaction of laser light with living tissue- part ii: Basic mechanisms of tissue destruction by laser beams. Journal of Clinical Laser Medicine and Surgery, 11(6):291-303, 1993.

[41] F. Hillenkamp. Laser radiation tissue interaction. Health Physics, 56(5):613-6, May 1989.

[42] Stanley M. Shapshay, editor. Endoscopic Laser Surgery Handbook. Science and 
Practice of Surgery. Marcel Dekker, Inc., 1987.

[43] K. Shokrollahi, E. Raymond, and M.S.C. Murison. Lasers: Principles and surgical applications. The Journal of Surgery, 2(1), 2004.

[44] Robert S. Anderson, Robert E. Hermes, Gennady A. Matyushin, Vladimir S. Nechitailo, and Stephen C. Picarello. Photostability of dye-doped modified polymers at extremely high intensities: Medlite laser systems. Proceedings of the SPIE, 3265:13-20, 1998.

[45] Cynosure medlite c6 medical laser description. 2014. URL http://www. cynosure.com/products/medlite/howitworks.php.

[46] Solta medical fraxel laser system. 2014. URL http://www.solta.com/brands/ fraxel.

[47] L. Goldman, D.J. Blaney, D.J. Kindel, and E.K. Franke. Effect of the laser beam on the skin. Journal of Investigative Dermatology, 40:121-122, 1963.

[48] L. Goldman, D.J. Blaney, D.J. Kindel, D. Richfield, and E.K. Franke. Pathology of the effect of the laser beam on the skin. Nature, 197(912-914), 1963.

[49] Yaniv Barkana and Michael Belkin. Laser eye injuries. Survey of Ophthalmology, 44(6):459-478, 2000.

[50] Daniel F Marcus, Md, and James Ravin. A new report of laser eye injury. Survey of Ophthalmology, 45(3), 2000. 
[51] Michael Belkin. Authors' response: A new report on laser eye injuries. Survey of Ophthalmology, 45(3):262 - 263, 2000 .

[52] Daniele De Luca, Ines Delfino, and Maria Lepore. Laser safety standards and measurements of hazard parameters for medical lasers. International Journal of Optics and Applications, 2(6):80-86, 2012.

[53] Benjamin A. Rockwell, Robert J. Thomas, and Alfred Vogel. Ultrashort laser pulse retinal damage mechanisms and their impact on thresholds. Medical Laser Application, 25(2):84-92, 2010.

[54] Alfred Schirmacher. Eye protection for short and ultra-short pulsed laser systems. Medical Laser Application, 25(2):93 - 98, 2010.

[55] Ming Chen, Chunfei Li, Mai Xu, Weibiao Wang, Shaojie Ma, and Yuxue Xia. Eye-protection glasses against yag laser injury based on the band gap reflection of one-dimensional photonic crystal. Optics and Laser Technology, 39(1):214 $218,2007$.

[56] Moshe Lapidoth, Gal Shafirstein, Dan Ben Amitai, Emmilia Hodak, Milton Waner, and Michael David. Reticulate erythema following diode laser-assisted hair removal: A new side effect of a common procedure. Journal of the American Academy of Dermatology, 51(5):774 - 777, 2004.

[57] Sean W Lanigan. Incidence of side effects after laser hair removal. Journal of the American Academy of Dermatology, 49(5):882 - 886, 2003. 
[58] Christopher A. Nanni and Tina S. Alster. Laser-assisted hair removal: Side effects of q-switched nd:yag, long-pulsed ruby, and alexandrite lasers. Journal of the American Academy of Dermatology, 41(2):165 - 171, 1999.

[59] Sara Friedman and Jonathan Lippitz. Chemical peels, dermabrasion, and laser therapy. Disease-a-Month, 55(4):223 - 235, 2009.

[60] E. Z. Zhang, J. G. Laufer, R. B. Pedley, and P. C. Beard. In vivo high-resolution 3d photoacoustic imaging of superficial vascular anatomy. Physics in Medicine and Biology, 54(4):1035-1046, 2009.

[61] James A. Guggenheim, Thomas J. Allen, Andrew Plumb, Edward Z. Zhang, Manuel Rodriguez-Justo, Shonit Punwani, and Paul C. Beard. Photoacoustic imaging of human lymph nodes with endogenous lipid and hemoglobin contrast. Journal of Biomedical Optics, 20(5), 2015.

[62] E.B. Samson, B.S. Goldschmidt, P.J.D. Whiteside, A.S.M. Sudduth, J.R. Custer, B. Beernsten, and J.A. Viator. Photoacoustic spectroscopy of betahematin. Journal of Optics, 14(6), 2012.

[63] Edward Z. Zhang, Jan Laufer, and Paul Beard. Backward-mode multiwavelength photoacoustic scanner using a planar fabry-perot polymer film ultrasound sensor for high-resolution three-dimensional imaging of biological tissues. Applied Optics, 47(4):561-577, 2008.

[64] B.T. Cox and P.C. Beard. The frequency-dependent directivity of a planar 
fabry-perot polymer film ultrasound sensor. Ultrasonics, Ferroelectrics, and Frequency Control, IEEE Transactions on, 54(2):394-404, 2007.

[65] Paul Morris, Andrew Hurrell, Adam Shaw, Edward Zhang, and Paul Beard. A fabry-perot fiber-optic ultrasonic hydrophone for the simultaneous measurement of temperature and acoustic pressure. The Journal of the Acoustical Society of America, 125(6):3611-3622, 2009.

[66] Paul J. D. Whiteside, Benjamin S. Goldschmidt, Randy Curry, and John A. Viator. Controlled laser delivery into biological tissue via thin-film optical tunneling and refraction. Proceedings of the SPIE, 9303, 2015. doi: 10.1117/12.2078994.

[67] Mason W. Schellenberg, Paul J.D. Whiteside, and Heather K. Hunt. Planar waveguide light transmission modality for backward-mode photoacoustic tomography. Proceedings of the SPIE, 9708, 2016.

[68] Paul J.D. Whiteside, Nicholas J. Golda, Randy D. Curry, and John A. Viator. Regulation of evanescent leaking for contact-based light transmission in biophotonics applications. Applied Optics, 2017.

[69] G. Li, Y. Hashimoto, T. Maruyama, and K. Iiyama. High-efficiency optical coupling to planar photodiode using metal reflector loaded waveguide grating coupler. Optical and Quantum Electronics, 45(7):657-663, 2013.

[70] Milan Milosevic. Internal Reflection and ATR Spectroscopy, volume 176 of Chemical Analysis. Wiley, 2012. 
[71] Siang Ping Ng and J.I. Mackenzie. Planar waveguide laser optimization and characterization employing real-time beam quality measurement. IEEE Journal of Quantum Electronics, 49(2):146-153, Feb 2013.

[72] M.N. Polyanskiy. Refractive index database, 2015. URL: http://refractiveindex.info.

[73] A.D. Rakic, A.B. Djurisic, J.M. Elazar, and M.L. Majewski. Optical properties of metallic films for vertical cavity optoelectronic devices. Applied Optics, 37: 5271-5283, 1998.

[74] B.A. Sexton, B.N. Feltis, and T.J. Davis. Characterisation of gold surface plasmon resonance sensor substrates. Sensors and Actuators A: Physical, 141 (2):471 - 475, 2008.

[75] J.C. Hoogvliet and W.P. van Bennekom. Gold thin-film electrodes: an \{EQCM\} study of the influence of chromium and titanium adhesion layers on the response. Electrochimica Acta, 47(4):599 - 611, 2001. doi: http://dx.doi.org/10. 1016/S0013-4686(01)00793-9.

[76] Paul J.D. Whiteside, Jeffrey A. Chininis, and Heather K. Hunt. Techniques and challenges for characterizing metal thin films with applications in photonics. Coatings, 6(3):35, 2016.

[77] Gabriel Maria Ingo, Giuseppe Guida, Emma Angelini, Gabriella Di Carlo, Alessio Mezzi, and Giuseppina Padeletti. Ancient mercury-based plating meth- 
ods: Combined use of surface analytical techniques for the study of manufacturing process and degradation phenomena. Accounts of Chemical Research, 46 (11):2365-2375, 2013. doi: 10.1021/ar300232e.

[78] Hosuk Lee, Hosun Lee, Jun-Hyuk Park, Han-Ki Kim, Bo Hyun Kong, and Hyung Koun Cho. Optical properties of ultrathin copper thin films sandwiched between nb-doped tio 2 films studied with spectroscopic ellipsometry. Japanese Journal of Applied Physics, 50(5R):055805, 2011.

[79] H. Kuhn and B. A. Wilson. Reflectivity of thin silver films and their use in interferometry. Proceedings of the Physical Society. Section B, 63(10):745, 1950.

[80] E.S.O. New adaptive optics component delivered for the eso vlt, 2 March, 2012. URL http://www.eso.org/public/announcements/ann12015/. URL: http://www.eso.org/public/announcements/ann12015/.

[81] Enrico Savazzi. Digital Photography for Science: Close-Up Photography, Macrophotography, and Photomacrography. Enrico Savazzi, 2011.

[82] Hans Jurgen Karcher. Reflecting Telescope Mechanics. Wiley VCH Cerlag GmbH, 2008.

[83] Jeffrey Chininis, Paul J.D. Whiteside, and Heather K. Hunt. Metal-clad waveguide characterization for contact-based light transmission into tissue. Proceedings of the SPIE, 9689, 2016. 
[84] Ding Lei, Zhang Fanghui, Ma Ying, and Zhang Maili. Aluminum/moo3 anode thin films: an effective anode structure for high-performance flexible organic optoelectronics. Journal of Semiconductors, 33(1), January 2012.

[85] Sihai Chen, Hong Ma, Xinjian Yi, Hongcheng Wang, Xiong Tao, Mingxiang Chen, Xiongwei Li, and Caijun Ke. Optical switch based on vanadium dioxide thin films. Infrared Physics \&3 Technology, 45(4):239 - 242, 2004.

[86] M. Jlassi, I. Sta, M. Hajji, and H. Ezzaouia. Optical and electrical properties of nickel oxide thin films synthesized by sol-gel spin coating. Materials Science in Semiconductor Processing, $21: 7$ - 13, 2014. doi: http://dx.doi.org/10.1016/ j.mssp.2014.01.018.

[87] J. Hotovy, J. Hüpkes, W. Böttler, E. Marins, L. Spiess, T. Kups, V. Smirnov, I. Hotovy, and J. Kováč. Sputtered ito for application in thin-film silicon solar cells: Relationship between structural and electrical properties. Applied Surface Science, 269:81 - 87, 2013. doi: http://dx.doi.org/10.1016/j.apsusc.2012.10.180.

[88] M. Choobtashani and O. Akhavan. Visible light-induced photocatalytic reduction of graphene oxide by tungsten oxide thin films. Applied Surface Science, 276:628 - 634, 2013. doi: http://dx.doi.org/10.1016/j.apsusc.2013.03.144.

[89] K.J. Kuhn. Laser Engineering. Prentice-Hall, 1998.

[90] E. Hecht. Optics. Addison Wesley, 4th edition edition, 2001. 
[91] Max Born and Emil Wolf. Principles of Optics: Electromagnetic theory of propagation, interference and diffraction of light. Cambridge University Press, seventh edition edition, 1999.

[92] Katsunari Okamoto. Fundamentals of Optical Waveguides. Elsevier Academic Press, 2nd edition edition, 2006.

[93] N. J. Harrick. Internal Reflection Spectroscopy. Harrick Scientific Corporation, 3rd edition edition, 1987.

[94] L. Novotny and B. Hecht. Principles of Nano-Optics. Cambridge University Press, 2nd edition, 2012.

[95] S. Zhu, A. W. Yu, D. Hawley, and R. Roy. Frustrated total internal reflection: A demonstration and review. American Journal of Physics, 54(7):601-607, July 1986.

[96] J. Domaradzki, S. Lis, D. Kaczmarek, and S. Patela. Application of spectrophotometry and ellipsometry for determination of optical parameters of optical coating thin films. Students and Young Scientists Workshop, 2010 IEEE International, pages 10-13, 2010.

[97] M. Kusko, M. Purica, and E. Budianu. The optical characterization of thin layers by spectrophotometry measurements. International Semiconductor Conference, 1:90 Vol. 1, 2003. 
[98] Yang Gan. Atomic and subnanometer resolution in ambient conditions by atomic force microscopy. Surface Science Reports, 64(3):99 - 121, 2009. doi: http://dx.doi.org/10.1016/j.surfrep.2008.12.001.

[99] Dimitrios Fotiadis, Simon Scheuring, Shirley A. Müller, Andreas Engel, and Daniel J. Müller. Imaging and manipulation of biological structures with the $\{$ AFM . Micron, 33(4):385 - 397, 2002. doi: http://dx.doi.org/10.1016/ S0968-4328(01)00026-9.

[100] R. Escobar Galindo, R. Gago, A. Lousa, and J.M. Albella. Comparative depthprofiling analysis of nanometer-metal multilayers by ion-probing techniques. TrAC Trends in Analytical Chemistry, 28(4):494-505, 2009.

[101] A. Torres Pérez, A. Hernández Battez, G. García-Atance, J.L. Viesca, R. González, and M. Hadfield. Use of optical profilometry in the $\{$ ASTM $\}$ \{D4172\} standard. Wear, 271(11-12):2963 - 2967, 2011. doi: http://dx.doi. org/10.1016/j.wear.2011.06.016.

[102] S. Sharp, S. Burgess, C. Hodson, and Q. Fang. Thin film properties measurement using sem-based energy dispersive spectroscopy for nanoscience studies. Microscopy and Microanalysis, 14:336-337, 2008.

[103] K.D. Vernon-Parry. Scanning electron microscopy: an introduction. III-Vs Review, 13(4):40 - 44, 2000. doi: http://dx.doi.org/10.1016/S0961-1290(00) 80006-X. 
[104] Jean-Louis Pouchou. X-ray microanalysis of stratified specimens. Analytica Chimica Acta, 283(1):81 - 97, 1993. doi: http://dx.doi.org/10.1016/ 0003-2670(93)85212-3.

[105] M. Procop, M. Radtke, M. Krumrey, K. Hasche, S. Schadlich, and W. Frank. Electron probe microanalysis (epma) measurement of thin-film thickness in the nanometer range electron probe microanalysis (epma) measurement of thin-film thickness in the nanometer range. Analytical and Bioanalytical Chemistry, 374 (631-634), 2002.

[106] Benjamin S. Goldschmidt, Anna M. Rudy, Charissa A. Nowak, Dylan P. Macoubrie, John A. Viator, and Heather K. Hunt. Characterization of $\mathrm{mgf}_{2}$ thin films using optical tunneling photoacoustic spectroscopy. Optics $\&$ Laser Technology, 73:146-155, 2015.

[107] Jorge Pisonero, Beatriz Fernández, Rosario Pereiro, Nerea Bordel, and Alfredo Sanz-Medel. Glow-discharge spectrometry for direct analysis of thin and ultrathin solid films. TrAC Trends in Analytical Chemistry, 25(1):11 - 18, 2006. doi: http://dx.doi.org/10.1016/j.trac.2005.04.019.

[108] Marcus Wilke, Gerd Teichert, Ryota Gemma, Astrid Pundt, Reiner Kirchheim, Henry Romanus, and Peter Schaaf. Glow discharge optical emission spectroscopy for accurate and well resolved analysis of coatings and thin films. Thin Solid Films, 520(5):1660 - 1667, 2011. doi: http://dx.doi.org/10.1016/j. tsf.2011.07.058. 
[109] S. Hosoi, K. Nakajima, M. Suzuki, K. Kimura, Y. Shimizu, S. Fukatsu, K.M. Itoh, M. Uematsu, H. Kageshima, and K. Shiraishi. Observation of si emission during thermal oxidation of $\operatorname{si}\left(\begin{array}{lll}0 & 0 & 1\end{array}\right)$ with high-resolution $\{\mathrm{RBS}\} . \quad$ Nuclear Instruments and Methods in Physics Research Section B: Beam Interactions with Materials and Atoms, 249(1-2):390 - 393, 2006. doi: http://dx.doi.org/ 10.1016/j.nimb.2006.04.036.

[110] R. Nagel, C. Alof, A.G. Balogh, W.M. Arnoldbik, and D.O. Boerma. Study of magnetic multilayers by $\{\mathrm{RBS}\}$ with nanometer resolution. Nuclear Instruments and Methods in Physics Research Section B: Beam Interactions with Materials and Atoms, 183:140 - 145, 2001. doi: http://dx.doi.org/10.1016/ S0168-583X(01)00320-2.

[111] P Bailey, T.C.Q Noakes, C.J Baddeley, S.P Tear, and D.P Woodruff. Monolayer resolution in medium energy ion scattering. Nuclear Instruments and Methods in Physics Research Section B: Beam Interactions with Materials and Atoms, 183:62 - 72, 2001. doi: http://dx.doi.org/10.1016/S0168-583X(01)00384-6.

[112] M. Branescu, C. Naudin, M. Gartner, and G. Nemeş. Depth profiling raman spectroscopy of a thin yba2cu3o7 $\delta$ film. Thin Solid Films, 516(22):8190 - 8194, 2008. doi: http://dx.doi.org/10.1016/j.tsf.2008.04.074.

[113] Yasushi Oshikane, Toshihiko Kataoka, Mitsuru Okuda, Seiji Hara, Haruyuki Inoue, and Motohiro Nakano. Observation of nanostructure by scanning nearfield optical microscope with small sphere probe. Science and Technology of 
Advanced Materials, 8(3):181, 2007.

[114] Bharat Bhushan. Surface roughness analysis and measurement techniques. In Bharat Bhushan, editor, Modern Tribology Handbook, volume 1, chapter 2. CRC Press, 2000.

[115] Michael C. Howland, Alan W. Szmodis, Babak Sanii, and Atul N. Parikh. Characterization of physical properties of supported phospholipid membranes using imaging ellipsometry at optical wavelengths. Biophysical Journal, 92(4): 1306 - 1317, 2007. doi: http://dx.doi.org/10.1529/biophysj.106.097071.

[116] K. Drogowska, Z. Tarnawski, A. Brudnik, E. Kusior, M. Sokołowski, K. Zakrzewska, A. Reszka, N. T. H. Kim-Ngan, and A. G. Balogh. Rbs, xrr and optical reflectivity measurements of ti-tio2 thin films deposited by magnetron sputtering. Materials Research Bulletin, 47(2):296-301, 2012.

[117] E. Chason and T. M. Mayer. Thin film and surface characterization by specular x-ray reflectivity. Critical Reviews in Solid State and Materials Sciences, 22(1): $1-67,1997$.

[118] Jens Als-Nielsen and Des McMorrow. Refraction and reflection from interfaces, pages 69-112. Wiley, 2011. doi: 10.1002/9781119998365.ch3.

[119] A. Kossoy, D. Simakov, S. Olafsson, and K. Leosson. Determining surface coverage of ultra-thin gold films from x-ray reflectivity measurements. Thin Solid Films, 536(0):50-53, 2013. 
[120] Michael F. Toney and Sean Brennan. Measurements of carbon thin films using x-ray reflectivity. Journal of Applied Physics, 66(4):1861-1863, 1989.

[121] Robert W. Collins and Yeon Taik Kim. Ellipsometry for thin-film and surface analysis. Analytical Chemistry, 62(17):887A-890A, 1990.

[122] R. M. A. Azzam and N. M. Bashara. Ellipsometry and polarized light. NorthHolland Pub. Co., 1977.

[123] Karsten Hinrichs, Andreas Furchner, Guoguang Sun, Michael Gensch, Jorg Rappich, and Thomas W. H. Oates. Infrared ellipsometry for improved laterally resolved analysis of thin films. Thin Solid Films, 571(3):648-652, 2014.

[124] I. Kalimanova, N. Ilieva, and M. Georgieva. Ellipsometry and thin films parameters measurement. 28th International Spring Seminar on Electronics Technology: Meeting the Challenges of Electronics Technology Progress, pages 472-475, 2005.

[125] M. Horprathum, J. Kaewkhao, P. Eiamchai, P. Chindaudom, and P. Limsuwan. Investigation of inhomogeneity of $\mathrm{tio}_{2}$ thin films using spectroscopic ellipsometry. Journal of Physics: Conference Series, 417(1), 2013.

[126] Dawn Bonnel, editor. Scanning Probe Microscopy and Spectroscopy: Theory, Techniques, and Applications. Wiley, 2nd edition, 2000.

[127] G. Binnig, C. Quate, and Ch Gerber. Atomic force microscope. Physical Review 
Letters, 56(9):930-933, 1986.

[128] R.F.M. Lobo, M.A. Pereira-da Silva, M. Raposo, R.M. Faria, and O.N. Oliveira Jr. In situ thickness measurements of ultra-thin multilayer polymer films by atomic force microscopy. Nanotechnology, 10:389-393, 1999.

[129] Po-Jui Su and Bernard Haochih Liu. Ion-beam-sputter deposited titanium nitride thin films for conductive atomic force microscope probes. Thin Solid Films, 529(0):317-321, 2013. doi: http://dx.doi.org/10.1016/j.tsf.2012.07.066.

[130] J.I. Goldstein. Scanning Electron Microscopy and X-Ray Microanalysis. Springer, 3rd edition, 2003.

[131] F. Z. Mammeri, L. Chekour, and N. Rouag. Characterization of nitride thin films using sem and edx. Acta Physica Polonica, A, 123(2):294-295, 2013.

[132] Matthew T. Johnson, C. Barry Carter, and Joseph Michael. Sem analysis of oxide thin films and reactions. Journal of the American Ceramic Society, 82 (6):1644-1646, 1999.

[133] Barbara G. Kutchko and Ann G. Kim. Fly ash characterization by sem-eds. Fuel, 85(17-18):2537 - 2544, 2006. doi: http://dx.doi.org/10.1016/j.fuel.2006. 05.016.

[134] Michael C. Meyer, Peter Austin, and Peter Tropper. Quantitative evaluation of mineral grains using automated sem-eds analysis and its application potential 
in optically stimulated luminescence dating. Radiation Measurements, 58:1 11, 2013. doi: http://dx.doi.org/10.1016/j.radmeas.2013.07.004.

[135] E. D. Boyes. Nanoparticle, thin film and surface sensitive analysis in the sem. Microscopy and Microanalysis, 13:584-585, 2007.

[136] B.S. Goldschmidt, A.S.M Sudduth, P.J.D. Whiteside, and J.A. Viator. Total internal reflection photoacoustic spectroscopy. U.S. Patent App. 13/747,172, 2013.

[137] B. S. Goldschmidt, A. S. M. Sudduth, E. Samson, P. J. D. Whiteside, K. Bhattacharyya, and J. A. Viator. Total internal reflection photoacoustic spectroscopy for the detection of beta-hematin. Journal of Biomedical Optics, 17(6), 2012.

[138] Amanda S. M. Sudduth, Benjamin S. Goldschmidt, Edward B. Samson, Paul J. D. Whiteside, and John A. Viator. Total internal reflection photoacoustic detection spectroscopy. Proc. SPIE, 7899, 2011.

[139] Charles Vassallo. Optical Waveguide Concepts, volume 1 of Optical Wave Sciences and Technology. Elsevier, 1991.

[140] H. Angus Macleod. Thin-Film Optical Filters. Institute of Physics, third edition edition, 2001.

[141] Robert G. Hunsperger. Integrated Optics. Springer, 6th edition edition, 2009.

[142] Choon-Gi Choi, Sang-Pil Han, and Myung-Yung Jeong. Two-dimensional poly- 
meric optical waveguides for high-density parallel optical interconnection. $O p$ tics Communications, 235:69 - 73, 2004.

[143] Sir Isaac Newton. Opticks: A Treatise of the Reflections, Refractions, Inflections and Colours of Light. London : Printed for William Innys at the West-End of St. Paul's, 4th edition edition, 1730.

[144] Benjamin S. Goldschmidt. Photoacoustic Evaluation of Surfaces via Pulsed Evanescent Field Interaction. PhD thesis, University of Missouri, May 2014.

[145] I.N. Court and F.K. von Willisen. Frustrated total internal reflection and application of its principle to laser cavity design. Applied Optics, 3(6):719-726, June 1964 .

[146] P.K. Tien and R. Ulrich. Theory of prism-film coupler and thin-film light guides. Journal of the Optical Society of America, 60(10):1325-1337, October 1970.

[147] P.W. Baumeister. Optical tunneling and its applications to optical filters. Applied Optics, 6(5), 1967.

[148] R. Ulrich and R. Torge. Measurement of thin film parameters with a prism coupler. Applied Optics, 12(12), 1973.

[149] Yu Zhu, Chuanjun Yao, Jinbang Chen, and Rihong Zhu. Frustrated total internal reflection evanescent switching. Optics and Laser Technology, 31(8):539 $-542,1999$. 
[150] S.B. Kharchenko, P.M. McGuiggan, and K.B. Migler. Flow induced coating of fluoropolymer additives: Development of frustrated total internal reflection imaging. Journal of Rheology, 47(1523), 2003.

[151] Nicholas J. Goddard, Kirat Singh, Richard J. Holmes, and Behnam Bastani. Resonant grating sensors using frustrated total-internal reflection. Sensors and Actuators B: Chemical, 51(1-3):131 - 136, 1998.

[152] Frederique de Fornel. Evanescent Waves From Newtonian Optics to Atomic Optics. Springer, 1st edition, 2001.

[153] K. Försterling. Über die messung der optischen konstanten sehr dünner metallschichten. Annalen der Physik, 422(8):745-751, 1937.

[154] J. Stuart Nelson, Thomas E. Milner, Bahman Anvari, B. Samuel Tanenbaum, Sol Kimel, Lars O. Svaasand, and Steven L. Jacques. Dynamic epidermal cooling during pulsed laser treatment of port-wine stain: A new methodology with preliminary clinical evaluation. Archives of Dermatology, 131(6):695-700, 1995.

[155] Paul J.D. Whiteside, Chenxi Qian, Nicholas J. Golda, and Heather K. Hunt. Ultrasonic modulation of tissue optical properties in ex vivo porcine skin to improve transmitted transdermal laser intensity. Lasers in Surgery and Medicine, ?(?):?-?, 2017.

[156] Mona Boord. Laser in dermatology. Clinical Techniques in Small Animal Practice, 21(3):145-149, 2008. 
[157] Franz-Xaver Schmid. Biological Macromolecules: UV-visible Spectrophotometry. Encyclopedia of Life Sciences. Nature Publishing Group, 2001.

[158] Rami Nachabe, Benno H.W. Hendriks, Marjolen van der Voort, Adrien E. Desjardins, and Henricus J.C.M. Sterenborg. Estimation of biological chromophores using diffuse optical spectroscopy: benefit of extending the uv-vis wavelength range to include 1000 to $1600 \mathrm{~nm}$. Biomedical Optics Express, 1(5):1432-1442, 2010.

[159] Paul Beard. Biomedical photoacoustic imaging. Interface Focus, 1(4), 2011.

[160] Scott Prahl. Tabulated molar extinction coefficient for hemoglobin in water. Oregon Medical Laser Center, $1998 . \quad$ URL: http://omlc.org/spectra/hemoglobin/index.html.

[161] Steven L. Jacques, Randolph D. Glickman, and Jon A. Schwartz. Internal absorption coefficient and threshold for pulsed laser disruption of melanosomes isolated from retinal pigment epithelium. Proceedings of the SPIE, 468, May 1996.

[162] A. F. Alexis. Lasers and light-based therapies in ethnic skin: treatment options and recommendations for fitzpatrick skin types $\mathrm{v}$ and vi. British Journal of Dermatology, 169:91-97, 2013.

[163] Valery V. Tuchin. A clear vision for laser diagnostics (review). IEEE Journal of Selected Topics in Quantum Electronics, 13(6):1621-1628, 2007. 
[164] Gracie Vargas, Eric K. Chan, Jennifer K. Barton, H. Grady Rylander, and Ashley J. Welch. Use of an agent to reduce scattering in skin. Lasers in Surgery and Medicine, 24(2133-141), 1999.

[165] Dan Zhu, Kirill V. Larin, Qingming Luo, and Valery V. Tuchin. Recent progress in tissue optical clearing. Laser \& Photonics Reviews, 7(5):732-757, 2013.

[166] Baris E. Polat, William M. Deen, Robert Langer, and Daniel Blankschtein. A physical mechanism to explain the delivery of chemical penetration enhancers into skin during transdermal sonophoresis - insight into the observed synergism. Journal of Controlled Release, 158(2):250-260, 2012.

[167] Baris E. Polat, Douglas Hart, Robert Langer, and Daniel Blankschtein. Ultrasound-mediated transdermal drug delivery: Mechanisms, scope, and emerging trends. Journal of Controlled Release, 152(3):330-348, 2011.

[168] Ahmet Tezel and Samir Mitragotri. Interactions of inertial cavitation bubbles with stratum corneum lipid bilayers during low-frequency sonophoresis. Biophysical Journal, 85(6):3502-3512, 2003.

[169] Jinhee Yoon, Donghee Park, Taeyoon Son, Johngbum Seo, J. Stuart Nelson, and Byungjo Jung. A physical method to enahnce transdermal delivery of a tissue optical clearing agent: Combination of microneedling and sonophoresis. Lasers in Surgery and Medicine, 42:412-417, 2010.

[170] Christopher G. Rylander, Thomas E. Milner, Stephan Baranov, and J. Stu- 
art Nelson. Mechanical tissue optical clearing devices: Enhancement of light penetration in ex-vivo porcine skin and adipose tissue. Lasers in Surgery and Medicine, 40(10):688-694, 2009.

[171] Christopher Drew, Thomas E. Milner, and Christopher G. Rylander. Mechanical tissue optical clearing devices: evaluation of enhanced light penetration in skin using optical coherence tomography. Journal of Biomedical Optics, 14(6), 2009 .

[172] I.V Meglinski, A.N. Bashkatov, E.A. Genina, D.Y. Churmakov, and Valery V. Tuchin. The enhancement of confocal images of tissues at bulk optical immersion. Laser Physics, 13(1):65-69, 2003.

[173] Cuncheng Weng and Jing Zhang. Effects of modulation phase of ultrasoundmodulated light on the ultrasound-modulated optical image in turbid media. Ultrasonics, 52(4):472-474, 2012.

[174] Lihong V. Wang and Hsin i Wu. Biomedical Optics: Principles and Imaging. Wiley, 2007.

[175] Paul J.D. Whiteside, Jeffrey A. Chininis, Mason W. Schellenberg, Chenxi Qian, and Heather K. Hunt. Increased epidermal laser fluence through simultaneous ultrasonic microporation. Proceedings of the SPIE, 9706-49, March 2016.

[176] Haowen Ruan, Melissa L. Mather, and Stephen P. Morgan. Ultrasound modulated optical tomography contrast enhancement with non-linear oscillation of 
microbubbles. Quantitative Imaging in Medicine and Surgery, 5(1), 2015.

[177] E. I. Gordon. A review of acoustooptical deflection and modulation devices. Appl. Opt., 5(10):1629-1639, Oct 1966. doi: 10.1364/AO.5.001629. URL http: //ao.osa.org/abstract.cfm?URI=ao-5-10-1629.

[178] Bahaa E. A. Saleh and Malvin Carl Teich. Fundamentals of Photonics, chapter Acousto-Optics, pages 799 - 831. John Wiley \& Sons, 1991.

[179] D. T. Pierce and R. L. Byer. Experiments on the interaction of light and sound for the advanced laboratory. American Journal of Physics, 41(3):314-325, 1973. doi: $10.1119 / 1.1987217$.

[180] A. V. Zakharov, N. V. Polikarpova, and V. B. Voloshinov. The klein-cook parameter in analyzing acousto-optic interaction in acoustically anisotropic media. Bulletin of the Russian Academy of Sciences: Physics, 78(12):1250 - 1253, 2014.

[181] Alexei V. Zakharov, Vitaly B. Voloshinov, and Erik Blomme. Intermediate and bragg acousto-optic interaction in elastically anisotropic medium. Ultrasonics, $51: 745-751,2011$.

[182] W. Leutz and G. Maret. Ultrasonic modulation of multiply scattered light. Physica B: Condensed Matter, 204(1):14 - 19, 1995. doi: http://dx.doi.org/10. 1016/0921-4526(94)00238-Q.

[183] M. Kempe, M. Larionov, D. Zaslavsky, and A. Z. Genack. Acousto-optic tomog- 
raphy with multiply scattered light. Journal of the Optical Society of America A, 14(5), May 1997.

[184] J. M. Schmitt, S. H. Xiang, and K. M. Yung. Speckle in optical coherence tomography. Journal of Biomedical Optics, 4(1):95 - 105, 1999.

[185] Daniel S. Elson, Rui Li, Christopher Dunsby, Robert Eckersley, and Meng-Xing Tang. Ultrasound-mediated optical tomography: a review of current methods. Interface Focus, 1(4), August 2011.

[186] Florian J. Blonigen, Alex Nieva, Charles A. DiMarzio, Sebastien Manneville, Lei Sui, Gopi Maguluri, Todd W. Murray, and Ronald A. Roy. Computations of the acoustically induced phase shifts of optical paths in acoustophotonic imaging with photorefractive-based detection. Applied Optics, 44(18), June 2005.

[187] Byounghwak Lee, Naeho Shin, Kwan Jeong, Myoung-Jin Park, and Byung-Gyu Kim. Nondestructive optical measurement of refractive-index profile of gradedindex lenses. Journal of the Optical Society of Korea, 13(4):468-471, 2009.

[188] Charles A. DiMarzio and Todd W. Murray. Medical imaging techniques combining light and ultrasound. Subsurface Sensing Technologies and Applications, 4(4), October 2003.

[189] Steven L. Jacques. Optical properties of biological tissues: a review. Physics in Medicine and Biology, 58(11), 2013. 
[190] Vasilis Ntziachristos. Going deeper than microscopy: the optical imaging frontier in biology. Nature Methods, 7(8):603 - 614, August 2010.

[191] Bahaa E. A. Saleh and Malvin Carl Teich. Fundamentals of Photonics, chapter Ray Optics, pages 18 - 26. John Wiley \& Sons, 1991.

[192] C. M. Moran, N. L. Bush, and J. C. Bamber. Ultrasonic propagation properties of excised human skin. Ultrasound in Medicine and Biology, 9(1995):1177 1190,21

[193] L. Svilainis and V. Dumbrava. Evaluation of the ultrasonic transducer electrical matching performance. Ultrasound, 62(4):16-21, 2007.

[194] Tan Jian-wen, Liao Rui-jin, Wang Hua, Li Long, and Qiang Sheng-ze. Time domain electrical impedance measurement method for ultrasound transducer. 2011 International Symposium on Bioelectronics and Bioinformatics (ISBB), 2011.

[195] Ricardo Queiros, Pedo Silva Girao, and Antonio Cruz Serra. Single-mode piezoelectric ultrasonic transducer equivalent circuit parameter calculations and optimization using experimental data. IMEKO TC4 Symposium, 2:468-471, 2005.

[196] Kiran Bhattacharyya, Martin Njoroge, Benjamin S. Goldschmidt, Brian Gaffigan, Kyle Rood, and John A. Viator. Detection, isolation, and capture of circulating breast cancer cells with photoacoustic flow cytometry. Proceedings of the SPIE, 8570, 2013. 
[197] Jan Laufer, Clare Elwell, Dave Delpy, and Paul Beard. In vitro measurements of absolute blood oxygen saturation using pulsed near-infrared photoacoustic spectroscopy: accuracy and resolution. Phys. Med. Biol., 50:4409-4428, 2005.

[198] C.M. O'Brien, K. Rood, K.D. Bhattacharyya, T. DeSouza, S. Sengupta, S.K. Gupta, and J.A. Viator. Capture of circulating tumor cells using photoacoustic flowmetry and two phase flow. Journal of Biomedical Optics, 17(6), 2012.

[199] Edward Z. Zhang, Jan Laufer, and Paul Beard. Three dimensional photoacoustic imaging of vascular anatomy in small animals using an optical detection system. Proceedings of SPIE, Photons Plus Ultrasound: Imaging and Sensing, 6437(64370S), 2007.

[200] John G. Webster, editor. The Measurement, Instrumentation, and Sensors Handbook, chapter Medical Imaging, pages 79-13. CRC Press, 1999.

[201] Jerry L. Prince and Jonathan M. Links, editors. Medical Imaging Signals and Systems, chapter Ultrasound Imaging Systems, pages 347 - 371. Pearson Prentice Hall, 2006.

[202] Lihong V. Wang and Junjie Yao. A practical guide to photoacoustic tomography in the life science. Nature Methods, 13(8):627 - 638, 2016.

[203] Tatyana A. Filimonova, Dmitry S. Volkov, Mikhail A. Proskurnin, and Ivan M. Pelivanov. Optoacoustic spectroscopy for real-time monitoring of strongly lightabsorbing solutions in applications to analytical chemistry. Photoacoustics, 1 
(3-4):54-61, 2013.

[204] J.A. Viator, Steven L. Jacques, and S.A. Prahl. Depth profiling of absorbing soft materials using photoacoustic methods. IEEE Journal of Selected Topics in Quantum Electronics, 5(4):989-996, Jul 1999.

[205] J.A. Viator. Characterization of Photoacoustic Sources in Tissue Using Time Domain Measurements. PhD thesis, Oregon Graduate Institute of Science and Technology, August 2000.

[206] Alvin I. Chen, Max L. Balter, Melanie I. Chen, Daniel Gross, Sheikh K. Alam, Timothy J. Maguire, and Martin L. Yarmush. Multilayered tissue mimicking skin and vessel phantoms with tunable mechanical, optical, and acoustic properties. Medical Physics, 43(6):3117 - 3131, June 2016.

[207] Haim Azhari. Basics of Biomedical Ultrasound for Engineers, chapter Appendix A: Typical Acoustic Properties of Tissues. John Wiley \& Sons, 2010.

[208] Alan R. Selfridge. Approximate material properties if isotropic materials. IEEE Transactions on Sonics and Ultrasonics, SU-32(3):381 - 394, May 1985.

[209] Bradley E. Treeby and Ben T. Cox. k-wave: Matlab toolbox for the simulation and reconstruction of photoacoustic wave-fields. Journal of Biomedical Optics, 15(2):021314, 2010.

[210] Alistair P. Rendell Bradley E. Treeby, Jiri Jaros and Ben T. Cox. Modeling 
nonlinear ultrasound propagation in heterogeneous media with power law absorption using a k-space pseudospectral method. Journal of the Acoustic Society of America, 131(6):4324 - 4336, June 2012.

[211] John Gamelin, Andres Aguirre, Anastasios Maurudis, Fei Huang, Diego Castillo, Lihong V. Wang, and Quing Zhu. Curved array photoacoustic tomographic system for small animal imaging. Journal of Biomedical Optics, 13 (2):024007-024007-10, 2008. doi: 10.1117/1.2907157.

[212] Christoph G. A. Hoelen and Frits F. M. de Mul. Image reconstruction for photoacoustic scanning of tissue structures. Applied Optics, 39(31):5872-5883, 2000. 


\section{Vita}

Paul J.D. Whiteside is an award-winning British-American biomedical engineer who earned his B.S., M.S., and Ph.D. at the University of Missouri, in Columbia, MO. He has invented a handful of patentable and patented innovations in response to both laboratory research problems and observed clinical needs, and is an accomplished scientific author, having published a relatively popular Invited Review manuscript in the journal Coatings concerning the characterization of thin films in optical applications, and a research article in the Journal of Optics that was listed amongst their "Highlight Articles" for 2012.

His research career started as an undergraduate working under Dr. Xudong Fan in the area of fiber-based Fabry-Perot cavities for the detection of explosive analytes. Dr. Fan left the University of Missouri for the University of Michigan in 2009, at which point Paul joined Dr. John Viator's research group working on the development of a photoacoustic spectroscopy for determining optical properties of chromophores. He stayed on with Dr. Viator after he finished in B.S. in Biological Engineering in 2010 to pursue a graduate degree developing a photoacoustic tomographic probe for severe burn analysis. In 2013, Dr. Viator accepted a position as Director of the Biomedical Engineering department at Duquesne University in Pittsburgh, PA. This 
complicated Paul's graduate ambitions, which led to Paul merging his M.S. research project with Dr. Randy Curry's research group. This permitted the continuation of his research into selective release waveguides under co-advisement from Drs. Viator and Curry. After concluding his M.S. in 2015, Paul transferred into Dr. Heather Hunt's research group, with whom he had collaborated during his M.S., to ensure that his eventual Ph.D. would be in Bioengineering, since Dr. Curry was a professor in Electrical Engineering. In Dr. Hunt's lab, he served as senior graduate research assistant, which effectively made him the lab manager as well. During his Ph.D., he directed multiple research sub-projects while mentoring undergraduate and graduate researchers in developing technical and professional skills.

There are three principal stages to the development of new technologies: (1) the conducting of basic scientific research, (2) the application of that research through translational research into pragmatic prototypes, and (3) the iterative development of reliably manufactured instruments. Paul's research has been predominantly funded by translational research grants to transition concepts from stage (2) to (3), but distinctly depends upon the basic scientific research conducted by other groups or by his group without an official research grant. Technological innovation depends upon the collective efforts of basic scientists, translational researchers, and development engineers. Undervaluing the contributions of any of these groups undermines the potential for future innovation, and failing to appreciate this fact invariably leads to ruin.

No man is an island. 